Multi-Scale Monitoring and Modelling of the Kapuas River Delta

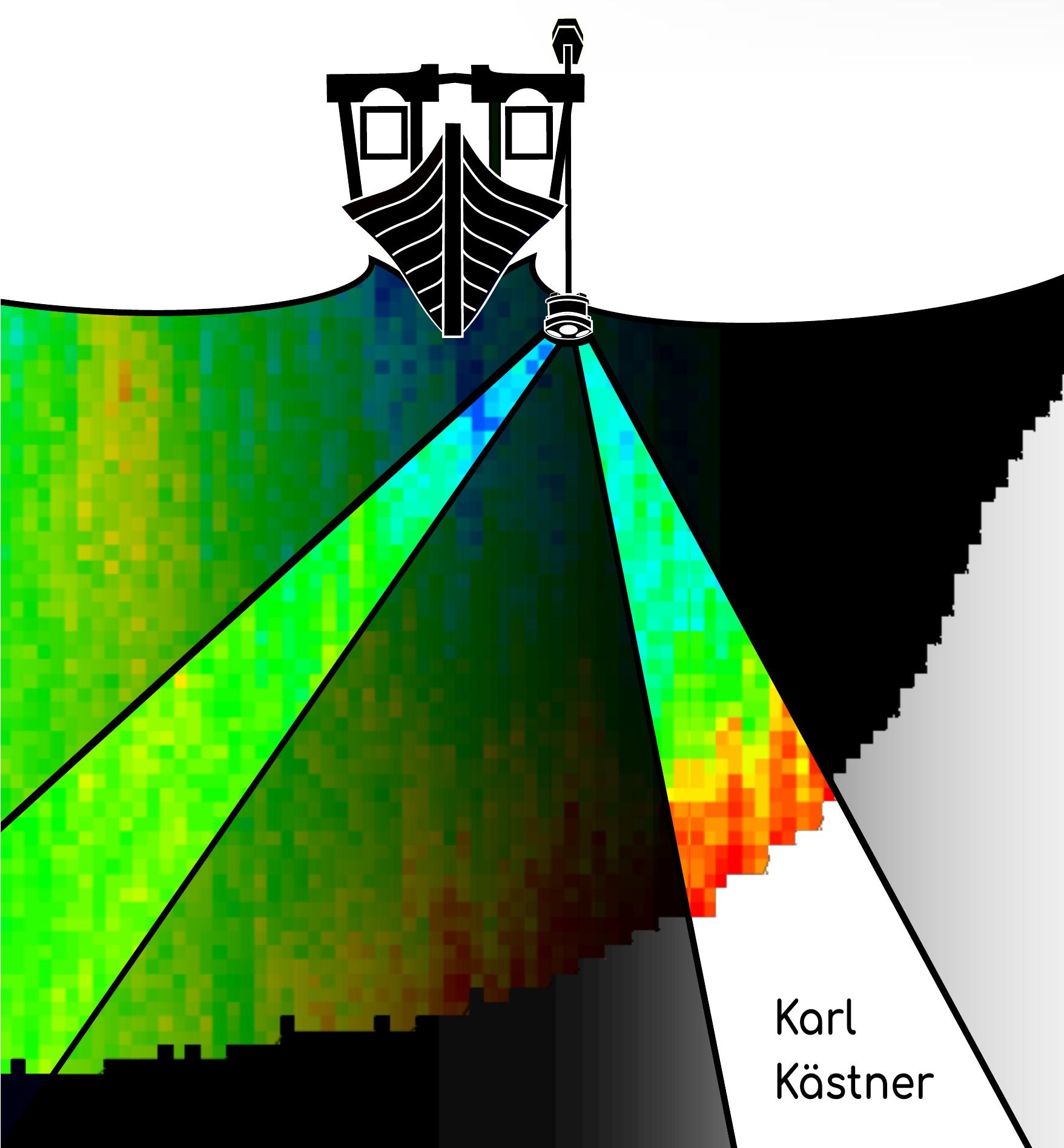




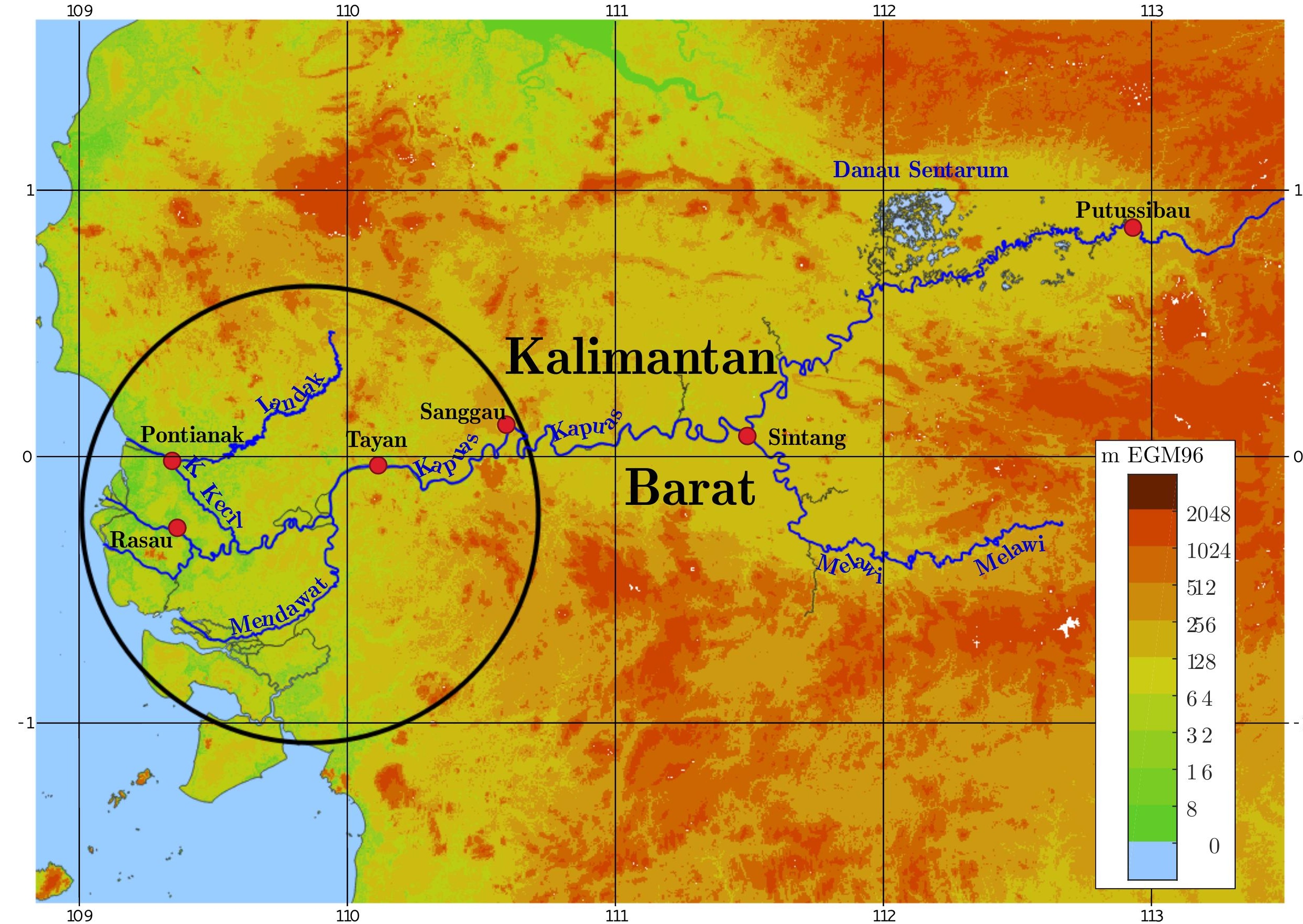




\section{Propositions}

1 Morphodynamics of delta channel networks along the fluvial-tidal transition cannot be understood without considering bank migration. (this thesis)

2 Reach-scale heterogeneity of bed material can strongly influence the morphodynamics on delta-scale.

(Sloff and Mosselman, ESPL, 37(14), 1556-1566, 2012; and this thesis)

3 Sophisticated instruments and methods do not necessarily outperform simple ones.

4 River discharge in the Anthropocene can be measured by the number of plastic bags that float by.

(Jambeck et al., Science, 347(6223), 768-771, 2015)

5 Free software and free information are essential for the success of liberal societies.

(Drahos and Braithwaite, Information Feudalism, Routledge, 2017)

6 Goods and services should be taxed based on the environmental and social impact of their production, use and disposal.

(Johnson et al., Energy Policy, 35(5), 3115-3118, 2007).

Propositions belonging to the thesis titled:

Multi-Scale Monitoring and Modelling of the Kapuas River Delta Karl Kästner

Wageningen, 1 May 2019 



\title{
Multi-Scale Monitoring and \\ Modelling of the Kapuas River Delta
}

\author{
Karl Kästner
}




\section{Thesis committee}

\section{Promotors}

Prof. Dr A. J. F. Hoitink

Personal chair the Hydrology and Quantitative Water Management Group Wageningen University \& Research

Prof. Dr R. Uijlenhoet

Professor of Hydrology and Quantitative Water Management Wageningen University \& Research

\section{Other members}

Prof. Dr P. Passalacqua, The University of Texas, Austin, U.S.A.

Prof. Dr C. Winter, Christian-Albrechts-Universität Kiel, Germany

Prof. Dr T. de Mulder, Ghent University, Belgium

Prof. Dr J. Vilà Guerau de Arellano, Wageningen University \& Research, The Netherlands

This research was conducted under the auspices of the Graduate School for Socio-Economic and Natural Sciences of the Environment (SENSE). 


\section{Multi-Scale Monitoring and \\ Modelling of the Kapuas River Delta}

\section{Karl Kästner}

\section{Thesis}

submitted in fulfilment of the requirements for the degree of doctor at Wageningen University

by the authority of the Rector Magnificus,

$$
\text { Prof. Dr A.P.J. Mol }
$$

in the presence of the

Thesis Committee appointed by the Academic Board

to be defended in public

on Wednesday, 1 May 2019

at 4 p.m. in the Aula. 


\section{K. Kästner}

Multi-Scale Monitoring and Modelling of the Kapuas River Delta vii +217 pages

$\mathrm{PhD}$ thesis, Wageningen University, Wageningen, The Netherlands (2019) With references, with summary in English

ISBN 978-94-6343-411-9

DOI https://doi.org/10.18174/468716 


\section{Abstract}

Rivers in the humid tropics are those with the largest discharges and sediment loads of the world. Their evergreen and everwet catchments are hotspots of biodiversity and their fertile deltas are acres of plenty for the production of rice, palm oil and rubber. At present, tropical rivers, their catchments, and deltas face growing pressure from rapid economic development and climate change, which may permanently deteriorate their ecosystem services. Yet, despite their importance and advancing degradation, relatively little is known about their physiography. This thesis reduces this gap by contributing to our fundamental understanding of tropical rivers.

This thesis in particular addresses fundamental questions regarding the hydro- and morphodynamics of large sand-bedded rivers and their tidally influenced deltas: How can river and tidal discharge be effectively measured? How do the cross-section geometry and bed material change along the fluvialtidal transition? How do these trends differ between the distributaries? How does the tide propagate up-river river? How can sediment transport be efficiently measured with acoustic instruments? How are the discharge and the sediment divided at river bifurcations? To address these questions, the author undertook a year-long journey to West Kalimantan, during which he surveyed and monitored the Kapuas River. The Kapuas River is nearly pristine and thus gives a rare insight into the hydro- and morphodynamics of a river that has not yet been restricted by either dams, dykes or groins. Findings from the survey of the Kapuas River are generalized with idealized models. 



\section{Content}

1 Introduction 1

2 Prerequisites for accurate monitoring of river discharge based on fixed location velocity measurements

3 Distributary channels in the fluvial to tidal transition zone

4 Propagation of tides along a river with a sloping bed

5 Separating the sensed from the unsensed fraction when inferring suspended sediment concentrations from mono-frequency acoustic backscatter

6 Flow and sediment division at two asymmetric bifurcations of a tidally influenced river delta: implications for channel stability 139

7 Role of the inlet width in the deflection of sediment to lateral branches of lowland rivers

8 Synthesis

Summary

Acknowledgements

Bibliography 



\section{Introduction}

\subsection{Motivation}

Rivers and deltas are hotspots of biodiversity (Ward et al., 1999), have been nuclei for the development of human cultures (Maisels, 2003), and remain important places for human settlement (Small and Nicholls, 2003) as well as economic activity. Deltas are used for oil extraction (Ethridge and Wescott, 1984), tidal power generation (Garrett and Cummins, 2005), shipping (Peters, 2001), fishery (Rönnbäck, 1999), agriculture (Sakamoto et al., 2006) as well as recreation (Mopelwa and Blignaut, 2014). Humans extensively influence deltas, locally by changing the land use (Weng, 2002), extracting groundwater and gas (Chaussard et al., 2013), constructing flood defences (Watson and Finkl Jr, 1990), as well as indirectly, by massive dumping of waste (Lebreton et al., 2017), by altering the discharge and sediment supply through the construction of dams (Glenn et al., 1996; Yang et al., 2011) and deforestation (Hamilton, 1987), as well as on a global scale due to climate change induced sea level rise (Nicholls and Cazenave, 2010; Ericson et al., 2006; Hoitink and Jay, 2016). These changes are exaggerated in Asia, due to the high population densities (Yusuf and Francisco, 2009) and rapidly growing economies (Bloom et al., 2000), with Indonesia not being an exception (Chaussard et al. (2013); Budiyono et al. (2015); Murdiyarso et al. (2015); Lebreton et al. (2017)).

The adaptation to and management of these changes consume vast financial resources (Jonkman et al., 2013). This has spurred a recent trend to build with nature and restore delta ecosystems (Day et al., 2007; Cui et al., 2009; Rijke et al., 2012; De Vriend et al., 2015; Brakenridge et al., 2017). But what does a natural delta look like? Which forces shape it? And how does it develop over time? Due to extensive human intervention, most deltas resemble artificial landscapes nowadays, and with progressing development the last pristine deltas will follow. In this thesis, I set out to West Kalimantan, Indonesia, to explore the hydrodynamic and morphological processes that shape the Kapuas 
River Delta, a delta that has not yet been subject to intensive human engineering.

Section 1.2 to 1.7 of this introduction give a general overview of each of the topics addressed in this thesis. Section 1.8 to 1.9.4 introduce the wider research project and an introduction to the Kapuas River. Section 1.10 lists the research questions and goals.

\subsection{Deltas and the fluvial-tidal transition}

Before debouching into the sea, rivers form deltas (Wright, 1978). Different forms of deltas can be distinguished, that are characteristic for the respective relative strengths of river flow, tides and waves (Galloway, 1975). In deltas under the mixed influence of river flow and tides, the flow regime gradually changes from fluvial to tidal. Far upstream from the sea, the flow is uniform, while closer to the sea, it oscillates due to the tide, until the flow finally reverses near the river mouth (Wells, 1995). The channel morphology changes along with the flow: towards the sea, the channel widens, the slope decreases (Parker et al., 2008; Seminara et al., 2012), bedforms become more symmetrical and decrease in magnitude (Berne et al., 1993; Allen, 1974), bed material becomes finer (Frings, 2008), mud drapes appear in the facies (Allen, 1982), meanders become more symmetrical (Marani et al., 2002), the river bifurcates and interconnects with tidal creeks (Wells, 1995) and the salinity increases (Savenije, 1993). Along the way, species of plants and animals in the water and on the banks alter with the conditions (Gingras et al., 2012).

This change along the delta is referred to as the fluvial tidal-transition. This transition is gradual and varies over time with the relative strength of river and tidal flow (Dalrymple et al., 2015). Until recently, not much attention has been paid to the fluvial-tidal transition, which might be due to the circumstance that rivers and estuaries happen to fall into the domains of different academic disciplines of fluvial hydraulics and physical oceanography. Not surprisingly, the transition is shaped by many diverse processes that interact in a complex manner.

The goal of this thesis is to build a bridge between the disciplines in order to achieve a better understanding of the processes along the fluvial-tidal transition. This thesis is structured into four topics: a) methods for measurement and monitoring of discharge and sediment concentration (chapters 2 and 5), b) trends of hydraulic geometry and bed material along the fluvial tidal-transition (chapter 3), c) propagation of tides along the fluvial-tidal transition (chapter 4), d) as well as the division of discharge and sediment at delta-bifurcations 
(chapters 6 and 7). The following paragraphs give a short overview of each of those topics.

\subsection{Discharge monitoring}

Rivers bring life by delivering water and nutrition (Noe and Hupp, 2009), but they also bring catastrophe through extreme flooding (Apel et al., 2004) or falling dry (Smakhtin, 2001). River discharge is an important quantity in the hydrological cycle (Oki and Kanae, 2006) and the only hydrological quantity whose catchment integrated flux can be measured. River discharge is linked to seasonal (Webster and Hoyos, 2004) and decadal variations of the hydrological cycle (Ward et al., 2010), and thus an indicator of the climate (Milly et al., 2005). Rivers are an important source of water for drinking (Vörösmarty et al., 2005), and irrigation (Döll and Siebert, 2002), as well as a sink for the drainage of wastewater (Withers and Jarvie, 2008). As such, rivers also play a role in the waterborne transmission of diseases (Corwin et al., 1995; Gatto et al., 2012). River discharge modulates the intrusion of salinity (Savenije, 1993; Ross et al., 2015) and tides (Godin, 1985). River discharge drives the net transport of sediment (Meade, 1996) and is thus a key factor that influences the morphology of deltas. River tides are linked to delta-morphology (Nienhuis et al., 2018), as well as habitats in the fluvial-tidal transition (Morris et al., 2002; Ensign and Noe, 2018). Since ages, humans have fought with rivers to supply and fend water, but also with each other for access to water (Gleick and Heberger, 2012; Bakker, 2010). Both conflicts are on the rise in a time of growing local and global anthropogenic pressure on river systems (Hirabayashi et al., 2008).

The success of managing water as a resource and as a risk depends on reliable information about its availability. However, many rivers of the world remain ungauged or their data is not openly accessible. River discharge can be predicted with relatively low cost and effort with rainfall-runoff models and satellite measured precipitation (Smith, 1997). However, these methods are not very accurate and require reference discharges for calibration (Beven, 2011; McMillan et al., 2010; Hidayat et al., 2017; Hunger and Döll, 2007). A substantial number of model studies has been published recently, with standard works on rainfall-runoff modelling such as Beven (2011) being cited more than 20 times as frequent than standard works on discharge monitoring such as (Turnipseed and Sauer (2010) and Rantz (1982)). Even though the applications of rainfall-runoff models reach beyond determining discharge, it has to be asserted, there is a lack of knowledge of the ground truth. This hinders 
progress for answering essential questions such as: If and to which extent did rainfall on Borneo change in the recent past? This question has been answered differently by rent model studies, c.f. Kumagai et al. (2013a) and (Takahashi et al., 2017). This is linked to the wider question, how do climate change and deforestation impact precipitation in the humid tropics, and how does this locally affect feed back on the global climate system (Malhi et al., 2008; Snyder, 2010)? There is thus an urgent need for widespread and continuous monitoring of the world's rivers.

The most common method to monitor the discharge of large rivers is to measure the water level and to infer the discharge with a rating curve (Turnipseed and Sauer, 2010). This works well in the intermediate reaches of a river, where there is a good agreement between flow velocity and water level, but less well in gently sloping floodplains and under the influence of tides. For those locations, a direct measurement of the velocity is desirable. The emergence of acoustic Doppler current profilers (ADCPs) has made it is possible to intermittently measure the velocity and thus the river discharge with high accuracy (Gordon, 1989; Simpson and Bland, 2000). The velocity can be continuously measured by rigidly deploying a horizontal ADCP (HADCP) (Le Coz et al., 2008; Vougioukas et al., 2011; Nihei and Kimizu, 2008; Hoitink et al., 2009; Hidayat et al., 2011). However, horizontal ADCPs can only measure the velocity across a small part of a wide river cross-section, as the acoustic signal spreads (Hodges, 2011) and is attenuated (Mellen et al., 1987) with increasing distance from the instrument. The relation between the velocity measured in a small part of the cross-section and the cross-section integrated discharge is complex and sensitive to perturbations. So far, it is not clear under which circumstances the HADCP increases the accuracy of the discharge measurement to an extent that it justifies the higher cost and effort. Chapter 2 of this thesis explores the limits of HADCPs for the monitoring of discharge in large rivers.

\subsection{Hydrodynamics of the fluvial-tidal transition}

The prediction of tides and mean water level is important for navigation (Parker and Huff, 1998), tidal power generation (Brooks, 2011), flood protection (Vellinga et al., 2014), as well as management of salinity intrusion (Savenije, 2012a), and freshwater habitats (Ensign and Noe, 2018; Jay and Kukulka, 2003). Tides at sea represent a stationary harmonic series and can be reliably forecasted based on past observations and knowledge of the perpetual movement of the celestial bodies (Doodson, 1921a; Egbert and Erofeeva, 2002). In contrast, tides in rivers are modulated by non-stationary rainfall-runoff, which compli- 

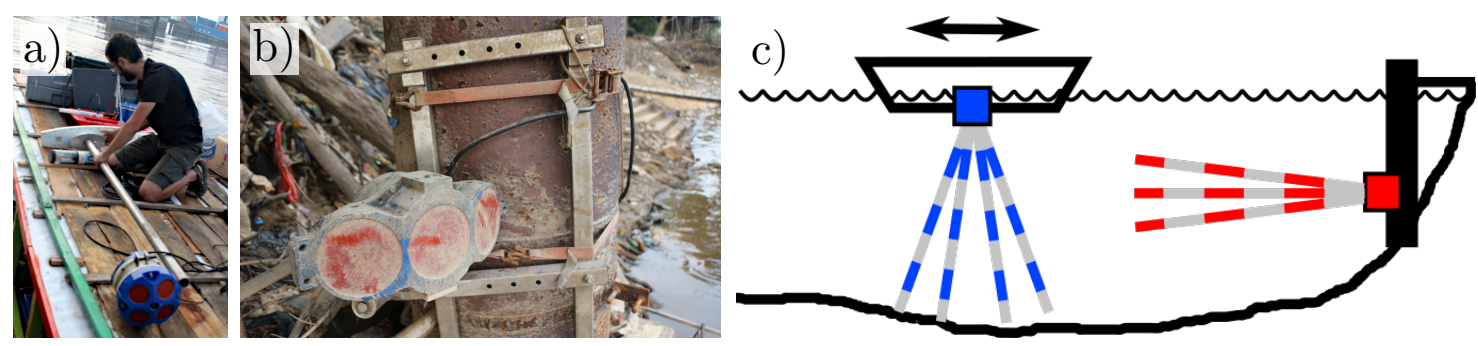

Figure 1.1: a) Vertical ADCP on a pole before being mounted to the side of a moving vessel; b) Horizontal ADCP rigidly mounted to a jetty, submerged during a period of low river flow; c) Schematic deployments of vertical (blue) and horizontal (red) ADCPs

cates their analysis and prediction (Jay and Kukulka, 2003; Jay and Flinchem, 1997; Kukulka and Jay, 2003b).

The flow in rivers and deltas is described by the shallow water equations, which represent the continuity of flow and balance of momentum with external forces (Chow, 1959; Dronkers, 1964; Henderson, 1966). The shallow water equations are weakly non-linear and have in general no explicit solution, but the cross sectionally integrated flow through tidal rivers can be understood by linearizing the equations and considering only the most important terms, i.e. those with the largest magnitude. Gravity drives the flow both in the river and the sea, but the forces that balance the gravity change along the fluvial-tidal transition. Far upstream, the hydrograph of large rivers changes slowly so that the pull of gravity is balanced by friction. Flood waves travel downstream with a velocity that is nearly uniquely defined by the water level. Once the alluvial plain is reached, the height of the flood wave rapidly diffuses out (Ponce and Simons, 1977). Close to the sea, the tide forces the surface elevation and the oscillating pull of gravity is primarily balanced by the oscillation of the flow.

\section{Tidal hydrodynamics}

Although the tide consists of an infinite number of harmonic constituents (Doodson, 1921a), they combine so that the tide appears to the observer as a single wave at any time. As the tide is a long wave, at a particular location, the observer merely perceives it as the slow rising and falling of the water elevation, which recurs twice per day for the semidiurnal tide. This allows one to characterise the tidal wave by only a few parameters: surface elevation amplitude, surface elevation phase, current amplitude and current phase. For practical use, these are usually tabulated as the tidal range, and the time of low and high water as well as the time of slack water. These parameters vary over 
the spring-neap cycle (Kvale, 2006) and longer periods (Ray and Cartwright, 2007), but can be determined from the tidal constituents.

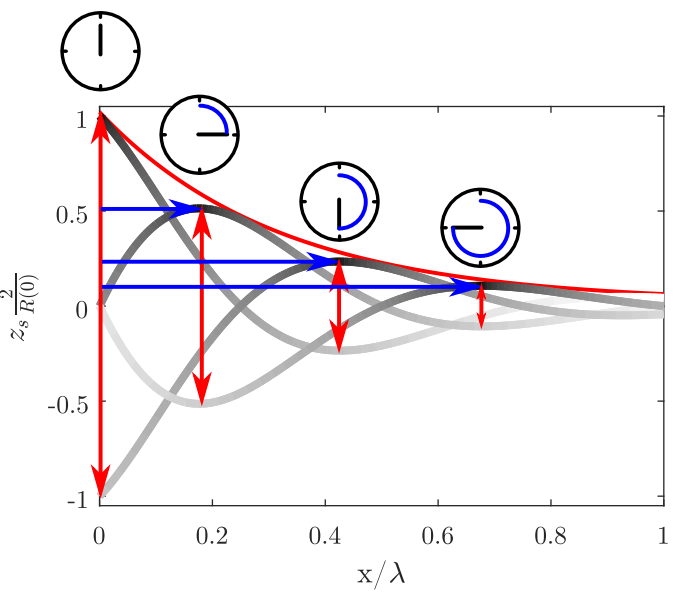

Figure 1.2: Water surface elevation $\left(z_{s}\right)$ as the tidal wave propagates upstream along an idealized tidal-river, red arrows indicate the tidal range $(R)$, blue arrows the phase

How the tide propagates up-river is determined by the cross-section geometry and friction, which are modulated by the river flow. When the friction is low and the crosssectional area convergences (decreases) in the direction of the propagation of the tide, then the tidal range increases according to Green's Law (Jay, 1991; Toffolon et al., 2006). The frictional damping reduces the tidal range exponentially along the river (Ippen, 1966; LeBlond, 1978). Tidal rivers typically resemble long non-converging channels, where friction is stronger than convergence. Rivers thus differ from ideal tidally dominated estuaries, where friction and convergence balance each other. Friction damps the tidal wave so that the tidal range gradually diminishes as the wave travels upstream. Along with the damping, friction also increases the phase difference between the surface elevation and velocity (Savenije and Veling, 2005). River discharge strongly increases the strength of the friction. The increased friction delays high water slack and advances low water slack, up to a point from where no flow reversal occurs farther upstream.

Friction is the strongest non-linearity in shallow water and as such disperses the tidal wave, i.e. it generates additional frequency components. Components with higher frequency than the incoming tide are called overtides. These are typically integer multiples of the dominant tidal frequencies. River discharge thus modulates the overtide spectrum. Without river discharge, the overtide is odd and small in magnitude (Parker, 1991). With strong river discharge, the overtide is even and large in magnitude (Godin, 1999). The even overtide makes the wave crest propagate faster than the trough, it thus sharpens the shape of the tide and increases the tidal asymmetry. Additionally generated components with lower frequency are called subtidal harmonics. Their period is related to the spring-neap cycle and is typically fortnightly or monthly (LeBlond, 1979; Buschman et al., 2009). As lower frequency components are 
less rapidly damped, the amplitude of the subtidal harmonics can exceed that of the main tidal species far upstream. The point where this occurs is considered as the upstream end of the fluvial-tidal transition (Jay et al., 2016).

\section{Analysis and prediction}

The tide consists of an infinite number of constituents of which many are of similar magnitude and frequency. Long time series are required to distinguish constituents of similar frequency. This does not hinder the harmonic analysis of the tide at sea, as the frequency spectrum varies little over time (Pawlowicz et al., 2002; Godin, 1991c). However, river discharge modulates the spectrum of the river tide over much shorter periods of time then what is required to separate important tidal constituents so that it is not meaningful to apply conventional harmonic analysis. To cope with this, constituents of similar frequencies are grouped into species who are then determined and predicted by short-term Fourier transform or wavelet analysis (Jay and Flinchem, 1997; Kukulka and Jay, 2003b). Alternatively, the river discharge can be explicitly considered in non-stationary harmonic analysis (Matte et al., 2014). The analysis of tidal species allows one to estimate the discharge inversely based on tidal records, which has been used to reconstruct historic river discharges (Moftakhari et al., 2016).

The river tide can also be determined by approximating the solution

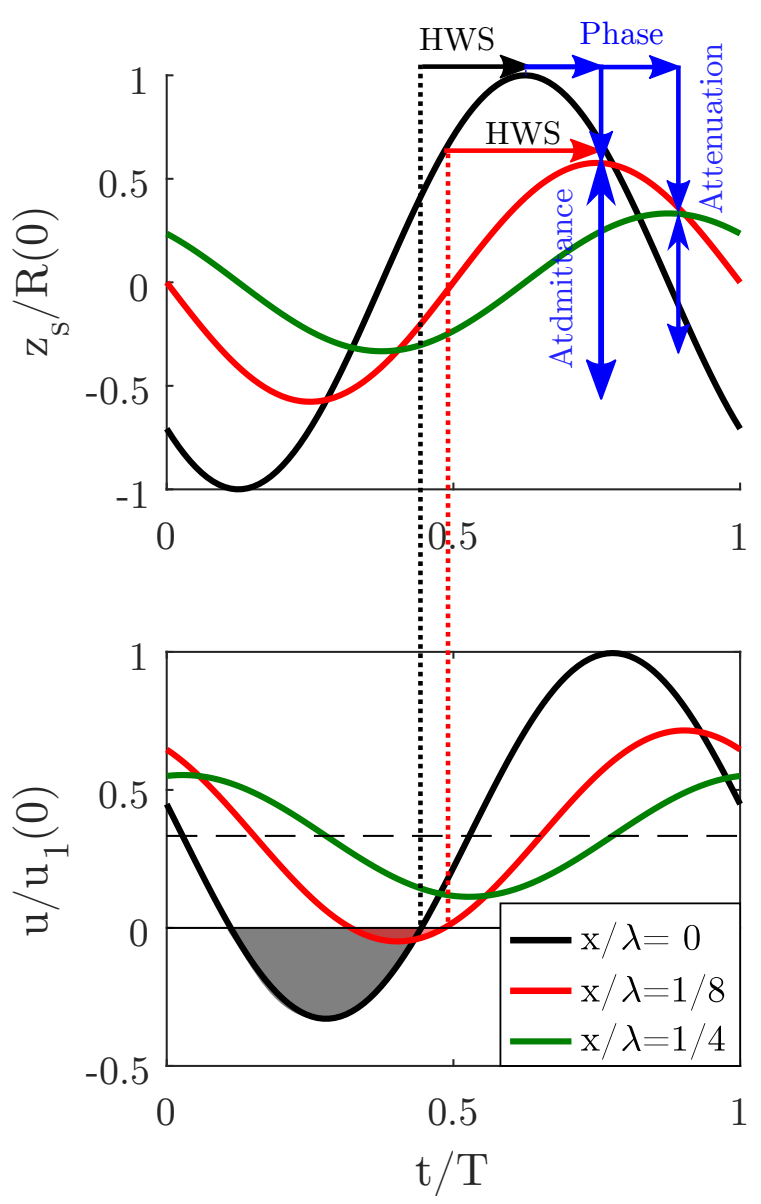

Figure 1.3: Relationship between surface elevation and velocity; as the tide propagates up a river, the velocity amplitude is attenuated at the same rate as the surface elevation amplitude, the time lag high and slack water increases (HWS), the duration of reverse flow decreases (filled areas), and far upstream the flow does not any more reverse. 
to the shallow water equations. One way is to linearise and transform the shallow water equations into the wave equation, which yields a set of ordinary differential equations in one variable for each frequency component (Ippen, 1966; Godin, 1985; Jay, 1991; Alebregtse and de Swart, 2016). Alternatively, the solution can be approximated with the envelope method (Savenije et al., 2008; Cai et al., 2014). Essential for the computation of river tides is the approximation of the non-linear friction term, for which different methods were developed by Lorentz (Terra et al., 2005), Godin (1991a), Dronkers (1964) and Savenije (2001). Although the simplified set of equations helps to understand the propagation of river tides, the general form can only be solved numerically, as the solution can only be expressed in form of analytic functions for trivial cases. This is because the tidally averaged water depth changes along the river depending on the river discharge. The variation of the tidally averaged water depth and its effect on the propagation of the tide has so far received little attention. Cai et al. $(2014,2016)$ considered the drawdown effect for a river with negligible bed slope. In such a case the tidally averaged water surface always forms a drawdown curve, and the tide propagates far upstream (Godin and Martínez, 1994). However, with a sloping bed, the water surface forms a drawdown curve during low flow. The sloping bed also limits the length of tidal intrusion and thus cannot be ignored (Dalrymple et al., 2015). Chapter 4 of this thesis presents a detailed analysis of this phenomenon.

\subsection{Morphology of the fluvial-tidal transition}

As a river flows downstream, it is met by confluences so that its discharge and sediment transport increase (Rodríguez-Iturbe and Valdes, 1979). To accommodate the larger fluxes, the cross-sectional area as well as the flow velocity increase along the way. The cross-section geometry tends to an equilibrium where the sediment transport is balanced with the supply that prevails when the river is near bankfull flow. This is referred to as the concept of formative discharge (Carling, 1988). The relation between cross-section geometry, and formative discharge can be expressed as empirical power laws, which is referred to as the hydraulic geometry (Leopold and Maddock, 1953; Wilkerson and Parker, 2010). These show that along the channel with the discharge, width increases more rapidly than depth and depth increase more rapidly than velocity. The concept of hydraulic geometry can be extended to include slope, roughness and grain size. These three quantities decrease in the downstream direction (Blom et al., 2016). The hydraulic geometry is linked to channel patterns. Wide rivers with high loads of coarse sediment are braiding (Crosato 
and Mosselman, 2009), while rivers with fine suspended load are meandering (Schumm, 1985; Eaton et al., 2010). Rivers are typically graded, i.e. their hydraulic geometry changes exponentially towards the sea, with the exception of the gravel-sand transition, where the size of the bed material decreases rapidly over a short distance (Smith and Ferguson, 1995; Frings and Kleinhans, 2008a). In most large rivers, the gravel-sand transition occurs before the delta plain is reached.

Deltas are, in contrast to rivers, transgressive, as the sediment delivered by the river is deposited at the delta front so that the delta progrades into the sea (Shaw et al., 2016). During extreme flood events, new channels can avulse through the delta plain to bypass a prograded delta lobe (Coleman, 1988; Stouthamer and Berendsen, 2007). The old channel does not necessarily fill in so that several distributaries can coexist at the same time to form a distributary channel network. Sea level change (Jerolmack, 2009) and subsidence (Meckel et al., 2007) lead to constant changes of the delta. The hydraulic geometry and channel pattern of deltas and estuaries also differ from those of rivers. This is because the relative addition of water and sediment by confluences is relatively low, while the sea influences the morphology by backwater dynamics (Lamb et al., 2012; Chatanantavet et al., 2012), storm-surges (Eelkema et al., 2009), and tides (Hoitink and Jay, 2016).

The form of deltas depends mainly on the relative strength of river, tides and waves (Galloway, 1975), but is also influenced by the size of the transported sediment (Orton and Reading, 1993). The characteristic shape of a delta can change along the coast (Sexton and Murday, 1994) and over time, when delta lobes are prograding or recessing (Boyd et al., 1992).

When the river flow dominates the morphodynamics, then prograding delta lobes either form a single channel that is flanked by levees, which is the case when the friction is low so that the river outflow forms a jet (Canestrelli et al., 2014), or the channel recursively bifurcates around mouth bars and forms a fractal pattern, when the friction is high (Edmonds and Slingerland, 2007). Vegetation consolidates mouth bars and contributes to their transition to islands with surrounding channels (Mikhailov, 1966). The cross-sectional areas of these channels decrease towards the sea at each bifurcation (Mikhailov, 1970; Andren, 1994). Short waves generated by the wind inhibit the recursive bifurcation, as they close windward branches and transform the mouth bars into a barrier (Nardin and Fagherazzi, 2012). The front of wind-dominated deltas has a cuspate shape (Nienhuis et al., 2015).

In contrast, tidally influenced distributaries widen towards the sea and do not bifurcate recursively. The widening of the distributaries towards the sea 

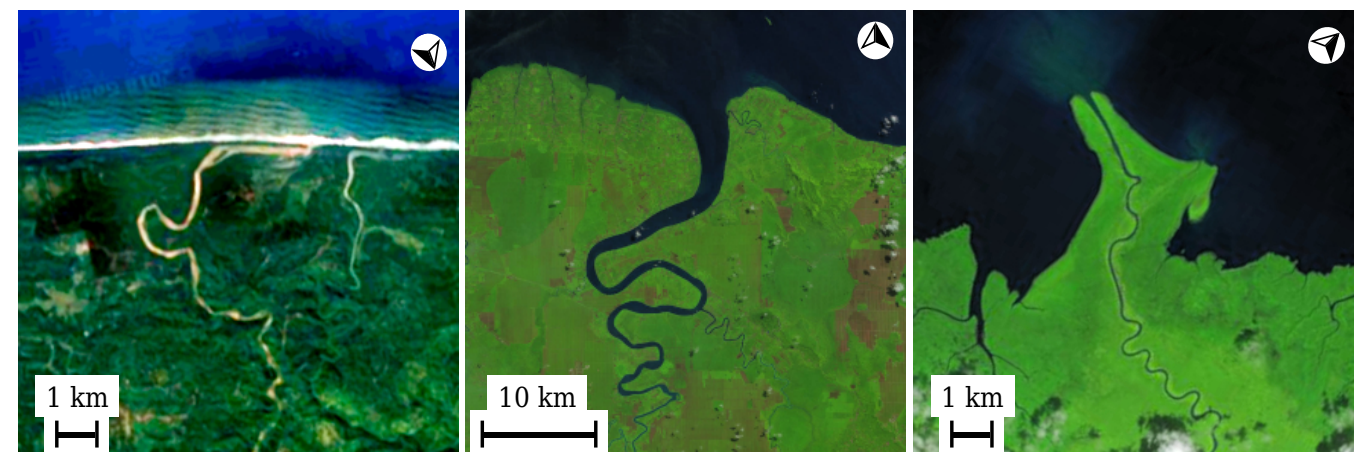

Figure 1.4: Estuaries and deltas with clearly distinguished morphology, left: wave dominated: Tji Wulan on Java Java (Google Maps), centre: tidally dominated: Batang Sadong on Borneo, right: river dominated: Banderi Waidu on Papua (Landsat, courtesy of USGS)

reduces the net flow velocity and sediment transported by the river flow alone. This is balanced by transport through river-tide interaction. The area of tidal inlets scales with the tidal range (D'Alpaos et al., 2009; Nienhuis et al., 2018). The widening is caused by higher flow velocities during reverse flow near the channel banks than near the channel centre. The combination of levees that form around the jet during outflow and erosion of the banks during reverse flow form trifurcations (Fagherazzi et al., 2015). Tides also form creeks that are loosely connected to the fluvial network and do not carry a significant amount of river discharge (Sassi et al., 2012).

The cross-section of tidal funnels typically converges exponentially, as the tidal flow decreases exponentially along the channel (Savenije et al., 2008). River-tide interaction is necessary for the increase of the cross-sectional area towards the sea, but does not suffice on its own to determine the trend of the aspect ratio. Tidally dominated distributaries converge primarily in their width, with relatively little change of depth along the channel (Davies and Woodroffe, 2010). The convergence of those estuaries can be expressed in empirical relationships (Nienhuis et al., 2018), similar to those of rivers (Wilkerson and Parker, 2010). However, for the hydraulic geometry of the fluvial-tidal transition, which connects the river to the estuary, no empirical relationships have been formulated yet. A survey of the Mahakam showed, that the trends of downstream hydraulic geometry are not necessarily continuous along the fluvial-tidal transition (Sassi et al., 2012).

The geometry of rivers and estuaries can also be predicted with mathematical models. The challenge of morphodynamic modelling is to formulate a closed set of equations that determines all unknowns. Together with the conti- 
nuity of water and sediment transport, at least one more equation is required to determine width, depth and slope. Even more relations are required to determine roughness and grain size (Wright and Parker, 2005). In some models, one of the geometric properties, such as width or depth, is conveniently fixed, to circumvent the closure altogether (Bolla Pittaluga et al., 2015b; Wang and Ding, 2012). This is motivated by the funnel-shape of tidally dominated deltas (Wang and Ding, 2012; Savenije, 2012a). These models either enforce or predict a continuous downstream widening along the fluvial-tidal transition. However, observations in the Mahakam Delta also indicate, that tidal funnels with strong river inflow tend to deepen towards their upstream end (Sassi et al., 2012). A proper closure is thus required to predict the hydraulic geometry of the fluvial-tidal transition. For fluvial reaches, semi-empirical closures are applied that are based on extremal hypotheses, which assume minimum stream power, maximum energy dissipation or roughness (Phillips, 1991). However, it is unclear, if these apply along the fluvial-tidal transition.

So far there exists no unifying theory nor extensive empirical data for the trend of the hydraulic geometry along the fluvial-tidal transition, so that further evidence has to be collected in the field. Chapter 3 of this thesis presents the trends of hydraulic geometry and bed material along the fluvial-tidal transition of the Kapuas River.

\subsection{Acoustic surrogate measurement of sediment transport}

Channels are shaped by the deposition and the erosion of sediment. Differences thereof are linked to the net flux of sediment along the stream (Exner, 1925). Measurements of sediment transport therefore give insight into morphodynamics, and consequently into rates of delta progradation as well as the fate of delta channel branches. Sediment transport also gives direct insight into human-induced shifts in the sediment yield as well as its causes and consequences, such as elevated erosion rates due to deforestation (Buschman et al., 2012; Restrepo et al., 2015), coastal erosion due to mining of sand and trapping of sediment in reservoirs (Sreebha and Padmalal, 2011; Saviour, 2012; Kondolf et al., 2014), or damage to coral reefs by enlarged river plumes (Tarya et al., 2018).

Sediment transport strongly varies in space and time, due to its strong nonlinear dependence on the flow velocity. Although transport can be related to stage (Asselman, 2000), such a relation is much less reliable than stagedischarge rating. A direct measurement is therefore preferred. The sediment concentration is conventionally determined from water samples. How- 
ever, this method comes with large costs and effort and is unsuitable to resolve the variation in tidally influenced channels with complex geometries. The acoustic sensing of suspended sediment makes it possible to capture the spatio-temporal variation of the sediment concentration. So far, dedicated instruments for the acoustic monitoring of suspended sediment in rivers are not available, and the state-of-the-art is to opportunistically rely on ADCP backscatter (Guerrero et al., 2011). This has several drawbacks. Acoustic backscatter is sensitive both to the particle size as well as to the concentration of the suspended sediment (Guerrero et al., 2016). The inferred sediment concentration is therefore not only ambiguous, but also requires a specific calibration for each field site and flood stage (Sassi et al., 2012). Sound attenuation can further complicate the relation between backscatter and suspended sediment (Sassi et al., 2012). Chapter 5 explores the feasibility of acoustic measurement of the suspended sediment in the Kapuas River.

\subsection{Delta channel networks and their bifurcations}

Many deltas have a distributary channel network, where the flow takes more than one path towards the sea. These networks can be very dynamic, as individual channels can grow and dwindle, as long as their joint capacity remains the same. Even entirely new channels can form. During extreme flood events, the river can avulse anywhere along its backwater-influenced reach and then carve in a channel that meets the sea far from the existing outlets (Slingerland and Smith, 2004; Chatanantavet et al., 2012). New channels also gradually form by bifurcation of the flow around mouth bars during progradation of the delta (Edmonds and Slingerland, 2007; Canestrelli et al., 2014). At bifurcations, the along channel trend of the hydraulic geometry breaks. This is because the downstream branches have to be individually narrower and shallower, but together wider than the approaching channel, to ensure the continuous transport of both water and sediment. The bed level thus steps up towards both branches (Kerssens and Van Urk, 1986). Together, both branches are wider than the approaching channel, to ensure the continuous transport of sediment.

Changes of the discharge and sediment division at the delta bifurcations precede those of the channel morphology. In populated deltas, the discharge and sediment division ratio is therefore maintained for flood protection (Schielen et al., 2008), fresh water supply (Nguyen et al., 2008), shipping (Cooper, 2012), as well as to ensure the supply of sediment to particular coastal areas to mitigate coastal erosion (Allison et al., 2014). For this, it is advantageous that 
a bifurcation is intrinsically stable. A bifurcation is stable when the discharge and sediment supply to the branches equals the corresponding outflows, and the discharge and sediment division returns to this balanced state when the channel geometry is perturbed. This requires a branch that has been partially constricted to scour and a branch that has been widened to aggradate in response.

The division of discharge at bifurcations is determined by the relative flow capacity of the downstream branches. The flow capacity increases with the width and depth, but decreases with the length and roughness (Wang et al., 1995). The division of sediment at bifurcations is intrinsically more complicated as the division of water, as it is influenced by local factors such as the transversal variation of bed material grain size and secondary currents upstream of the bifurcation (Sassi et al., 2013; Frings and Kleinhans, 2008a). When the division is strongly simplified, then the evolution of the geometry of the branches over time can be modelled by a set of ordinary differential equations.

Wang et al. (1995) formulated a model with fixed channel widths, where both the sediment transport and division are expressed as power laws of the discharges of the branches. With this model, the stability of a bifurcation depending on the powers of the discharge division and sediment transport can be determined. However, the branches of stable bifurcations predicted by this model are of equal depth, even when the width of the branches differs. This contradicts the empirical hydraulic geometry, which predicts that narrower channels are also shallower. In combination with the relations of empirical hydraulic geometry, the model can be reformulated to allow also for the change of width (Mosselman, 2017). However, in this case, all stable bifurcations are of equal width and depth, which contrasts the observation that many natural bifurcations are asymmetric, i.e. have branches of different dimensions (Edmonds and Slingerland, 2008). Bolla Pittaluga et al. (2003) formulated a model that accounts for the effect of the transverse slope across the approaching channel for the case that the downstream branches are of different depth. As sediment rolls down the transverse slope, more sediment is diverted to the deeper channel. This model is able to predict stable asymmetric bifurcations for cases where the width-to-depth ratio of the approaching channel exceeds a threshold which is typical for gravel-bed rivers (Bertoldi and Tubino, 2007). An extension of the model to sand bed rivers (Bolla Pittaluga et al., 2015a) and numerical simulations (Edmonds and Slingerland, 2008) showed that the stability depends on the critical shear stress, and that stable asymmetric bifurcations can exist when the critical shear stress is either low, as is the case 
in gravel-bed rivers, or very high, as in large sand-bed rivers. The role of the aspect ratio is also confirmed by analytical models (Redolfi et al., 2016).

The stability of bifurcations can also be strongly influenced by meandering of the river, as the branch that is located in the outer bend receives less sediment than that located in the inner bend (Kleinhans et al., 2008). Further factors, like steps in bed level to smaller branches (Slingerland and Smith, 1998), bed material sorting (Sloff and Mosselman, 2012) and variation of the river stage (Edmonds et al., 2010) all influence the division of sediment.

Tides certainly affect the morphology of bifurcations, but little is known except for more general observations like that that tides deepen channels (Shaw and Mohrig, 2014) and promote scour (Ferrarin et al., 2018). It has been suggested, that tides stabilise delta bifurcations (Hoitink et al., 2017). The hydrodynamics of channel networks is better understood. Tidal waves are partially reflected and transmitted at bifurcations. Models for tidal propagation in delta channel networks have been developed by Hill and Souza (2006) and Alebregtse and de Swart (2016). Tides can also influence the tidally averaged discharge division, by raising the tidally averaged water elevation and by residual Stokes transport. Depending on local conditions, tides can either favour the division of water to the larger (Buschman et al., 2010) or to the smaller (Sassi et al., 2011a) branch.

\subsubsection{Strongly asymmetric bifurcations}

Another important factor that influences the division of sediment is the planform of a bifurcation. Many river bifurcations have downstream branches that different in size so that the discharge division is not symmetrical. At such a bifurcation the larger branch typically continuous straight in the direction of the approaching channel, while the smaller branch splits off to the side. This is similar to engineered lateral diversions which have been extensively studied (Raudkivi, 1993). An early experimental result was that a relatively large fraction of sediment is diverted into the side branch. This is referred to as the Bulle effect, named after one of the early experimentators who observed this in flume experiments (Bulle, 1926). The effect has been reproduced in numerous physical and numerical experiments (Kawai et al., 1993; Riad, 1961; Dutta et al., 2017; Ksiazek and Meijer, 2011). Sediment at such diversions is divided differently than predicted by simple models, like that of Wang et al. (1995).

The excess of the diverted sediment is caused by the secondary flow. As the flow is curving into the side branch, a flow cell forms which directs a larger 
fraction of water from the lower part of the water column than from the higher part of the water column into the side branch. This does not affect the total amount of diverted water, but increases the amount of the diverted sediment, as the sediment concentration is higher near the bed than near the surface (Raudkivi, 1993). The strength of the effect is lower when the sediment is transported in suspension than when it is transported as bed load, but it is still considerable, as the concentration of suspended load increases exponentially towards the bed (van Rijn, 1984b). A small side branch that receives a large fraction of the sediment will thus rapidly fill up. However, this contrasts the observation that many asymmetric bifurcations are stable (Axelsson, 1967). Thus, other factors seem to compensate Bulle's effect in particular situations. The sediment to discharge division ratio becomes small when only a small fraction of the discharge is diverted to the side branch (Dutta et al., 2017; Gaweesh and Meselhe, 2016). The strength of the Bulle effect therefore decreases over time as the side branch aggradates (Matthes, 1933). This also agrees with the observation of asymmetric bifurcations. The morphodynamics of a bifurcation is not necessarily well described by the branches as a whole, as local sedimentation can plug a branch at the upstream end even when the overall geometry of that branch changes little (Miori et al., 2006; Dargahi, 2004).

The secondary flow also strongly affects the bed geometry. The flow curves twice. At the upstream part of the bifurcation, it curves towards the side branch and at the downstream end of the bifurcation, the remaining flow curves back into the direction of the main channel. There are thus two counterrotating secondary flow cells. These scour the bed at the splitting-point at the downstream end of the bifurcation and cause the formation of two bars, one at the upstream bank of the side branch and one in the main channel at the bank opposite to the side channel (Neary et al., 1999). In extreme cases, the flow in the main channel can reverse at the downstream end of the inlet to the side channel, and the flow can separate over the bars. The recirculation bubble caused by flow separation in the side branch can reduce the effective inlet width and the amount of the diverted water and sediment (Constantine et al., 2010). The secondary flow leading into the side branch leads to erosion of the downstream bank of the inlet to the side channel, and aggradation on the opposite bank, i.e. the upstream bank of the inlet to the side channel. If the aggradation is more rapid than the erosion of the outer bank, then the inlet of the side branch is gradually closed off. This is how oxbow lakes form after a meander neck cut-off. Vegetation growth on the bar can accelerate this process, especially when the water level varies seasonally.

The division of sediment at natural bifurcations is sensitive to many fac- 
tors, and can only be well understood with measurements. Neither simple semi-empirical relations, nor physical models are necessarily representative for bifurcations found in nature, as they lack important details such as complex channel geometries, suspended load transport, bed material sorting and tides. However, studies of bifurcations in large sand bed rivers are rare (Edmonds and Slingerland, 2008; Kleinhans et al., 2013). Syvitski et al. (2005b) pointed out that although distributary channels determine the morphology of deltas, there are only few studies on them, due to the high cost and complexity. Chapter 6 presents measurements of the discharge and sediment division of two strongly asymmetric bifurcations in the Kapuas River and chapter 7 shows the consequences for theoretical modelling of river bifurcations.

\subsection{The Kapuas River research project}

Rivers in the humid tropics are those with the largest discharges and sediment loads of the world. Their evergreen and everwet catchments are hotspots of biodiversity and their fertile deltas are acres of plenty for the production of rice, palm oil and rubber. At present, tropical rivers, their catchments, and deltas face growing pressure from rapid economic development and climate change, which may permanently deteriorate their ecosystem services. Yet, despite their importance and advancing degradation, relatively little is known about their physiography. This thesis reduces this gap by contributing to our fundamental understanding of tropical rivers.

This thesis is part of the joint Indonesian-Dutch project Hydrology-Geomorphology Links in the Kapuas River System. It aims to improve the understanding of hydrology and morphology of tropical rivers and their wetlands. The project consists of four parts. These are:

1 Multi-scale Modelling of the Kapuas Land-Sea Continuum (this thesis) has the goal to improve the understanding of the hydrodynamics and morphology of tidal rivers. This project follows up on: Discharge Regimes, Tides and Morphometry in the Mahakam Delta Channel Network (Sassi, 2013).

2 River Morphology in Peat Environments has the goal improve the general understanding of the morphology, flow and turbulence in sharp river bends. Projects 1 and 2 conducted their field survey jointly. Project 2 follows up on: Rivers Running Deep: Complex Flow and Morphology in the Mahakam River (Vermeulen, 2014). 
3 Hydrology of the Kapuas catchment has the goal to improve the understanding of the hydrological cycle in large tropical catchments, its resilience to land use, as well as to climate change. Particular attention is paid to the role of the Danau Sentarum lake area on the hydrological regime as well as its vulnerability to human intervention (Hidayat et al., 2017). The study area of project 3 is the non-tidal river upstream of Sanggau, while the study area of this thesis is the tidal river further downstream. The discharge gauged at Sanggau by project 1 is used by project 3 to calibrate a rainfall-runoff model. Project 3 follows up on Runoff, discharge and flood occurrence in a poorly gauged tropical basin: The Mahakam River, Kalimantan, (Hidayat, 2013).

4 Hydrological Drought and Wildfire in the Humid Tropics (Taufik, 2017) focusses on the propagation of drought from rainfall to streamflow (Taufik et al., 2015), drought as a precondition of wildfires (Taufik et al., 2017, 2018b), as well as their aggravation by human interference (Taufik et al., 2018a). Projects 3 and 4 conducted their field surveys of the headwaters jointly.

\subsection{Characteristics of the Kapuas River and its catchment}

The basin of the Kapuas River covers the western part of the island of Borneo (Kalimantan) and is the largest river in the Indonesian archipelago. Local communities depend on the river for transportation, fishing and as a freshwater source. The Kapuas is ideal to study the hydrodynamics and morphodynamics of a natural river delta: West Kalimantan is only sparsely populated, the main delta lobe is not dredged, sand mining is limited, and the river has not yet been constrained by dams, dykes or groins, as has historically been the fate of European rivers (Tockner and Stanford, 2002) and more recently of those on the Asian mainland (Brunier et al., 2014). Yet the Kapuas cannot be considered entirely pristine: most of the lowland forest has been cleared from Borneo during the last decades (Gaveau et al., 2014), and vast areas of peatland have been drained to make space for oil-palm cultivations (Anshari et al., 2010), which has disturbed the hydrological regime (Kumagai et al., 2013b) and likely the sediment input. A survey of the Kapuas can also aid future river management, as the discharge and water levels of the river are not systematically monitored. An extensive treatment of the Kapuas can be found in Enthoven (1903). The following sections only give a short overview and references to recent contributions to the literature. 


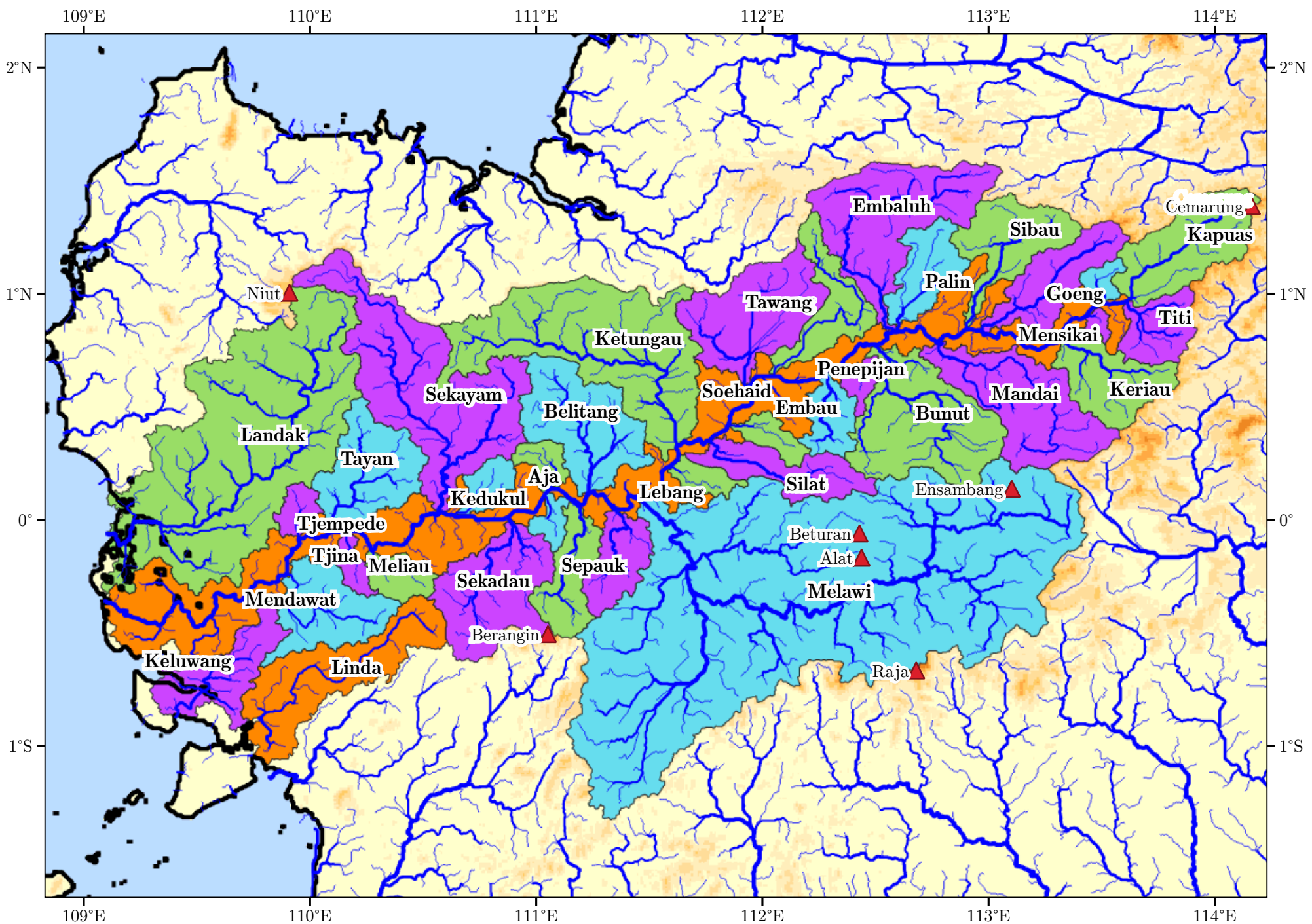

Figure 1.5: Tributary catchments of the Kapuas River 


\subsubsection{Topography and geology}

The basin of the Kapuas River spans from East to West over a distance of $600 \mathrm{~km}$ and extends over a total catchment area of $99000 \mathrm{~km}^{2}$ (Hidayat et al., 2017). Its main source is near Mount Cemaru, a mountain in the centre of the island, with a summit of $1684 \mathrm{~m}$ above sea level (figure on front cover). About $1100 \mathrm{~km}$ from the source, the Kapuas discharges into the Karimata Strait. The Karimata strait is shallow and located on the continental margin. It separates Borneo from the island of Sumatra in the East and connects the South China Sea to the North, and the Java Sea to the South.

Borneo's river network took its present form 4 million years ago, following the uplift of the central mountain range and the separation of the Melawi and Kutai (Mahakam) basins during the late Oligocene and early Miocene (van Bemmelen, 1949; Moss and Wilson, 1998; Wilson and Moss, 1999; Hall and Nichols, 2002). The central mountain range of Borneo, where the Kapuas has its source, consists mostly of Cretaceous marine sediments. The Kapuas leaves the mountains near Lunsa, $850 \mathrm{~km}$ from the sea, and enters the Ketungau basin, which is filled with Neogene fluvial sediments. The gravel-sand transition occurs most likely here, at the foot of the mountains (Fehn, 1933). In the Ketungau Basin, the valley widens up to form a large floodplain, in which Danau Sentarum lake area is located. Downstream of Semitau, $610 \mathrm{~km}$ from the sea, the river leaves the floodplain and breaks through the Semitau mountain range, which consists of Oligocene volcanic rocks. Beyond the mountain range, the Kapuas enters the Melawi basin, which is likewise filled with Neogene fluvial sediments (Sutjipto, 1991). At Sintang, $460 \mathrm{~km}$ upstream from the sea, the Kapuas meets the Melawi river, its largest tributary. Between Meliau and Tajan, the Kapuas breaks through the Schwaner mountain range, which consists of Cretaceous plutonic rocks. Along the last $200 \mathrm{~km}$ downstream of Tajan the Kapuas flows through the delta plain, which consists mostly of Neogene fluvial sediments and quaternary sediments recently deposited by the Kapuas.

The elevation profile of the Kapuas is unusual, as its bed level does not gradually rise (figure 1.6). The bed level rises steadily until Sintang, $420 \mathrm{~km}$ from the sea, but thereafter hardly rises until Bunot, $850 \mathrm{~km}$ from the sea. This is because the Kapuas river has changed its course geologically recently (Sibinga, 1953) so that there has not yet been sufficient time for the Kapuas to carve a graded profile. Initially, the Kapuas drained what is today the Semitau lake area to the North-West, and flowed along a shorter route along what is today the Lupar river towards the South-China sea. Since then the Kapuas has 
broken through the Semitau range and made the lower reach of the Melawi river its own. The elevation profile downstream of Sintang indeed resembles the continuation of the Melawi profile (figure 1.6). The change of course is also evident by the path of the Lupar river, which takes a right turn at its former meeting point with the proto-Kapuas (figure on inside back cover). This former junction is covered by the Batang Ai reservoir since 1985 and is even apparent in the reservoir's shoreline.

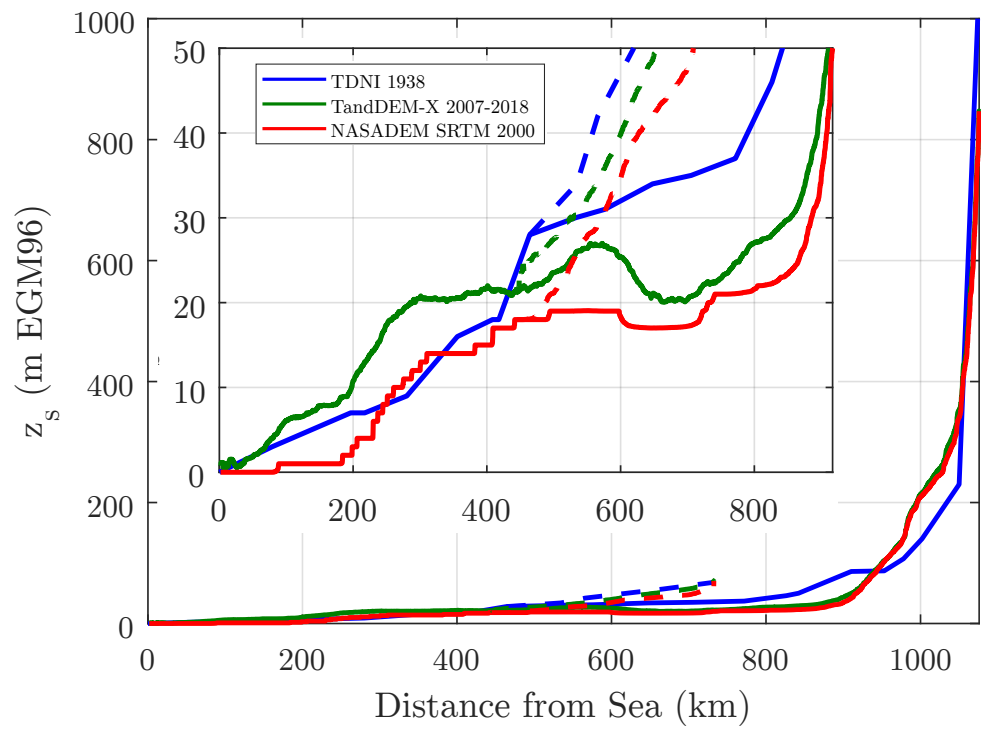

Figure 1.6: Elevation profile of the Kapuas River (solid) and the Melawi tributary (dashed), from a historic map and satellite measurements; inset zooms into the lowland reaches

Within the Kapuas Hulu lake area, the Kapuas actively meanders, so that numerous oxbow lakes are found. This is in stark contrast to the coastal plain, where the banks migrate slowly and no oxbow lakes are found (Vermeulen et al., 2016). The only evidence of recent activity in the coastal plain are two avulsion loops and nearby scroll bars at the upstream end of the coastal plain. The minor branches of the loops have considerably filled over the last century. The Kapuas Besar thus forms a single meandering channel, besides three inferior distributaries which branch off along the alluvial plain. The main stem of the river is called the Kapuas Besar, while the distributaries are called the Mendawat, the Kapuas Kecil, and Kubu. The Kapuas Besar forms a small radial delta with five outlets and a radius of $15 \mathrm{~km}$. These outlets have the shape of small tidal funnels. The radial delta has considerably prograded into the sea over the last century (figure 1.7), throughout which cyclically mouth bars emerged and merged with older delta lobes. The Kapuas Kecil and Kubu 
also terminate in small tidal funnels. The funnels of all distributaries are separated from the sea by shallow bars. The Kapuas Kecil is the largest distributary and the only distributary that branches off to the North. It is met by the Landak river $20 \mathrm{~km}$ upstream from the sea, where the regional capital Pontianak is situated (570000 inhabitants). Downstream of Tajan, there are only a few small settlements at the Banks of the Kapuas Besar. Both the Mendawat and Kubu connect to an extensive tidal creek network to the South of the Kapuas. Including the tidal creek network, the Kapuas delta extends from north to south over a distance of more than $140 \mathrm{~km}$ as the crow flies. The Kapuas delta has formed geologically recently since the end of the last glaciation 15 thousand years ago. As the Karimata street is shallow, the coastline retreats during glacial periods by more than $880 \mathrm{~km}$, so that Borneo becomes part of mainland Asia to join the delta plain of the Mekong river (Voris, 2000). This is different from the Mahakam Delta in East Borneo, where the coast retreats little during glaciation, as the shelf edge is only $30 \mathrm{~km}$ offshore.

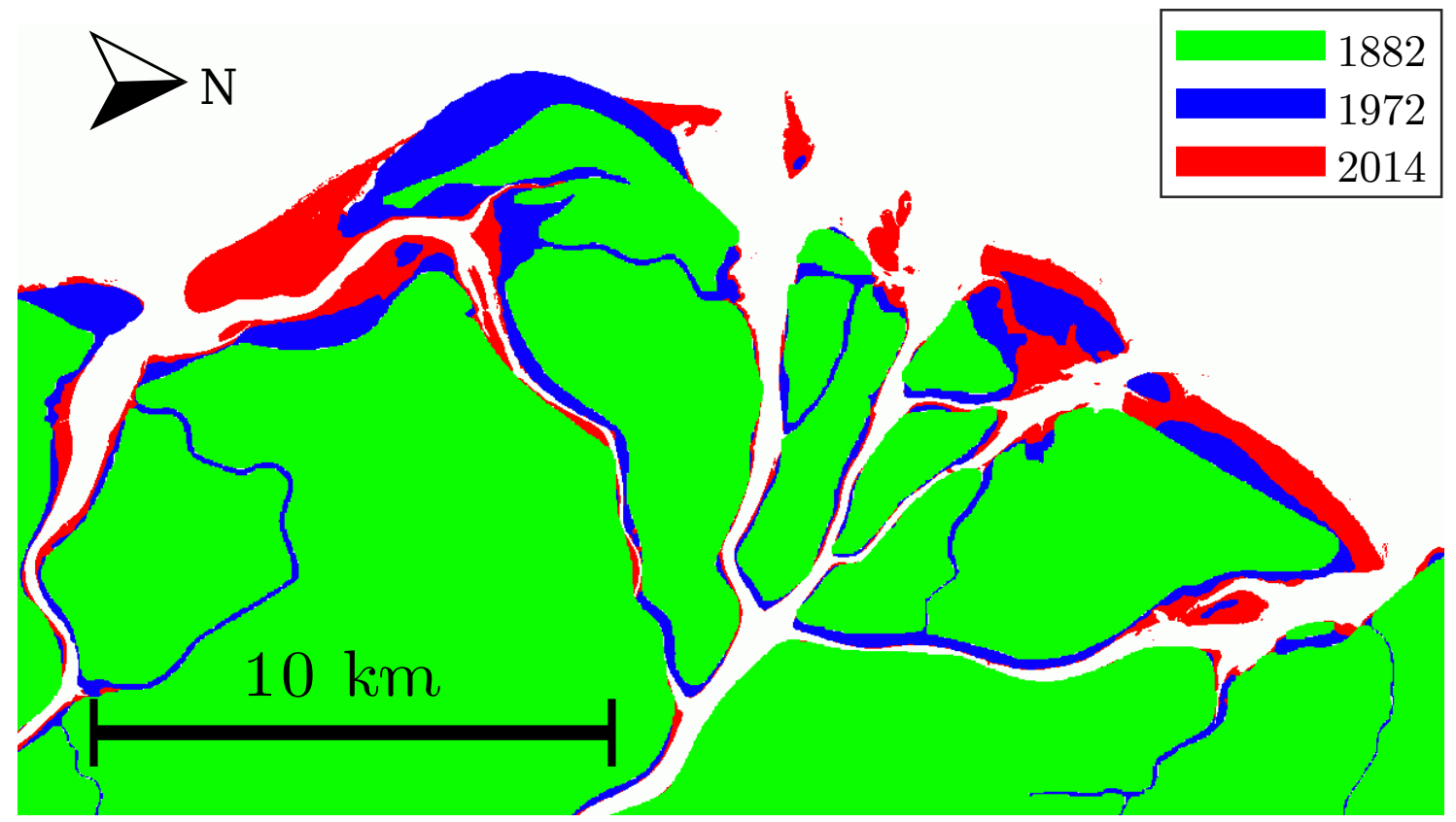

Figure 1.7: Kapuas Besar delta lobe 1882, 1972 and 2014, (Hydrograaf, 1907) and Landsat (courtesy of the USGS)

\subsubsection{Hydrology}

As the basin of the Kapuas River is situated in the humid-tropics and crossed by the equator. The weather is seasonally varied by the monsoon. The wettest month is December with $350 \mathrm{~mm}$, the driest months are July and August with 
$220 \mathrm{~mm}$ (figure 8.1) (Hidayat et al., 2017). During the wet season, the Kapuas carries large amounts of driftwood, that amasses to a continuous band of logs along streamline of highest velocity.

A rapid response and heavy orographic rains cause frequent floods(banjirs) and high erosion in the headwater tributaries (Fehn, 1933). These floods typically last less than a day and most tributaries are only turbid during these floods, while the main river is year-round turbid. The downstream part of tributary valleys is flooded by backwater from the main river during the wet season. These trap sediment and form blackwater habitats. Extensive logging and mining has turned many of the tributaries year-round turbid, as elsewhere on Borneo (Douglas, 1996; Yule et al., 2010; Luke et al., 2017).

During the wet season, the land surrounding the Danau Sentarum lakes floods. The lakes slightly reduce discharge peaks and buffer during the falling limb (Bol, 2015). Although it is in unclear to which degree the flood waves and sediment bypass the lake area in the main channel and at which stage peaks are buffered and respectively accommodated in the lake area due to overbank flow. The lakes are connected to the Kapuas by a tie channel, and fall completely dry in some years. About half of the discharge joins the Kapuas downstream of the Danau Sentarum lake area, including the discharge of its largest tributary, the Melawi (Bol, 2015). The lakes therefore only partially influence the discharge reaching the delta.

van Bemmelen (1949) states that the annual discharge of the Kapuas as $6.510^{3} \mathrm{~m}^{3} / \mathrm{s}$. The WBMsed model (Cohen et al., 2013) gives an average discharge of $5.510^{3} \mathrm{~m}^{3} / \mathrm{s}$ with average seasonal extrema of $8.910^{3} \mathrm{~m}^{3} / \mathrm{s}$ and 2.0 $10^{3} \mathrm{~m}^{3} / \mathrm{s}$. This is in contrast to Milliman and Farnsworth (2013) based on Meybeck and Ragu (1995), who state an annual discharge of the Kapuas of only 3.2 $10^{3} \mathrm{~m}^{3} / \mathrm{s}$. Differences may partly stem from different delineations of the catchment within the delta. Only smaller tributaries join the Kapuas downstream of Sanggau. Change of climate and land use can change precipitation and discharge regime. Kumagai et al. (2013a) found that discharge over Borneo decreased from $2600 \mathrm{~mm} / \mathrm{a}$ to merely $1900 \mathrm{~mm} / \mathrm{a}$ due to land use change. However, Takahashi et al. (2017) does not support this finding and states that the influence of deforestation is uncertain. Even if correct in general, the findings of Kumagai et. al do not need to apply to the Kapuas catchment in particular, as it encompasses of $1 / 5^{\text {th }}$ of Borneo's area and the rainforest in its mountainous headwaters remains relatively intact compared to that of Sarawak, the Malaysian part of Borneo. Herawati et al. (2017) found that precipitation in the Kapuas catchment has increased by $4.3 \%$ between 2002 and 2012, and that discharge is not corresponding to rainfall very well, which they attribute 
to changes in the land use. However, their analysis is based on a simple correlation of rainfall measured at the coastal station Supadio near Pontianak and only 10 years of water levels gauged in Sanggau.

Direct measurements of discharge (chapter 2) and consecutive rainfall-runoffmodelling within the framework of project 3 (Hidayat et al., 2017) shed more light onto the discharge regime and climatological variability of the Kapuas River.
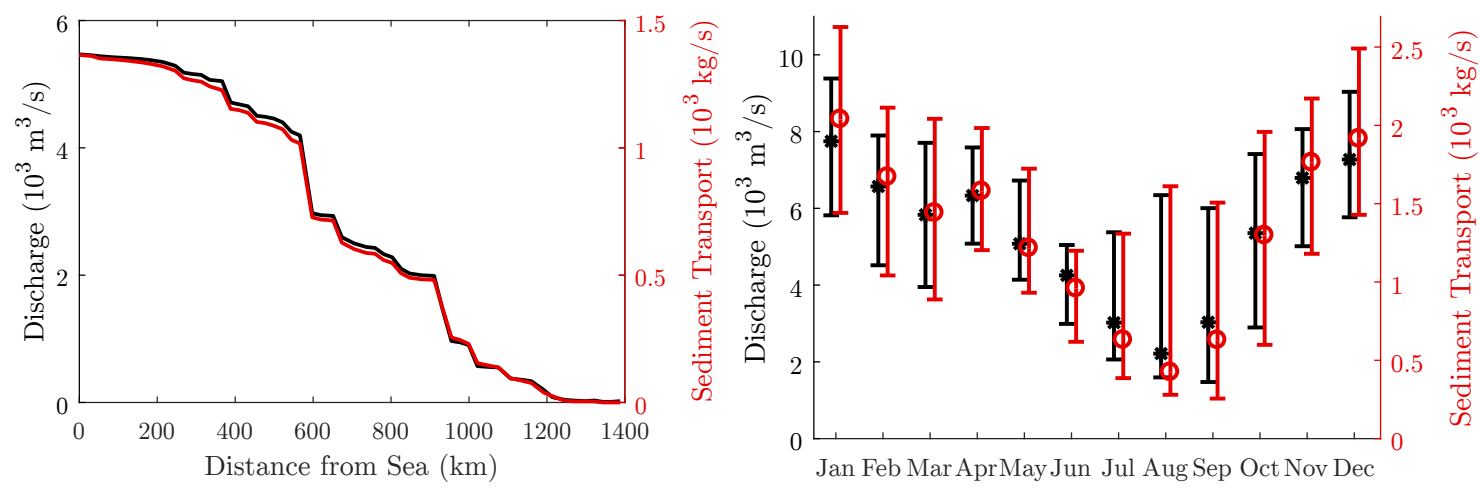

Figure 1.8: Discharge and sediment transport of the Kapuas River between 1980-2010 from the WBMsed database at the river mouth (WBMsed does not include delta bifurcations), a) along the river b) median for each month over the year, bars indicate the $16^{\text {th }}$ and $84^{\text {th }}$ percentiles

\subsubsection{Tides}

The Kapuas is set in a microtidal regime, with a mean spring range of $1.45 \mathrm{~m}$ at its river mouth. The tidal regime is mixed, mainly diurnal (figure 1.9). The small size of the tidal funnels indicates that the tides only dominate the flow for a short distance of approximately $15 \mathrm{~km}$ from the sea so that the bifurcations of the Kapuas are tidally influenced, but river-dominated. The morphology undergoes a remarkable change along the coastline. In the North, there are no larger tidal creeks, while a vast network of tidal creeks extends to the South. This might be related to shifts in the tidal regime that becomes more diurnal towards the South (Wei et al., 2016).

\subsubsection{Sediment yield}

Rivers in insular South-East Asia deliver more sediment to the ocean than those in any other part of the world (Milliman et al., 1999; Hall and Nichols, 2002). Yet, sediment transport has hardly been measured, let alone be systematically monitored. Milliman et al. (1999) estimated the sediment yield 

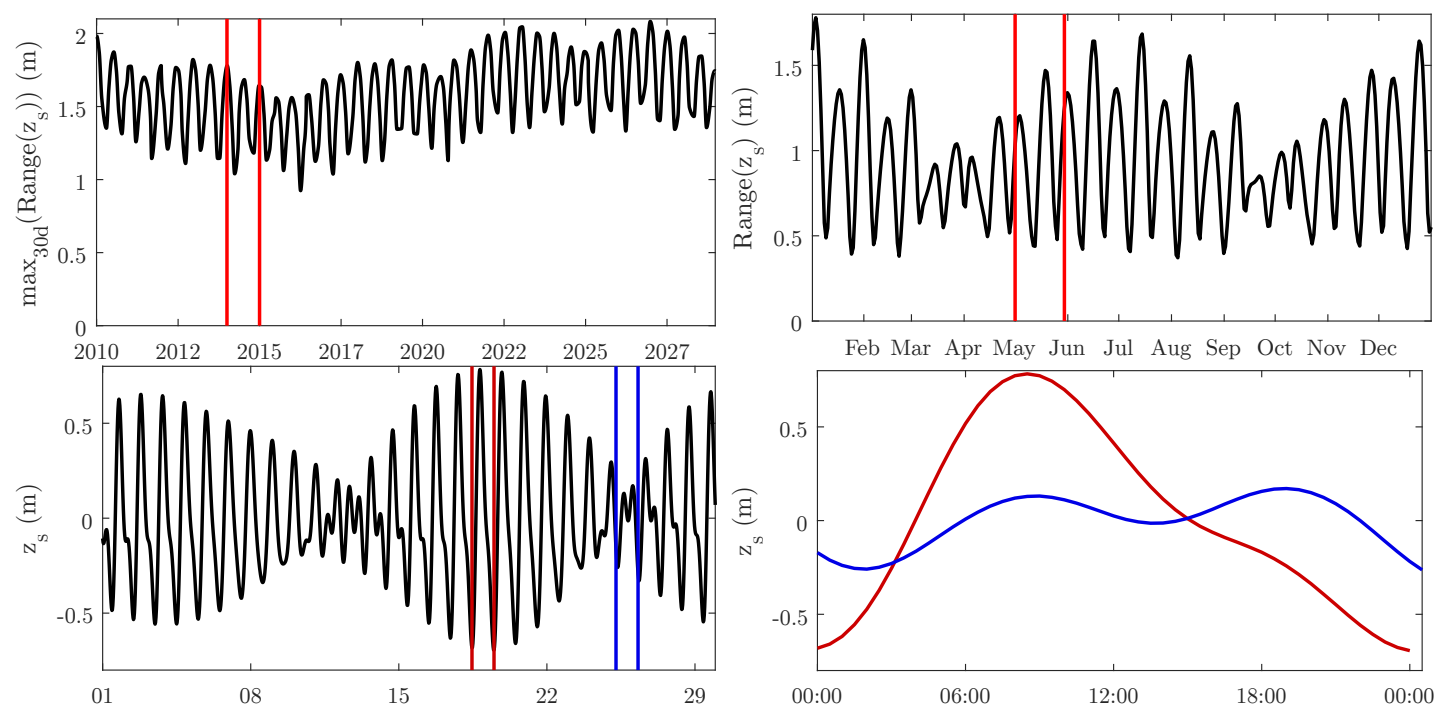

Figure 1.9: Tidal regime at the Kapuas river mouth: range over the Metonic and annual cycle and water levels over the monthly, as well as daily cycles during spring and neap tide (TPXO prediction (Egbert and Erofeeva, 2002))

for Borneo with reference data from Java, although both islands have an entirely different geology. Java is of volcanic origin and has mostly short rivers with steep slopes, whereas neither is the case for Borneo. Even on Borneo itself, sediment transport strongly varies between different parts of the island. Erosion and sediment delivery are high in the north-west of Borneo, like the Rajang catchment (Douglas et al., 1999; Staub and Gastaldo, 2000), where deforestation has already progressed to higher altitudes. In contrast, sediment loads are moderate in the eastern parts like the Berau (Buschman et al., 2012) and Mahakam basins (Sassi et al., 2013; Van et al., 2016), that still have intact forest covers in their head-waters. Sediment delivery predicted by the WBMsed model are on average $1.4,10^{3} \mathrm{~kg} / \mathrm{s}$ with seasonal extrema of $0.410^{3} \mathrm{~kg} / \mathrm{s}$ and $2.410^{3} \mathrm{~kg} / \mathrm{s}$. This indicates high concentrations $\left(0.25 \mathrm{~kg} / \mathrm{m}^{3}\right)$ and a low degree of non-linearity of the sediment transport, which is typical for wash-load dominated transport.

Rapid development alters the sediment transport regimes in Southeast-Asia (Syvitski et al., 2005). On one hand, mining and deforestation lead to high erosion and land degradation. This increases the turbidity of the river as well as the coastal sea and thereby negatively affects ecosystems from streams to coral-reefs (Yule et al., 2010; Tarya et al., 2018). On the other hand, damming and sand mining deprive deltas of sediment and lead to excessive coastal erosion. How far the sediment transport regime of the Kapuas river is already affected is not clear. 
Chapters 5 and 6 give a first insight into the sediment transport regime in the coastal reaches of the Kapuas river, along with a calibration for the acoustic measurement of suspended sediment.

\subsection{Research questions and thesis outline}

The subsequent chapters in this thesis address the following research questions:

\section{Chapter 2: Discharge monitoring in tidal rivers}

- Under which conditions are HADCPs suitable for discharge monitoring?

- What are the sources of uncertainty and how can they be by quantified?

\section{Chapter 3: Morphology of the fluvial-tidal transition}

- What are the trends of hydraulic geometry and bed material along the fluvial tidal transition?

- How comparable is the hydraulic geometry and bed material between the distributaries of one delta?

\section{Chapter 4: Propagation of tides up-river}

- How does the backwater dynamics due to the sloping bed and varying discharge influence the propagation of the tide?

- Where is the fluvial-tidal transition and how far beyond does the tide propagate up-river?

\section{Chapter 5: Acoustic surrogate measurements of suspended sediment}

- What are the advantages and limitations of acoustic measurements of suspended sediment in large sand-bed rivers?

- What is the best practice to infer sediment concentrations from monofrequency ADCP backscatter?

\section{Chapters 6 and 7: Discharge and sediment division at delta bifurca- tions}

These two chapters address questions regarding tidally influenced delta bifurcations in large sand bedded rivers: 
- What is the bed morphology?

- How are discharge and sediment divided among the branches?

- Which processes govern the division? 


\section{Prerequisites for accurate monitoring of river discharge based on fixed location velocity measurements}

$\mathrm{R}^{\text {iver discharge has to be monitored reliably for effective water manage- }}$ $\boldsymbol{R}$ ment. As river discharge cannot be measured directly, it is usually inferred from the water level. This practice is unreliable at places where the relation between water level and flow velocity is ambiguous. In such a case, the continuous measurement of the flow velocity can improve the discharge prediction. The emergence of horizontal acoustic Doppler current profilers (HADCPs) has made it possible to continuously measure the flow velocity. However, the profiling range of HADCPs is limited, so that a single instrument can only partially cover a wide cross section. The total discharge still has to be determined with a model. While the limitations of rating curves are well understood, there is not yet a comprehensive theory to assess the accuracy of discharge predicted from velocity measurements. Such a theory is necessary to discriminate which factors influence the measurements, and to improve instrument deployment as well as discharge prediction. This paper presents a generic method to assess the uncertainty of discharge predicted from rangelimited velocity profiles. The theory shows that a major source of error is the variation of the ratio between the local and cross section averaged velocity. This variation is large near the banks, where HADCPs are usually deployed and can limit the advantage gained from the velocity measurement. We apply our theory at two gauging stations situated in the Kapuas River, Indonesia. We find that at one of the two stations the index velocity does not outperform a simple rating curve.

This chapter is based on: Kästner, K., A. J. F. Hoitink, P. J. J. F. Torfs, B. Vermeulen, N. S. Ningsih, and M. Pramulya, Prerequisites for accurate monitoring of river discharge based on fixed-location velocity measurements, Water Resources Research, 54(2), 1058-1076, 2018 


\subsection{Introduction}

In theory, river discharge can be monitored more accurately when the flow velocity of the water is directly measured, instead of being inferred from the water level. As the cost and effort required to deploy a velocity meter greatly exceed those of a simple water level gauge, velocity meters are usually deployed only at locations where there is no simple relation between water level and flow velocity. These locations include water bodies influenced by tides (Bradley, 1999; Hoitink et al., 2009; Sassi et al., 2011b) and backwater (Hidayat et al., 2011; Jackson et al., 2012). It is important to assess if a deployment achieves the quality required by the water management (Muste and Hoitink, 2017).

For continuous monitoring of river flow, horizontal acoustic Doppler current profilers (HADCPs) are increasingly being deployed. ADCPs are acoustic instruments that determine the flow velocity from the reflection of sound by suspended particles moving with the flow (Gordon, 1989). With increasing distance from the instrument, the signal is attenuated and reflections from the bottom and water surface interfere with the signal. Both effects limit the range over which an HADCP can measure, so that wide channels cannot be covered by a single instrument. In addition, HADCPs measure only at a single depth.

If the velocity is only measured across part of the channel, then the cross section averaged velocity has to be inferred with an appropriate method. The relation between local and cross section-averaged velocity is determined by the spatial velocity distribution within the cross section. The velocity distribution depends on channel geometry, curvature, and water level. It is highly sensitive to perturbations near the river bed and the embankment. The spatiotemporal variation of the velocity distribution limits the accuracy of the discharge prediction, as it is not entirely predictable. The accuracy that can be achieved depends on the location, orientation, and profiling range of the instrument. Flow velocity meters also require a rigid deployment, a free line of sight, and an external power supply, which makes them prone to malfunction. The deployments of flow meters therefore require careful site specific planning and calibration. This process could benefit from a comprehensive theory that allows to evaluate the performance of HADCPs for discharge monitoring. However, such a theory is not yet available.

This paper develops a statistical method to determine the accuracy of discharge monitored with HADCPs depending on the deployment specific conditions (section 2.2). In particular, it elaborates on the sensitivity of the pre- 
dicted discharge with respect to variation of the vertical and the transverse profile of the streamwise velocity. We apply this method in section 2.5 at two field sites located along the Kapuas River, Indonesia. Complex variation of the velocity profile and relatively short profiling ranges limit the accuracy of the discharge monitored at both stations. We find that the HADCP measurements are essential to monitor the discharge at the downstream gauging station, which is affected by tides and backwater from the sea. However, at the upstream station, where the discharge is well predicted by a rating curve, the HADCP measurements have little added value. We discuss physical processes that affect the velocity distribution at both field sites, and give recommendations for future HADCP deployments and discharge prediction.

\subsection{Theory of discharge predicted from range limited velocity profiles}

Section 2.2.1 explains the principle of determining discharge from a velocity measurement. Based on this principle, sections 2.2.2 and 2.2.3 develop error estimates for discharge determined from point and range-averaged velocity measurements. Section 2.4.3 describes the principle of determining discharge with rating curves.

\subsubsection{Determining discharge from velocity measurements}

Discharge $Q$ is the product of the cross section area $A$ and the average velocity component $U$ that is perpendicular to the cross section,

$$
Q=A U
$$

$U$ cannot be directly measured but can be estimated by measuring the local velocity $u$ at sufficiently many points in the cross section and averaging it. If the flow velocity is only measured in a small part of the cross section, then the spatial distribution of the velocity has to be known, to determine the cross sectionally averaged velocity $U$.

At any vertical across the channel, the depth-averaged velocity $\bar{u}$ is determined by the form function $f_{t}$ :

$$
\bar{u}=f_{t} U \text {. }
$$

Likewise, the velocity $u$ is determined by the form function $f_{v}$ for any point along a vertical:

$$
u=f_{v} \bar{u} .
$$


The shape of $f_{v}$ can vary over the cross section.

Thus, if the values of the normalized velocity profiles $f_{t}$ and $f_{v}$ at the point of measurement are known, then the discharge $Q$ can be determined as

$$
Q=A \frac{1}{f_{t}} \frac{1}{f_{v}} u
$$

for any point where the water is not stagnant.

\subsubsection{Mean squared error of the predicted discharge}

In general, the quantities entering equation (2.4) are not known exactly, and instead of determining the discharge $Q$ exactly, it is only possible to estimate it as $\hat{Q}=\hat{A} \frac{1}{\hat{f}_{t}} \frac{1}{\hat{f}_{v}} \hat{u}$, where $\hat{u}$ is the measured velocity, $\hat{A}$ the predicted cross section area, and $\hat{f}_{t}$ and $\hat{f}_{v}$ are coefficients based on the modeled velocity profile. As for continuous measurements with a rigidly deployed instrument the monitoring locations are fixed, the values of $\hat{f}_{t}$ and $\hat{f}_{v}$ represent calibration coefficients. The value of these coefficients can vary depending on the flow situation. Any error in the measured and modeled quantities contributes to the error $\varepsilon_{\hat{Q}}$ of the discharge estimate, which is the difference between the predicted and measured discharge $\left(\varepsilon_{\hat{Q}}=\hat{Q}-Q\right)$. The prediction error can only be determined for moments in time when a reference measurement is available. However, the mean-squared-error can be estimated for any moment in time. To do this, we consider the function $\mathcal{Q}$ that predicts the discharge $\hat{Q}$. The sensitivity of this function with respect to the individual quantities is given by series expansion as:

$$
\hat{Q}=Q+\varepsilon_{t} \frac{\partial \mathcal{Q}}{\partial f_{t}}+\varepsilon_{v} \frac{\partial \mathcal{Q}}{\partial f_{v}}+\varepsilon_{A} \frac{\partial \mathcal{Q}}{\partial A}+\varepsilon_{u} \frac{\partial \mathcal{Q}}{\partial u}+O\left(\varepsilon_{t}^{2}, \varepsilon_{v}^{2}, \varepsilon_{u}^{2}, \varepsilon_{A}^{2}\right)
$$

where $\varepsilon_{t}=\hat{f}_{t}-f_{t}$ and $\varepsilon_{v}=\hat{f}_{v}-f_{v}$ are the errors of the modeled transverse and vertical profile coefficients. The quantities $\varepsilon_{A}=\hat{A}-A$ and $\varepsilon_{u}=\hat{u}-u$ are the errors of the predicted cross section area and of the measured flow velocity. The evaluation of the partial derivatives of $\mathcal{Q}$ yields

$$
\hat{Q}=Q\left(1-\frac{\varepsilon_{\hat{f}_{t}}}{f_{t}}-\frac{\varepsilon_{v}}{f_{v}}+\frac{\varepsilon_{\hat{A}}}{A}+\frac{\varepsilon_{\hat{u}}}{u}\right)+O\left(\varepsilon_{t}^{2}, \varepsilon_{v}^{2}, \varepsilon_{\hat{u}}^{2}, \varepsilon_{\hat{A}}^{2}\right) .
$$

Hereafter, the analysis only considers the relative error in the coefficients $\hat{f}_{v}$ and $\hat{f}_{t}$, as these are the most important sources of error in the discharge estimate. The error in the area is neglected, because it can be reliably predicted 
as long as the cross section is morphologically stable. The error in the measured flow velocity is neglected, as in a fixed deployment the noise can be reduced by averaging the measurement over time.

The error variance $\sigma_{\hat{Q}}^{2}$ of the predicted discharge can be estimated from the error variances of the profile coefficients, $\sigma_{t}^{2}$ and $\sigma_{v}^{2}$ :

$$
\sigma_{\hat{Q}}^{2}=\mathbf{E}\left[(\hat{Q}-Q)^{2}\right] \approx \hat{Q}^{2}\left(\frac{\sigma_{v}^{2}}{\hat{f}_{v}^{2}}-2 \rho_{t v} \frac{\sigma_{v}}{\hat{f}_{v}} \frac{\sigma_{t}}{\hat{f}_{t}}+\frac{\sigma_{t}^{2}}{\hat{f}_{t}^{2}}\right),
$$

where $\rho_{t v}$ is the correlation between the errors in the transverse and vertical profile coefficients. The error variance $\sigma_{\hat{Q}}^{2}$ is a good estimate of the mean squared error, as long as the modeled profiles are close to the instantaneous profiles $\left(\varepsilon_{t}<f_{t}, \varepsilon_{v}<f_{v}\right)$.

Thus, the error of the discharge estimate depends on two factors. First, on how well the velocity profiles are predicted. The better the model replicates the velocity profile, the better the discharge estimate will be. Second, on how large the magnitude of the measured velocity with respect to the cross section average is, because any error in the measurement or model is scaled up by the reciprocal of the velocity profile. This reduces the accuracy of predicted discharge, if the velocity is measured at a point where the velocity is low. This is the case near the banks and near the bed.

\subsubsection{Discharge prediction and error estimate for measurements along a range}

The discharge prediction can be improved by measuring the velocity at several points in the cross section. Velocity profilers in particular measure at several points that lie on one line. As most rivers are much wider than they are deep, velocity profilers are typically deployed so that their line of measurement spans horizontally across the river. The measurement points of HADCPs are spaced in discrete intervals, which makes the processing straightforward. In a first step, the discharge $\hat{Q}_{i}$ can be predicted for each individual measurement point. In a second step, the values of $Q_{i}$ can be arithmetically averaged into a single value $\hat{Q}_{a}$ :

$$
\hat{Q}_{a}=\frac{1}{\mathrm{~m}} \sum_{i=1}^{\mathrm{m}} \hat{Q}_{i}
$$

where $i$ is the index within the measured velocity profile and $M$ is the number of points at which the velocity is measured. Similar to the error of the 
discharge predicted from a single value (equation (2.7)), the error variance of the arithmetic average is

$$
\sigma_{\hat{Q}_{a}}^{2}=\mathbf{E}\left[\left(\hat{Q}_{a}-Q\right)^{2}\right] \approx \frac{Q_{a}^{2}}{\mathrm{~m}^{2}} \mathbf{E}\left[\left(\sum_{i=1}^{\mathrm{m}} \frac{\varepsilon_{v, i}}{\hat{f}_{v, i}}+\sum_{i=1}^{\mathrm{m}} \frac{\varepsilon_{t, i}}{\hat{f}_{t, i}}\right)^{2}\right] .
$$

The prediction error of the range-average is affected by the spatial correlation of the errors in the point estimates, and by the finite width of the cross section. The spatial correlation reduces the accuracy of the average of nearby samples. The finite width of the cross section increases the accuracy. In particular, if the velocity were measured across the entire cross section, then the uncertainty due to the transverse profile of the velocity would vanish. The actual number of measurement points along the range does not matter, as long as the distance between the points is small compared to the correlation length of the error.

To further simplify the error estimate for $\sigma_{\hat{Q}_{a}}$, we assume that the error variance of the predicted velocity profiles is constant across the profiling range, and that the correlation of the error decreases exponentially with increasing distance between two points. With this simplification, the error model is described by a first-order spatial autoregressive process (AR1):

$$
a_{t, l}=\mathbf{E}\left[\varepsilon_{t}(\xi) \varepsilon_{t}(\xi+l / w)\right]=\sigma_{t}^{2} \exp \left(-l / L_{t}\right),
$$

where $\mathrm{L}_{t}$ is the correlation length, $\mathrm{w}$ the cross section width, $l$ the distance between two points, and $\xi=N / w$ the normalized distance from the cross section center. If the velocity is measured in finite intervals $\Delta n=\Delta \xi w$ along the cross section, then the correlation length is related to the correlation coefficient as $\rho_{t}=\exp \left(-\Delta n / L_{t}\right)$.

The velocity profile coefficients $\hat{f}_{t}$ and $\hat{f}_{v}$ vary relatively little across the inner region of a cross section. In this region, the error variance of the range average is approximately

$$
\sigma_{\hat{Q}}^{2} \approx \hat{Q}^{2}\left(g_{v}^{2} \sigma_{v}^{2} \frac{1}{\hat{f}_{v}^{2}}+2 \rho_{v, t} g_{t v}^{2} \sigma_{v} \sigma_{t} \frac{1}{\hat{f}_{v} \hat{f}_{t}}+g_{t}^{2} \sigma_{t}^{2} \frac{1}{\hat{f}_{t}^{2}}\right) .
$$

The factors $g_{v}, g_{t}$, and $g_{v, t}$ account for the effects of spatial correlation and finite cross section width. The equations of these factors are given in appendix 2.9.1. The error estimate of equation (2.11) is strongly simplified, but it contains the most important factors that influence the predicted discharge.

The error of the discharge predicted from a single point is large at locations where the flow velocity is low. A single point measured close to the bank can 
thus deteriorate the arithmetic average over the entire profile. This can be avoided by predicting the discharge as $Q_{h}$, the product of the harmonic mean of the reciprocal velocity profile and the arithmetic mean of the measured velocity:

$$
\hat{Q}_{h}=\hat{A} \frac{1}{\sum_{i=1}^{\mathrm{m}} \hat{f}_{v, i} \hat{f}_{t, i}} \sum_{i=1}^{\mathrm{m}} \hat{u}_{i} .
$$

This estimate is identical to the index velocity method (IVM) with a variable coefficient. The sensitivity of the harmonic mean with respect to the transverse profile it is $\frac{\partial \mathcal{Q}_{h}}{\partial f_{t, i}}=\frac{-f_{v, i}}{\sum_{j=1}^{\mathrm{m}} f_{v, j} f_{t, j}} \mathcal{Q}_{h}$ and with respect to the vertical profile it is $\frac{\partial \mathcal{Q}_{h}}{\partial f_{v, i}}=\frac{-f_{t, i}}{\sum_{j=1}^{\mathrm{m}} f_{v, j} f_{t, j}} \mathcal{Q}_{h}$. The discharge predicted with the harmonic means is not sensitive to measurements close to the bank, as long as the profiling range reaches into the inner region of the cross section, where the profile coefficients $\hat{f}_{t, j}$ and $\hat{f}_{v, j}$ are above unity. If the velocity profile is constant along the profiling range, which approximately holds in the inner region of the cross section, then the prediction of the harmonic mean is identical to the arithmetic mean, and the error estimate equation (2.11) applies.

\subsubsection{Shape of the velocity profiles and sensitivity to perturbations}

Both the transverse and vertical profiles of the velocity can be approximated by simplifying the equations of fluid motion. This gives insight into how sensitive the velocity profiles are to changes in the flow conditions.

The transverse profile of the streamwise velocity can be predicted with a simplified form of the momentum equation (Shiono and Knight, 1989). When along-channel and across-channel slopes of the bed are gentle, then the depthaveraged velocity is approximately $\bar{u}=C \sqrt{h S}$ at a local point in the cross section. Where $h$ is the local depth, $C$ is the Chézy coefficient and $S$ the water surface slope. This is similar to Chézy's equation, which gives the cross section averaged velocity as

$$
U=C \sqrt{R_{h} S}
$$

where $R_{h}$ is the hydraulic radius. The transverse profile is consequently

$$
f_{t}=\sqrt{\frac{h}{R_{h}}} .
$$


The relative sensitivity of the transverse profile with respect to changes in the water level $z_{s}$ is

$$
\frac{R_{h}}{f_{t}} \frac{\partial f_{t}}{\partial z_{s}}=\frac{-1}{2} \frac{R_{h}-h}{h} .
$$

At places where the bed is not gently sloping, $f_{t}$ is more sensitive to changes in the water level than given by the linearization (equation (2.14)). In practice, the cross section geometry varies along the channel, and the bed level does not slope gently across the section, so that it cannot be determined from a simple equation. The transverse profile of the velocity is therefore often empirically determined by measurements with boat mounted vertical ADCPs (Hoitink et al., 2009).

The vertical profile of the streamwise velocity is close to logarithmic in open channels where the geometry gradually varies. The vertical profile of the velocity can be expressed by the log-law:

$$
u=\frac{u_{*}}{\kappa} \ln \left(\frac{h \eta}{z_{0}}\right)+u_{*} W,
$$

where $\kappa$ is the Kármán constant, $z_{0}$ is the roughness length, $u_{*}$ the shear velocity, and $h$ the water depth and $\eta=z / h$ the normalized distance above the bed. $W$ is the wake function that corrects for the systematic deviation of the velocity profile from a logarithmic shape (Coles, 1956). A rearrangement of the log-law and normalization by the depth-averaged velocity, obtained by integration of equation (2.16), gives the vertical profile:

$$
f_{v}=\frac{u}{\bar{u}}=\frac{\ln (\eta h)-\ln \left(z_{0}\right)+\frac{1}{\kappa} W(\eta h)}{\ln (h)-\ln \left(z_{0}\right)-1} .
$$

The roughness length is a model parameter that can be determined from reference measurements. The influence of the wake $W$ is small in wide channels with low along-channel water level gradients. When the wake is negligible, the sensitivity with respect to changes in the water level is

$$
\frac{h}{f_{v}} \frac{\partial f_{v}}{\partial z_{s}}=\frac{1}{\ln \left(z_{0}\right)-\ln (h)+1} .
$$

This is identical to the well known quantity $\frac{\sqrt{g}}{\kappa C}$. The vertical profile is furthermore sensitive to variations of the roughness length. In case of a negligible wake, this is:

$$
\frac{z_{0}}{f_{v}} \frac{\partial f_{v}}{\partial z_{0}}=\frac{\ln (\eta)+1}{\left(\ln (\eta)-\ln \left(z_{0}\right)\right)\left(\ln (h)-\ln \left(z_{0}\right)-1\right)}
$$




\subsection{Field sites}

We evaluate the performance of HADCPs at two separate discharge monitoring stations. The stations are located along the Kapuas river. The Kapuas is the largest river on the island of Borneo, with a catchment area of $9.910^{4} \mathrm{~km}^{2}$ (Hidayat et al., 2017). It flows into the Karimata Strait which separates Borneo from the island of Sumatra and mainland Asia. The Kapuas river is not yet restricted in its flow by dams or artificial levees. The stations are located at the Sanggau and Rasau municipalities, located 285 and $35 \mathrm{~km}$ upstream from the sea (Figure 2.1a). Discharge was continuously monitored from December 2013 to April 2015. The discharge is strongly influenced by the monsoon. During the monitoring period, the discharge ranged from $0.910^{3} \mathrm{~m}^{3} / \mathrm{s}$ to $9.510^{3} \mathrm{~m}^{3} / \mathrm{s}$ at Sanggau. These values are close to base flow and bankfull flow (Figure 2.2). Overbank flow rarely occurs at the gauging stations. At Sanggau, the hydraulic radius ranges between $14 \mathrm{~m}$ during high flow and $4 \mathrm{~m}$ during low flow. Between Sanggau and Rasau there are no large confluences, but three bifurcations (chapter 3). The latter divert part of the discharge to minor distributaries, so that the discharge at Rasau is on average $72 \%$ of that at Sanggau. At Rasau, the cross section has an average hydraulic radius of $16 \mathrm{~m}$. The daily averaged water level ranges seasonally only by $0.8 \mathrm{~m}$. The tide in the Kapuas is mainly diurnal. At Rasau, the spring tide ranges up to $1.75 \mathrm{~m}$. Reverse flow can occur when the discharge at Sanggau falls below $5,000 \mathrm{~m}^{3} / \mathrm{s}$, depending on the tidal range. Reverse flow was observed during $18 \%$ of the tidal cycles over the deployment period. The tide dissipates before reaching Sanggau during high and mean flow. During low flow, the tide ranges up to $0.6 \mathrm{~m}$. At Sanggau, the instruments were deployed in a large river bend with a radius of $1,500 \mathrm{~m}$. At this site, the river is $660 \mathrm{~m}$ wide during bankfull flow and recedes by $30 \mathrm{~m}$ from the top of both banks towards low flow. The river is slightly deeper in the outer bend and the bed level drops towards a pool situated further downstream (Figure 2.3a). In the inner bend, the river bed consists of sand and is covered by dunes. In the outer bend, the river bed consists of gravel and is not covered by dunes. At Rasau, the HADCP was deployed in a straight shallow reach between two bends. At this site, the Kapuas is $450 \mathrm{~m}$ wide and reaches a minimum bed level of $21 \mathrm{~m}$ below sea level in the left half of the cross section, with respect to the direction of the river flow (Figure 2.3b). The bed consists of fine sand (chapter 3). Dunes of considerable size develop only during periods of high river discharge, and then only in the right half. 

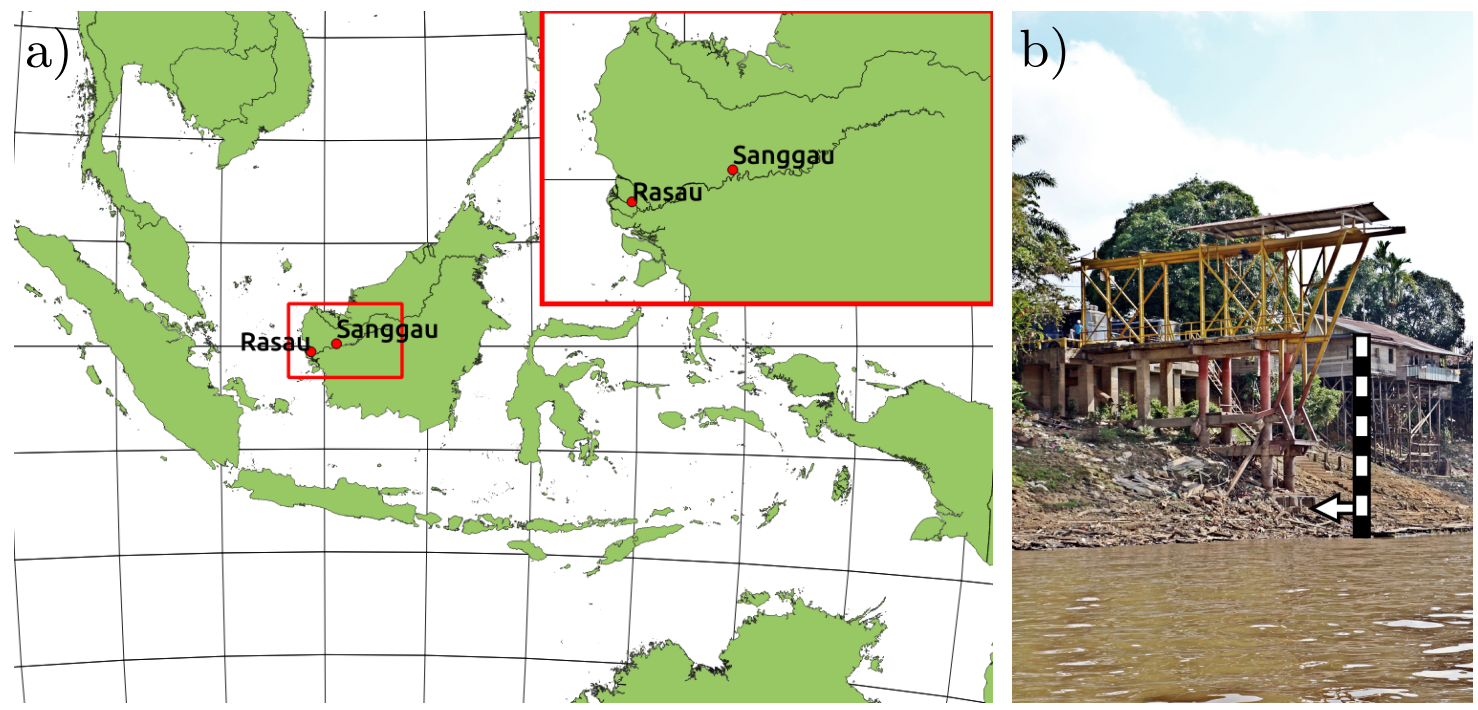

Figure 2.1: a) Location of the Kapuas catchment (rectangle) and of the two gauging stations (red dots in inset), Map based on GADM (Hijmans and et al., 2012) and Landsat (U. S. Geological Survey, 1972-2015) data; b) Jetty at Sanggau, where one of the HADCPs was deployed. The scale marks depth below bankfull water level in metres. The arrow indicates the HADCP position after redeployment. When the picture was taken the instrument was not submerged and did not measure.

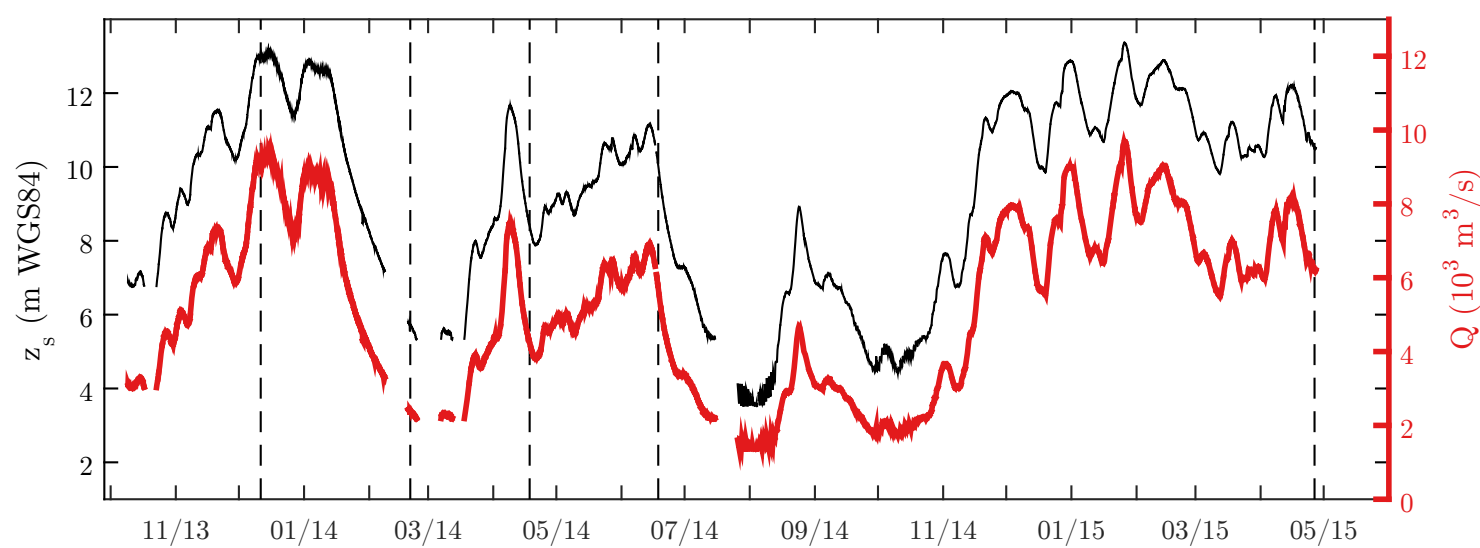

Figure 2.2: Water level and discharge monitored at Sanggau, vertical lines indicate days for which VADCP reference measurements are available 

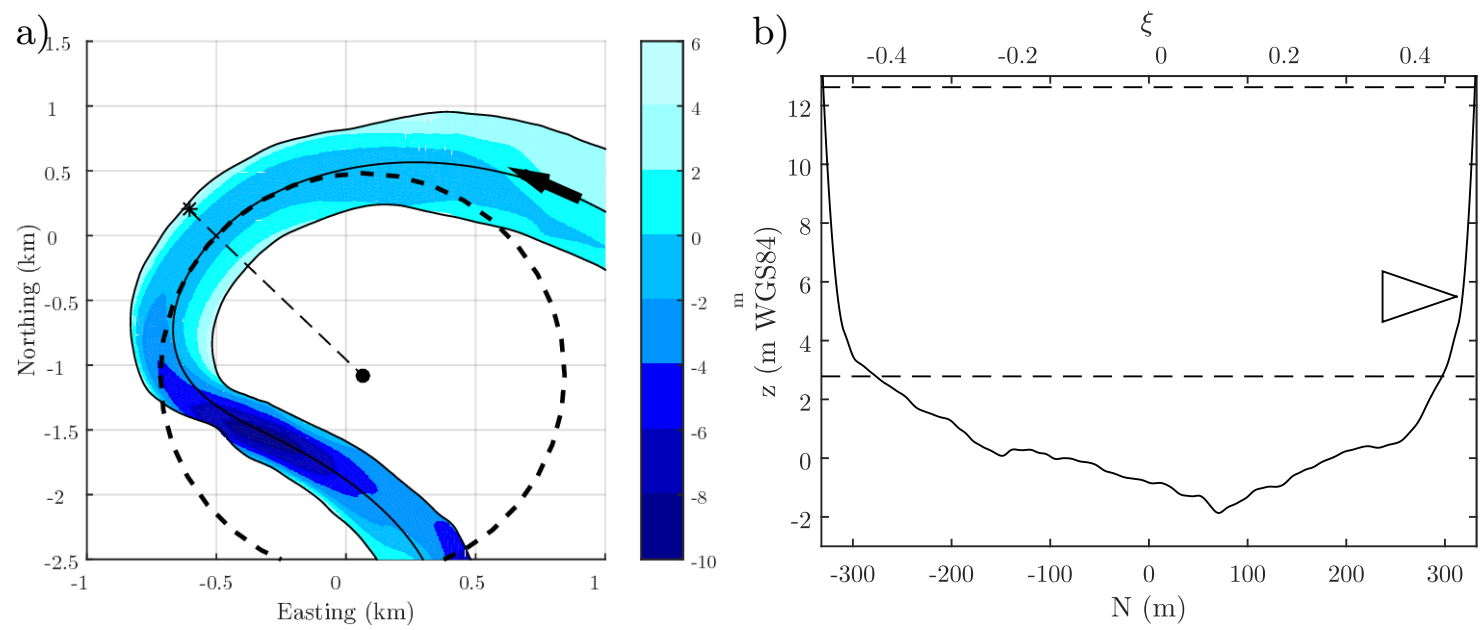

Figure 2.3: a) Bathymetry of the Kapuas at Sanggau and b) bed profile of the cross section, the dashed lines indicate maximum and minimum water levels, the triangle indicates the HADCP profiling range.

\subsubsection{Data acquisition}

At Sanggau, a $600 \mathrm{MHz}$ RDI Workhorse horizontal ADCP was deployed at a large jetty $17 \mathrm{~m}$ from the outer bank together with an air pressure compensated water level gauge of type Keller DCX22-AA. The profiling level is $9.4 \mathrm{~m}$ above the thalweg and $6.4 \mathrm{~m}$ below the top of the banks (Figure 2.4b). Both instruments fell dry during a low flow event in February 2014 and were redeployed to a $2.1 \mathrm{~m}$ lower level. The profiling axis is aligned with the shortest path between the banks. The effective profiling range is $65 \mathrm{~m}$ and thus spans across one tenth of the river width (Figure 2.3b). The velocity is measured every half hour. Each measurement lasts for 10 min during which the velocity is sampled in a $1 \mathrm{~s}$ interval and averaged. This protocol had previously been used by Hoitink et al. (2009) and Sassi et al. (2011b).

For Sanggau, five reference measurements are available. Those cover the entire seasonal variation of the hydrograph (Figure 2.2). The velocity was measured from a moving boat with a 1,200 kHz RDI VADCP and the position determined with a Vector Lite heading GPS. Each reference measurement consists of 8-20 crossings of the river to average out turbulent fluctuations. The individual crossings are displaced slightly up and downstream of the HADCP to reduce spatial variations over river dunes. The boat velocity ranges between 0.9 and $1.7 \mathrm{~m} / \mathrm{s}$. In addition, three bed level soundings are available in the period from 2012 to 2016 . The last sounding is vertically referenced with Terrastar satellite data. 
At Rasau, the HADCP was deployed likewise at a large jetty, about $23 \mathrm{~m}$ from the bank (Figure 2.4b). The profiling level is $4.6 \mathrm{~m}$ below sea level. The effective profiling range is $92 \mathrm{~m}$, and thus spans one quarter of the cross section. The instrument settings are the same as in Sanggau. At Rasau, seven VADCP reference measurements of varying duration are available. The measurements total to four diurnal cycles and include one complete diurnal cycle.
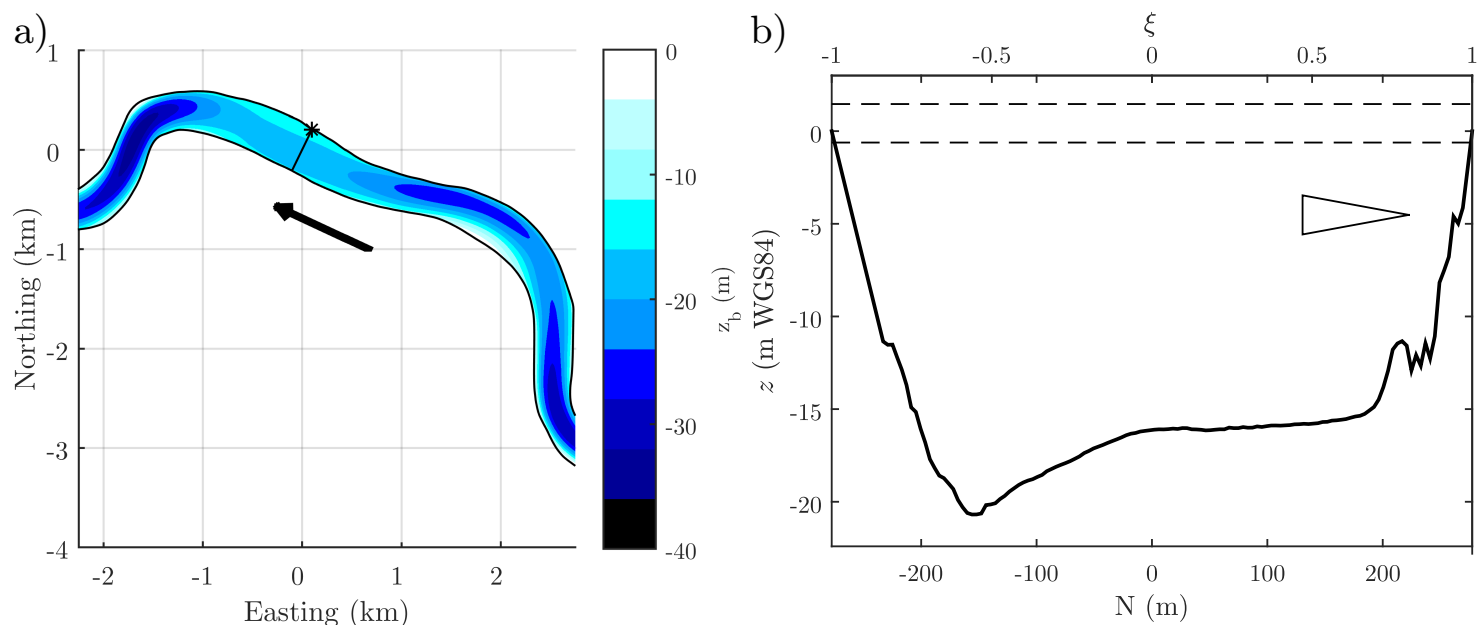

Figure 2.4: a) Bathymetry of the Kapuas at Rasau and b) bed profile of the cross section, the dashed lines indicate maximum and minimum water level, the triangle indicates the HADCP profiling range, the cross section is shallow and located in between two pools exceeding $50 \mathrm{~m}$ depth.

\subsection{Data processing}

\subsubsection{Determination of reference discharge and profiles}

We process the moving boat VADCP data with Matlab scripts using the opensource ADCP toolbox by Vermeulen et al. (2014b). In a first step, we preprocess the VADCP data. We determine the specific discharge by integrating the velocity over depth. We extrapolate the parts of the vertical profile near the surface and the bottom that are not measured by the VADCP, by applying noslip and no-shear boundary conditions, respectively. The vertical profile is fit to a re-parametrized form of the log-law (Wilkinson, 1983). We use a wake function as proposed by Granville (1976):

$$
u=a \ln (z)+b+c\left(2 \eta-3 \eta^{2}\right) .
$$

This allows us to determine the parameters $a, b$, andc with ordinary least-squares regression. The physical quantities follow from the regression parameters: the 
shear velocity $u_{*}$ as $\kappa a$, the roughness length $z_{0}$ as $\exp (-b / a)$, and the wake parameter as $c / a$. We define the velocity profile to be close to logarithmic if it has neither a local maximum nor an inflection point. This is the case as long as $-1 / 6 \mathrm{a}<\mathrm{c}<1 / 4 \mathrm{a}$. The roughness length and wake parameter can only be determined if the relative error of the shear velocity is smaller than unity. This is not the case for individual ensembles. The shear velocity therefore has to be smoothed before these two quantities are computed.

In a second step, we determine the transverse profiles of all flow parameters. At Sanggau, where the flow is stationary, we mesh the cross section into equally spaced elements of $1 \mathrm{~m}$ width. At Rasau, where the flow is modulated by tides, we also discretize the measurement period into equally spaced intervals of $30 \mathrm{~min}$. The VADCP measurements lack data close to the bank. The measurements lasting for an entire tidal cycle have also short gaps. We bin the velocity in discrete intervals over the cross section and extrapolate towards the banks with no slip boundary conditions. We fit the profiles of the bed level, depth-averaged flow velocity, specific discharge $(h \bar{u})$, and vertical profile parameters $a, b$, and $c$ individually. We determine discharge $Q$ by integrating the specific discharge across the section, and the normalized velocity profile $f_{t}$ as the ratio of the depth-averaged velocity $\bar{u}$ and cross section averaged flow velocity $(Q / A)$.

In a third step we determine the reference profile coefficients $\hat{f}_{t}, \hat{f}_{v}$, and $\ln \left(z_{0}\right)$. As the velocity profile is not defined for moments of slack water, the arithmetic time-average of the profile does not exist. We therefore determine the model profile as the weighted average of the reference profiles, so that the error of the estimated discharge (equation (2.9)) is minimized:

$$
\begin{aligned}
\hat{f}_{t, i} & =\sum_{k} w_{i} \hat{f}_{t, i, k}, \\
w_{i, k} & =\frac{Q_{k} A_{k} \bar{u}_{i, k}}{\sum_{k} Q_{k} A_{k} \bar{u}_{i, k}},
\end{aligned}
$$

where $k$ is the index of the reference profile and $i$ the index of the position within the cross section. The equations for variances and covariance are given in appendix 2.8.

In the case of Sanggau, where the water level range is large, we predict the transverse profile of the velocity empirically with a linear polynomial:

$$
\hat{f}_{t}=c_{t, 0}+c_{t, 1}\left(z_{s}-z_{r}\right),
$$

where $z_{s}$ is the water level and $z_{r}$ a reference level.

Finally, we estimate the HADCP performance, by the following procedure: 
1. Predict the velocity profile coefficients with a method of choice, for example empirically, as the weighted average of the reference measurements using equation (2.21a).

2. Compute the residual $\varepsilon_{t}=\hat{f}_{t, k}-\hat{f}_{t}$ of the predicted and measured profile coefficients.

3. Compute the root-mean-square error $\sigma_{t}$ of the residual using equation (2.24).

4. Compute the autocorrelation function $a_{t, i} / a_{t, 0}$ of the residual using equation (2.25).

5. Extract the correlation coefficient $\rho$ from the autocorrelation function; we propose a non-linear least-squares fit with weights $n-i$ and initial values $a_{t, 1} / a_{t, 0}$.

6. Upscale the mean square error to account for averaging over the sampling interval.

7. Estimate the error for an arbitrary profiling range using equation (2.30b).

\subsubsection{HADCP Discharge estimation}

We use the index velocity method (Le Coz et al., 2008; Levesque and Oberg, 2012) to predict the discharge from the HADCP velocity:

$$
Q_{H A D C P}=\left(\sum_{i=0}^{k} c_{i}\left(R_{h}\right)^{i}\right) A U_{I V M}+c_{A} A .
$$

$U_{I V M}$ is the index velocity, $A$ is the cross section area, $c_{i}$ are coefficients scaling the i-th power of the hydraulic radius $R_{h}$, and $k$ the number of terms used.

We do not use the arithmetic average along the HADCP profiling range as the index velocity $U_{I V M}$, but prescale it with the harmonic mean of the inverse velocity profile coefficients over the profiling range (equation (2.12)). At Rasau, we furthermore compensate the phase lag along the profile before averaging. We correct the phase for each species individually, as the phase lag is frequency dependent. With the pre-scaling, the theoretical values of the calibration coefficients for equation (2.23) are $c_{0}=1$ and $c_{i \neq 0}=0$.

\subsubsection{Rating curve discharge estimate}

Rating curves are functional relations between water level and discharge (Henderson, 1966). If the flow is well represented by a kinematic wave, then the stationary discharge is given by Chézy's equation (equation (2.13)). The bed 
slope $S$ is assumed equal to the water surface slope, which holds for uniform flow conditions. The water surface slope is higher during rising water level and lower during falling water level. This introduces a hysteresis into the stage-discharge relation. The hysteresis can be accounted for with Jones' formula, which corrects the velocity depending on the rate of change of the water surface level (Jones, 1916). Several extensions of Jones formula exist that correct for additional effects (Dottori et al., 2009). Rating curves perform well when the flow is uniform, such that the kinematic wave theory applies. Rating curves are not reliable at tidally influenced gauging stations. We therefore only compare the HADCP discharge estimate with a rating curve at Sanggau, the upstream gauging station.

\subsection{Evaluation of HADCP performance at two gauging stations}

\subsubsection{Transverse profile of the streamwise velocity}

At Sanggau, the transverse profile of the streamwise velocity $f_{t}$ has a slight transverse gradient $\frac{w}{U} \frac{\partial u}{\partial n}$ of about $15 \%$ during mid-flow, so that the velocity is higher in the outer bend than in the inner bend (Figure 2.5a, solid black). The velocity thus increases with the flow depth within the cross section (compare Figure 2.5a with Figure 2.3). The transverse gradient changes systematically with stage, so that it is larger at low-flow than at high flow (Figure 2.5a). The transverse profile is well predicted by a linear function (equation (2.22)). The linear prediction has a relative error $\frac{1}{f_{t}} \sigma_{t}$ of $2.6 \%$ in the inner region of the cross section (Figure 2.5a). The error is correlated over a distance of $7 \mathrm{~m}$ (Figure 2.6a). Due to the systematic change over the hydrograph, the average of the reference profiles is not a good predictor of the instantaneous profiles. It has a larger error and longer correlation length than the linearly predicted profile. Towards the banks, the velocity profile has a large transverse gradient and drops to zero, and the relative change of the velocity profile is large. This causes the relative error to become very large near the bank. The cross section averaged velocity is reached at a distance of $55 \mathrm{~m}$ away from bank at high flow, but only after $95 \mathrm{~m}$ at low flow. The effective profiling range of the HADCP thus does not reach beyond the region of low velocity near the bank.

At Rasau, the transverse profile is nearly uniform in the central region of the cross section and sharply drops towards either bank (Figure 2.5b). This is similar to Sanggau. It has a small transverse gradient and decreases from the bank where the HADCP is installed to the opposite bank. Thus, the velocity decreases with the flow depth (compare Figure 2.5b with Figure 2.4). The 
velocity profile does not systematically change between the reference measurements, which can be explained by the relatively small water level range with respect to the depth. We therefore model the profile as stationary in time. The stationary profile has a root-mean-square error of $3.9 \%$ in the inner region (Figure 2.5b). The error is correlated over a length of $47 \mathrm{~m}$ (Figure 2.6b). The near-bank region with low flow velocity reaches $90 \mathrm{~m}$ into the cross section at the bank of the HADCP and $45 \mathrm{~m}$ into the cross section on the opposite bank. Towards either bank, the error becomes arbitrarily large, as at Sanggau. The effective profiling range of the HADCP does not reach far beyond the near-bank region.
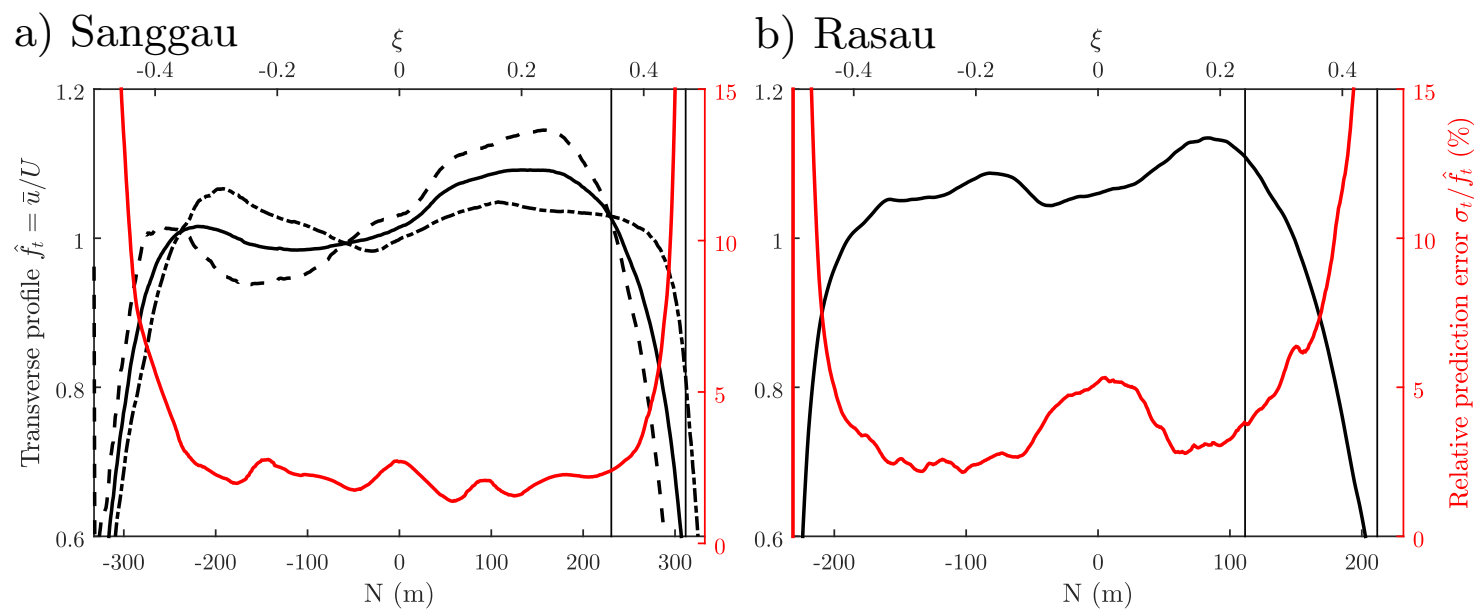

Figure 2.5: Normalized transverse profiles $\hat{f}_{t}$ of the streamwise velocity (black) at a) Sanggau and b) Rasau; at Sanggau, the profile systematically varies between low flow (dashed) and high flow (dash-dotted). The relative prediction error of the velocity profile (red), diverges throughout the outer region towards the bank. The HADCP profiling range (vertical lines) is limited to the near-bank region at both sites, which compromises the discharge estimate.

\subsubsection{Vertical profile of the streamwise velocity}

At Sanggau, the vertical profile of the streamwise velocity is close to logarithmic. The profile tends to have a submerged maximum in the inner bend and an inflection point in the outer bend, but the effect of the wake is overall negligible. During mean flow, the vertical profile coefficient at the profiling depth $\hat{f}_{v}$ reaches a maximum of 1.1 at the channel centre and slightly decreases towards both sides (Figure 2.7a). This is consistent with the bed profile, which causes the instrument position to be relatively high up in the water column 
a) Sanggau

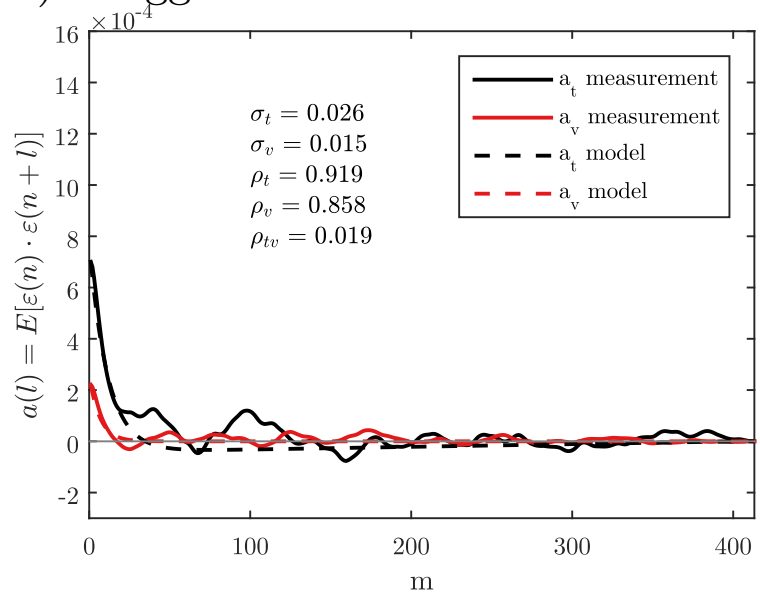

b) Rasau

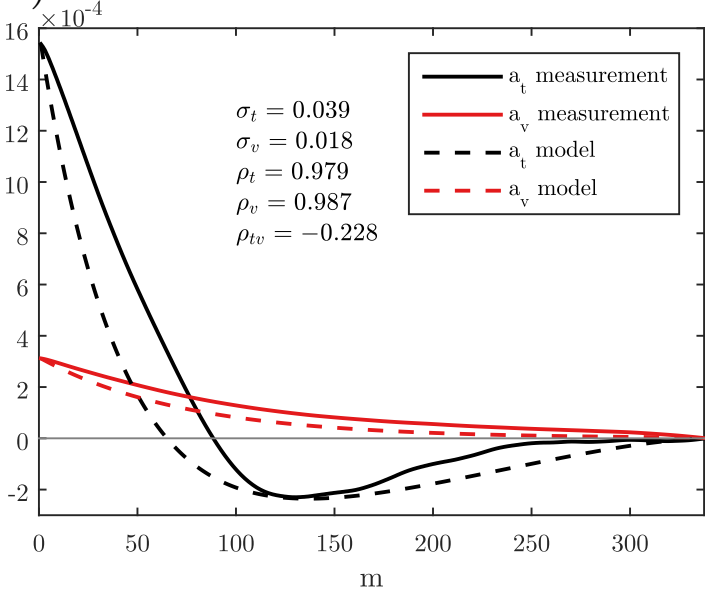

Figure 2.6: Auto-covariance of the prediction error of the velocity profiles in the inner region of the cross section at a) Sanggau and b) Rasau; the prediction error of the transverse profile (black) is larger than that of the vertical profile (red), but correlates over a shorter distance, At Sanggau, both the magnitude and the correlation of the error due to the transverse profile are much larger when the profile were modeled as stationary.

near the channel centre. Beyond the near-bank region, the profile value is $10 \%$ higher during low flow than during high flow. This is consistent with the relative position of the instrument within the water column which changes with the hydrograph. A prediction of the vertical profile coefficients with the log-law (equation (2.16)) has a root-mean-square error of 1.5\%. The error is only correlated over a short distance $L_{v}=6 \mathrm{~m}$ (Figure 2.6a). As for the transverse profile, a prediction with the average of the reference profiles has a much larger prediction error, and is also correlated over a much longer distance.

At Rasau, the vertical profile has a pronounced velocity dip, so that the maximum occurs below the surface. The wake is strongest near the bank, but does not vanish towards the centre of the cross section. The wake parameter $c / a$ has an average value of 0.48 . The vertical profile coefficient $\hat{f}_{v}$ is almost constant over the cross section and has an average value of 1.1 (Figure 2.7b). The profile does not change systematically with the river discharge. This can be explained by the small variation of the water level with respect to depth. A prediction with a stationary profile has a root-mean-square error of only $1.8 \%$ (Figure 2.7). The error in the vertical profile coefficient is correlated over $74 \mathrm{~m}$ (Figure 2.6b). A prediction of the profile coefficient $\hat{f}_{v}$ from the water level is not better. The logarithmic shape of the velocity profile breaks down when the 
discharge drops below 2,000 $\mathrm{m}^{2} / \mathrm{s}$ around slack water. Near slack water, the relative error becomes very large, however, the absolute error remains low.

The vertical profile coefficient $\hat{f}_{v}$ does not only change with the water level but also change with the roughness length $z_{0}$, according to the log-law (equation (2.16)). An unpredicted change of the roughness introduces an error to $\hat{f}_{v}$ according to equation (2.19). The sensitivity is high in shallow water and above rough beds but does not exceed a few percent in practice (Figure $2.7 \mathrm{~b}$ ). At our field sites, the sensitivity with respect to relative deviations of $z_{0}$ does not exceed 0.02 and the relative root-mean-square deviation $\sigma_{z_{0}} / z_{0}$ is about 0.9 , so that the error of $f_{v}$ does not exceed $1.8 \%$. At both field sites, the roughness increases systematically with the discharge (Figure 2.8). The measured roughness matches well with the prediction from bed form size and bed material (van Rijn, 1984a). However, a prediction of the roughness length with stage did not considerably improve the prediction at either site. Variation of the vertical profile due to migrating bedforms and phase of the tidal cycle have to be considered to increase the accuracy of the prediction.

a) Sanggau

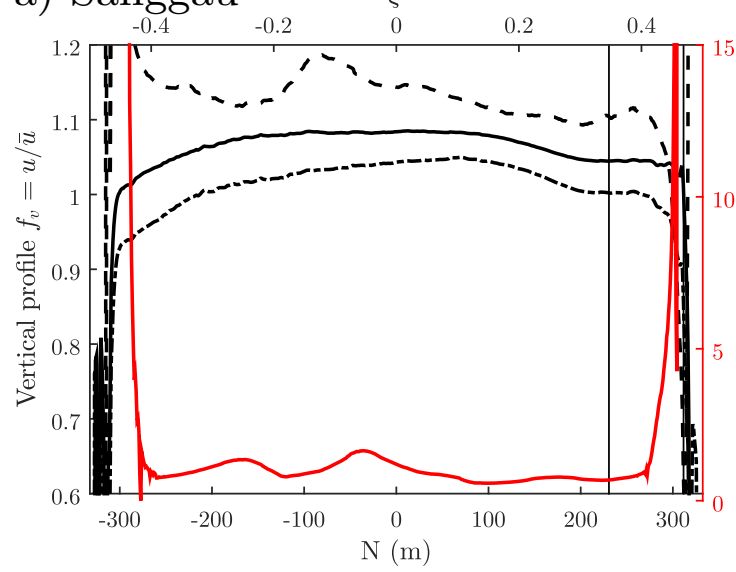

b) Rasau

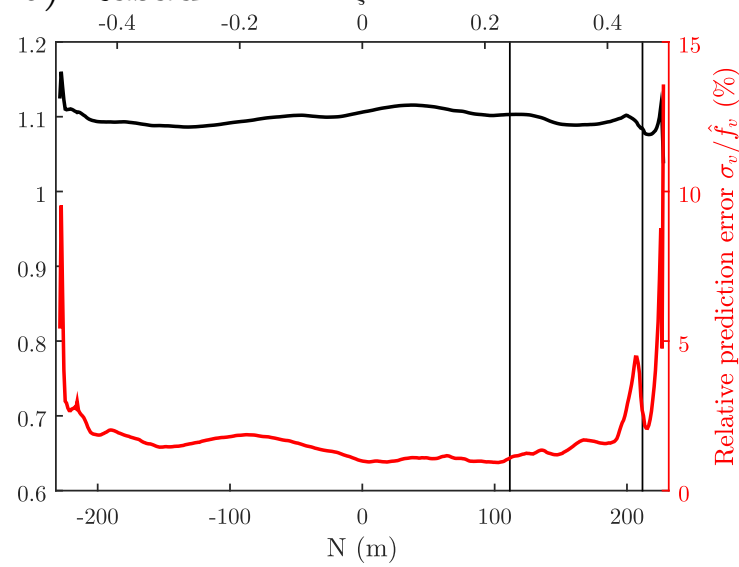

Figure 2.7: Coefficients of the normalized vertical profile of the streamwise velocity $\hat{f}_{v}$ at instrument depth (black) and its relative prediction error (red); a) Sanggau, b) Rasau; at Sanggau, the profile coefficient has a higher value at the instrument depth during low flow (dashed) than during high flow (dashdotted).

\subsubsection{Combined effect of transverse and vertical profiles}

At the two field sites the transverse profile coefficient coefficient $\hat{f}_{t}$ is less reliably predicted than the vertical profile coefficient $\hat{f}_{v}$ (c.f. Figure 2.5 and 2.7). The prediction error of the transverse profile coefficient is almost twice 
as large. This causes the contribution of the uncertainty in the vertical profile to the total error to be marginal, because the squares of the errors add. The cross correlation $\rho_{t v}$ between the error of the transverse and vertical profile coefficients is weak at both field sites, with values of 0.02 at Sanggau and 0.23 at Rasau, so that the contribution of the covariance between the profiles to the total error is also negligible.

Figure 2.9 shows the decrease of the prediction error with increasing profiling range along the inner region of the cross section. The error drops more rapidly at Sanggau (a) than at Rasau (b), because the error is correlated at this station over a shorter distance. The uncertainty in the transverse profile coefficient (black) dominates the total error (green) for short and intermediate profiling ranges. The uncertainty of the vertical profile coefficient (red) becomes only relevant when the profiling range extends almost across the entire river width.
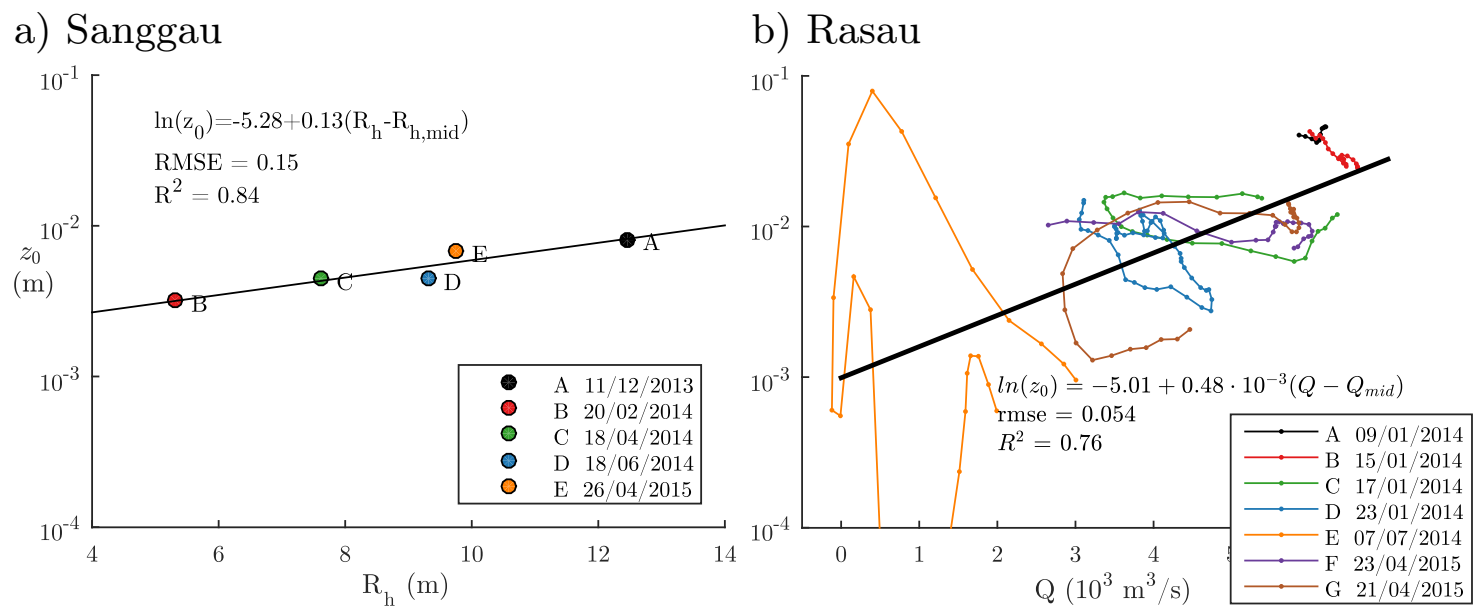

Figure 2.8: Roughness length at a) Sanggau and b) Rasau; at both stations, roughness increases with the river discharge. At Rasau, the bed is smoother during low and mean flow than at Sanggau. At Rasau, the roughness does slightly vary with the discharge within a single tidal cycle. Around slack water, below $2000 \mathrm{~m}^{3} / \mathrm{s}$, the vertical profile is far from logarithmic, so that the roughness length is not well defined.

\subsubsection{HADCP performance at Sanggau}

At Sanggau, the instrument level and alignment changed during the deployment. We therefore split the time series into three periods for which the instrument position remained constant. Due to the splitting, the HADCP was not directly calibrated against VADCP reference measurements, but against 
a) Sanggau

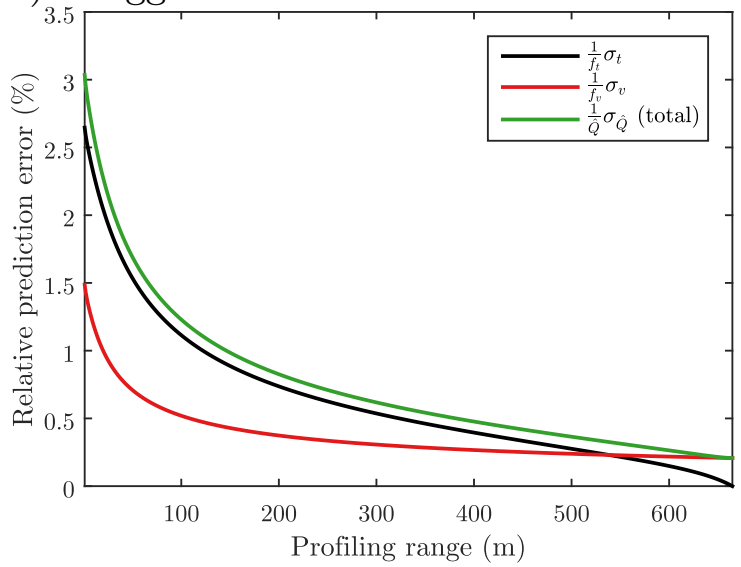

b) Rasau

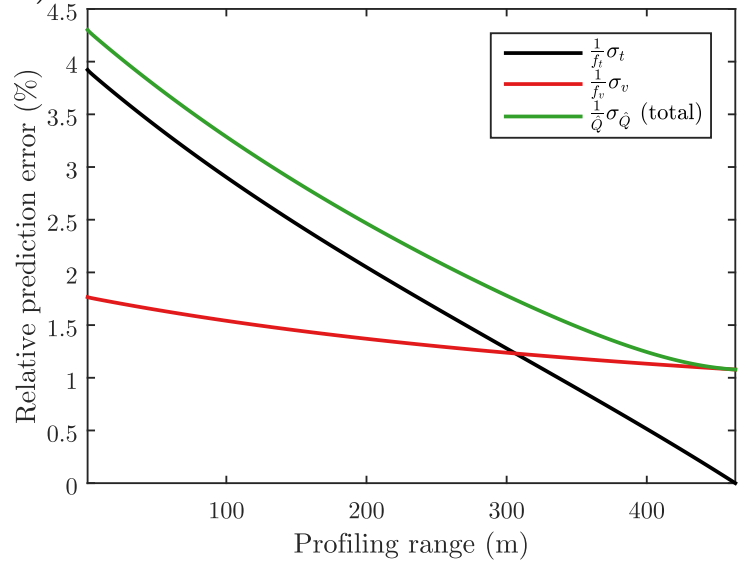

Figure 2.9: Relative error of the discharge prediction at Sanggau (a) and Rasau (b); for Sanggau, the velocity profile coefficient is predicted from the water level, at Rasau it is kept constant over time; valid for measurements in the inner region of the cross section where $f_{t}>1$; at both stations the error is dominated by uncertainty of the transverse profile coefficient $\sigma_{t}$, the error of vertical profile coefficient $\sigma_{v}$ is only relevant when the profiling spans more than half of the cross section

the rating curve. The discharge at Sanggau is reliably predicted by a simple power-law $Q_{R C}=c_{r 0} A R^{c_{r 1}}$ (Figure 2.10). We estimate the error by the residual $\varepsilon_{Q}=Q_{H A D C P}-Q_{R C}$. We predict the HADCP discharge several times with models of increasing complexity (equation (2.23)). For comparison, we predict the discharge solely from the cross section area while keeping the velocity constant. This reference overestimates the discharge at low flow and underestimates it at high flow, but the root mean square error is only $7 \%$ of the peak discharge (A in Figure 2.11). A prediction with the index velocity method with one term $\left(c_{0} A U_{I V M}\right)$ has a root-mean-square error of $3.8 \%$. The HADCP measurements thus only explain about half the variance of the flow velocity explained by the rating curve.

We introduce the term $A \frac{\partial R_{h}}{\partial t}$ to the rating curve to account for the stagedischarge hysteresis. However, only a negligible fraction of the velocity variation is caused by this term. A second term $\left(c_{1} A U_{I V M} R_{v}\right)$ in the HADCP prediction improves the discharge estimate to an error of less than $1 \%$. This confirms that the variation of the velocity profile with the water level indeed compromises the accuracy of the HADCP discharge prediction. Higher order terms do not improve the prediction further. The discharge predicted with the HADCP and the rating curve is in a reasonable agreement with each other, and the difference is close to the error estimated from the VADCP profile, which is $1.5 \%$ 

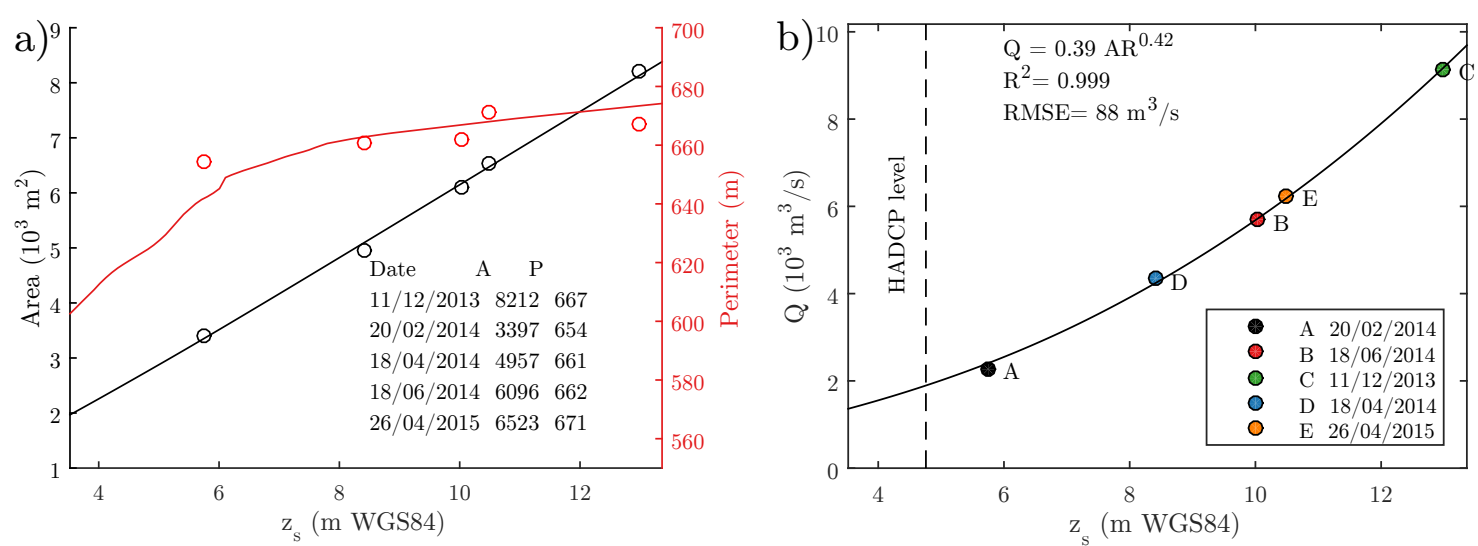

Figure 2.10: Cross-section area and wetted perimeter (a) as well as discharge rating curve (b) at Sanggau

at the full HADCP profiling range (Fig 2.9).
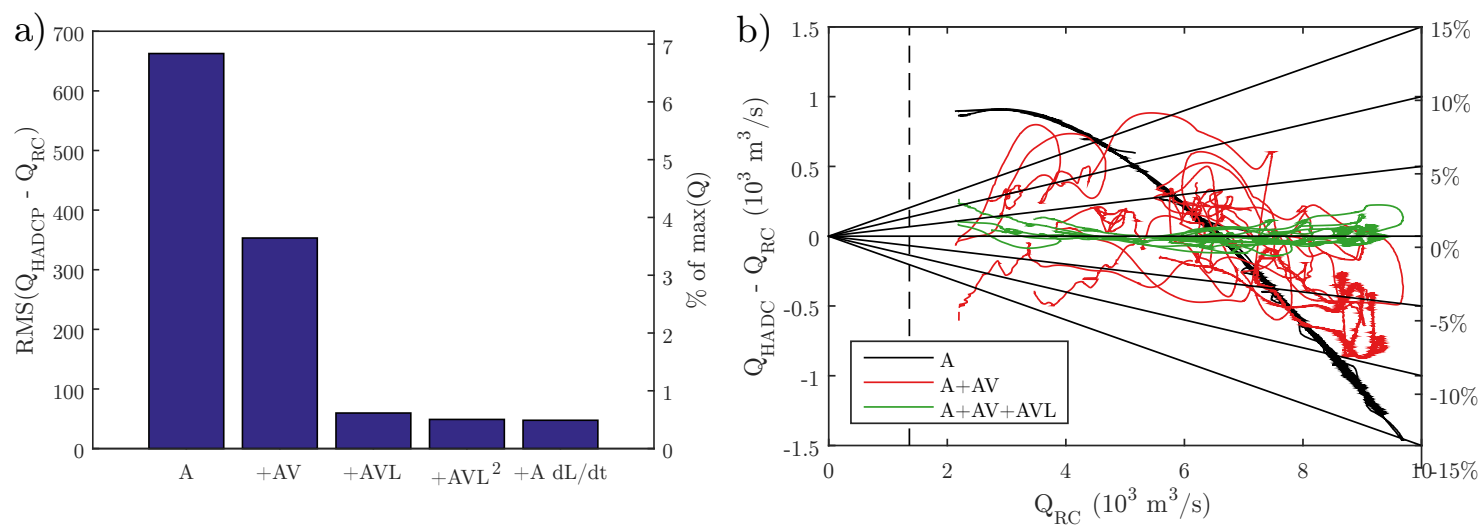

Figure 2.11: a) RMS-difference and b) difference between the rating curve and HADCP discharge estimates for increasingly complex HADCP discharge prediction.

\subsubsection{HADCP performance at Rasau}

Rasau is an appropriate site for an HADCP deployment, as the small water level range and the tide cause a rating curve to be unreliable. Here we directly compare the HADCP and VADCP discharge during the reference measurements (Figure 2.12). The root-mean-square deviation between the discharge determined with both methods is $4.3 \%$ of the peak discharge, and thus in agreement with the error estimated from the VADCP velocity profile (2.9a). 
The velocity along the HADCP profile drops exponentially towards the banks (Figure 2.13a), which is consistent with the VADCP measurement (Figure 2.5a) and expected from the theory (section 2.2.4). The magnitude of the velocity close to the instrument is only half as large as that of the cross section averaged velocity. The HADCP profiles up to $150 \mathrm{~m}$, but the measurement is reliable only up to $92 \mathrm{~m}$ from the instrument. During periods of reverse flow, salinity intrusion increased the attenuation and reduced the effective profiling range further. The velocity near the bank is also leading the velocity in the channel centre by about $1 \mathrm{~h}$ (Figure 2.13b). A phase lead near the bank is typical for tidal channels and has been corrected for at other HADCP deployments (Hoitink et al., 2009). At Rasau, both the velocity profile and phase lead differ considerably between the individual frequency components of the tide. We therefore correct for asynchronous velocity variation over the cross section by considering tidal species individually. The profiles of overtides vary more strongly over time and are less reliably monitored (Figure 2.13c). Species beyond the fifth-diurnal overtide cannot be reliably resolved with the deployment.

Variation of the velocity profile for different ratios of river and tidal velocity amplitudes are probably the main reason why the root-mean-square error of the HADCP discharge at Rasau is higher than at Sanggau. In particular, the profile strongly differs between seaward and reverse flow.

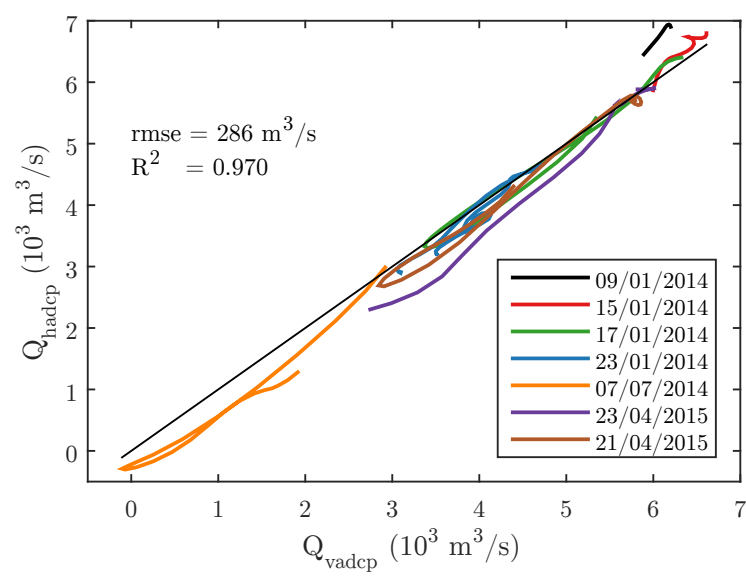

Figure 2.12: HADCP and VADCP discharge at Rasau during reference measurements. 

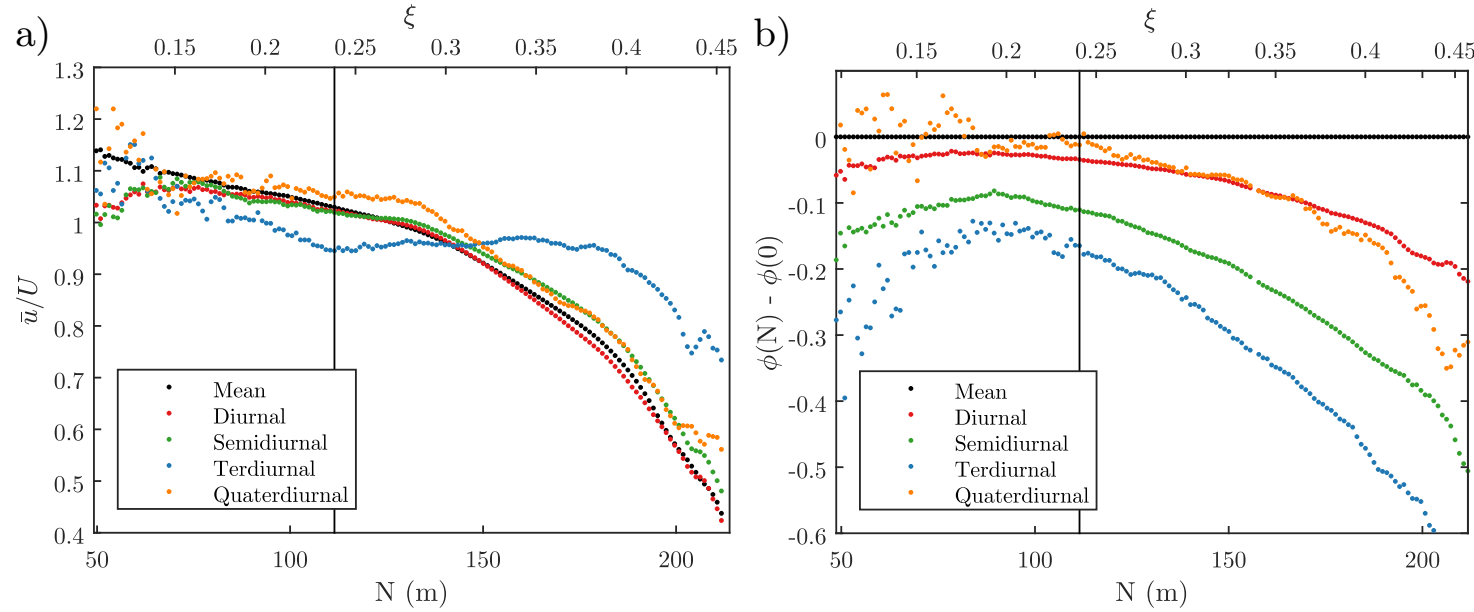

Figure 2.13: a) Velocity profile and b) phase shift $\Delta \phi$ as measured by the HADCP at Rasau; vertical lines indicate the effective range of the HADCP; both phase shift and the velocity profile differ between the tidal species.

\subsection{Discussion}

\subsubsection{Sources of error and limits of accuracy}

An order of magnitude analysis of the sensitivity of the velocity profiles (equations (2.15) and (2.18)) reveals that the sensitivity with respect to changes in the water level is large, in particular at points in the cross section where the water depth is much smaller than the hydraulic radius. The velocity profile is hence much more sensitive to perturbations near the banks, where the water is shallow. Systematic changes of the velocity profile with the water level therefore have to be corrected at stations where the water level range is large. This is in agreement with the observations at our field sites. At Sanggau, the water level range is large, and both the vertical and the transverse profile coefficients differ by more than $10 \%$ between low and high flow, even in the central region of the cross section. At Rasau, the water level range is small, and the velocity profiles do not strongly vary between low and high flow. Changes of the vertical profile can be reliably predicted with the log-law, but it is more difficult to predict the transverse profile. Extrapolation beyond the HADCP profiling range is hence the main source of error in the predicted discharge. The vertical profile is much less sensitive to changes of bed roughness (equation (2.19)) than to changes of the water level. This is because the sensitivity is zero when the velocity is measured at a relative depth of $\eta=0.4$, and remains small as long as the velocity is measured above this point.

The error of the discharge predicted from different measurement points 
across the section is correlated in space. The effective sample size of an HADCP measurement is therefore much lower than the number of measurement points along its range. A correction for changes of the velocity profile coefficients improves the discharge estimate twofold, both by reducing the prediction error for point measurements as well as by reducing the spatial correlation of the error (Figure 2.14b). Statistically, near-bank velocity measurements are less reliable indicators for the discharge than measurements in the inner region of the cross section, even if the velocity profile is uniform (Figure 2.14).
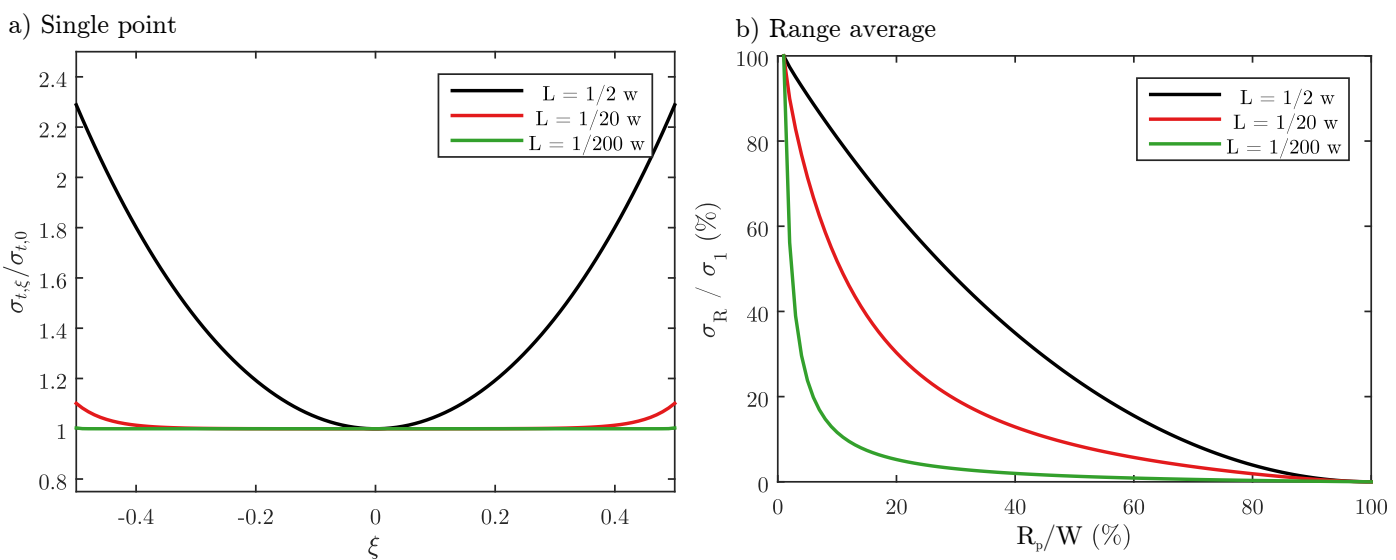

Figure 2.14: Standard error as given by the statistical model for a) a single point and b) range-averaged velocity measurement with respect to the cross section average, depending on the correlation length $L$, in case of a uniform transverse velocity profile (c.f. equations (2.28) and (2.30b)); both single point measurements (a) and range-averages (b) are more reliable, when the spatial correlation is lower.

\subsubsection{Optimal HADCP deployment}

The profiling range of HADCPs matters, as the prediction error decreases with increasing profiling range (Figure 2.14b). In an ideal case, the profiling range reaches across the entire river width, so that the variation of the transverse profile of the velocity does not deteriorate the prediction. If the range does not reach across the entire channel, then the location of the deployment affects the performance. As the variation of the velocity profile depends on the cross section geometry, it is advisable to deploy the instrument at a place where the variation is low. Because velocity measurements near the bank are less reliable, the profiling range should reach beyond the point where the depthaveraged velocity exceeds the cross section average. This roughly coincides 
with the point in the cross section, where the local depth equals the hydraulic radius, if the banks are sloping. The inner region retreats towards the channel centre during low flow. Instruments with a short profiling range should therefore be installed so that they monitor in the inner region of the cross section, for example at a bridge pillar rather than on a short jetty. Bridge pillars also allow for deployments at a sufficiently low level, so that the instrument can also measure at low flow.

For range-limited deployments, the variation of the vertical profile is less important, as it is usually well-predicted by the log-law. The deployment can nonetheless be optimized by placing the instrument at a distance of 0.4 times water depth above the bottom. At this location, the profile is insensitive to changes of the roughness length. Similar to the ideal points in the transverse profile, the local velocity equals depth-averaged velocity at this point. This is also the position recommended in the literature (Boiten, 2000) for single point measurements. The absolute location depends on the water level, so that measurements at low flow are more sensitive to perturbations of the profile. In cases of very shallow cross sections, a mechanized deployment moving the instrument along the vertical can be considered (Vougioukas et al., 2011).

\subsubsection{Experiences from field sites}

The observations at both field sites support the idea that the variation of the transverse profile of the streamwise velocity is a major source of error for discharge monitoring with range-limited HADCPs. At both field sites, the transverse profile of the velocity has a region of decreasing velocity and increasing variation towards the channel bank. At both field sites, the prediction of the transverse profile is less reliable than that of the vertical profile, although the causes of the profile variation differ between the field sites. At Sanggau, the variation of the profile depends on the water level and can be reliably predicted, whereas at Rasau, profile and phase shifts differ among the frequency components of the tide.

Discharge was previously monitored with a comparable HADCP at cross sections of $400 \mathrm{~m}$ width, in the tidal rivers of the Mahakam (Sassi et al., 2011b) and the Berau (Hoitink et al., 2009), as well as at a backwater affected site with a $250 \mathrm{~m}$ wide cross section with large water level range in the Mahakam (Hidayat et al., 2011). The size of these cross sections is probably at the upper limit where an instrument with a $75 \mathrm{~m}$ range (600 kHz sound frequency) is useful. At a cross section exceeding $500 \mathrm{~m}$ in width, an instrument with longer range, for example a $300 \mathrm{kHz}$ instead of a $600 \mathrm{kHz}$ ADCP, is a better alternative. 
The velocity profile can be predicted with a numerical model. A model that neglects along-channel variations of the cross section geometry was successfully applied by Nihei and Kimizu (2008) to predict discharge from HADCP measurements. We found that the velocity profiles are more readily predicted with a simple regression model. Secondary flow in river bends is known to influence the velocity profile (Blanckaert and De Vriend, 2004; Vermeulen et al., 2015) and may explain part of the variation. At Sanggau, the transverse profile also varied considerably due to migrating bed-forms. The instantaneous geometry of bed-forms is not predictable. Profile variation by bed-forms can thus only be removed by either averaging over timescales exceeding that of the dune migration, or averaging over long ranges that exceed the typical bed-form width. The bed at Sanggau has a relatively large local bed slope $\left(\approx 10^{-3}\right)$, which is an order of magnitude larger than the surface slope. As the Kapuas is meandering, the bed slope exceeds the surface slope by 1-2 orders of magnitude almost everywhere (chapter 3). These gradients strongly influence the velocity profile (Yang et al., 2006). At Rasau, the velocity is lower in the deeper part than in the shallower part of the cross section. Non-uniformity and unsteadiness of the flow can therefore have a considerable influence on the velocity profile.

\subsubsection{Comparison to rating curve discharge prediction}

An HADCP deployment and calibration is expensive and labour intensive. It is therefore only justified at gauging stations where conventional methods, such as rating curves, are not sufficiently reliable. Rating curves are susceptible to rapid changes of the water level, which often occur in small rivers. For example, the relative error of discharge predicted by rating curves exceeds $26 \%$ at some places along the Po river (Di Baldassarre and Montanari, 2009). In large rivers, the water level changes slowly in time, because precipitation does not occur simultaneously across the catchment and flood peaks diffuse. Rating curves can therefore be expected to be more reliable for reaches of large rivers that are not affected by backwater or tides.

At Sanggau, a stationary rating curve fits the reference measurements well, as there is an unambiguous relationship between flow velocity and water level (Figure 2.10). The rate of change of the water level at Sanggau is small and has a standard deviation of $0.2 \mathrm{~m} / \mathrm{d}$. The stage-discharge hysteresis thus introduces a relative error of less than $1 \%$ to the discharge estimate.

The short profiling range and strong variation of the velocity profile near the bank where the HADCP was deployed prevented the HADCP measurement 
to be reliable enough to correct for the relatively small stage-discharge hysteresis. The prediction of discharge from HADCP measurements also requires a correction for the systematic change of the velocity profile, even in steady flow conditions. Such a correction is not required for rating curves.

At low flows, the tide can intrude far and modulates the discharge even at upstream stations, as is the case in Sanggau. River-tide interaction also modulate the discharge over the spring-neap cycle (Buschman et al., 2009; Matte et al., 2014). Velocity measurements are therefore of particular interest at low flow. However, along natural rivers it can be difficult to deploy an HADCP low enough to measure during low flows, and a rating curve may be the only feasible option to monitor the discharge during these times.

\subsection{Conclusion}

This paper presents a generic theory to evaluate the performance of discharge monitoring approaches based on range-limited velocity profilers, such as HADCPs. In general, the accuracy depends on how much the ratio between the flow velocity in the measured part of the cross section and the cross section averaged velocity vary. This ratio can depend on the water level, even when the flow is nearly steady. The velocity profile varies strongly near the river banks. Therefore, the profiling range has to extend into the inner region of the cross section where the depth-averaged velocity reaches the cross section average. This requires a long profiling range wherever the banks are gently sloping and the water level range is large. Alternatively, the instrument can be deployed closer to the channel centre, for example at a bridge pillar. The accuracy of the discharge estimates is only weakly dependent on the vertical profile of the velocity, and therefore can be installed close to the bed. This allows to continue gauging during low-flow conditions.

We apply our theory to evaluate alternative approaches to gauging the discharge of the Kapuas, which is the largest river in Indonesia. The Kapuas catchment has remained ungauged so far, and we established for the first time a continuous discharge time series for a duration for 19 months. An HADCP was deployed to collect continuous velocity measurements. Mainly due to the limited profiling range, the discharge estimation method using the HADCP data did not outperform a basic rating curve. This example highlights that the range of an HADCP has to extend beyond the region influenced by the banks, covering at least part of the central section where the local flow velocity exceeds the cross section averaged velocity. 


\section{Appendix}

\subsection{Statistics of the transverse profile of the streamwise velocity}

If the coefficients of the transverse profile of the streamwise velocity $\hat{f}_{t}$ are determined as the weighted average as in equation (2.21a), then the variance and auto-covariance of the weighted residual are

$$
\begin{aligned}
\sigma_{t, k}^{2} & =\frac{\mathrm{n}}{\mathrm{n}-1} \frac{1}{\sum_{k} w_{k}^{2}} \sum_{k} w_{k}^{2}\left(\hat{f}_{t, k, i}-\hat{f}_{t, i}\right)^{2}, \\
a_{k,|i-j|} & =\frac{\mathrm{n}}{\mathrm{n}-1} \frac{1}{\sum_{k} w_{i, k} w_{j, k}} \sum_{k} w_{k, i} w_{k, j}\left(\hat{f}_{t, k, i}-\hat{f}_{i}\right)\left(\hat{f}_{t, k, i}-\hat{f}_{i}\right),
\end{aligned}
$$

where $\mathrm{n}$ is the effective sample size. In practice, this is the number of reference profiles available at distinct points of the hydrograph in case of stationary flow, or the number of distinct tidal cycles in the case of tidal flow. These estimates are valid if the residuals of the reference profiles are mutually independent. This is the case when the predicted velocity profiles are free of systematic error. In the case that the profile is predicted with a polynomial model (equation (2.22)), $w_{k}$ are the weights of the least-squares regression. Note that the weighted average remains valid and optimal, even if in parts of the cross section the bed falls dry, or slack water occurs.

\subsection{Error variance of a spatially correlated finite population}

To determine the error variance of the HADCP discharge estimate, the cross section is discretized in equally spaced intervals. Together, these intervals represent a finite population. The HADCP samples in its profiling range a subset of the population. With increasing profiling range, errors in the predicted velocity profile cancel each other during averaging. The error variance can be estimated by equation (2.9), but it does not immediately reveal the effect of range-averaging. The error variance is therefore analyzed based on a simplified equation (2.11) that represents the case of a uniform transverse profile of the velocity. In this equation, the factors $g_{t}, g_{v}$, and $g_{t, v}$ account for spatial correlation of the errors of the estimated profile coefficients $\hat{f}_{v}$ and $\hat{f}_{t}$, as well as the finite width of the cross section. To determine factors $g_{t}$ and $g_{v}$, at first a univariate autocorrelated series of finite length is studied. 


\subsubsection{Univariate case}

Consider the finite univariate process

$$
\begin{aligned}
\varepsilon_{1} & =\sigma \tilde{\varepsilon}_{1}, \\
\varepsilon_{i} & =\rho \varepsilon_{i-1}+\sqrt{1-\rho^{2}} \sigma \tilde{\varepsilon}_{i}, i=2, \ldots, \mathrm{n},
\end{aligned}
$$

where the $\tilde{\varepsilon}_{i}$ are mutually independent and identically normally distributed. Consider one realization of the process, forming a population of $N$ samples. Sampling from such a population has been discussed for example by Blight (1973). The variance $\sigma_{p}^{2}$ of the population mean $\mu_{\mathrm{n}}=\frac{1}{\mathrm{n}} \sum_{i=1}^{\mathrm{n}} \varepsilon_{i}$ is

$$
\sigma_{p}^{2}=\mathbf{E}\left[\mu_{\mathrm{n}}^{2}\right]-\mathbf{E}\left[\mu_{\mathrm{n}}\right]^{2}=\sigma^{2}\left(\frac{1}{\mathrm{n}} \frac{1+\rho}{1-\rho}-\frac{2 \rho}{\mathrm{n}^{2}} \frac{1-\rho^{\mathrm{n}}}{(1-\rho)^{2}}\right) .
$$

In case the population mean is estimated by a single sample $\varepsilon_{i}$, then the error variance of the estimate mean is

$$
\sigma_{i}^{2}=\mathbf{E}\left[\left(\varepsilon_{i}-\mu_{\mathrm{n}}\right)^{2}\right]=\sigma^{2}-\frac{2}{\mathrm{n}} \frac{\rho\left(1-\rho^{i-1}\right)+\left(1-\rho^{\mathrm{n}-i+1}\right)}{1-\rho}+\sigma_{p}^{2} .
$$

This has a minimum at $i=\mathrm{n} / 2$. This shows that even for a uniform velocity profile, where there is no bank boundary layer, a measurement near the channel centre yields a more accurate discharge estimate than a measurement near the bank (Figure 2.14a).

Averaged over the population, the error variance is

$$
\overline{\sigma_{i}^{2}}=\mathbf{E}\left[\varepsilon_{i}^{2}\right]-\mathbf{E}\left[\mu_{\mathrm{n}}^{2}\right]=\sigma^{2}-\sigma_{p}^{2} .
$$

Hence the finite population size and correlation yields an error variance that is lower than the process variance.

If the population mean is estimated as the mean $\mu_{\mathrm{m}}=\frac{1}{\mathrm{~m}} \sum_{i=1}^{\mathrm{m}} \varepsilon_{i}$ of a subset of the population sampled over a range starting at either end, then the error variance $\sigma_{\mathrm{m}}^{2}$ of the sample mean with respect to the population mean is

$$
\begin{aligned}
\sigma_{\mathrm{m}}^{2}= & \mathbf{E}\left[\left(\mu_{\mathrm{m}}-\mu_{\mathrm{n}}\right)^{2}\right] \\
= & \sigma^{2}\left(\frac{\mathrm{n}-\mathrm{m}}{\mathrm{nm}} \frac{1+\rho}{1-\rho}-2 \frac{\rho}{(1-\rho)^{2}}\left(\frac{1}{\mathrm{~m}^{2}}\left(1-\rho^{\mathrm{m}}\right)\right.\right. \\
& \left.\left.\quad-\frac{1}{\mathrm{~nm}}\left(1-\rho^{\mathrm{m}}+\rho^{\mathrm{n}-\mathrm{m}}-\rho^{\mathrm{n}}\right)+\frac{1}{\mathrm{n}^{2}}\left(1-\rho^{\mathrm{n}}\right)\right)\right) .
\end{aligned}
$$

This formula is also valid in the special case of no correlation $(\rho=0)$ or a cross section with unlimited width $(n \rightarrow \infty)$. As the profiling range increases, the 
sample size approaches the population size $(m \rightarrow n)$, and the error goes to zero (Figure 2.14b). This gives the factors $g_{t}$ and $g_{v}$ in eq. (2.11) as the ratio of the error variance of the correlated finite process as well as of the uncorrelated innumerable process $g=\frac{\sigma_{\mathrm{m}}^{2}}{\sigma^{2}}$.

The auto-covariance function at lag $k$ is

$$
\begin{aligned}
a_{k}= & \mathbf{E}\left[\left(\varepsilon_{i}-\mu_{\mathrm{n}}\right)\left(\varepsilon_{i+k}-\mu_{\mathrm{n}}\right)\right], \\
= & \sigma_{\mathrm{n}}^{2}+\sigma^{2} \rho^{k} \\
& -2 \sigma^{2}\left(\frac{1}{\mathrm{n}}\left(\frac{1+\rho}{1-\rho}+\frac{1}{N-k} \frac{\rho}{(1-\rho)^{2}}\left(1-\rho^{\mathrm{n}-k}+\rho^{k}-\rho^{\mathrm{n}}\right)\right)\right) .
\end{aligned}
$$

To obtain an unbiased estimate, the covariances are normalized with $\frac{1}{N-k}$. To calculate these factors, the population size $\mathrm{n}$ has to be defined. The finite width of the cross section only affects the transverse profile of the velocity, which has a population size of $\frac{w}{\Delta n}$. The error estimate is independent of $\Delta n$. As no averaging over the vertical takes place, the population size of for the vertical profile is the limit $\mathrm{n} \rightarrow \infty$.

\subsubsection{Cross-correlation of two spatially correlated populations}

Consider now the bivariate case, to model the combined error due to variation of the transverse and vertical profile scale. Consider two processes generated by equation (2.26a) with variances $\sigma_{1}$ and $\sigma_{2}$ as well as correlations $\rho_{1}$ and $\rho_{2}$. Let the innovations $\varepsilon_{1, i}$ and $\varepsilon_{2, i}$ be mutually correlated by the correlation matrix $R$ :

$$
R=\left[\begin{array}{cc}
1 & 1-\tilde{\rho}_{12} \\
0 & \sqrt{1-\tilde{\rho}_{12}^{2}}
\end{array}\right]
$$

where the cross-correlation is scaled as $\tilde{\rho}_{12}=\rho_{12} \frac{1-\rho_{1} \rho_{2}}{\sqrt{1-\rho_{1}^{2}} \sqrt{1-\rho_{2}^{2}}}$. Then at lag $k$ the covariance between the two series is

$$
\rho_{12, k}=\mathbf{E}\left[\varepsilon_{1, i} \varepsilon_{2, i+k}\right]=\rho_{12} \sigma_{1} \sigma_{2}\left\{\begin{array}{cc}
\rho_{1}^{-k}, & k \leq 0 \\
\rho_{2}^{k}, & k \geq 0
\end{array} .\right.
$$


The factor $g_{t, v}$ of the covariance term in equation (2.11) is $\frac{\mathbf{E}\left[\left(\varepsilon_{1, i}-\mu_{1}\right)\left(\varepsilon_{2, j}-\mu_{2}\right)\right]}{\rho_{12} \sigma_{1} \sigma_{2}}$, with

$$
\begin{gathered}
\mathbf{E}\left[\left(\varepsilon_{1, i}-\mu_{1}\right)\left(\varepsilon_{2, j}-\mu_{2}\right)\right]=\rho_{12} \sigma_{1} \sigma_{2}\left(\frac{\mathrm{n}_{1}-\mathrm{m}}{\mathrm{n}_{1} \mathrm{~m}} \frac{1-\rho_{1} \rho_{2}}{\left(1-\rho_{1}\right)\left(1-\rho_{2}\right)}\right. \\
-\frac{\rho_{1}}{\left(1-\rho_{1}\right)^{2}}\left(\frac{1-\rho_{1}^{\mathrm{m}}}{\mathrm{m}^{2}}+\frac{1-\rho_{1}^{\mathrm{n}_{1}}}{\mathrm{n}_{1} \mathrm{n}_{2}}-\frac{1-\rho_{1}^{\mathrm{m}}}{\mathrm{m} \mathrm{n}_{2}}-\rho_{1}^{\mathrm{n}_{1}} \frac{\rho_{1}^{-\mathrm{m}}-1}{\mathrm{~m} \mathrm{n}_{1}}\right) \\
\left.-\frac{\rho_{2}}{\left(1-\rho_{2}\right)^{2}}\left(\frac{1-\rho_{2}^{\mathrm{m}}}{\mathrm{m}^{2}}+\rho_{2}^{\mathrm{n}_{2}} \frac{\rho_{2}^{-\mathrm{n}_{1}}-1}{\mathrm{n}_{1} \mathrm{n}_{2}}-\frac{1-\rho_{2}^{\mathrm{m}}}{\mathrm{m} \mathrm{n}_{1}}-\rho_{2}^{\mathrm{n}_{2}} \frac{\rho_{2}^{-\mathrm{m}}-1}{\mathrm{~m} \mathrm{n}_{2}}\right)\right) .
\end{gathered}
$$


2 


\section{Distributary channels in the fluvial to tidal transition zone}

Castal lowland plains under mixed fluvial-tidal influence may form comacteristics of mouth bar channels, avulsion channels and tidal creeks. The Kapuas coastal plain exemplifies such a coastal plain, where several narrow distributaries branch off the Kapuas River at highly asymmetric bifurcations. A comprehensive geomorphological analysis shows that trends in the channel geometry of all Kapuas distributaries are similar. They consist of a short, converging reach near the sea and a non-converging reach upstream. The two parts are separated by a clear break in scaling of geometrical properties. Such a break in scaling was previously established in the Mahakam Delta, which suggests that this may be a general characteristic in the fluvial to tidal transition zone. In contrast to the geometrical trend similarities, a clear difference in bed material between the main and side distributaries is found. In the main distributary, a continuous trend of downstream fining is established, similar to what is often found in lowland rivers. In the side distributaries, bed material coarsens in the downstream direction. This indicates an undersupply of sediment to the side distributaries, which may contribute to their long-term stability as established from historical maps. Tides may be the main agent preventing fine sediment to settle, promoting residual transport of fine material to the coastal ocean.

This chapter is based on: Kästner, K., A. J. F. Hoitink, B. Vermeulen, T. J. Geertsema, and N. S. Ningsih, Distributary channels in the fluvial to tidal transition zone, Journal of Geophysical Research: Earth Surface, 3(122), 696-710, 2016JF004075, 2017 


\subsection{Introduction}

River deltas are formed by deposition and reworking of sediment (Davis Jr and Dalrymple, 2011). Deltas take a characteristic appearance depending on the degree in which river discharge, wind waves, and tides contribute to sediment transport and morphological change (Galloway, 1975). Across a single delta plain, the influence of river flow, wave action, and tides can vary, which locally affects the appearance of the delta. For example, the Mahakam forms a typical mixed river-tide dominated delta, with tidal channels often disconnected from fluvial distributaries (Figure 3.1). The tidal channels are highly sinuous and converge strongly, i.e., their cross section area decreases exponentially in upstream direction, which is in contrast to most of the distributaries. Sassi et al. (2012) revealed a break in cross section geometrical properties in the Mahakam distributaries, which separates tide-dominated reaches downstream from the river-dominated reaches further upstream. Here, we report on a channel network which shows a similar break in scaling behaviour, and investigate the link with trends in grain size development. Our aim is to better understand downstream trends in channel geometrical properties and bed material grain size across the fluvial to tidal transition zone by focusing on the Kapuas lowland plain. The Kapuas forms a complex delta channel network (Figure 3.2). Individual distributaries blend the properties of mouth bar channels, avulsion channels and tidal creeks. These three channel types are defined in greater detail hereafter.

Mouth bar channels are formed during delta progradation (Olariu and Bhattacharya, 2006) as a consequence of continuous deposition of sediment in front of distributary outlets. These mouth bar deposits develop into islands over time, after which two new channels bifurcate around them (Mikhailov, 1966; Edmonds and Slingerland, 2007). If undisturbed by waves and tides, distributary channels recursively bifurcate around mouth bars during progradation, forming a delta with channels radiating from a single delta apex (Andren, 1994; Mikhailov, 1970). The resulting distributary channel network features a fractal appearance (Marciano et al., 2005). Due to deposition at the mouth, the distributaries are wide and shallow, and due to decreasing discharge with increasing bifurcation order, width and depth decrease in the downstream direction. Mouth bar formation requires sufficient inflow and sediment supply from the river (Edmonds and Slingerland, 2007). The Wax Lake delta is a typical example of a delta composed of mouth bar channels (Roberts et al., 1980). Interaction between the radial spreading of the flows from neighbouring distributary channels can form bathymetric depressions termed interdistributary 

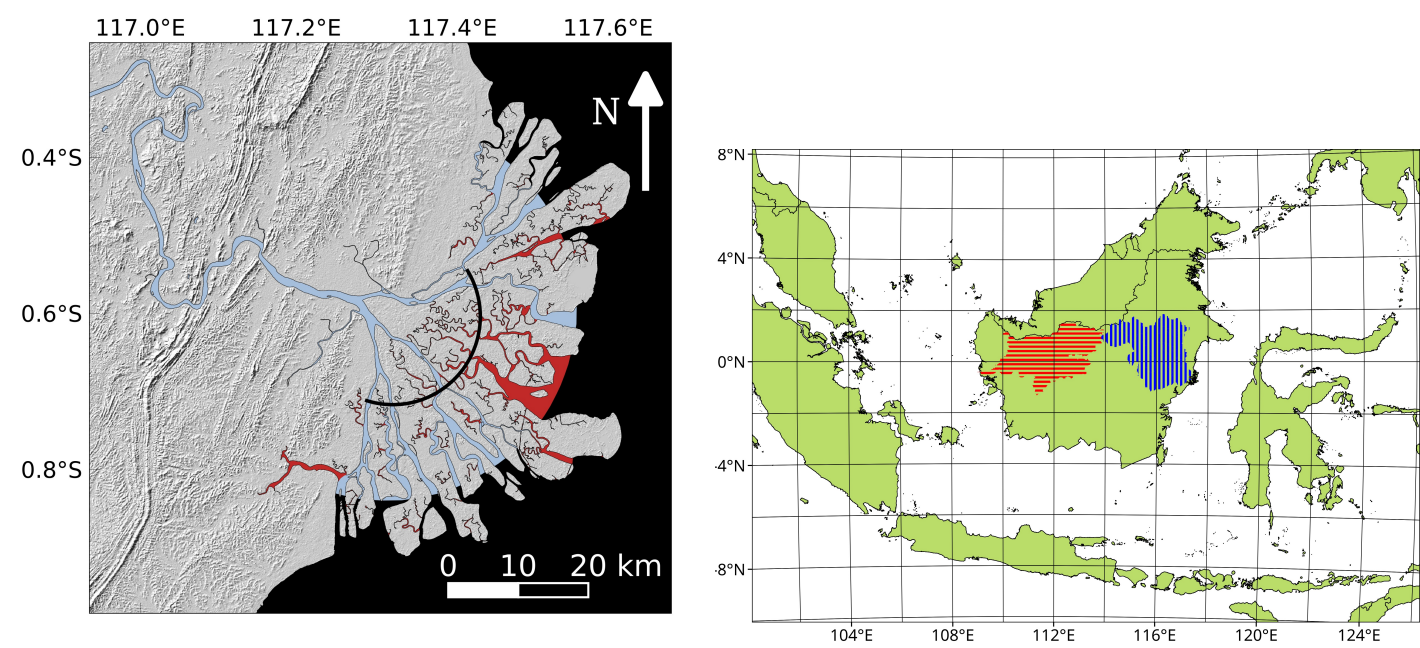

Figure 3.1: Left: Distributaries (blue) and tidal channels (red) in the Mahakam Delta. Gray shading indicates relief from SRTM data. The semicircle corresponds to the distance from the delta apex where the channel geometry changes from from fluvial to tidal form (adapted from Sassi et al. (2012)). Right: Location of the Kapuas (red horizontally hatched) and Mahakam (blue vertically hatched) catchments on the island of Borneo

troughs, which can complicate the channel pattern (Shaw et al., 2016).

Distributary channels can also be created by avulsion, when, mostly during large flood events, the river bifurcates and takes a new course along a path with slope advantage (Slingerland and Smith, 1998; Stouthamer et al., 2011). Once a new avulsion channel is established, the old distributary may slowly be abandoned. This process can lead to cyclic delta complex switching, which occurs for example in the Mississippi (Coleman, 1988). If avulsions occur more frequently than channels are abandoned, an anastamosing pattern with multiple channels running along the alluvial plain develops, extending the delta far upstream of the mouth bar complex (Makaske, 2001). Albeit part of the delta architecture where waves and tides prevail, these distributaries often remain of fluvial nature. There is net sediment throughput in stable conditions, and their width-to-depth ratio is similar to that of single thread rivers. Anastamosing channel patterns can be found in many deltas around the world, for example in the Parana (Stevaux and Souza, 2004), the Mackenzie (Emmerton et al., 2007), the Pearl (Wang et al., 2004), and the Lena (Costard and Gautier, 2007).

Tidal creeks can grow by headward erosion on tidal flats. They can be sole conveyors of tides and local runoff and do not need to receive river inflow (D’Alpaos et al., 2005). Tidal channels often form dentritic networks, where 


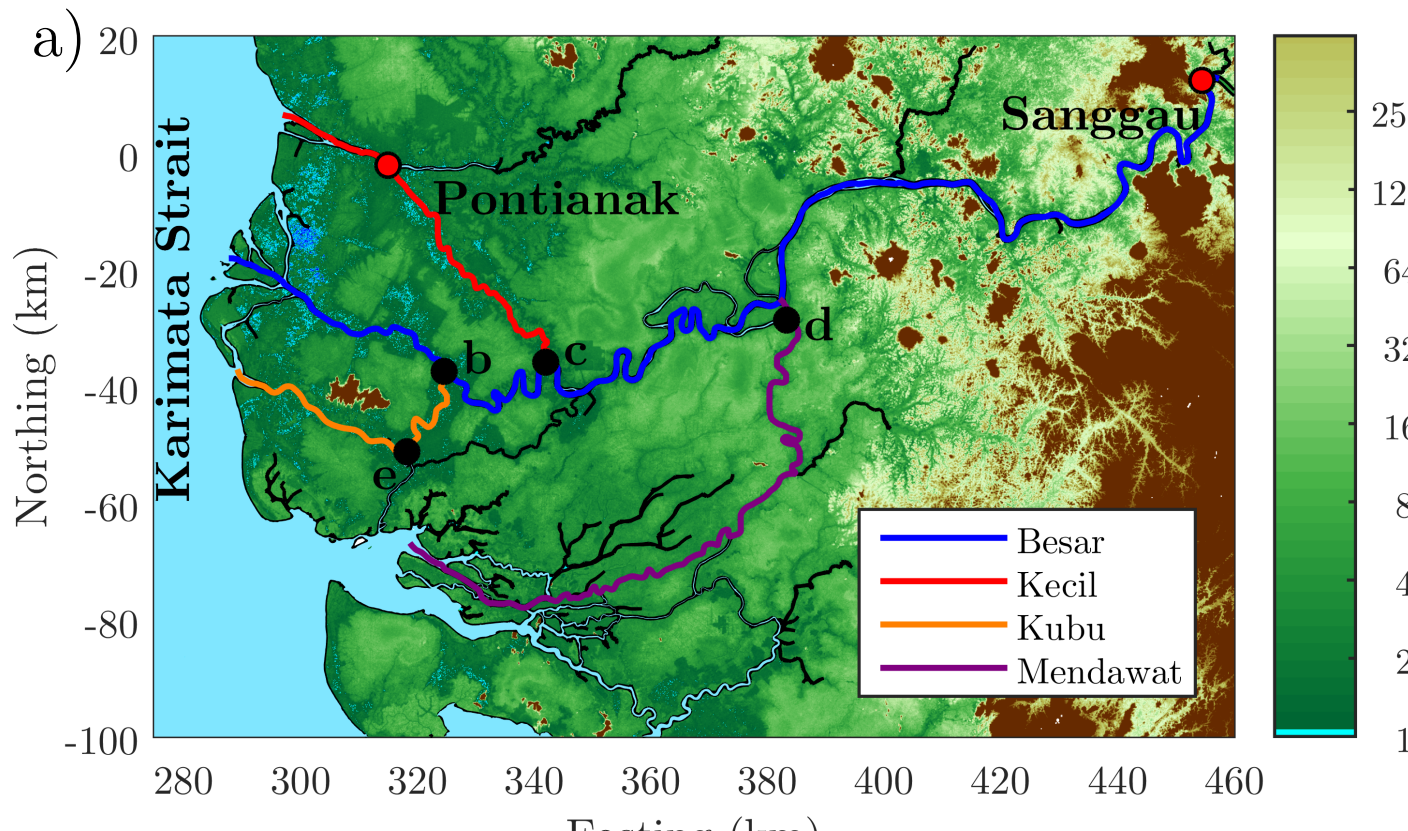

Easting $(\mathrm{km})$

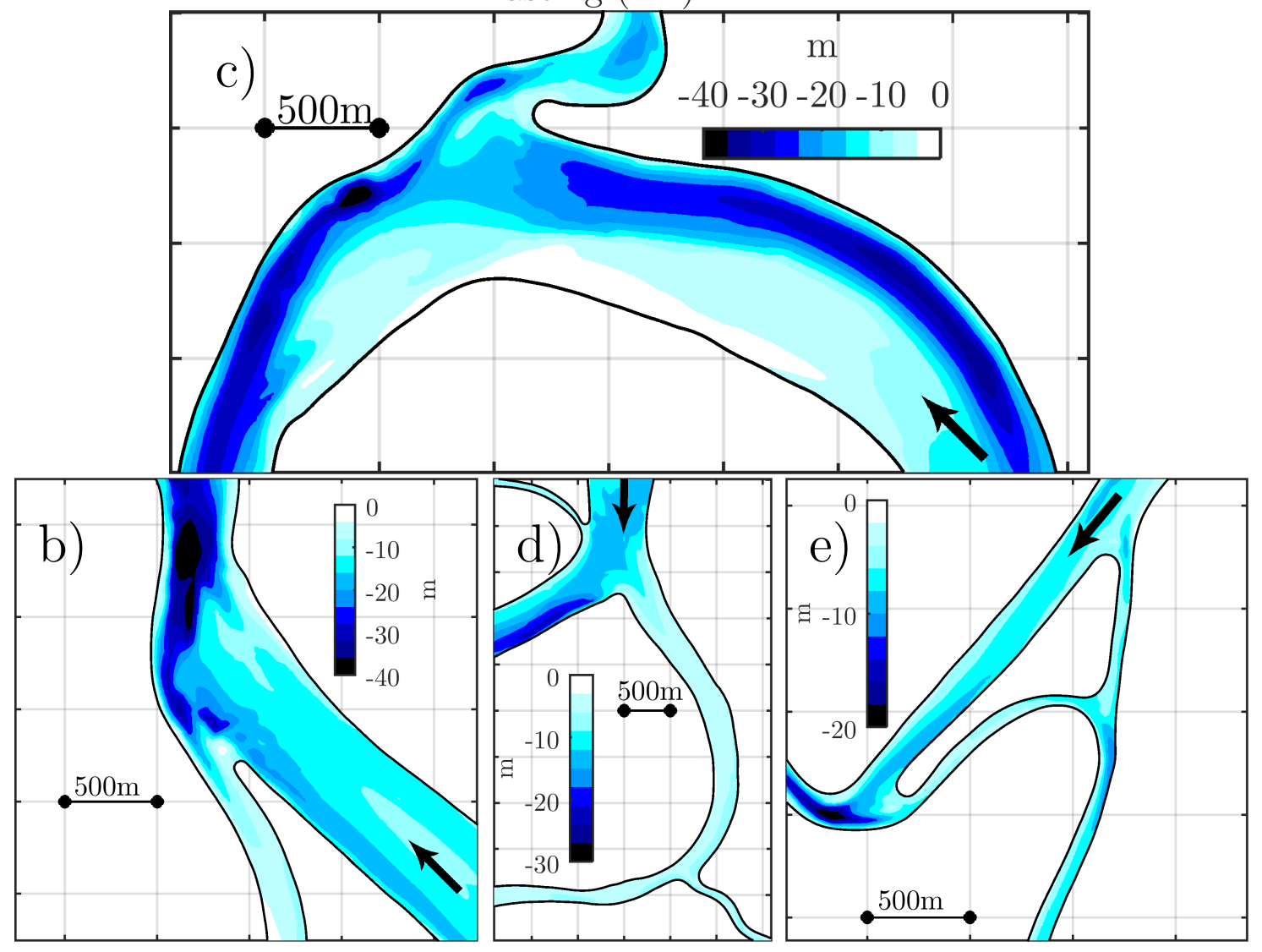

Figure 3.2: Topography of the Kapuas coastal plain with elevation from SRTM data (a); bathymetry of bifurcations: Kapuas Besar - Kubu (b), Kapuas Besar - Kapuas Kecil (c), Kapuas Besar - Kubu (d), and South Kubu (e, neck cut-off due to dredging)

62 Chapter 3. Distributary channels in the fluvial to tidal transition 
channels recursively branch off in the upstream direction at bifurcations with right angles (Dalrymple et al., 2012). These channels often interconnect to form islands (Fagherazzi, 2008). The inlet size of a tidal channel is related to the tidal prism (Jarrett, 1976; D'Alpaos et al., 2009), and width strongly converges with the reduction of the tidal prism in the upstream direction (Pillsbury, 1940; Myrick and Leopold, 1963; Wright et al., 1973; Fagherazzi and Furbish, 2001; Davies and Woodroffe, 2010). Typically, tidal channels are strongly meandering; their curvature decreases towards the channel outlet. The shape of the meander loops is more symmetric than that of fluvial meanders, due to the bidirectional flow (Marani et al., 2002). If there is no inflow from upstream, then there is no net sediment transport in stable tidal channels, similar to equilibrium estuaries (Seminara et al., 2012). The width-to-depth ratio of tidal channels is lower than that of mouth bar distributaries (Marani et al., 2002). Contrary to mouth bar distributaries, the depth of tidal channels increases in downstream direction with increasing gross discharge, although the depth convergence is smaller than the width convergence (Savenije et al., 2008). The Sundarbans represent one of the most extensive tidal creek systems worldwide, located in the Ganges-Brahmaputra Delta (Passalacqua et al., 2013).

The along-channel trends of discharge differ between mouth bar channels, tidal creeks and avulsion channels. As the discharge changes, the cross section geometry adapts accordingly. The concept of downstream hydraulic geometry can be used for channel comparison. In hydraulic geometry, the relations between hydraulic parameters such as width and depth on discharge are expressed as power laws. In general, width and depth increase with increasing discharge (Leopold and Maddock Jr, 1953). A recent review was compiled by Eaton (2013). Similar to the channel geometry, the bed material grain size often has a distinct along-channel trend, which can be used to identify the morphological setting. Grain size of river sediment typically decreases in the downstream direction, due to abrasion and selective transport (Snow and Slingerland, 1987; Hack, 1957; Frings, 2008). As the transport of sediment depends on the grain size, it influences the planform of fluvial channels (Schumm, 1985) and affects the morphology of deltas (Orton and Reading, 1993; Geleynse et al., 2011). Empirical hydraulic relationships including grain size have been proposed for fluvial channels (Wilkerson and Parker, 2010), yet little is known about the grain size trends in the fluvial to tidal transition zone, where bidirectional flow may cause a local maximum of grain size in estuaries (Dalrymple and Choi, 2007). 


\subsubsection{Field site}

The Kapuas is the largest river on the island of Borneo with a catchment size of $9.9 \cdot 10^{4} \mathrm{~km}^{2}$. It is draining into the shallow Karimata strait, which separates Borneo from mainland Asia and the island Sumatra. The discharge is subject to strong seasonal variation associated with the monsoon (Hidayat et al., 2017). The Kapuas River is the main waterway in West Kalimantan, serving inland transportation. With the harbour of Pontianak at its mouth, it connects the province to the archipelago. The city of Pontianak relies on the river as a source of fresh water and fish, but is subject to salinity intrusion and flooding during seasonal extremes. The Kapuas river is not yet controlled by manmade structures. Human influence is mostly indirect such as by development of palm oil plantations, which quickens rainfall runoff and increases sediment supply (Buschman et al., 2012). Despite the widespread development of oil palm plantations, the Kapuas River system can still be considered a relatively pristine setting.

Between 2013 and 2015, the Kapuas River was surveyed from the sea to the city of Sanggau, located $300 \mathrm{~km}$ upstream. Throughout this paper, distances are given along the river with respect to Sanggau. Where appropriate, the corresponding distances to the sea are provided in parentheses, e.g., Sanggau $\mathrm{km} 0$ ( $\mathrm{km} \mathrm{300).} \mathrm{For} \mathrm{the} \mathrm{first} 100 \mathrm{~km}$ downstream of Sanggau, the Kapuas flows through a valley before entering the alluvial plain. Along the alluvial plain, several small distributaries branch off and the river forms a complex channel network (Figure 3.2), although most discharge reaches the sea through the main distributary: the Kapuas Besar. The bifurcations are highly asymmetric, such that the flow entering the side channel curves around a spit (Figure 3.2a$3.2 \mathrm{~d}$ ). The main distributary terminates in a delta fan with $15 \mathrm{~km}$ radius. The delta fan has one large trifurcation just downstream of the apex and five outlets. The three small distributaries are named Mendawat, Kapuas Kecil and Kubu. They branch off at km 130 (159), 203 (87) and 241 (49), as indicated in Figure 3.2. The Kapuas Besar is meandering and has no major confluences downstream of Sanggau. The Mendawat and Kubu flow towards the South and are connected to a network of tidal creeks. The small distributaries show a meander pattern similar to the Kapuas Besar, and receive a disproportionately large inflow, with respect to their size.

During the monitoring period from October 2013 to May 2015 the daily averaged discharge ranged between $900 \mathrm{~m}^{3} \mathrm{~s}^{-1}$ and $9500 \mathrm{~m}^{3} \mathrm{~s}^{-1}$ at Sanggau. The water level range exceeds $9 \mathrm{~m}$ at Sanggau and gradually decreases in downstream direction to $2 \mathrm{~m}$ at $\mathrm{km} \mathrm{150.} \mathrm{At} \mathrm{this} \mathrm{point,} \mathrm{the} \mathrm{downstream} \mathrm{curve} \mathrm{of}$ 
the water level range has a pronounced break (Figure 3.3a). Averaged over a Metonic cycle, the tidal range in the Karimata Strait at the Kapuas River mouth is $1.35 \mathrm{~m}$ at spring tide and $0.35 \mathrm{~m}$ at neap tide (Figure 3.3b). The tidal form factor is 2.5 , based on which the tides can be classified as mixed, mainly diurnal (Pugh, 1987). Propagation of tides in the river strongly depends on discharge (cf. Hoitink and Jay, 2016). Similarly to the water level range, the tidal amplitude development has a pronounced drop at the transition between the alluvial plain and the upstream valley. At low flow, the tide propagates until km 130 (160) without being considerably attenuated. From there on a gradual attenuation sets in (Figure 3.3c), so that the tidal amplitude in Sanggau is still $40 \%$ of the tidal amplitude at sea. At normal flow, the tide is also attenuated along the alluvial plain, but the attenuation is stronger beyond $\mathrm{km} 130$ so that it does not reach Sanggau. At high flow, tides are gradually damped from the sea and fade out at $\mathrm{km} 130$.
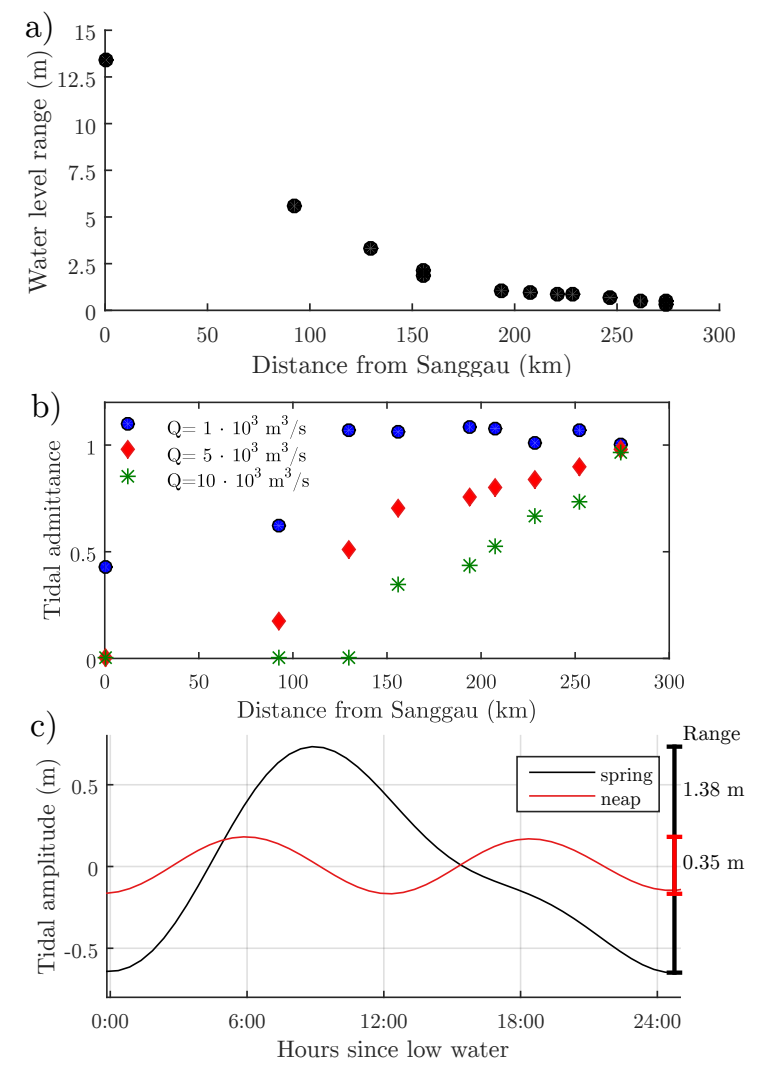

Figure 3.3: Water level range (a), admittance of the tidal wave along the Kapuas River (b), predicted spring (black) and neap (red) tide at the Kapuas River mouth (Egbert and Erofeeva, 2002) (c) 


\subsection{Methods}

\subsubsection{Field data collection}

Our survey of the Kapuas River reached from the sea until Sanggau, including the Kapuas Besar, Mendawat, Kapuas Kecil and Kubu distributaries. The survey lasted from October 2013 to April 2015. Flow conditions ranged from base flow to bankfull flow during this period. The bathymetry was surveyed with a single beam echo sounder. We measured the bathymetry one time by crossing the river at an interval of one river width and a second time by following the river along its centre (Vermeulen et al., 2014a). Only a basic GPS with low vertical accuracy was available during the initial survey. An additional survey with a carrier phase and TerraStar-corrected GPS took place in November 2016. This survey consisted only of two along river tracks. The two tracks followed the left and right river bank, respectively, with an approximate distance to the river bank of $1 / 4^{\mathrm{er}}$ of the cross section width. We determined the bankfull depth by combining the sounded bathymetry with surface level measurements recorded at 15 stations across the study area. Bed material was sampled with a Van Veen grabber. Three samples across the river were collected every $2.5 \mathrm{~km}$, in total 411 samples. In the fan-shaped delta, bed material was sampled only in the central channel. At a few locations of interest, the sampling density was increased and at several other locations no samples could be retrieved, presumably due to the presence of compact clay, gravel or wooden debris. Samples were dried and sieved into separate size classes. For each sample, $200 \mathrm{~g}$ dry material was processed. Cohesive samples were crushed before sieving. ASTM E11 sieves were used with a mesh size ranging between 0.045 and $4.75 \mathrm{~mm}$, spaced by a factor of 1.4 (American Society for Testing and Materials E11, 2009).

\subsubsection{Data analysis}

Channel width and curvature were extracted from Landsat images (Courtesy of the U.S. Geological Survey, data available at https : //earthexplorer. usgs . gov). At first, pixels were classified either as land or water, and then the bank line was extracted at the interface. Sounded depth was transformed to bankfull water level with the instantaneous water level (Figure 3.4). We employ the model by Kukulka and Jay (2003b,a) to infer the instantaneous water level from the stage measured at Sanggau and tide predicted in the Karimata strait, and use the data collected at the gauging stations for model calibration. Bankfull water level was defined as the water level averaged over a lunar day occurring 
during high flow $\left(10^{4} \mathrm{~m}^{3} \mathrm{~s}^{-1}\right)$. Error in the conventional GPS altitude with respect to the bed elevation change along the surveyed reach inhibited accurate vertical referencing of the bed elevation. The echo sounder data were resampled to a $10 \mathrm{~m}$ resolution and then interpolated to a mesh. The cross section area was determined at a $50 \mathrm{~m}$ interval by integrating the depth across the section. Channel depth is here defined as the ratio of area and top width. Due to the absence of extensive mudflats and flood plains, the channel widths are fairly constant in the studied area.

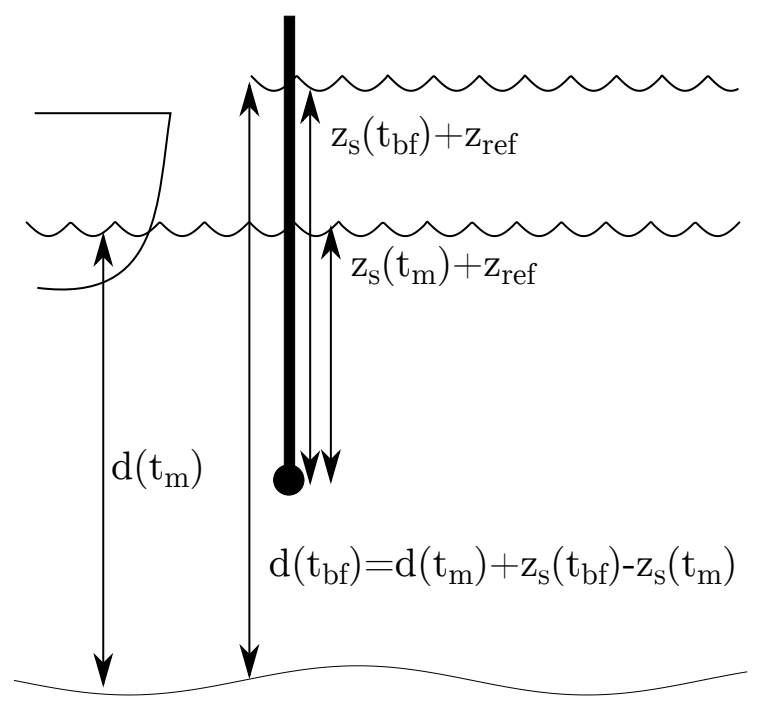

Figure 3.4: Determination of the bankfull depth from the depth sounded at an arbitrary water level; The depth at times of bankfull flow $d\left(t_{\mathrm{bf}}\right)$ is increased by the difference of the water level at times of bank full flow $z_{s}\left(t_{\mathrm{bf}}\right)$ and the water level at the time of measurement $z_{s}\left(t_{m}\right)$. The altitude $z_{\text {ref }}$ of the water level gauge with respect to the geodetic datum does not need to be known.

We analyzed the field data in two ways: first, by spatially filtering the parameters of channel geometry and grain size. Regarding the channel geometry, width $(W)$, bankfull depth $(D)$, aspect ratio $(W / D)$, cross section area $(W \cdot D)$ and curvature $(C)$ were analyzed. Those parameters of channel geometry were sampled at a $50 \mathrm{~m}$ interval and then spatially filtered with a weighted quantile filter. A Hanning-window of $20 \mathrm{~km}$ length was used as a filter window. Within the filter range, the weighed median as well as the $16^{\text {th }}$ and $84^{\text {th }}$ quantile were determined. A quantile filter was used as it is robust to outliers and preserves breaks (Arce et al., 1998). We explain the filter algorithm in detail in the supporting information. Regarding channel curvature, the filter was applied to the absolute value. Parameters of hydraulic geometry were plotted on logarithmic scale to the basis two. The bed level with respect to the WGS 84 was 
determined from the additional bathymetry data collected in 2016. The alongriver tracks of this measurement were filtered with a weighed median filter and a Hanning window of $20 \mathrm{~km}$ length to remove perturbations by bed forms and scour holes.

For each sample, the median $\left(D_{50}\right), 16^{\text {th }}\left(D_{16}\right)$, and $84^{\text {th }}\left(D_{84}\right)$ percentiles as well as the standard deviation and skewness of the grain size distribution were computed on log-scale. Standard deviation and skewness are given as non-dimensional grain size $\log _{2}(D /(1 \mathrm{~mm}))$ and for clarity denoted as $\phi$. Grain size itself is for better readability retransformed to millimetres. As for the channel geometry parameters, the grain size parameters were spatially filtered with a weighed quantile filter. In addition, the histograms of the grain size distribution were averaged for each reach and plotted side by side. For each histogram bin, the trend along the reach was estimated as the difference of the average histogram in the upstream and downstream half of the reach. This allows to interpret grain size trends in terms of sediment transport and deposition, applying theory of selective transport (McLaren, 1981). As small grains are transported at a higher rate than larger grains, the grain size distribution changes along the direction of transport. McLaren (1981) developed this concept to determine the direction and regime of sediment transport. If the grain size distribution at the source is uniform, the sediment becomes finer, better sorted and more skewed in the downstream direction. As the grain size distribution in the Kapuas are far from uniform, the trends of statistical parameters of the grain size distribution are more complex. There the transport regime is directly derived from the histograms (Figure 3.8), rather than from the statistical moments (Figure 3.6). In addition the parameter distribution for each reach, i.e., the distribution of the mean, standard deviation and skewness of the bed material samples, was then estimated with kernel density functions (Scott, 2015). As a convolution Kernel, a Hanning-window of $1 \phi$ width for the median grain size and $1 / 2 \phi$ for the standard deviation were chosen.

\subsection{Results}

\subsubsection{Channel geometry}

In the main distributary, three reaches with distinct trends of channel geometry can be distinguished. These correspond to the upstream valley, the alluvial plain, and the delta fan. In the upstream valley, channel width increases from $590 \mathrm{~m}$ at Sanggau to $910 \mathrm{~m}$ at the upstream limit of the alluvial plain, which 


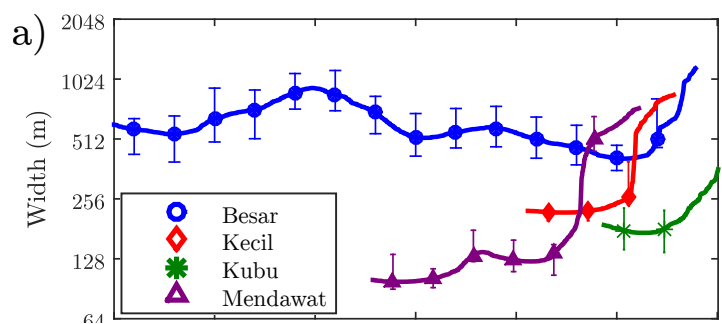

b)
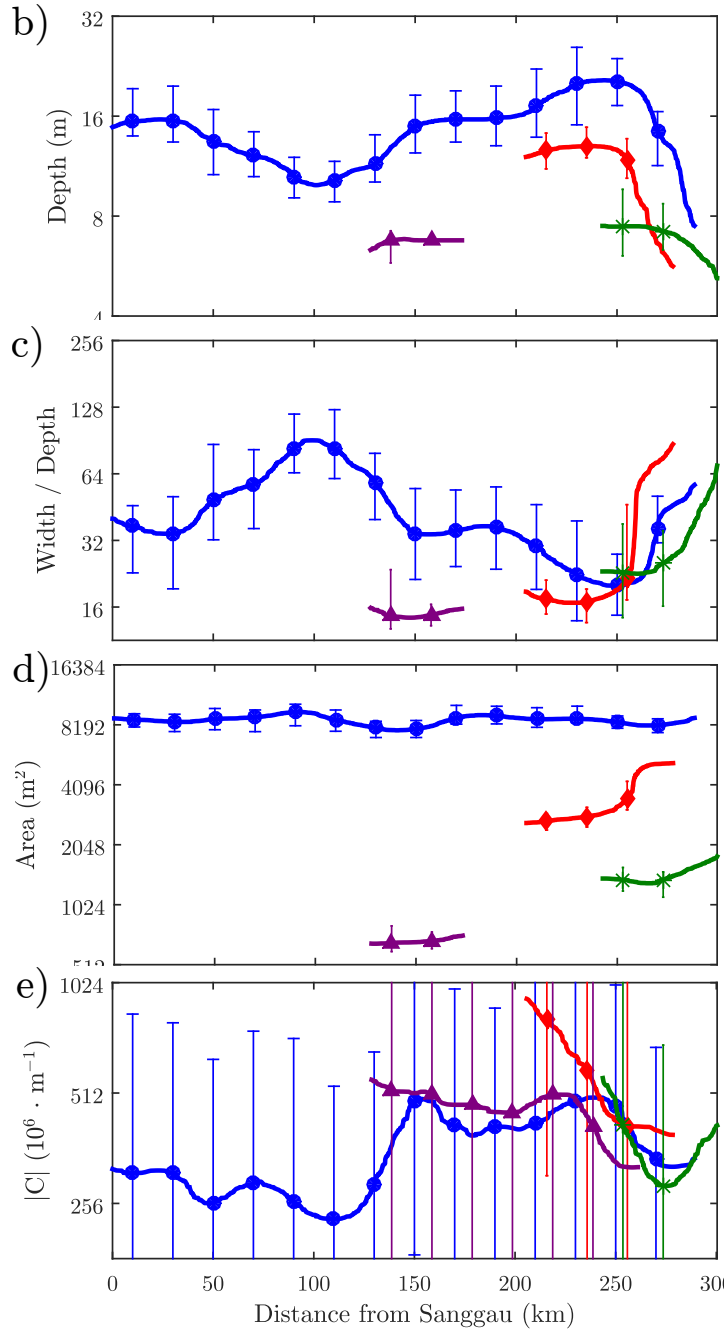

Figure 3.5: Downstream channel geometry of the Kapuas River, the lines indicate the median and candlesticks indicate the $16^{\text {th }}$ and $84^{\text {th }}$ percentile within the filter range; (a) width, (b) depth, (c) width-to-depth ratio, (d) cross section area, and (e) centre-line cuvature.
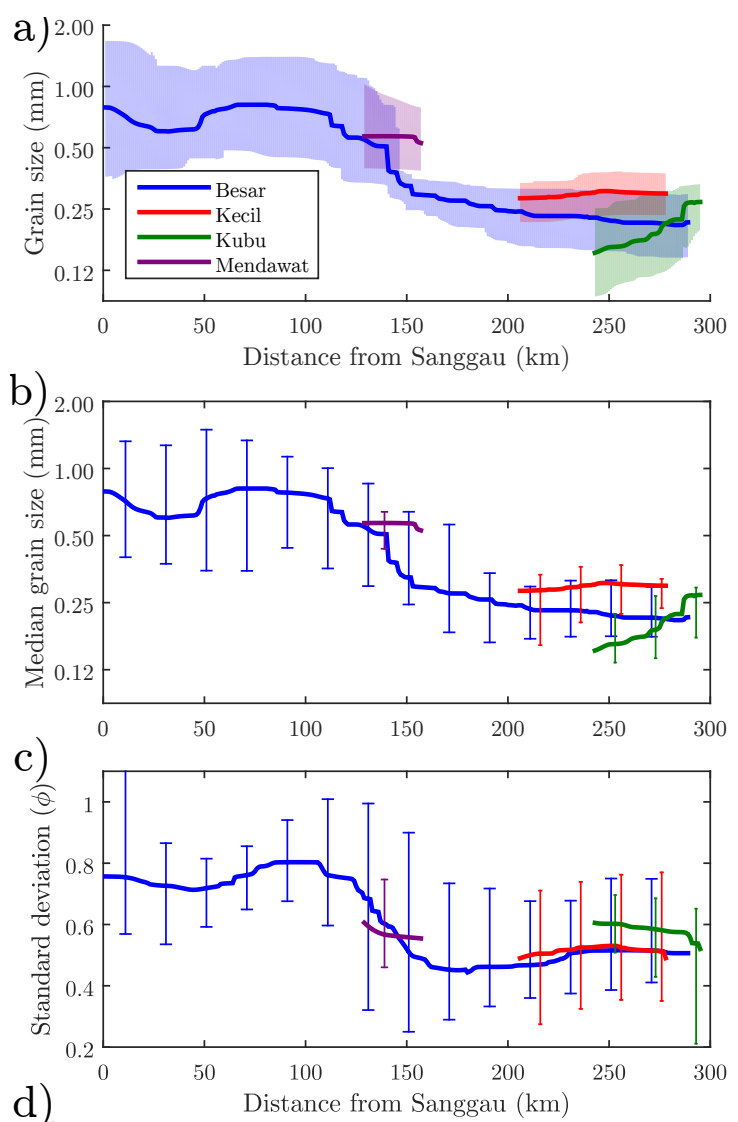

d)

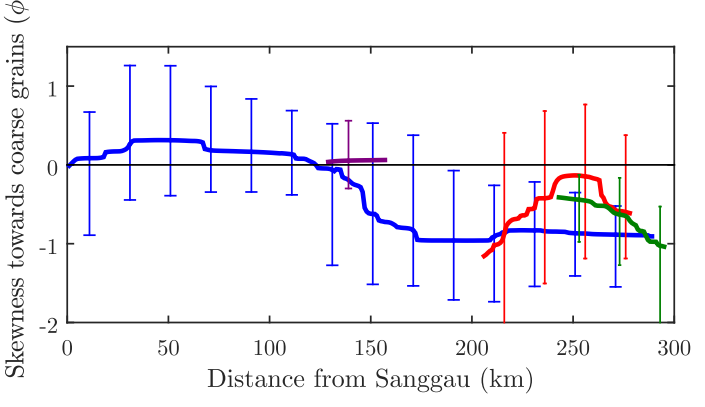

Figure 3.6: Median $D_{50}(\mathrm{a}, \mathrm{b})$, standard deviation (c) and skewness (d) of the bed material grain size distribution along the Kapuas River; Shaded area in a indicates range between $D_{16}$ and $D_{84}$ of the grain size distribution; Candlesticks in b-c indicate the $16^{\text {th }}$ and $84^{\text {th }}$ quantile of the respective parameter within the filter range. 
corresponds to km 100 (190) (Figure 3.5a). Bankfull depth decreases in the latter reach from $15 \mathrm{~m}$ to $10 \mathrm{~m}$ (Figure 3.5b). There is a strong increase of the width-to-depth ratio from 40 to 90 (Figure 3.5c), but only a slight increase of the cross section area from 8793 to $9500 \mathrm{~m}^{2}$ (Figure 3.5d). The transition from the valley to the alluvial plain is also distinguished by an increase of curvature (Figure 3.5e). Along the alluvial plain, the Kapuas gradually narrows and reaches a minimum width of $400 \mathrm{~m}$ at $\mathrm{km} 255$ ( $\mathrm{km} \mathrm{35),} \mathrm{not} \mathrm{far} \mathrm{upstream}$ from the delta apex. Parallel to the decrease in width, the bed level drops (Figure 3.7) so that the depth reaches a maximum of $20 \mathrm{~m}$ at the same place where the width obtains its minimum. Between the bifurcation with the Kapuas Kecil and the delta apex the Kapuas Besar has several deep scour holes with depths up to $55 \mathrm{~m}$. The width-to-depth ratio decreases to 20 along the alluvial plain, due to the opposite trends in width and depth. For the same reason, the cross section area remains nearly constant along the alluvial plain. The curvature first increases in the upstream part of the alluvial plain, and remains high until km 240, from where it reduces gradually towards the sea. From km 255 (km 35) downwards, the Kapuas Besar gradually widens before splitting into the five branches. Summed over the branches, the total width continues to increase along the delta. The depth decreases throughout this last reach. All delta branches terminate in shallow mouth bars, where the bed almost emerges at low tide. As the width increases faster than depth decreases, the cross section area increases towards the sea throughout the delta. However, the area increase is relatively small.

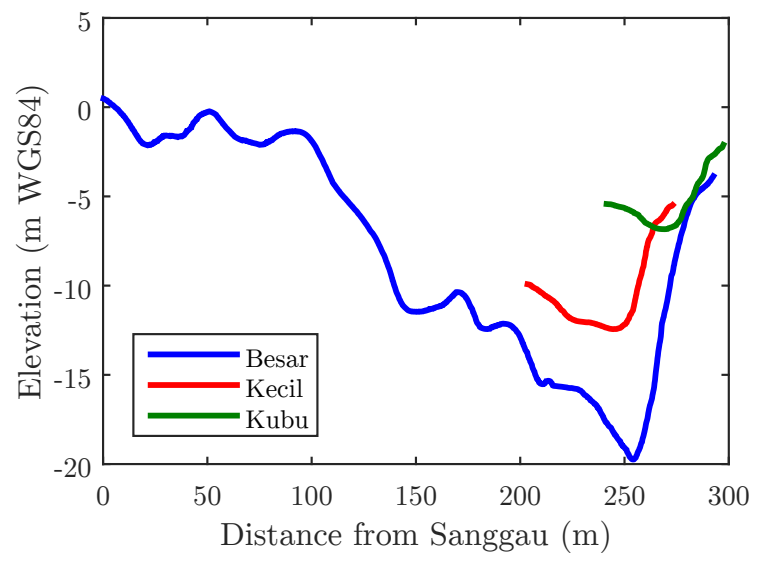

Figure 3.7: Bed level of the Kapuas River, candlesticks indicate $16^{\text {th }}$ and $84^{\text {th }}$ percentile within the filter range of $20 \mathrm{~km}$; detailed bathymetric maps of the bifurcations are provided in Figure 2

The side distributaries are considerably narrower and shallower than the 
Kapuas Besar. The Kapuas Kecil is the largest and the Mendawat is the smallest of the side distributaries. Water level ranges over more than $3 \mathrm{~m}$ at the most upstream bifurcation, where the Mendawat branches off the Kapuas Besar. At this bifurcation, depth and area ratios are more extreme during low flow conditions than those at bankfull flow. The two downstream bifurcations have a particular shape, where the flow curves around a spit before entering the small distributary; see insets in Figure 3.2. The upstream bifurcation is a double bifurcation, where the Mendawat branches off a local avulsion channel, running around an island. Similar to the Kapuas Besar, the upstream parts of the side channels consist each of a fluvial reach with constant cross section and of a funnel-shaped tidal reach that widens towards the sea. The transition is clearly visible as breaks in the downstream trend of width and depth (Figure $3.5 \mathrm{a}$ and b). Upstream of the break, the width-to-depth ratio remains constant along the channel. The transition to the funnel-shaped section in the Kapuas Kecil coincides with the Landak confluence at km 255 ( $\mathrm{km} \mathrm{22),} \mathrm{where}$ the cross section area increases abruptly by $73 \%$. The distance between the bifurcation towards the sea is $12 \mathrm{~km}$ shorter through the Kapuas Kecil than through the Kapuas Besar. The Mendawat is met at a confluence at $\mathrm{km} \mathrm{171,}$ bifurcates at km 191 and further down merges into a tidal creek system. Only the upstream part of the Mendawat was surveyed. Similar to the Kapuas Besar, the surveyed outlets of the Kapuas Kecil and Kubu feature shallow bars before debouching into the Karimata Strait. For reasons of shipping towards the harbour of Pontianak, the mouth of the Kapuas Kecil is regularly deepened by dredging, making it less shallow that of the other distributaries, but this does not remove the overall trend. Depth decreases throughout the funnelshaped reach from $15 \mathrm{~m}$ to $5 \mathrm{~m}$. The curvature development in the Mendawat channel is similar to that of the Kapuas Besar. It remains constant in the upstream reach, before decreasing towards the sea. The curvature values in the Kapuas Kecil and Kubu are higher than in the Kapuas Besar. Both in the Kapuas Kecil and in the Kubu, curvature decreases towards the sea, not far downstream of the bifurcation. There is no break in the downstream trend of curvature.

\subsubsection{Bed material grain size distribution}

Bed material in the Kapuas between Sanggau and the sea consists of coarse to fine sands. Material in sharp outer bends consists of compact clay, with an increasing amount of gravel throughout the upstream valley. At most of these locations, no material could be sampled with the Van Veen grabber. In total, less than $5 \%$ of all samples were void or composed of either gravel or com- 
pact clay. These locations were excluded from the analysis. Although some gravel and bed rock is present in the valley, the bed material consists predominantly of sand and the gravel-sand transition is located further upstream than Sanggau. From Sanggau to $\mathrm{km} 110$ (km 180) the bed material consists of coarse sand, with a median grain size of $0.7 \mathrm{~mm}$ (Figure 3.6a-b). The standard deviation is $0.77 \phi$, and the distribution is skewed towards coarser grains (Figure 3.6c-d). In this reach, there is no considerable downstream trend of the median grain diameter, standard deviation or skewness (Figure 3.6). At the foot of the alluvial valley, rapid fining sets in. Between km 110 and 150 grains larger than $0.35 \mathrm{~mm}$ are removed from the grain size distribution. In this reach, along-channel changes of the grain size distribution are much larger than in the reaches further up- and downstream (Figure 3.8). The removal of coarse material causes an even more rapid decrease of the standard deviation and makes the grain size distribution skewed towards finer grains. Downstream of $\mathrm{km} \mathrm{150,} \mathrm{the} \mathrm{material} \mathrm{consists} \mathrm{of} \mathrm{medium} \mathrm{and} \mathrm{fine} \mathrm{sand.} \mathrm{The}$ median grain size decreases from $0.30 \mathrm{~mm}$ to $0.22 \mathrm{~mm}$ at the river mouth. The largest size fraction remains close to $0.35 \mathrm{~mm}$, the $D_{84}$ decreases only marginally from $0.35 \mathrm{~mm}$ to $0.30 \mathrm{~mm}$, while the $D_{16}$ decreases from $0.26 \mathrm{~mm}$ to $0.14 \mathrm{~mm}$. Downstream fining continues throughout the delta fan. Upstream of the mouth bar, the grain size increases slightly. The standard deviation between $\mathrm{km} 150$ and the outlet is $0.48 \phi$ and slightly increases towards the sea. The grain size distributions are skewed towards finer grains. Downstream of

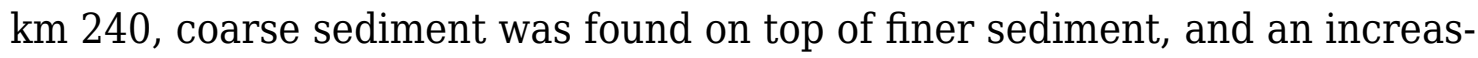
ing number of samples concreted during drying, although only a few samples contained fractions smaller than $65 \mu \mathrm{m}$. The amount of silt could be slightly underestimated, as it is known that dry sieving is not suitable for analysis of silt samples (Xiaoqing and Yang, 2003). The standard deviation of the grain size distribution does not continuously decrease in the downstream direction. This can also be seen in the bivariate plot of median grain size and standard deviation (Figure 3.9b). Samples of intermediate size are better sorted than samples consisting of fines and large material. The standard deviation decreases with the median grain size, up to $0.35 \mathrm{~mm}$, but increases again for decreasing median grain sizes below $0.35 \mathrm{~mm}$. The local variation of the grain size within the range of the filter window is large compared to the change due to downstream fining. Coarse and fine samples were often found within the same cross section. Between $\mathrm{km} 150$ and the mouth, the $16^{\text {th }}$ and $84^{\text {th }}$ percentile of the median grain size of individual samples are $0.35 \mathrm{~mm}$ and $0.18 \mathrm{~mm}$ (Figs. 3.6b and 3.9e). Along-channel trends explain only half of the variance between samples $\left(R^{2}=0.52\right)$. 

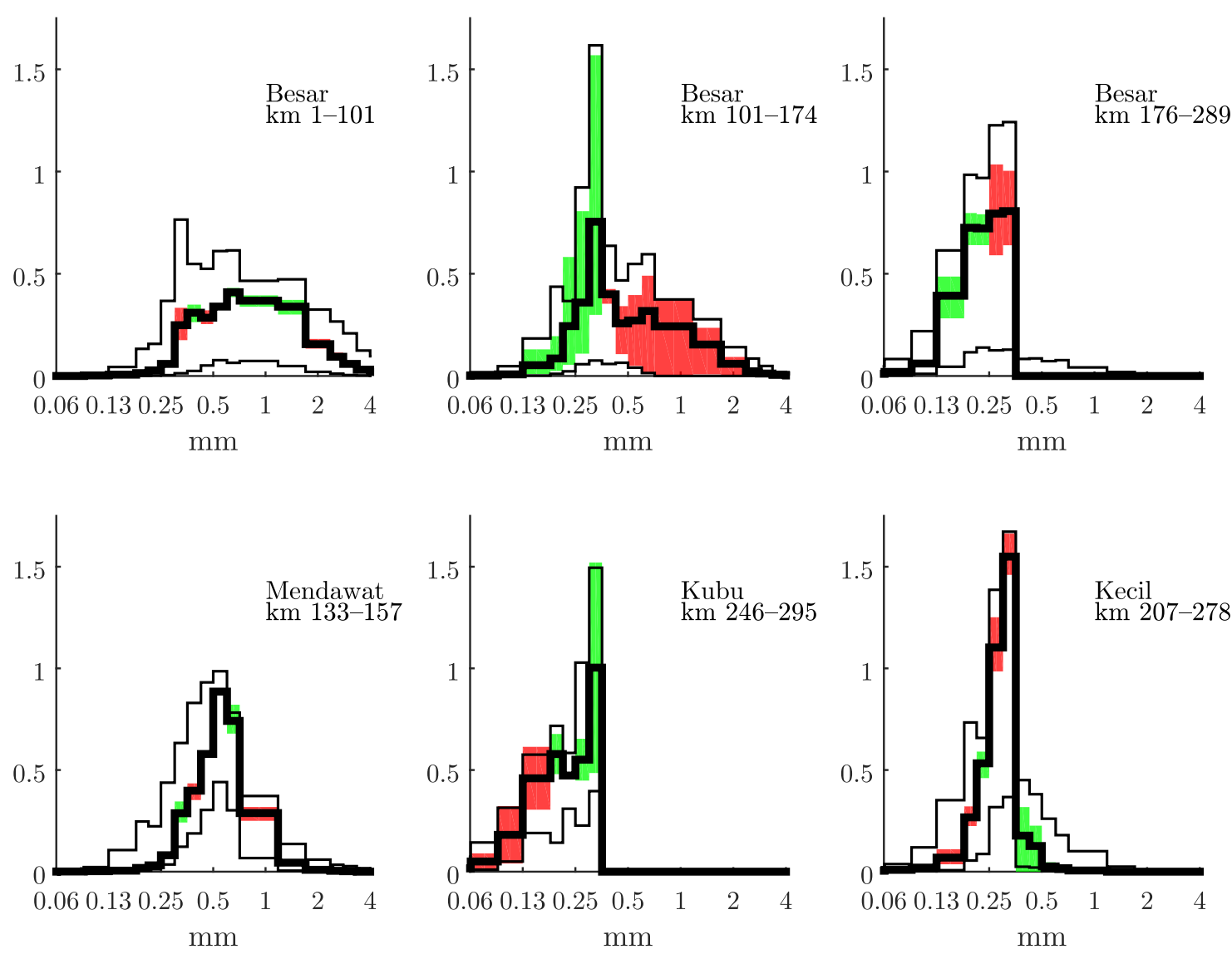

Figure 3.8: Histograms of the grain size distribution by reach; bars indicate the $16^{\text {th }}, 50^{\text {th }}$ and $84^{\text {th }}$ percentile of each bin; and green areas indicate gains and red area indicate losses in downstream direction

Consistent with downstream fining in the main distributary, the initial grain sizes in the side distributaries directly after branching off are lower when the bifurcation is located further downstream. Thus, the grain size is coarsest in the Mendawat and finest in the Kubu. At the bifurcations, there is only a small difference between grain sizes in the side distributaries and in the main channel. The median grain size in the Kapuas Kecil is slightly coarser, and bed material in the Kubu channel is slightly finer than that of the Kapuas Besar (Figure 3.6a). The most striking downstream trend difference between the side distributaries and the main distributary is seen in the development of grain sizes. The material in the Kapuas Kecil and Kubu distributaries coarsens in downstream direction. The trend of coarsening is stronger in the Kubu distributary than in the Kapuas Kecil. Coarsening in both distributaries is a consequence of winnowing of fines (Figure 3.8). The grain size trend was not 
a)

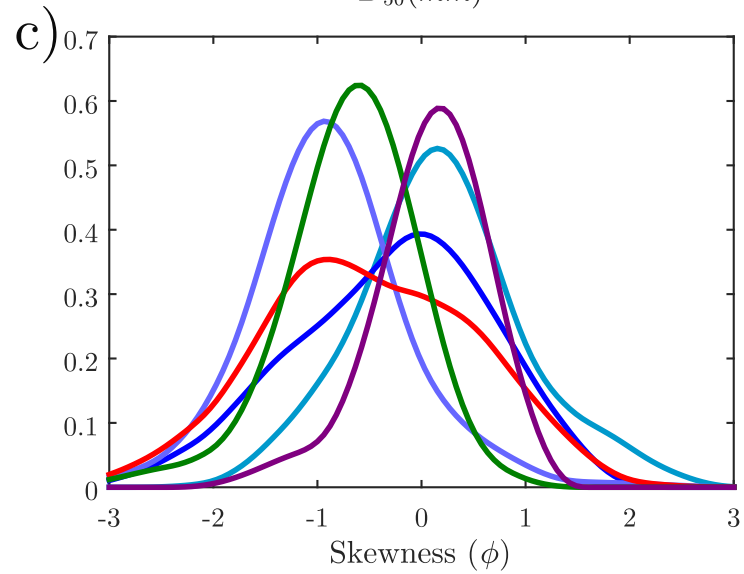

b)

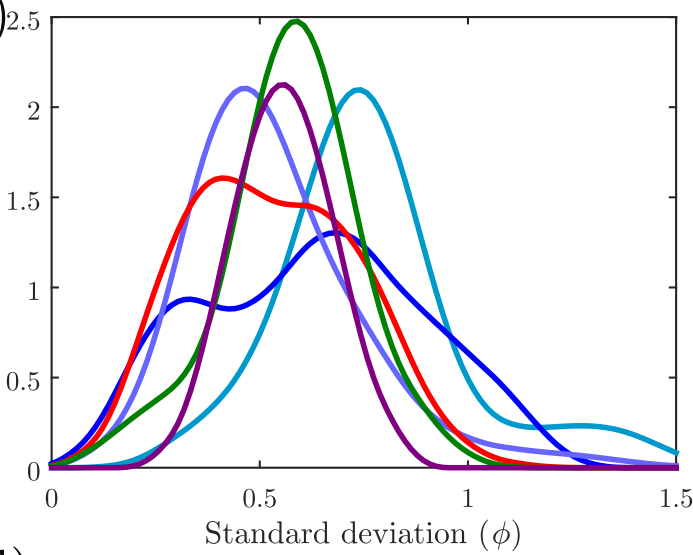

d)

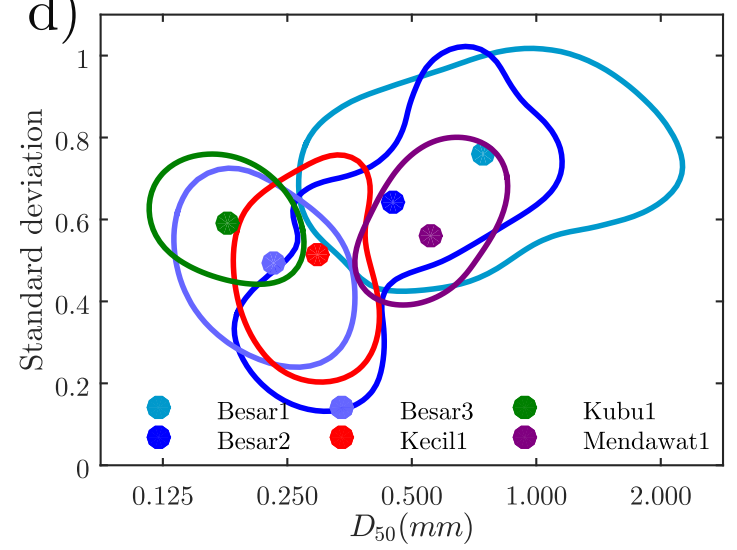

Figure 3.9: Distribution of a) the median, b) the standard deviation and c) the skewness among the samples grouped by reach as well as d) the joint distribution of median and standard distribution; The contour lines contain $68 \%$ of the samples of each reach.

established for the Mendawat, because the surveyed section was insufficiently long. The skewness of the grain size distribution caused by the lack of grains larger $0.35 \mathrm{~mm}$ extends from the Kapuas Besar into the Kapuas Kecil and Kubu distributaries. However, while coarse material is completely absent in the Kubu distributary, the fraction of interspersed coarse material in the Kapuas Kecil is larger than in the Kapuas Besar. The Kubu distributary has no material larger than the mode of the distribution, contrary to the Kapuas Kecil case. This causes the downstream trends of standard deviation and skewness to be different in both distributaries, although they both coarsen, due to winnowing of fines (Figure 3.6). The standard deviation of the grain size distribution of the Kubu distributary is reduced, whereas it increases in the Kapuas Kecil. Similarly, the grain size distribution becomes more skewed towards coarser material in the Kubu distributary, while it becomes less skewed in the Kapuas 


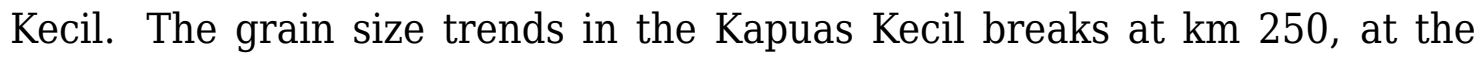
confluence with the Landak tributary.

\subsection{Discussion}

\subsubsection{Comparison with the Mahakam Delta}

Rodríguez-Iturbe and Rinaldo (2001) used an aerial photograph of the Kapuas alluvial plain to illustrate the complexity of delta channel networks. Following Galloway's classification (Galloway, 1975), the Kapuas delta is a mixed rivertide dominated delta, similar to the Mahakam Delta previously analyzed by Sassi et al. (2012). Both these deltas on Borneo drain a similar catchment and are subject to similar forcing but show essential differences in their planforms. The Mahakam forms a single channel before branching into a large fan-shaped delta. The Kapuas River system has several distributaries branching off further upstream and only a small fan-shaped delta. In the Mahakam Delta, sinuous tidal channels can clearly be distinguished from nearly rectilinear fluvial distributaries (Figure 3.1). Such tidal creeks are absent in the Kapuas plain. The differences between the systems may relate to the geological constraints fixing the Mahakam River up until the delta apex, inhibiting avulsion and the formation of side distributaries (Chambers et al., 2004). Yet both rivers show an along-channel break of geometric scaling at a point where tidal influence becomes stronger than the river influence. Downstream of the break in the Kapuas, the increasing tidal influence is apparent from the gradual decrease of channel curvature towards the sea, which is similar to tidal meanders (Marani et al., 2002). The large trifurcation in the Kapuas delta-fan can be seen as an additional indication of a strong tidal influence in this reach (Leonardi et al., 2013).

\subsubsection{Comparison with idealized model results}

A comparison of the present topography of the Kapuas channel network with a historic map (Hydrograaf, 1907) shows that no substantial planform changes have occurred since 1882, suggesting that the river can be considered to be close to a dynamic morphodynamic equilibrium. This makes the Kapuas suitable for comparison with equilibrium river profiles. In the Kapuas, the width convergence breaks at the delta apex, which has implications for tidal propagation and sediment transport. The width reduction and depth increase are such that the cross section area remains remarkably constant, especially in the Kapuas Besar. This is less so in the Kapuas Kecil, but the latter channel 
is subject to dredging. Idealized models of tidal channels often impose a fixed width that is continuously converging (Seminara et al., 2012; Bolla Pittaluga et al., 2014, 2015b). For a model setup with a fixed width and constant discharge adopted by Bolla Pittaluga et al. (2014), equilibrium bed level profiles showed an adverse bed slope over a small section near the mouth bar region, which corresponds to a depth increase. Results from the Kapuas show the depth increase continues up to the point where width no longer converges, which is at a distance from the mouth that is small compared to the length over which the tide attenuates. The constancy of the cross section area along the channels suggests that the aspect ratio is a key variable to be resolved in theoretical studies on equilibrium channel geometries.

\subsubsection{Comparison with hydraulic geometry relations}

All Kapuas distributaries consist of a fluvial reach terminating in a tidal funnel. Comparison of the fluvial reaches to empirical hydraulic relationships allows to determine how similar the distributaries are to fluvial channels. Based on an elaborate review, Eaton (2013) introduced the following relations:

$$
\begin{gathered}
Q_{\mathrm{bf}}=(w / 3.35)^{(1 / 0.536)} \\
Q_{\mathrm{bf}}=(h / 0.305)^{(1 / 0.384)}
\end{gathered}
$$

The ratio of discharges based on width and based on depth (respectively), $r$, then reads as

$$
r=\frac{(w / 3.35)^{(1 / 0.536)}}{(h / 0.305)^{(1 / 0.384)}} .
$$

The expected value of $r$ is 1 and does not depend on the channel size. $r>1$ indicates an excess of width over depth, compared to alluvial channels without tides. Figure 3.10 shows the nonlinear width-to-depth ratio of the Kapuas distributaries. The width-to-depth ratio is larger in the upstream than in the downstream half of the alluvial plain. Upstream of the tidal funnels, the ratios of $r$ are smaller than one, which renders the river comparatively deep. The tides systematically adjust the aspect ratio far beyond the value characteristic for alluvial rivers, which can be seen as tidal channels having a depth excess.

\subsubsection{Influence of bifurcation geometry on sediment division}

The similarity of bed material in the branches of the distributaries indicates that the transport of bed material into the side distributaries is inhibited neither by the particular u-shape of the bifurcations nor by the increase in bed 


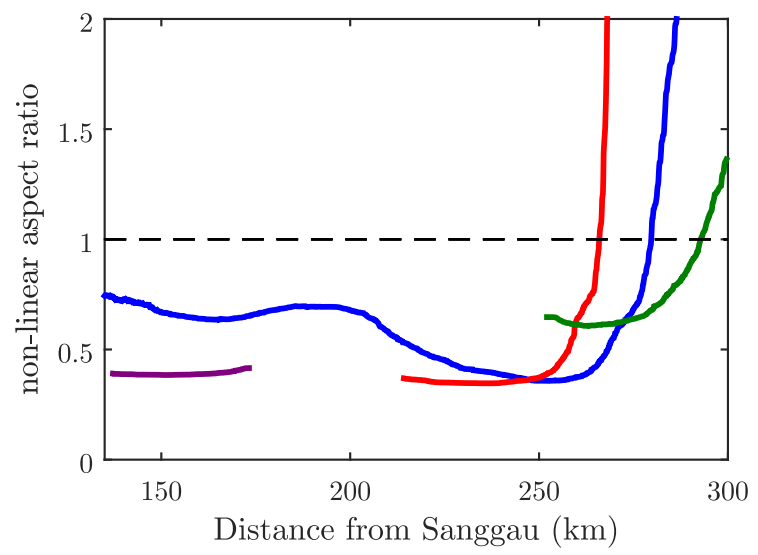

Figure 3.10: Non-linear channel aspect ratio derived from empirical hydraulic geometry relationships. All distributaries are are narrower and deeper in their upstream part then expected from the empirical relationship.

level. Suspended load is typically the dominant mode of transport in meandering rivers, such as the Kapuas (Schumm, 1985). The shape of the bifurcations is similar to the geometry of tie channel junctions connecting rivers to lakes (Rowland and Dietrich, 2005). Unlike tie channels, the side distributaries of the Kapuas receive sufficient sediment to retain an active bed. The large size and dense vegetation on the spits separating the side channel from the main river further indicate the long-term stability of the bifurcation and the role of cohesive banks in planform development. This particular topography is rare but can be observed in other rivers on Borneo and in the Niger Delta (Abam, 1999).

The bed material grain size is often discontinuous at river bifurcations, such as observed in the Rhine (Frings and Kleinhans, 2008b). At bifurcations with different bed level between the branches, the shallower branch receives fewer and finer material, as both the grain size and the concentration of suspended sediment decrease with vertical distance to the bed. The winnowing of fines in the Kubu and Kapuas Kecil confirms a low sediment supply to those channels. However, both in the Kapuas Kecil and in the Mendawat channel, the bed material is not finer than upstream of the respective bifurcation, only material in the Kubu branch is finer. Bend sorting can also contribute to differences of bed material in bifurcation branches. Branches located in outer bends receive fewer and coarser material (Ikeda et al., 1987; Frings, 2008). The trend of bed material size in the main branch is not discontinuous across the Kapuas bifurcation. The limited influence of the bifurcations on the Kapuas Besar can be attributed to the small size of the side distributaries. The grain size in the 
Kapuas Kecil and the Mendawat channels, both branching off in outer bends, is only slightly larger than in the corresponding section of the main channel. The grain size in the Kubu at its inlet is even finer than in the corresponding section of the Kapuas Besar channel, although the Kapuas Besar is deeper and has coarser bed material at the inlet of Kubu. Thus, bend sorting may be less important in suspended load rivers. Other factors than bifurcation geometry, such as the phase difference of the tidal wave between the channels, may have an important influence on the division of sediment at bifurcations.

\subsubsection{Sediment transport regime}

The rapid decrease of the median grain size in the reach between $\mathrm{km} 110$ and 150 is caused by selective deposition of material larger than $0.35 \mathrm{~mm}$. Within this reach there is a strong reduction of the bankfull water surface slope (Figure 3.3a), as the river transits from the upstream valley to the alluvial plain (Figure 3.2). The reduction of grain size and widening of the river compensate for the reduction of the slope to maintain constant sediment transport. Frings (2008) gives the distance over which the grain size halves for several large sand bed rivers. Figure 3.11 compares the rate of fining and decay length to the base $e$. The rate of downstream fining in the alluvial plain of the Kapuas is large compared to other rivers. The alluvial plain of the Kapuas is shorter than of those of the rivers used for comparison, which may contribute to a more rapid reduction of the grain size. Downstream of $\mathrm{km} \mathrm{150,} \mathrm{all} \mathrm{remaining}$ grain size fractions are transported and the rate of fining decreases. The continuation of downstream fining towards the river mouth suggests that tides have limited impact on the sediment sorting processes in the Kapuas distributaries, as strong tidal influence causes an increase of grain size towards the river mouth (Dalrymple and Choi, 2007).

\subsubsection{Stability of the distributary channel network}

Slingerland and Smith (1998) developed a mathematical model for the initiation of river avulsion. They found that grain size influences the probability that following an avulsion both channels remain permanently open. In their model, avulsion was followed by quick abandonment of either of the two branches for grains of $0.1 \mathrm{~mm}$, while for grain sizes of $0.4 \mathrm{~mm}$, both branches remained open, provided that the slope advantage of the new branch remains below a factor of 5. Median grain size in the Kapuas remains above $0.2 \mathrm{~mm}$, suggesting that partial avulsions resulting in two stable branches are likely. Stable bifurcations obtain an asymmetric configuration, where one branch is larger 


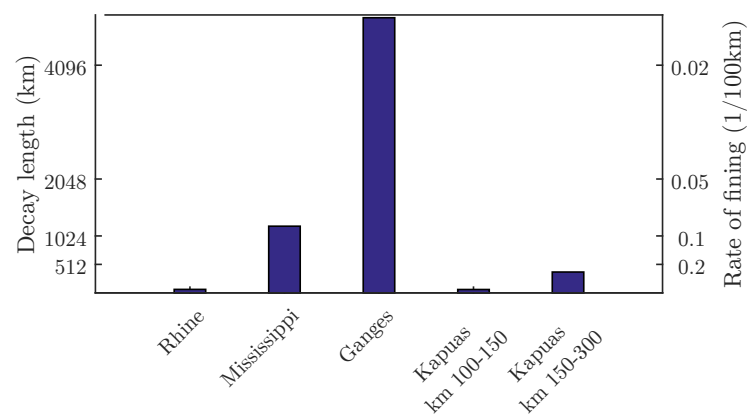

Figure 3.11: Comparison of downstream fining of bed material between the Kapuas and other large sand bed rivers, adopted from Frings (2008), and original data from ten Brinke (1997); Nordin and Queen (1992); Singh et al. (2007)

than the other (Wang et al., 1995; Bolla Pittaluga et al., 2003; Edmonds and Slingerland, 2008; Bolla Pittaluga et al., 2015a). The Kapuas is no exception to this.

The increase of grain size due to winnowing of fines along the Kapuas Kecil and Kubu indicate that the sediment transport is directed seaward and that those channels are not aggrading. This corresponds to the observation that the side channels do not show any sign of infilling compared to the survey in 1882. This is in contrast to three small avulsion loops located in the upstream part of the alluvial plain that are not directly connected to the sea and have partially filled in since then (Figure 3.12). The morphological stability of channels in the Kapuas thus depends on whether channels reconnect before reaching the sea or not.

\subsection{Conclusion}

The Kapuas River forms a complex, natural channel network, consisting of one main stream and three side distributaries. Tides in the Kapuas strongly depend on discharge, ranging from nearly complete admittance during low flow to complete attenuation during high flow, towards the upstream end of the alluvial plain. This makes the Kapuas ideal to investigate channel geometry and bed material grain size in the fluvial-tidal transition zone. All distributaries of the Kapuas consist of a long and deep fluvial upstream reach and a short and shallow tidal funnel at the downstream end, which terminate in shallow mouth bars. In each distributary there is a clear break of channel geometrical properties between these two reaches, at which the channel width obtains a minimum and channel depth a maximum. Thus the Kapuas distributaries are neither continuously converging, as in estuarine channels, nor non-converging 


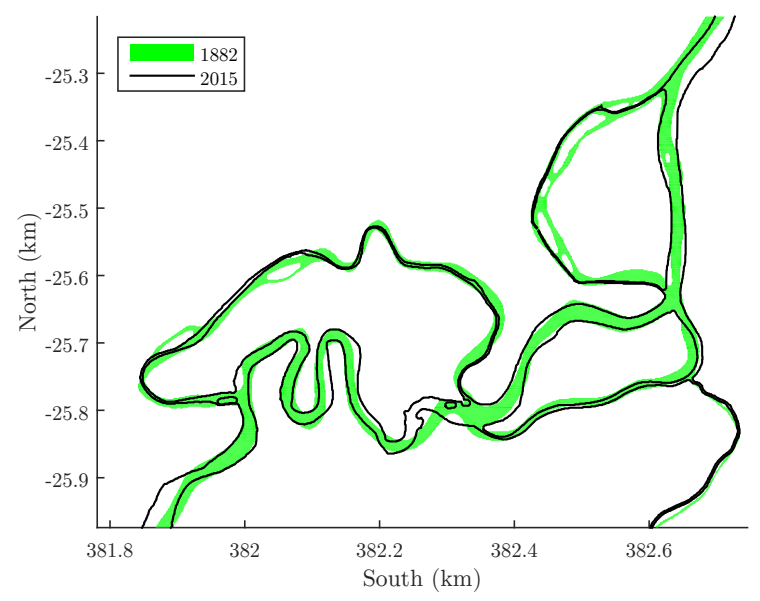

Figure 3.12: Three avulsion loops in the Kapuas located between $\mathrm{km} 125$ and 165 downstream of Sanggau, in 1882 and 2015; in all loops one branch has been filled in, in strong contrast to distributaries connected to the sea, which do not have considerably changed their width over the same time span.

as in delta channels unaffected by tides. The Mahakam delta shows a similar break of channel geometry, indicating that this phenomenon may be a general characteristic of the fluvial-tidal transition. Trends of bed material grain size in the Kapuas were found not to be related to the channel geometry. Bed material in the main distributary shows a downstream fining trend, similar to that typically observed in rivers. There is no corresponding change of the grain size properties where the trend of downstream channel geometry breaks. Bed material in the side distributaries becomes coarser in the downstream direction, in contrast to the downstream fining in the main distributary. Differences in grain sizes between downstream branches may be caused by undersupply of sediment to the side distributaries, related to the asymmetric shape of bifurcations. Thus, in contrast to channel geometry, bed material grain size does strongly depend on the division of sediment at bifurcations.

\section{Acknowledgement}

This research was supported by the Royal Netherlands Academy of Arts and Sciences (KNAW), project SPIN3-JRP-29. The authors thank Hidayat for the processing of bed material samples at the Indonesian Institute of Sciences, as well as Muhammad Pramulya (Tanjungpura University), Laura Schlebes and Judit Snethlage (both Wageningen University) for their field work support. We also thank Pieter Hazenberg (Wagening University) for technical support. We also thank the Editor, Giovanni Coco, and three anonymous reviewers for 
giving constructive feedback to the draft version of this manuscript. Preprocessed data, including a detailed description thereof, are provided as supporting information (SI) files. Bulk raw data are available from the first author (Wageningen University, Hydrology and Quantitative Water Management Group, P.O. box 47, 6700 AA Wageningen, The Netherlands, karl.kastner@wur.nl). 


\section{$4 \mid \begin{aligned} & \text { Propagation of tides along a river } \\ & \text { with a sloping bed }\end{aligned}$}

Conceptually tidal rivers are seen as narrow channels along which the cross section geometry remains constant and the bed is horizontal. As tidal waves propagate along such a channel, they decrease exponentially in height. The decrease is the more rapid the stronger river flow. Near the coast, the tidally averaged width and depth change little throughout the year, even if the river discharge varies strongly between the seasons. However, further upstream, the water depth varies considerably with the river discharge. Recent observations from the Kapuas River, Indonesia, show that the water surface forms a backwater profile when the river flow is low. In this case, the depth converges, i.e. it gradually decreases between the river mouth and the point where the bed reaches sea level. This distinctly influences how tidal waves propagate up-river so that their wave height does not decrease exponentially any more. We present a theoretical analysis of this phenomenon, which reveals several so far overlooked aspects of river tides. These are in particular relevant for low river flow. Along the downstream part of the tidal river, depthconvergence counteracts frictional damping so that the tidal range is higher than expected. Along the upstream parts of the tidal river, the low depth increases the damping so that the tide more rapidly attenuates. The point where the bed reaches sea level effectively limits the tidal intrusion. This carries over to the overtide and the subtidal water level setup.

This chapter is based on: Kästner, K., and A. J. F. Hoitink, Effects of a sloping bed on the propagation of tides up-river, Submitted to Journal of Fluid Mechanics, 2019d 


\section{Symbols}

\begin{tabular}{|c|c|c|}
\hline Quantity & Unit & Description \\
\hline$\alpha$ & 1 & relative strength of river and tidal flow \\
\hline$\omega_{j}$ & $1 / \mathrm{s}$ & angular frequency of jth-tidal species \\
\hline$\Delta \varphi_{1}$ & 1 & phase difference between surface elevation and velocity \\
\hline$\varphi_{j z}, \varphi_{j u}$ & 1 & phase of the j-th frequency component of $z_{j}$ and $u_{j}$ \\
\hline$a$ & 1 & strength of friction \\
\hline$A$ & $\mathrm{~m}^{2}$ & wetted cross-sectional area \\
\hline$c_{0}, c_{1}, c_{2}$ & 1 & coefficients of the characteristic polynomial \\
\hline$c_{d}$ & 1 & drag coefficient \\
\hline$f_{0}, f_{1}, f_{2}$ & 1 & Chebychev coefficients of the friction term \\
\hline$F$ & $\mathrm{~m}^{6} / \mathrm{s}^{2}$ & Chebychev approximation of the signed square $Q|Q|$ \\
\hline$F_{j}, F_{j}^{\prime}$ & $\mathrm{m}^{6} / \mathrm{s}^{2}$ & jth-frequency component of $\mathrm{F}$, time derivative thereof \\
\hline$g$ & $\mathrm{~m} / \mathrm{s}^{2}$ & acceleration by gravity \\
\hline$h$ & $\mathrm{~m}$ & water depth \\
\hline$h_{0}$ & $\mathrm{~m}$ & tidally averaged water depth \\
\hline$h_{u}$ & $\mathrm{~m}$ & asymptotic uniform flow depth upstream \\
\hline $\mathrm{i}$ & 1 & imaginary number \\
\hline$k_{1}$ & $1 / \mathrm{m}$ & wavenumber with friction and convergence \\
\hline$k_{1 z}, k_{1 Q}$ & $1 / \mathrm{m}$ & wavenumber of the surface elevation and discharge \\
\hline$k_{1,0}$ & $1 / \mathrm{m}$ & wavenumber with neither friction nor convergence \\
\hline$k_{1, a}$ & $1 / \mathrm{m}$ & wavenumber with friction but no convergence \\
\hline$\Delta k_{1}$ & $1 / \mathrm{m}$ & change in wavenumber introduced by convergence \\
\hline$Q, Q_{j}$ & $\mathrm{~m}^{3} / \mathrm{s}$ & discharge, frequency components thereof \\
\hline$Q_{0}$ & $\mathrm{~m}^{3} / \mathrm{s}$ & river discharge \\
\hline$Q_{n}$ & $\mathrm{~m}^{3} / \mathrm{s}$ & river discharge, for which tidally averaged flow is uniform along river \\
\hline$Q_{h r}$ & $\mathrm{~m}^{3} / \mathrm{s}$ & half the tidal discharge range \\
\hline$R^{ \pm}$ & $1 / \mathrm{m}$ & coefficient of reflection \\
\hline$r^{ \pm}$ & $1 / \mathrm{m}$ & roots of the characteristic polynomial \\
\hline$r_{0}$ & $1 / \mathrm{m}$ & damping coefficient of the subtidal harmonic \\
\hline$r_{1}$ & $1 / \mathrm{m}$ & damping coefficient of the main tidal species \\
\hline$r_{2}$ & $1 / \mathrm{m}$ & damping coefficient of the first even overtide \\
\hline$R_{h}$ & $\mathrm{~m}$ & hydraulic radius \\
\hline$T_{1}$ & $\mathrm{~s}$ & period of the main tidal species \\
\hline$t$ & $\mathrm{~s}$ & time \\
\hline$T^{ \pm}$ & $1 / \mathrm{m}$ & coefficient of transmission \\
\hline$u, u_{j}$ & $\mathrm{~m} / \mathrm{s}$ & velocity, j-th frequency component thereof \\
\hline $\mathrm{w}$ & $\mathrm{m}$ & river width \\
\hline$x$ & $\mathrm{~m}$ & distance from the river mouth \\
\hline$z_{0}$ & $\mathrm{~m}$ & tidally averaged water level \\
\hline$z_{0}^{\prime}$ & $\mathrm{m}$ & subtidal water level offset \\
\hline$z_{b}$ & $\mathrm{~m}$ & bed elevation with respect to datum \\
\hline$z_{s}, z_{j}$ & $\mathrm{~m}$ & surface elevation, jth-frequency component thereof \\
\hline
\end{tabular}




\subsection{Introduction}

The tide in the ocean can readily be predicted, as it constitutes are a direct response to the harmonic movement of the celestial bodies (Foreman, 1996; Ray et al., 2011). Unlike ocean tides, tides in rivers are modulated by variable rainfall runoff (Hoitink and Jay, 2016). While the tide propagates up-river, its amplitude and phase are modified by changes of the cross section geometry (Green, 1838) as well as by friction (Lorentz, 1926; Ippen, 1966). A decrease of the cross section area raises the amplitudes of surface elevation and velocity, while friction has the opposite effect (Jay, 1991; Savenije et al., 2008). Eventually, far upstream, friction prevails and the tidal wave diminishes, the more rapidly the stronger the river flow (LeBlond, 1978; Godin, 1985). The cross section geometry is conventionally considered to be constant (Savenije et al., 2008), with the exception of tidal flats in some studies (e.g. Friedrichs and Madsen, 1992). While this assumption holds for strongly width-converging estuaries, it is inappropriate for long rivers with little variation of width. Here, the sloping river bed (Seminara et al., 2012), as well as the seasonal variation of river discharge (Dai and Trenberth, 2002) lead to strongly different backwater profiles throughout the year. Based on a theoretical analysis, this contribution explains why backwater dynamics cause the tide to propagate very differently between periods of high and low river flow. During high flow, the tidal range decreases exponentially along the channel. During low flow, the convergence of the depth into the upstream direction causes the tidal range to decrease less rapidly along the downstream part of the tidal river while the shallow depth causes the tidal range to decrease more rapidly along the upstream part of the tidal river.

River tides are described by the non-linear shallow-water equations, which, in general, do not admit a closed-form solution. Theoretical insight into river tides, therefore, builds on simplifications of the underlying equations, as well as a reduced complexity of the river geometry. We focus here on tidal rivers where seasonally averaged, the net discharge of the river is stronger than that of the tide, so that the flow does not reverse, and where the water remains fresh (Godin, 1985). Tidal rivers often form long channels of nearly constant width that are connected to the sea by a short, width-converging reach, the tidal funnel (figure 4.1). The tide travels up the river at a length that exceeds many times the length of the funnel. This sets tidal rivers apart from tidally dominated estuaries that strongly converge in width along their entire length and have brackish water (Pritchard, 1967). The bed of long non-converging estuaries is typically horizontal (Savenije, 2015), except at the mouth, where 
there are shallow sandbars. Idealized models thus represent tidal rivers as non-converging channels with a horizontal bed, along which width and depth remain constant (Godin, 1985, 1991b).

The dependence of the water depth on the river discharge is commonly ignored in models of tidal propagation (Godin, 1984; Horrevoets et al., 2004; Savenije et al., 2008). This allows for an analytic solution of the propagation of tidal waves upriver with an amplitude that is much smaller than the water depth (Godin, 1991b). If there is no river flow, then the tide is gradually damped and delayed proportionally to the tidal amplitude and the amplitude decreases exponentially along the channel (Ippen, 1966; Friedrichs, 2010). River discharge superimposes a mean flow velocity, which increases friction. The tidal amplitude still decreases exponentially when the river flow is strong, but at a higher rate that is proportional to the square root of the mean flow velocity (LeBlond, 1978; Godin, 1985, 1991b; Jay, 1991; Jay and Flinchem, 1997; Godin, 1999; Alebregtse and de Swart, 2016). Even the few models that do consider the water level set-up, neglect the slope of the bed (Cai et al., 2014). However, the bed of tidal rivers typically slopes up beyond the upstream end of tidal funnels (Seminara et al., 2012) and (chapter 3). It is well-known that the rising riverbed limits the tidal intrusion approximately to the point where the bed reaches sea level (Dalrymple et al., 2015; Nienhuis et al., 2018). Here, we demonstrate that this is not because the waves cannot run up the slope, but rather because friction is always strong in the upstream part of the tidal river. The sloping bed causes the tidally averaged water depth to gradually vary along the river, except for periods where the river is at normal flow so that the water surface slope is identical to the bed slope (figure 4.1). The depth can thus converge over a long distance, even though the width may only converge along the short tidal funnel. This contribution explores the implications of systematic depth variations.

Our study is motivated by observations in the Kapuas River, Indonesia, which features a seasonal backwater variation that strongly influences tidal propagation. These observations are not well predicted by conventional models that do not take the backwater effect into account. This paper extends the conceptual understanding of river tides by providing a theoretical model that explains how the tide propagates along a backwater affected river, such as the Kapuas. Section 2 presents observations of the tide and backwater variation in the Kapuas River. Section 3 develops a general theory of river tides, following the classical approach by transforming the shallow water equations into the wave equation (Lamb, 1932; Dronkers, 1964; Ippen, 1966; Parker, 1984). We show that the propagation of the tide along a channel with varying ge- 

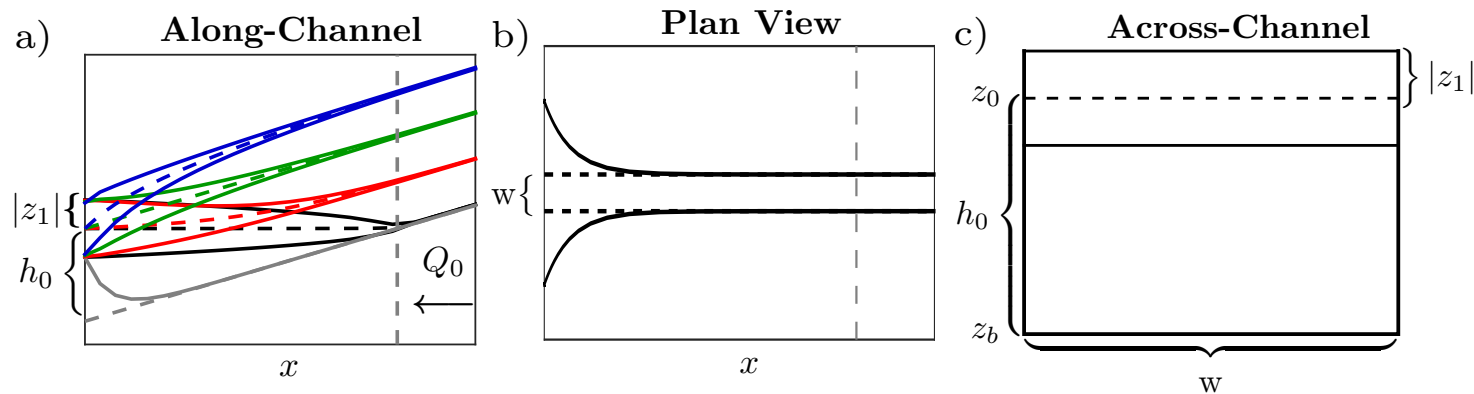

Figure 4.1: Idealized geometry of a tidal river: The width w and bed slope $\frac{\partial z_{b}}{\partial x}$ remain constant along the river, except for the short funnel-shaped section that connects the river to the sea. The tidally averaged surface elevation $z_{0}$ (dashed) depends on the river discharge $Q_{0}$. It forms a backwater profile (black) when the river discharge is low and a drawdown curve when the river discharge is high (blue). Both the tidally averaged depth $h_{0}$ and tidal amplitude $\left|z_{1}\right|$ gradually vary along channel depending on the river discharge. For normal flow $\left(Q_{0}=Q_{n}\right)$ (green), the tidally averaged depth remains constant along the river $\left(\frac{\partial h}{\partial x}=\frac{\partial z_{s}}{\partial x}-\frac{\partial z_{b}}{\partial x}=0\right)$.

ometry can be interpreted as the transmission and reflection at a sequence of infinitesimal steps. This analogy is used to determine the damping and celerity of the tidal wave along a channel with gradually varying cross section geometry. Based on the theory developed in Section 3, Section 4 shows how the tide propagates along a river with a sloping bed. Section 5 discusses the main results and in Section 6, conclusions are drawn.

\subsection{Tidal propagation along the Kapuas River}

The Kapuas River is located in West Kalimantan, Indonesia (figure 4.2). The catchment is situated in the humid tropics so that the river discharge varies strongly with the monsoon (chapter 2). The bed of the Kapuas is moderately sloping (chapter 3). These conditions result in different backwater profiles between the wet and dry season, which in turn strongly affect the propagation of the tide. The Kapuas River has one large distributary, from which three smaller distributaries branch off. The smaller distributaries only slightly affect the tide in the main stem of the river. Due to the microtidal regime, the distributaries only funnel along a short reach close to the sea. This renders the Kapuas an ideal case to study the propagation of tides along a backwater affected tidal river.

The tidally averaged water surface forms a pronounced backwater profile during low flow but remains nearly parallel to the riverbed during high flow 


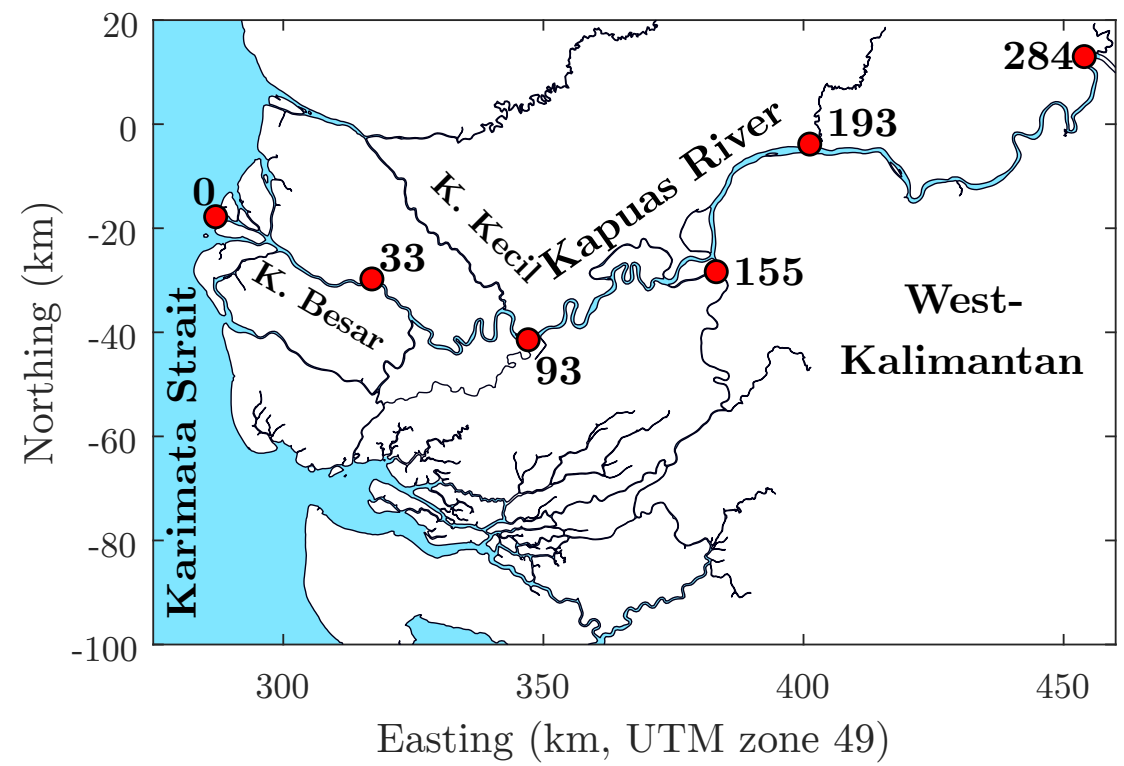

Figure 4.2: Coastal zone of the Kapuas River, selected gauging stations are labelled with their respective distance to the river mouth.

(figure 4.4a). The tidally averaged water level increases with the river discharge, the further a station is located from the sea. The tidally averaged water level ranges over $10 \mathrm{~m}$ at Sanggau, $285 \mathrm{~km}$ from the sea, but only by $2 \mathrm{~m}$ at Mendawat, $130 \mathrm{~km}$ from the sea. The tidal range decreases with distance from the coast, and with river discharge (figures 4.3 and 4.4b). At high flow, the damping is nearly exponential, and the tidal range drops to half the initial value at $\mathrm{km} \mathrm{50}$. For lower discharges, the admittance, i.e. the ratio of the tidal surface elevation amplitude along the river and the amplitude at the river mouth, is higher. During low flow, the shape of the admittance along the river is very different from that of a decaying exponential. Close to $\mathrm{km} \mathrm{150,}$ the admittance has a knickpoint, where the damping strongly increases. Up to this point, the tidal amplitude is isosynchronous, i.e. remains constant during low flow. Below a river discharge of $5000 \mathrm{~m}^{3} / \mathrm{s}$, the tide becomes noticeable at Sanggau. At extreme low flow, the tidal range at Sanggau is still half as large as the range at sea. Conventional tidal models that do not include the backwater effect predict that the tidal admittance decreases exponentially with increasing distance from the sea, and thus fail to explain the observed isosynchronous admittance during low flow. The following section extends the theory of river tides by variable backwater effects, which predicts the tide in agreement with the observation. 

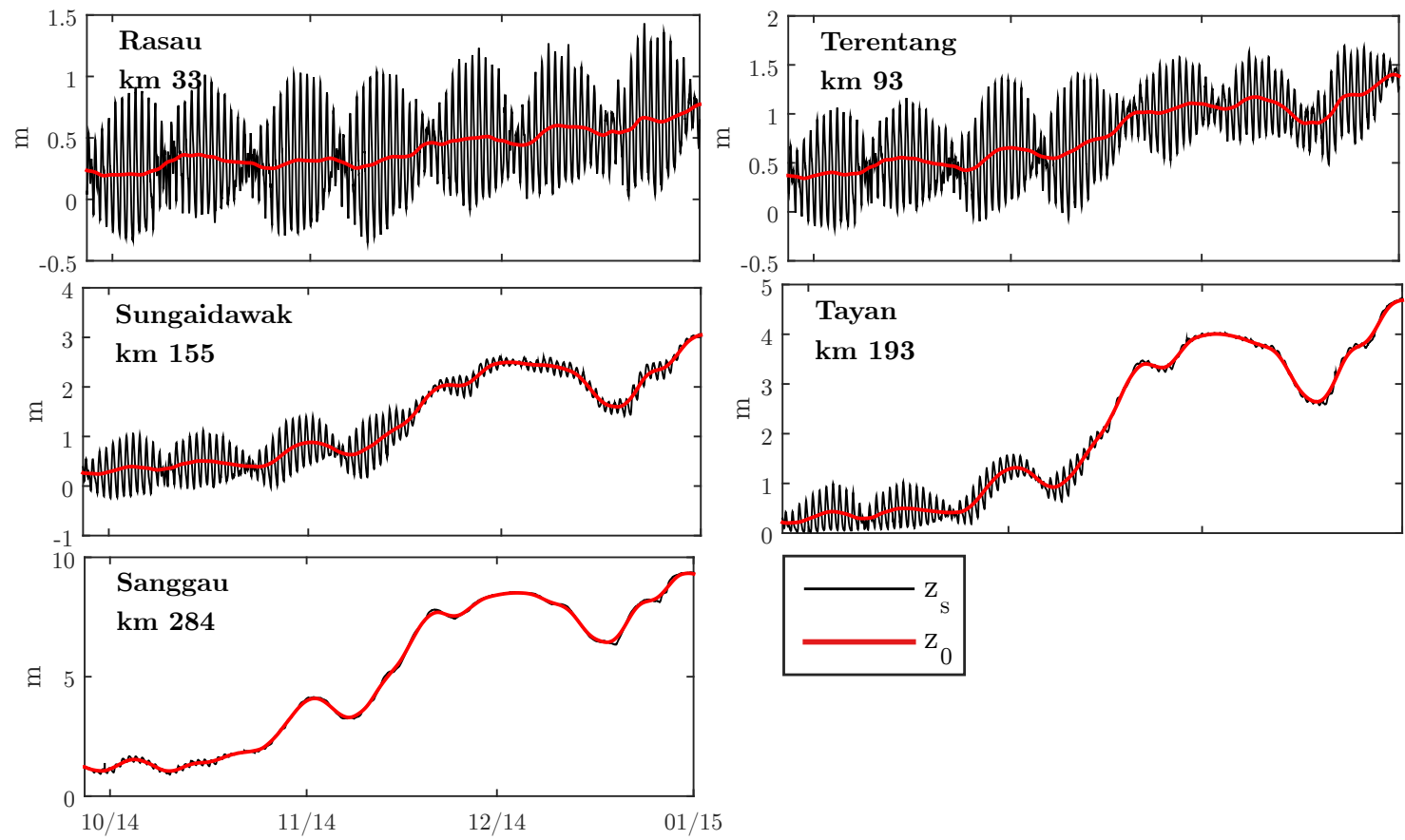

Figure 4.3: Time-series of the surface elevation $z_{s}$ (black) and its tidal average $z_{0}$ (red) at five gauging stations along the Kapuas River. $z_{0}$ is determined by low pass filtering with a cut-off period of one tidal cycle so that the subtidal variation over the spring-neap cycle remains.
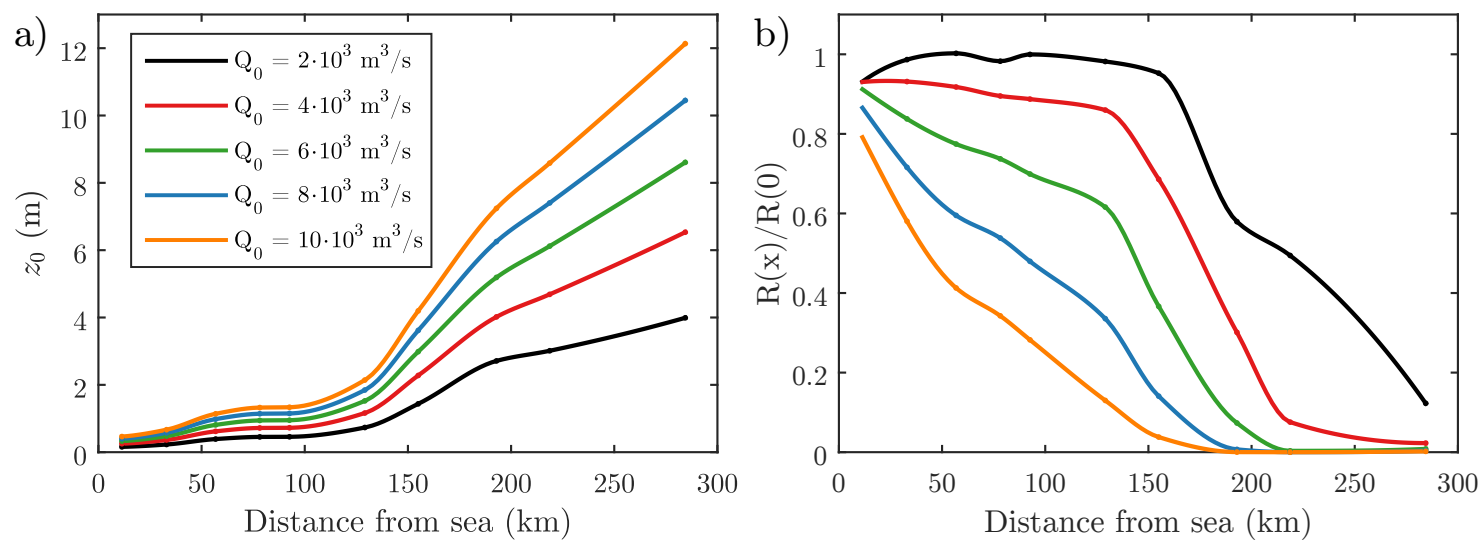

Figure 4.4: a) Observed tidally averaged water level and b) Admittance of tidal range along the Kapuas River at different river discharges 


\subsection{Generic model of river tides}

\subsubsection{Tidal waves}

The tide causes the water surface elevation $\mathrm{z}_{\mathrm{s}}$ and discharge $\mathrm{Q}$ to periodically oscillate over time $t$, which suggests to separate them into the components $\mathrm{z}_{\mathrm{j}}=\operatorname{Re}\left\{z_{j}\right\}$ and $\mathrm{Q}_{\mathrm{j}}=\operatorname{Re}\left\{Q_{j}\right\}$ with frequencies $\omega_{j}$ (Godin, 1991c):

$$
\begin{aligned}
& \mathrm{z}_{\mathrm{s}}(t, x)=\sum_{j=0}^{\infty} \mathrm{z}_{\mathrm{j}}(t, x)=\mathrm{z}_{0}(x)+\sum_{j=1}^{\infty} \operatorname{Re}\left\{z_{j}(x) \exp \left(\mathrm{i} \omega_{j} t\right)\right\} \\
& \mathrm{Q}(t, x)=\sum_{j=0}^{\infty} \mathrm{Q}(t, x)=\mathrm{Q}_{0}+\sum_{j=1}^{\infty} \operatorname{Re}\left\{Q_{j}(x) \exp \left(\mathrm{i} \omega_{j} t\right)\right\}
\end{aligned}
$$

For brevity, the explicit dependence on $x$ is omitted from the notation further on. The subscript $j$ denotes the frequency. The frequency components are determined by the inner product $(y)_{j}=\frac{1}{T} \int_{T} y\left(\cos \omega_{j} t+i \sin \omega_{j} t\right) \mathrm{d} t$, where the time $T$ is the least common multiple of all periods.

The surface elevation of each frequency component has a distinct amplitude $\left|z_{j}\right|$ and phase $\varphi_{j z}=\arctan \left(\operatorname{Im}\left(z_{j}\right) / \operatorname{Re}\left\{z_{j}\right\}\right)$. The astronomical tide consists of an infinite number of constituents (Pugh, 1987). Their frequencies are integer combinations of basic frequencies derived from the orbits of the celestial bodies (Doodson, 1921b; Cartwright and Tayler, 1971; Souchay et al., 2012). Several dozen constituents are required to accurately predict ocean tides, of which many constituents are of similar frequency and magnitude. Long time series are required to separate these constituents from each other.

River discharge does not only determine the means $z_{0}$ and $Q_{0}\left(\omega_{0}=0\right)$ but also modulates the tide. River discharge varies in an irregular manner over much shorter periods than necessary for a meaningful harmonic analysis. Therefore, we consider the tide for successive periods of just one tidal cycle and decompose it into a Fourier series where the frequencies of the components are integer multiples of a single fundamental frequency, $\omega_{j}=j \omega_{1}$. The Fourier components decay rapidly in amplitude, which allows a meaningful truncation of the series to just a few components. These components are referred to as tidal species, and effectively lump tidal constituents of similar frequencies together (Kukulka and Jay, 2003b; Guo et al., 2015). Alternatively to species, the tidal wave can be interpreted as a periodic function of arbitrary shape that is described by low water and high water (Savenije, 2001; Savenije et al., 2008). This approach is supported by the observation that tidal waves travel upstream individually after each other and that the discharge of large 
rivers changes little over the time it takes for a single wave to pass. The incoming tide is thus roughly represented by a single frequency component that has an amplitude equal to half the tidal range. The range of the incoming tide changes from one tidal cycle to the next, most notably over to the spring-neap cycle. The river tide has, therefore, to be predicted for each cycle individually, depending on the incoming tide and the river flow. The amplitude and phase of a wave change as a wave propagates upriver depending on the cross section geometry and river discharge. For convenience, this is expressed in the form of the admittance $\left|z_{j}(x)\right| /\left|z_{j}(0)\right|$ and phase difference $\varphi_{j z}(x)-\varphi_{j z}(0)$, where $x$ is the distance from the river mouth. The remainder of this sections develops the theory of tidal wave propagation. It builds on previous works by Godin (1985) and Jay (1991). It advances the theory on how tides propagate along rivers with varying cross section geometry. The theory is held general and covers both mild depth and width convergence. Section 4.4 then analyses the backwater effect caused by varying river discharge and a sloping bed.

\subsubsection{Shallow water equations}

The flow in open channels is described by the one-dimensional shallow water equations (Cunge et al., 1980; Savenije, 2012b). These are the equation of continuity:

$$
\frac{\partial A}{\partial t}+\frac{\partial Q}{\partial x}=0
$$

as well as the equation of motion:

$$
\frac{\partial Q}{\partial t}+\frac{\partial}{\partial x}\left(\frac{Q^{2}}{A}\right)+\frac{1}{2} \frac{g}{\mathrm{w}} \frac{\partial A^{2}}{\partial x}=-g A \frac{\partial z_{b}}{\partial x}+g \frac{A^{2}}{\mathrm{w}^{2}} \frac{\partial \mathrm{w}}{\partial x}-c_{d} \mathrm{w} \frac{Q|Q|}{A^{2}},
$$

where $A$ is the cross-sectional area, $Q$ the discharge, w the channel width, $z_{b}$ the bed level, $g$ the gravitational acceleration and $c_{d}$ the drag coefficient. We analyze here only the case of a straight non-meandering channel that has a rectangular cross section, i.e. no intertidal areas (figure 4.1) so that the depth $h=A / \mathrm{w}$ and the surface elevation $z_{s}=z_{b}+h$. The terms on the right-hand side in (4.2b) represent the forces acting on the flow per unit distance along the channel. The forces determine how the tidal wave changes while propagating upriver. Tidal flats are not taken into account, as intertidal areas are small in rivers. The reader is referred to Jay (1991); Friedrichs and Madsen (1992); Speer and Aubrey (1985); Savenije et al. (2008) for the treatment of intertidal storage and for tidal propagation along channels of arbitrary cross sections to Li and Valle-Levinson (1999). We assume that the channel is wide 
enough so that the hydraulic radius is well approximated by the water depth and narrow enough for Rossby circulation to be relatively small. We also neglect spatiotemporal variation of the drag coefficient $c_{d}$ between high and low river flow as well as between flood and ebb flow.

\subsubsection{Wave equation}

As the tide is a periodic function, it is purposeful to decompose the shallow water equations into their frequency components. The equations are coupled by the interaction of the species due to the non-linear terms. To transform the shallow water equations into the wave equation, we consider the case where the tidal amplitude is small compared to the tidally averaged water depth $h_{0}=$ $\frac{1}{T_{1}} \int_{0}^{T_{1}} h \mathrm{~d} t . T_{1}=\frac{2 \pi}{\omega_{1}}$ is the tidal period. We neglect the small effect of nonlinearity in $1 / h$, which has been discussed in the literature (Godin, 1985). We also neglect the advective acceleration term $\frac{\partial}{\partial x}\left(\frac{Q^{2}}{A}\right)$, because its magnitude is small (Savenije, 2012b). This holds as long as $h_{0}>\left|z_{1}\right|$, which is the case as long as the bed slope is moderate or the river flow is strong.

For the mean flow $\omega_{j}=0$, continuity is trivial $\left(\frac{\partial Q_{0}}{\partial t}=\frac{\partial Q_{0}}{\partial x}=0\right)$ and the momentum equation simplifies to the backwater equation which determines the tidally averaged water level $z_{0}$ :

$$
\frac{\partial z_{0}}{\partial x}+\frac{c_{d} \mathrm{w}}{\pi g A_{0}^{3}} F_{0}=0, \omega_{j}=0
$$

where $A_{0}=\mathrm{w} h_{0}$ is the tidally averaged cross section area, and $F_{0}$ is the mean component of $\frac{1}{\pi} F=|Q| Q$, the signed square of the friction term.

The oscillatory components $\left(\omega_{j}>0\right)$ are determined by the wave equation. We obtain the wave equation by first differentiating the continuity equation in time and the momentum equation in space, and then eliminating the surface elevation $z$ by combining the equations:

$$
-\frac{1}{g h_{0} \mathrm{w}} \frac{\partial^{2} Q}{\partial t^{2}}+\frac{1}{\mathrm{w}} \frac{\partial^{2} Q}{\partial x^{2}}+\frac{1}{\mathrm{w}^{2}} \frac{\partial \mathrm{w}}{\partial x} \frac{\partial Q}{\partial x}-\frac{c_{d}}{g h_{0}^{3} \mathrm{w}^{2}} \frac{\partial Q|Q|}{\partial t}=0 .
$$

We approximate the signed square of the friction term with a quadratic Chebyshev polynomial (Dronkers, 1964):

$$
\begin{aligned}
\frac{1}{\pi} F & \approx|Q| Q, \\
F & =f_{0} Q_{h r}^{2}+f_{1} Q_{h r} Q+f_{2} Q^{2},
\end{aligned}
$$

where $Q_{h r}$ is half the tidal range. The complex conjugate is indicated by the asterisk. $f_{0,1,2}$ are coefficients that depend on the relative strength of river 
and tidal flow. $f_{0}$ is always small. When the river flow is low so that $Q_{0}<Q_{h r}$, then $f_{1}=\frac{8}{2}$ and $f_{2}$ is small. When the river flow is strong so that $Q_{0} \geq Q_{h r}$, then $f_{1}=0$ and $f_{2}$ equals $\pi$. Appendix 4.9 gives the detailed expressions for $f_{0,1,2}$.

The expansion of the discharge as a Fourier series (equation $4.1 \mathrm{~b}$ ), yields one equation for each frequency component. By continuity, the tidal discharge is proportional to its derivative with respect to time so that the wave equation reduces to a second-order ordinary differential equation (Ippen, 1966). As the surface elevation has been eliminated, the system consists only of one equation per frequency component:

$$
\frac{\partial^{2} Q_{j}}{\partial t^{2}}+g \frac{A_{0}}{\mathrm{w}^{2}} \frac{\partial \mathrm{w}}{\partial x} \frac{\partial Q_{j}}{\partial x}-g \frac{A_{0}}{\mathrm{w}} \frac{\partial^{2} Q_{j}}{\partial x^{2}}+\frac{c_{d} \mathrm{w}}{\pi A_{0}^{2}} F_{j}^{\prime}=0, \omega_{j}>0,
$$

where $\frac{1}{\pi} F_{j}^{\prime}$ are the frequency components of $\frac{\partial}{\partial t}(|Q| Q)$.

With the Chebyshev approximation, the frequency components $F_{j}$ and $F_{j}^{\prime}$ for $\omega_{j}=0, \omega_{j}=\omega_{1}$ and $\omega_{j}=2 \omega_{1}$ are:

$$
\begin{aligned}
& F_{0}=f_{0} Q_{h r}^{2}+f_{1} Q_{0} Q_{h r}+f_{2}\left(Q_{0}\left|Q_{0}\right|+\frac{1}{2}\left(\left|Q_{1}\right|^{2}+\left|Q_{2}\right|^{2}\right)\right), \\
& F_{1}^{\prime}=\mathrm{i} \omega_{1}\left(\left(f_{1} Q_{h r}+2 f_{2} Q_{0}\right) Q_{1}+f_{2} Q_{2} Q_{1}^{*}\right), \\
& F_{2}^{\prime}=\mathrm{i} \omega_{2}\left(\left(f_{1} Q_{h r}+2 f_{2} Q_{0}\right) Q_{2}+\frac{1}{2} f_{2} Q_{1}^{2}\right),
\end{aligned}
$$

where $\omega_{1}$ is the angular frequency of the main tidal species entering the river.

Further analysis is limited to two frequency components, representing the main tidal species. For the main tidal species we use the short hand notation:

$$
c_{2} \frac{\partial^{2} Q_{j}}{\partial x^{2}}+c_{1} \frac{\partial Q_{j}}{\partial x}+c_{0} Q_{j}=0
$$

with

$$
\begin{aligned}
& \frac{c_{1}}{c_{2}}=-\frac{1}{\mathrm{w}} \frac{\partial \mathrm{w}}{\partial x}, \\
& \frac{c_{0}}{c_{2}}=\frac{\omega_{1}^{2}}{g h_{0}}-\frac{\mathrm{i} \omega_{1} c_{d}}{\pi \mathrm{w} g h_{0}^{3}}\left(f_{1} Q_{h r}+2 f_{2} Q_{0}\right),
\end{aligned}
$$

where we consider the case in which the magnitude of the overtide is small so that its feedback on the main tidal species through $f_{2} Q_{2} Q_{1}^{*}$ can be neglected. As the frequency components are trigonometric functions in time (c.f equation 
4.1b), they are proportional to their derivative $\frac{\partial z_{j}}{\partial t}=i \omega_{j} z_{j}$. The surface elevation amplitude of each component can thus be determined by differentiating the discharge along $x$.

Substitution of the tidally average of the friction term (equation 4.8a) into the backwater equation (4.3) yields near the sea $h_{0} \approx z_{b}+$ $\left(f_{1} Q_{0} Q_{h r}+f_{2} Q_{0}\left|Q_{0}\right|\right) x$, which shows that the water surface slope increases linearly with the river discharge when the river discharge is low, and quadratically when it is high. Conversely, the frequency component of the friction term that corresponds to the main tidal species (equation 4.8b) increases linearly with the tidal discharge when the tidal discharge is low, and quadratically when it is high.

As the friction term is non-linear, it couples the equations between the frequency components. The friction term damps and delays the tide. In addition, it generates components of higher frequency, the overtide (Parker, 1991). The overtide changes the shape of the tidal wave as it propagates upriver (Parker, 1991). River flow forces an overtide with twice the frequency of the incoming tide so that high-water is advanced and low-water is delayed (Godin, 1999). This is the opposite to estuaries with wide tidal flats, where the falling limb of the tide is shorter in duration than the rising limb (Friedrichs and Madsen, 1992).

Similarly, the friction term generates lower frequency components, when the incoming tide contains components with close frequencies (LeBlond, 1979; Buschman et al., 2009). These modulate the daily mean water level over the spring neap-cycle. Subtidal variations of the surface elevation are captured by the $Q_{h r}$-terms in equation (4.8a). Modelling of subtidal harmonics is discussed in Kukulka and Jay (2003a). The overtide and subtidal harmonics are small in magnitude so that we ignore their feedback on the main tidal component in further analysis.

\subsubsection{Propagation of tidal waves}

The discharge and tidal amplitude can be expressed as the product of the initial values $Q_{j}(0), z_{j}(0)$ at the river mouth and a complex admittance factor that we define as:

$$
\begin{aligned}
& z_{j}(x)=z_{j}(0) \exp \left(-\mathrm{i} \int_{0}^{x} k_{j z} \mathrm{~d} x^{\prime}\right) \\
& Q_{j}(x)=Q_{j}(0) \exp \left(-\mathrm{i} \int_{0}^{x} k_{j Q} \mathrm{~d} x^{\prime}\right) .
\end{aligned}
$$


The along-channel change of the tidal wave $\left\{z_{1}, Q_{1}\right\}$ is thus uniquely determined by the wavenumbers $k_{1 Q}$ and $k_{1 z}$ as

$$
\begin{aligned}
\frac{1}{Q_{1}} \frac{\partial Q_{1}}{\partial x} & =-\mathrm{i} k_{1 Q}, \\
\frac{1}{z_{1}} \frac{\partial z_{1}}{\partial x} & =-\mathrm{i} k_{1 z}=\left(\frac{1}{k_{1 Q}} \frac{\partial k_{1 Q}}{\partial x}-\frac{1}{\mathrm{w}} \frac{\partial \mathrm{w}}{\partial x}-\mathrm{i} k_{1 Q}\right) .
\end{aligned}
$$

When the coefficients of equation (4.9a) are constant, then the wavenumbers are identical $k_{1 Q}=k_{1 z}=k_{1}$ and remain constant along the channel. This is only the case in a channel of constant width during normal river flow, i.e. when the tidally averaged depth does not change along the river. In this special case the frequency components of (4.1a) and (4.1b) become

$$
\begin{aligned}
z_{j}(t, x) & =z_{j}(t, 0) \exp \left(\mathrm{i}\left(\omega_{1} t-k_{1} x\right)\right), \\
Q_{j}(t, x) & =Q_{j}(t, 0) \exp \left(\mathrm{i}\left(\omega_{1} t-k_{1} x\right)\right) .
\end{aligned}
$$

The identity $\operatorname{Re}\left\{\exp \left(i\left(\omega_{1} t-k_{1} x\right)\right)\right\}=\exp \left(\operatorname{Im}\left\{k_{1}\right\} x\right) \cos \left(\omega_{1} t-\operatorname{Re}\left\{k_{1}\right\} x\right)$ reveals the two principal changes the tide undergoes while propagating upriver. First, while the tide travels upstream the wave is delayed in time at a rate equal to $\operatorname{Re}\left\{k_{1}\right\}$. In upstream parts, high water occurs later than downstream. Second, as friction dissipates energy, the tide is damped at a rate equal to $\operatorname{Im}\left(k_{1}\right)$. The tidal range is decreased in the upstream direction. The dimension of $k_{1}$ is one over length and assumes typical values in the order of $1 / 100 \mathrm{~km}^{-1}$. When the cross section geometry varies along the river, $k_{1 Q}$ and $k_{1 z}$ vary as well. The remainder of this section shows how $k_{1 Q}$ depends on the river discharge and on variation in cross section geometry.

The wave equation (4.9a) can be separated into two first-order ordinary differential equations when equation (4.11a) is inserted into equation (4.9a). This yields a Riccati equation, from which $k_{1 Q}$ can be obtained:

$$
\frac{\partial k_{1 Q}}{\partial x}=\mathrm{i} k_{1 Q}^{2}-\frac{c_{1}}{c_{2}} k_{1 Q}-\mathrm{i} \frac{c_{0}}{c_{2}} .
$$

Far upstream, the flow is uniform and $k_{1 Q}$ does not change along the channel. The left-hand side of equation (4.13a) is then zero so that $-i k_{1 Q}$ is a root of the characteristic polynomial $c_{2} r^{2}+c_{1} r+c_{0}=0$. The roots of the characteristic polynomial are

$$
r^{ \pm}(x)=\frac{\mathrm{i}}{2 \mathrm{w}} \frac{\partial \mathrm{w}}{\partial x} \pm \sqrt{\frac{\omega^{2}}{g h}-\frac{1}{4 \mathrm{w}^{2}}\left(\frac{\partial \mathrm{w}}{\partial x}\right)^{2}+\frac{\mathrm{i} c_{d} \omega}{\pi \mathrm{w} g h^{3}}\left(2 f_{2} Q_{0}+f_{1} Q_{1}\right)} .
$$


For a river of constant width and depth, the roots remain constant along the channel. In this case, the roots have well-known limits for the case of no river flow: $r^{2}=-\frac{\omega^{2}}{g h}+\mathrm{i} \frac{8}{3 \pi} c_{d} \omega \frac{Q_{h r}}{g \mathrm{w} h^{3}}$, (Lorentz, 1926) and when the river flow is strong: $r^{2}=2 \mathrm{i} \omega c_{d} \frac{Q_{0}}{g \mathrm{w} h_{0}^{3}}$ (Godin, 1985). When the width changes along the channel, the roots can still remain constant as long as the change is exponential.

Downstream, where both width and depth converge, $k_{1 Q}$ changes along the river. Thus $k_{1 Q}$ can be determined by integrating the initial value problem (4.13a) from upstream to downstream. In general, there is no closed form solution to this initial value problem. Further simplifications are necessary to determine how the tide propagates upriver.

\subsubsection{Wave propagation along rivers with a gradually varying cross section}

The solution to the wave equation is the superposition of two waves. One wave travels upstream and the other one travels downstream. These are analogous to the Riemann invariants of the shallow water equations. In the case of constant coefficients, which holds in channels of constant cross section:

$$
\begin{aligned}
Q_{1}(x) & =Q_{1}^{+}(0) \exp \left(r^{+} x\right)+Q_{1}^{-}(0) \exp \left(r^{-} x\right), \\
z_{1}(x) & =z_{1}^{+}(0) \exp \left(r^{+} x\right)+z_{1}^{-}(0) \exp \left(r^{-} x\right),
\end{aligned}
$$

where $r^{ \pm}$are the two roots of the characteristic polynomial. The signs of the real and imaginary parts of the roots are equal. The positive root corresponds to the seaward travelling wave and the negative root to the landward travelling wave. The real part of the wavenumber $k_{1}$ is positive and its imaginary part is negative, as $k_{1}=+\mathrm{i} r^{-}$. When no wave enters at the upstream end, then $Q_{1}^{+}(x)=0, z_{1}^{+}(x)=0$ and $Q_{1}=Q_{1}^{-}, z_{1}=z_{1}^{-}$.

The wave propagates as a pure exponentially damped sine. The rate at which it travels corresponds to the imaginary part and at which it is damped to the real part of the root, respectively. When the cross section geometry varies along the channel, then the incoming wave is partially reflected. It follows from equation (4.13a) that in this case, the wavenumber differs from the roots of the characteristic polynomial. The coefficients $c_{\{0,1,2\}}$ vary as well 
and the wave propagates as

$$
\begin{gathered}
\frac{\partial Q_{1}^{-}}{\partial x}=(r^{-}+\underbrace{\frac{-1}{r^{-}-r^{+}} \frac{\partial r^{-}}{\partial x}}_{T^{-}}) Q_{1}^{-}+\underbrace{\frac{-1}{r^{-}-r^{+}} \frac{\partial r^{+}}{\partial x}}_{R^{+}} Q_{1}^{+}, \\
\frac{\partial Q_{1}^{+}}{\partial x}=\underbrace{\frac{+1}{r^{-}-r^{+}} \frac{\partial r^{-}}{\partial x}}_{R^{-}} Q_{1}^{-}+(r^{+}+\underbrace{\frac{+1}{r^{-}-r^{+}} \frac{\partial r^{+}}{\partial x}}_{T^{+}}) Q_{1}^{+},
\end{gathered}
$$

which is derived in Appendix 4.10. $T^{-}$and $T^{+}$are the coefficients of transmission, whereas $R^{-}$and $R^{+}$are the coefficients of reflection of the upstream and downstream travelling waves, respectively.

When the cross section geometry varies smoothly at a low rate, then the amplitude of the reflected wave is negligible so that $Q_{1} \approx Q_{1}^{-}$and $k_{1} \approx$ $-\mathrm{i}\left(r^{-}+\frac{1}{r^{-}-r^{+}} \frac{\partial r^{-}}{\partial x}\right)$. Thus even when the reflected wave is small, the incoming wave can change considerably by transmission. For infinitesimally small waves $r^{ \pm}$does not depend on $Q_{1}$ and (4.15a) gives direct insight into the propagation of the tidal wave along a river with known geometry.

For the sake of illustration, consider the case where the width remains constant along the channel so that $r^{-}=-r^{+}$. When the cross section geometry changes smoothly at a low rate, equation (4.15a) and (4.15b) simplify to

$$
\begin{gathered}
\frac{1}{Q_{1}} \frac{\partial Q_{1}}{\partial x}=r^{-}-\frac{1}{2 r^{-}} \frac{\partial r^{-}}{\partial x}, \\
\frac{1}{z_{1}} \frac{\partial z_{1}}{\partial x}=r^{-}+\frac{1}{2 r^{-}} \frac{\partial r^{-}}{\partial x},
\end{gathered}
$$

where higher powers of $\frac{\partial r^{ \pm}}{\partial x}$, as well as higher derivatives are neglected, as only a small part of the wave is reflected when the geometry changes gradually.

Equations (4.15b) and (4.15a) show that a convergence of the cross section has the opposite effect on the upstream travelling wave $\left(Q^{-}, z^{-}\right)$and reflected waves $\left(Q^{+}, z^{+}\right)$, as the sign in front of $\frac{\partial r^{ \pm}}{\partial x}$ is equal. In contrast, friction damps the incoming and outgoing waves at the same rate, as the sign in front $r^{ \pm}$is the opposite. For the same reason, equations (4.16a) and (4.16b) show that convergence has likewise the opposite effect on the discharge $Q_{1}$ and surface elevation $z_{1}$, while they are also damped at the same rate.

\subsubsection{Decomposition into recursively reflected waves}

The propagation of the tide up-river can be approximated by a single integral (equation 4.10a) and an approximation of the wave number (equation 4.16a). 
However, this neglects the reflected wave. When the cross section geometry or friction changes over a distance that is short compared to the wave length, then a part of the wave is reflected (Lighthill, 2001). Reflection of tidal waves in rivers and estuaries is therefore not entirely negligible, as the wave length extends over hundreds of kilometres. As the reflected wave travels along the same channel, it is likewise partially reflected, so that recursive transmission and reflection occurs (Witting, 1981). As small waves pass through each other without interacting, we can decompose the incoming and outgoing wave into a series that we interpret as simultaneously recursively reflected waves:

$$
Q_{1}=\sum_{n=0,1, \ldots} Q_{1}^{(n)}=\sum_{n=0,2, \ldots}\left(Q_{1}^{(2 n),-}+Q_{1}^{(2 n+1),+}\right) .
$$

A wave that enters from the left develops as

$$
Q_{1}^{(0),-}(x)=Q_{1}^{-}(0) \exp \left(\int_{0}^{x} T^{-} \mathrm{d} x^{\prime}\right) .
$$

The subsequent terms of the series are given by double-integrals (Wilmer III and Costa, 2008). Each upstream travelling wave generates another downstream travelling wave, and each downstream travelling wave generates another upstream travelling wave:

$$
\begin{aligned}
Q_{1}^{(2 n+1),+} & =\int_{x}^{\infty} R^{-} Q_{1}^{(2 n),-} \exp \left(\int_{x}^{x^{\prime}} T^{+} \mathrm{d} x^{\prime \prime}\right) \mathrm{d} x^{\prime}, \\
Q_{1}^{(2 n),-} & =\int_{0}^{x} R^{+} Q_{1}^{(2 n-1),+} \exp \left(\int_{x^{\prime}}^{x} T^{-} \mathrm{d} x^{\prime \prime}\right) \mathrm{d} x^{\prime}, n>0 .
\end{aligned}
$$

As only a small part of the wave is reflected and its amplitude is attenuated, the integrals remain finite. The series (4.17) converges rapidly when the channel geometry varies smoothly or when the damping is strong. In this case, the magnitude of the reflected wave is small so that the first term of the series (4.18) approximates the admittance of the tide well. A comparison between the exact solution (4.10b) and its approximation (4.18) reveals that the wavenumber corresponds to to the sum of the root of the characteristic polynomial and the coefficient of transmission $k_{1 Q} \approx-\mathrm{i}\left(r^{-}+T^{-}\right)$.

A series expansion of the wavenumber reveals how the tide propagates depending on the cross section geometry. Gravity and friction always act on the tide, even if the cross section geometry does not vary along the river (equation 4.2b). They form the zero-order terms. Convergence only occurs when the cross section geometry varies along the river and is hence represented by the partial derivatives. The admittance is consequently the product of two factors. The first accounts for the effect of gravity and friction, and the second for the effect of width and depth convergence. 


\subsubsection{The effect of gravity and friction}

When only gravity acts on the wave, i.e. when both friction and $\frac{\partial \mathrm{w}}{\partial x}$ are zero, and the wavenumber (equation 4.16a) is identical to i-times the root of the characteristic polynomial (equation $4.13 \mathrm{~b}$ ), and simplifies to

$$
k_{1,0}=\frac{\omega_{1}}{\sqrt{g h}}, a=0 .
$$

When both gravity and friction act, i.e. $\frac{\partial \mathrm{w}}{\partial x}$ is zero, the wavenumber is:

$$
k_{1, a}=k_{1,0} \sqrt{1+2 \mathrm{i} a}
$$

where $a$ is measures the strength of friction (c.f. equation $4.8 \mathrm{~b}$ ):

$$
a=\frac{1}{\pi} \frac{c_{d}}{\omega \mathrm{w} h_{0}^{2}}\left(f_{2} Q_{0}+\frac{1}{2} f_{1} Q_{h r}\right) .
$$

The wave travels into the direction in which it is driven by gravity and friction acts against it. The surface amplitude and discharge are thus affected in the same manner, $k_{1,0 Q}=k_{1,0 z}$. The friction scale $a$ varies along the river and with the strength of the flow. A Puiseux series expansion with respect to the parameter $a$ reveals the effect of friction for low river flow

$$
k_{1, a}=k_{1,0}(1+\mathrm{i} a), a \rightarrow 0
$$

and high river flow, respectively:

$$
k_{1, a}=k_{1,0}(1+\mathrm{i}) \sqrt{a}, a \rightarrow \infty
$$

The rates of damping and phase change are as before determined by the imaginary and real parts of equation (4.23a) and equation (4.23b), respectively. Substitution of equation (4.20) and equation (4.22) reveals the effect of depth convergence: when friction is low, the tide is damped proportionally to the discharge and $h_{0}^{-5 / 2}$. The phase changes at a rate that is independent of the friction. When the friction is high, then the rate of damping and phase change are both proportional the square root of the discharge and $h_{0}^{-3 / 2}$.

\section{Low river flow}

When the tidal discharge is much larger than the river discharge, then the friction coefficients in equations (4.8a) and (4.8b) attain the values $f_{1}=3 / 8$ and $f_{2}=0$. This is identical to the approximation by Lorentz (Terra et al., 2005). In this case,

$$
a=\frac{c_{d}}{\omega \mathrm{w} h_{0}^{2}} \frac{8}{3 \pi} Q_{1}\left(1-4 \frac{Q_{0}^{2}}{\left|Q_{1}\right|^{2}}\right),\left|Q_{1}\right| \gg\left|Q_{0}\right| .
$$



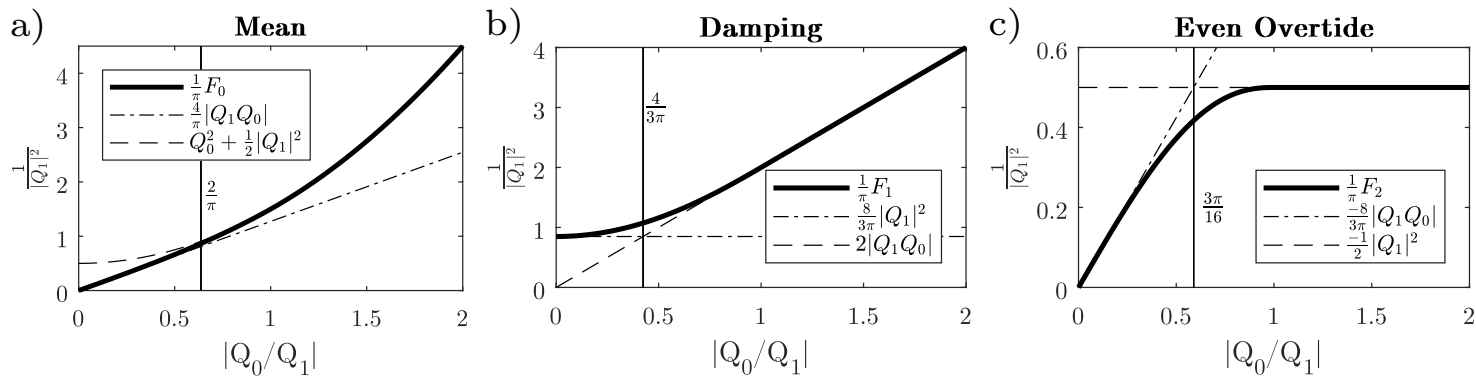

Figure 4.5: Magnitudes of the frequency components of the friction term (equations (4.8a)-(4.8c), bold), depending on the relative strength of river and tidal flow $\left(\left|Q_{0}\right| /\left|Q_{1}\right|\right)$, as well as their low flow asymptotes $\left(\left|Q_{0}\right| /\left|Q_{1}\right| \rightarrow 0\right.$, dashdotted) and high flow asymptotes $\left(\left|Q_{0}\right| /\left|Q_{1}\right| \rightarrow \infty\right.$, dashed). Note that the discharge scale is identical to the velocity scale as $\left|u_{0}\right| /\left|u_{1}\right|=\left|Q_{0}\right| /\left|Q_{1}\right|$.

Damping is thus asymptotically insensitive to river discharge when the tide is strong. River discharge does not add noticeably to the damping as long as $Q_{0}<\frac{4}{3 \pi}\left|Q_{1}\right|$ (figure 4.5b). The water depth increases linearly with the river discharge when the river discharge is low, as from equations (4.3) and (4.8a) it follows that $\left.h_{0} \approx h_{0}\right|_{Q_{0}=0}+x \frac{8}{3 \pi} \frac{c_{d} w}{g A_{0}^{3}} Q_{h r} Q_{0}$ (figure 4.5a). Damping can thus even decrease with the river discharge before a threshold is reached, as the linear increase in water depth can reduce the friction by a larger amount than it is raised by the square of the river discharge, as long as the river flow does not considerably increase the roughness.

\section{Strong river flow}

When the river discharge is so strong that the flow does not reverse over the tidal cycle, then the friction coefficients (4.8a) and (4.8b) obtain the values $f_{1}=0$ and $f_{2}=\pi$.

$$
a=\frac{c_{d}}{\omega \mathrm{w} h_{0}^{2}} 2 Q_{0},\left|Q_{0}\right|>\left|Q_{1}\right| .
$$

The tide only contributes to the damping rate by modulating the water depth, when the river discharge is large, which does not affect the first order approximation of the damping. 


\subsubsection{The Effect of width and depth convergence}

Width and depth convergence modify the wavenumber by the term $\Delta k_{1}$. When the cross section geometry changes smoothly along the river, the term is:

$$
\begin{aligned}
\Delta k_{1} & =\frac{1}{4(\mathrm{i}-a)}\left((1+3 \mathrm{i} a) \frac{1}{h_{0}} \frac{\partial h_{0}}{\partial x}+(2+3 \mathrm{i} a) \frac{1}{\mathrm{w}} \frac{\partial \mathrm{w}}{\partial x}\right), \\
k_{1 z} & =k_{1, a}+\Delta k_{1}, \\
k_{1 Q} & =k_{1, a}-\Delta k_{1} .
\end{aligned}
$$

This is obtained by substituting the roots of the characteristic polynomials (equation (4.13b)) in equation (4.15a), followed by omitting higher order derivatives as well as higher powers of the first derivatives.

In contrast to damping, width and depth convergence have the opposite effect on the surface amplitude and the discharge. This corollary of Green's law (Jay, 1991; Green, 1838) thus also holds in the presence of friction. The sign of the convergence term also depends on the direction in which the wave travels and in which the cross section changes. Convergence raises the tidal amplitude when the cross section becomes narrower and shallower.

In the limit of low friction, the change of wavenumber with the rate of convergence is

$$
\Delta k_{1}=-\mathrm{i} \frac{1}{4} \frac{1}{h_{0}} \frac{\partial h_{0}}{\partial x}-\mathrm{i} \frac{1}{2} \frac{1}{\mathrm{w}} \frac{\partial \mathrm{w}}{\partial x}, a \rightarrow 0 .
$$

A relative change of width thus has a larger effect than a relative change in depth of the same magnitude. This is known as Green's Law (Jay, 1991). When friction is strong, the rate of convergence is

$$
\Delta k_{1}=-\mathrm{i} \frac{3}{4} \frac{1}{h_{0}} \frac{\partial h_{0}}{\partial x}-\mathrm{i} \frac{3}{4} \frac{1}{\mathrm{w}} \frac{\partial \mathrm{w}}{\partial x}, a \rightarrow \infty .
$$

Strong friction enhances the effect of convergence, and in contrast to low friction, there, relative changes of width and depth have the same effect.

However, the river discharge influences the effect of convergence not only indirectly by increasing friction, but also directly, as depth convergence decreases with increasing discharge as $\frac{\partial h_{0}}{\partial x}=\frac{\partial z_{0}}{\partial x}-\frac{\partial z_{b}}{\partial x}$ (figure 4.6a). The effect of width and depth convergence thus increases monotonously with increasing tidal discharge (figure 4.6b), but the effect of depth convergence increases with the river discharge only when the river discharge is low, and approaches zero for normal flow. Both width and depth convergence primarily affect the amplitude. The rate of phase change is only affected when friction is intermediate. 

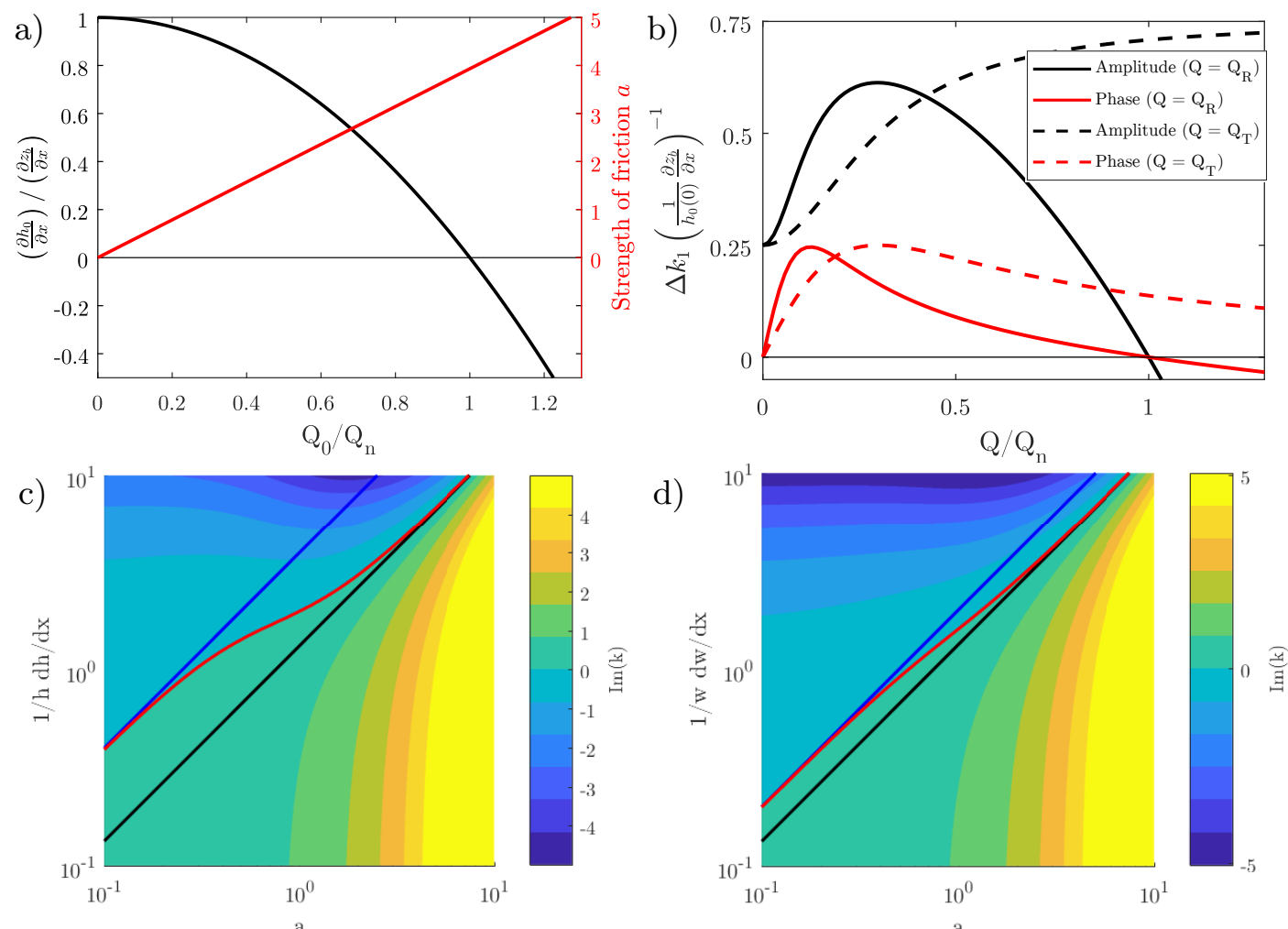

Figure 4.6: a) Relative depth convergence (black) and friction scale (red) at the river mouth depending on the river discharge for infinitesimal waves; b) Effect of depth convergence on the damping rate (black) and rate of phase change (red) depending on the river discharge (solid) or tidal discharge (dashed) c) Damping rate depending on friction and depth convergence as approximated by equation (4.26a), red line shows critical convergence $(\operatorname{Im}(k)=0)$, blue and black are asymptotes for high and low friction, respectively

\subsection{Hydrodynamics of tidal rivers with a sloping bed}

This section considers a river with moderate bed slope, where reflection along the channel is small. For illustration, it adopts dimensions that are similar to those of the Kapuas river. At the downstream boundary, the amplitude of the incoming wave is prescribed, and the reflected wave is allowed to pass freely, i.e. without reflection, to the sea. If not otherwise mentioned, the amplitude of this wave is infinitesimal so that the damping is entirely caused by the river flow (equation 4.25). The computational domain ends upstream of the point where the bed reaches sea level, where the tidal wave is allowed to leave the domain without reflection. The examples contrast the propagation of the tide in the presence of backwater effects to that predicted with a conventional 
model that assumes the tidally averaged water depth to remain constant along the channel and not to change with the river discharge.

\subsubsection{Tidally averaged water level}

The tidally averaged water level changes along a river depending on the river discharge. When the river discharge is low, it forms a backwater profile, and depth decreases in the upstream direction (figure 4.8 black and red). Far upstream, the water surface slope asymptotically approaches the bed slope, so that the tidally averaged water depth remains constant along the river.

There is no analytic solution to the backwater profile (eq. (4.3)), beside those of the Bresse type (Vatankhah and Easa, 2011), which cannot be integrated into a general solution of the tide, as they swap the dependent and independent variables. For the analysis of the river tide, we thus linearize the backwater equation (equations (4.3) and (4.8a)) at the river mouth,

$$
h_{0}(x)=h_{0}(0)+\left(\left.\frac{\partial z_{0}}{\partial x}\right|_{0}-\frac{\partial z_{b}}{\partial x}\right) x+O\left(\left.\frac{\partial^{2} z_{0}}{\partial x^{2}}\right|_{0} x^{2}\right)=\frac{c_{d}}{g} \frac{Q_{0}\left|Q_{0}\right|}{\mathrm{w}^{2} h_{0}^{3}},
$$

for the reach between the river mouth and the point where the flow becomes approximately uniform $\left(x=\left|h_{0}(0)-h_{u}\right|\left(\left.\frac{\partial h_{0}}{\partial x}\right|_{0}\right)^{-1}\right) . h_{u}$ is the depth in the reach of uniform flow far upstream.

When the river is at normal flow $\left(Q=Q_{n}\right)$, then the water surface slope is identical to the bed slope and the tidally averaged water depth does not change along the river (blue in figure 4.7a). When the river discharge is below normal flow, then the river forms a backwater curve (black, red and green in figure 4.7a). When the river discharge is above normal flow, then the water surface forms a drawdown curve so that the depth increases into the upstream direction (orange in figure 4.7a). For extremely low river discharge, the point where the flow becomes uniform approaches the point where the bed reaches sea level $x=L_{0}=h_{0}\left(\frac{\partial z_{b}}{\partial x}\right)^{-1}$. As long as the river is in a state of backwater, this point is located closer to the river mouth, the higher the discharge (figure 4.7b). As the wavenumber depends on the water depth, the backwater profile strongly influences the admittance of the tide.

\subsubsection{Admittance along the river}

In the case of normal flow, the depth does not converge. The tidal amplitude decreases exponentially along the channel so that the tidal amplitude drops most rapidly near the sea and less rapidly farther upstream (figure 4.8b). The 


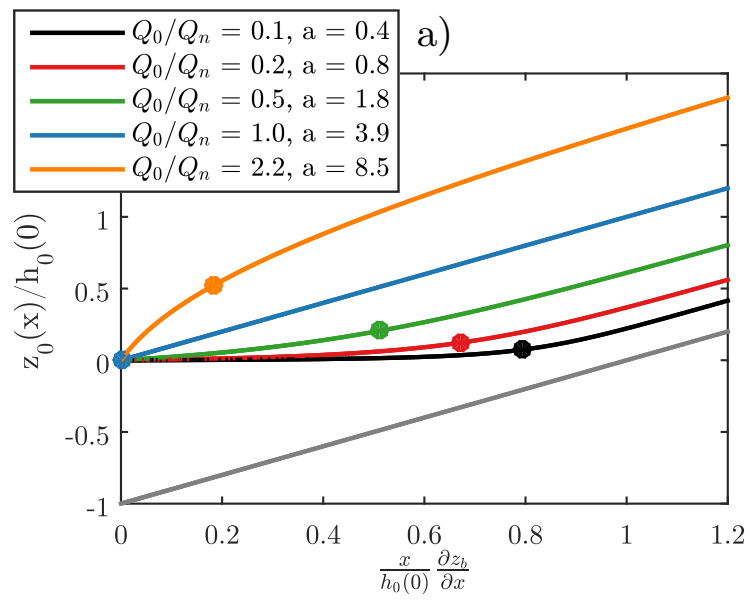

b)

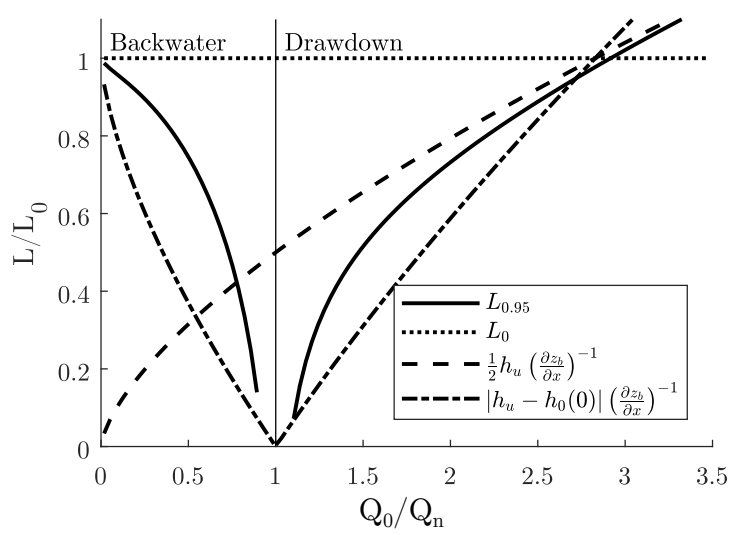

Figure 4.7: a) Tidally averaged water level along river, the backwater drawdown lengths are indicated by dots; b) Backwater and drawdown length $L$ for various states of river flow, defined as distance to point where water depth deviates no more than $5 \%\left(L_{95}\right)$ and approximated by simplified relations
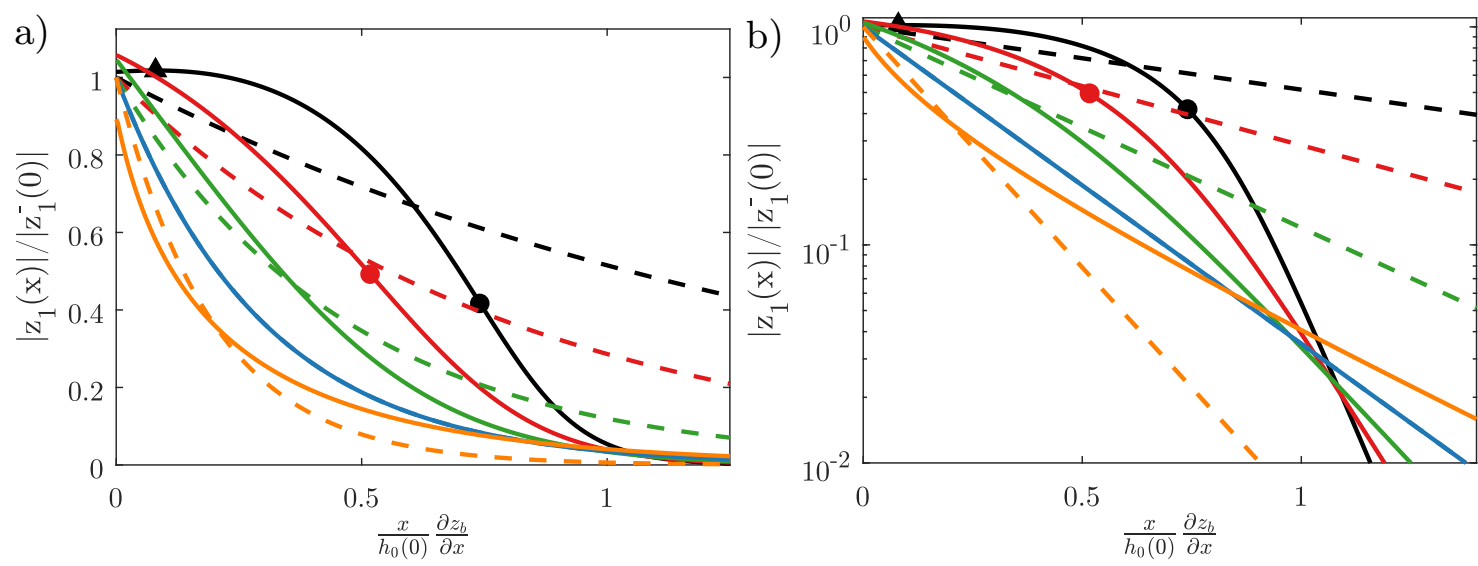

Figure 4.8: a) Admittance of the tide for the case that the depth varies along the river (bold) and for the case that the depth remains constant along the river (dashed); inflexion points are indicated by dots, maxima by chevrons; b) the logarithmic y-axis reveals the inversion of stage-amplitude relation in the asymptotic reach; scenarios as in figure $4.7 \mathrm{a}$

wave propagates with constant celerity (figure 4.9a). At normal flow, the frictional damping is strong so that the damping rate is proportional to the square root of the river discharge as given by equation (4.23a). This is a well-known relation for the propagation of a tidal wave along a river with constant depth (Jay and Flinchem, 1997; Godin, 1985; LeBlond, 1978; Jay, 1991).

However, when the river is not at normal flow, then the depth changes along 
the river and the tide propagates differently to the case where the depth remains constant along the river. At low flow (black and red lines in 4.8a), the tidally averaged water surface forms a backwater profile. The tidal amplitude decreases only slowly near the river mouth, and more rapidly farther upstream (black and red in 4.8b). The transition is marked by an inflexion point at which $\frac{\partial^{2}\left|z_{1}\right|}{\partial x^{2}}=0$. As equation (4.11b) implies $\frac{1}{\left|z_{1}\right|} \frac{\partial\left|z_{1}\right|}{\partial x}=\operatorname{Im}\left(k_{1 z}\right)$, this implies

$$
\frac{\partial \operatorname{Im}\left(k_{1 z}\right)}{\partial x}-\operatorname{Im}\left(k_{1 z}\right)^{2}=0 .
$$

The inflexion point is located closer to the sea for higher river discharges, similar to the transition point where the asymptotic flow is reached. For discharges higher than a particular value, the inflexion point is not observed anymore. Only when the river discharge is above this threshold, the tide is admitted similarly to the conventional case where the depth is assumed to be constant along the river.

For very low discharges (black), the tidal range even increases in landward direction, to a point where a maximum is reached. The tidal admittance is hypersynchronous. At this point, the imaginary part of the wave number is zero,

$$
\operatorname{Im}\left(k_{1 z}\right)=0 .
$$

The maximum is located closer to the sea than the inflexion point. This point is related to the concept of critical damping that is used to characterize tidally dominated estuaries (Jay, 1991). Along such estuaries, width convergence can cancel frictional damping. If the tidally averaged water depth remains constant along an estuary, it can be critically damped along its entire length so that the tidal amplitude neither increases nor decreases, in which case an estuary is considered to be "ideal". A fundamental difference between tidedominated estuaries and tidal rivers with a sloping bed is thus that tidal rivers cannot represent ideal estuaries, as the damping can be critical only at one point, and not along the entire tidally influenced reach. The location of this point furthermore depends on the river discharge. Similar to the location of the inflexion point, the maximum is located closer to the sea for higher river discharges and vanishes when the discharge exceeds a certain threshold.

\subsubsection{Damping and convergence rates}

The rate of amplitude change along the river is the combined effect of frictional damping and convergence of the width and depth. For large depths, 

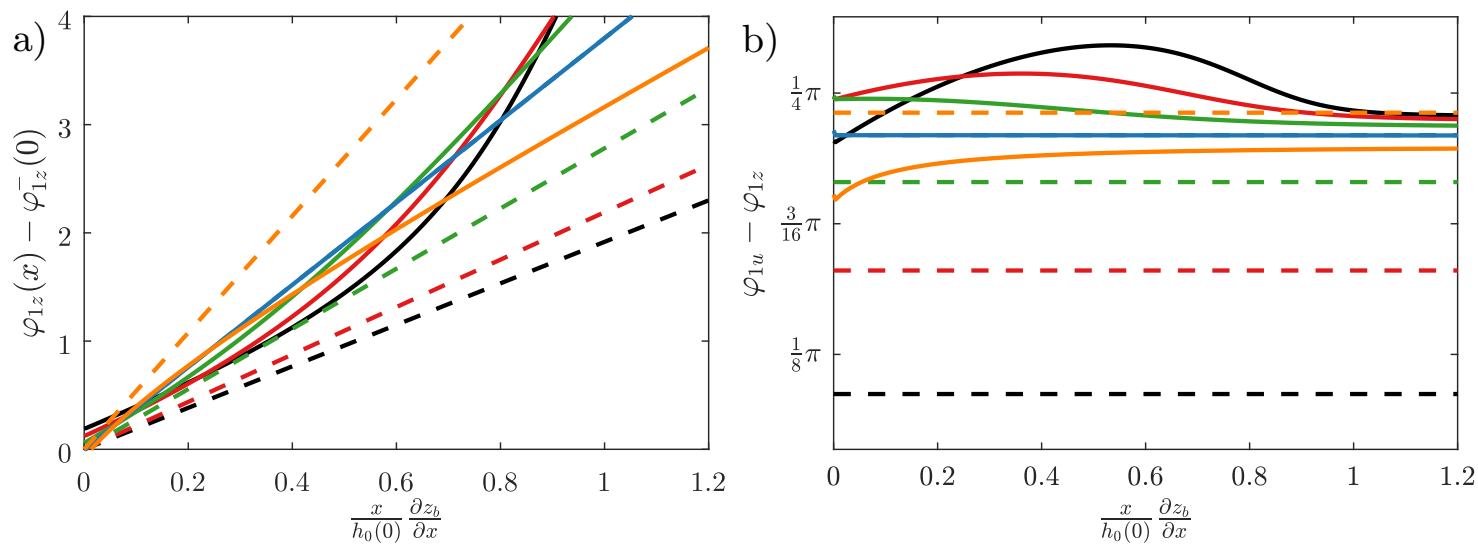

Figure 4.9: a) Phase difference between the tidal surface elevation along the river with respect to that at the mouth; b) phase lag between the tidal velocity and surface level variation along the river; without backwater effects, the wave is near progressive during low flow (black dashed), and between progressive and standing during high flow (orange dashed), with backwater effect the waves are always between progressive and standing (solid lines); scenarios as in figure 4.8

the damping rate is proportional to $h_{0}^{-5 / 2}$ (equation 4.23a), while depth convergence is proportional to $h_{0}^{-1}$ (equation 4.26a). At low flow, the river forms a backwater profile and the depth increases towards the sea. The effects of both the frictional damping and convergence thus decrease towards the sea, but frictional damping decreases more rapidly so that close to the sea the effect of convergence is larger than that of damping, and the amplitude of waves travelling upstream increases along the downstream part of the tidal river. In this reach, the imaginary part of $k_{1 z}$ is thus positive (figure 4.10). Conversely, the water depth decreases into the upstream direction towards the point where the bed reaches sea level when the river discharge is low. For small depths, damping is proportional to $h_{0}^{-3 / 2}$ while convergence is still proportional to $h_{0}^{-1}$. Both the effects of damping and convergence thus increases into the upstream direction, but damping increases more rapidly so that eventually frictional damping dominates, and the tidal amplitude is reduced in the upstream part of the tidal river. In this reach, the imaginary part of $k_{1 z}$ is thus negative (figure 4.10). Only when the river discharge is very large so that it forms a drawdown curve, depth increase into the upstream direction. In that case, damping is very strong, and convergence has the opposite effect compared to the situation for low flow so that the tidal amplitude rapidly decreases. 

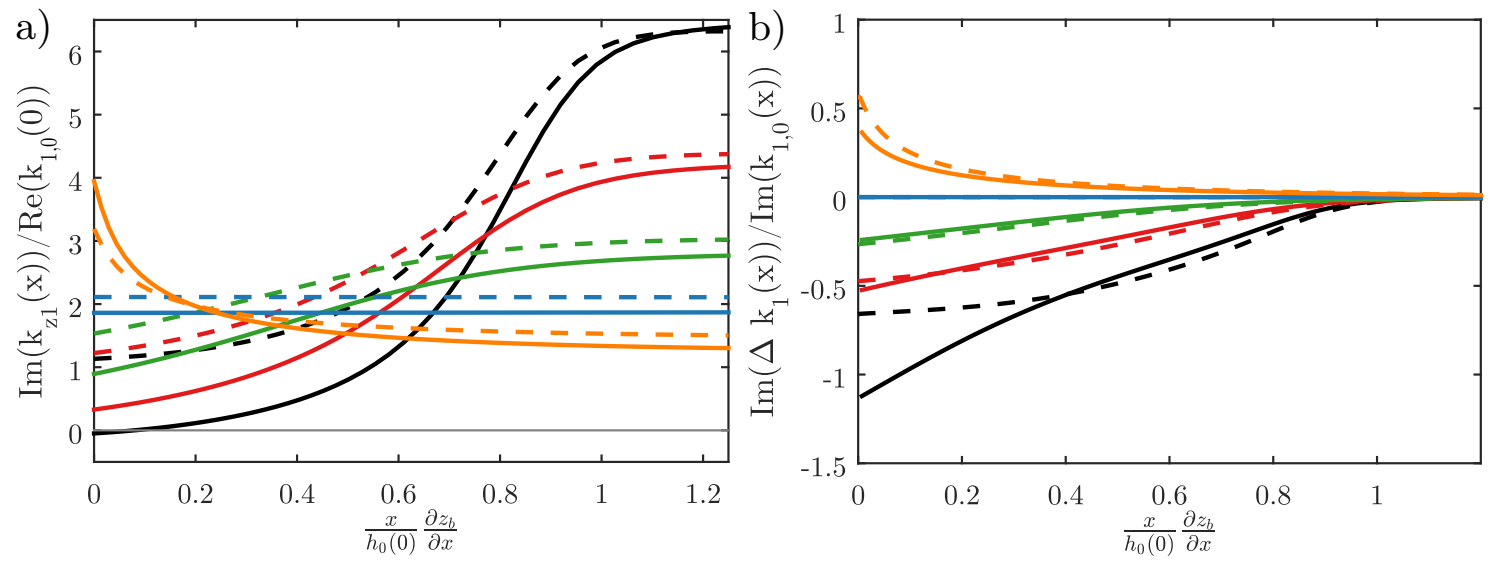

Figure 4.10: a) Imaginary (dashed) and real (solid) parts of the wavenumber $z_{1}$, corresponding to the rate of damping and phase change, respectively; critical damping occurs at $\operatorname{Im}\left(k_{1 z}\right)=0$. b) Relative strength of convergence compared to that of gravity and friction $\left(\operatorname{Im}\left(\Delta k_{1} / k_{1,0}\right)\right.$, solid) and its approximation by equation (4.26a) (dashed), critical damping occurs at -1 ; scenarios correspond to those in figure 4.8

\subsubsection{Asymptotic admittance}

The influence of the river discharge on the propagation of the tide in the upstream reach where the flow becomes uniform has been studied by Godin (1985). However, this study does not pay attention to the influence of the river flow on the tidally averaged water depth. When the slope of the riverbed is very small, then tidally averaged water depth does not strongly change with the river discharge. In such a case the damping decreases with the river discharge so that the tide intrudes far upstream during low flow (figure 4.8b). However, this is not the case when the slope of the riverbed is not small. The admittance is always marginal beyond the point where the bed reaches sea level (figure 4.8b). In the asymptotic reach (inset in figure 4.8), the tidal amplitude even slightly increases with the river discharge. This is a consequence of backwater dynamics. Far upstream, the river flow becomes asymptotically uniform. The ordinary differential equation for the water surface elevation (4.3) simplifies to an algebraic equation referred to as the Chézy formula. This allows one to eliminate either the river discharge or the water depth from the expression for the wavenumber. Far upstream, damping is strong so that the 
wavenumber is approximated by equation $(4.23 \mathrm{~b})$ :

$$
\begin{aligned}
k_{1 z} & =-(1-\mathrm{i}) \sqrt{\frac{c_{d} \omega Q_{0}}{g \mathrm{w} h_{0}^{3}}}, \\
& =-(1-\mathrm{i}) \sqrt{\frac{\omega \mathrm{w}}{Q_{0}} \frac{\partial z_{b}}{\partial x}}, \\
& =-(1-\mathrm{i}) \sqrt[4]{\frac{c_{d} \omega^{2}}{g h_{0}^{3}} \frac{\partial z_{b}}{\partial x}}, x>\left(\frac{1}{h_{0}} \frac{\partial z_{b}}{\partial x}\right)^{-1} .
\end{aligned}
$$

As intuition suggests, the damping is the stronger, the larger the slope, due to the higher flow velocity and shallower depth. The expression also shows that damping is indeed asymptotically inversely proportional to the square root of the river discharge so that in the asymptotic reach, the amplitude increases with the river discharge, which is the opposite to what is predicted by conventional models, where the depth remains constant along the river. The expression bears yet another surprise: neither the rate of frictional damping nor that of the phase change do asymptotically depend on the drag coefficient for a particular river discharge. This implies that tidal damping along the upstream reach has to be calibrated by adjusting other parameters, such as bed slope or channel geometry, rather than the drag coefficient, which is a common parameter for the calibration of hydrodynamic models. Similarly, the rate of damping and phase change are asymptotically independent of the water depth for a particular river discharge.

We point out that upstream propagation of tidal waves is not primarily inhibited by the pull of gravity down the slope, but rather by high friction caused by either the low water depth during low discharges, or by high flow velocities during high discharges. For small discharges, the region where the damping is low extends farther upstream, yet in the asymptotic reach beyond the point where the bed reaches sea level, the tidal amplitude always decreases. This implies that the transition between the reach where friction is low and the asymptotic reach is more rapid for higher discharges.

\subsubsection{Tidal propagation where the bed reaches sea level}

When the river discharge is very low, the water is shallow near the point where the bed reaches sea level so that the relative change of depth along the river is large. The wave is therefore partially reflected according to equation (4.15a). Locally, the reflected wave adds noticeably to the water surface oscillation, but as it travels back, it rapidly decreases in amplitude, because the friction 
is high and divergence of the cross section area reduces the amplitude of the reflected wave. Near the head of the tidal river, the amplitude of the main tidal species can exceed that of the water depth. The overtide rises low water by changing the shape of the tide so that the riverbed does not necessarily fall dry during periods of low river flow.

Without river discharge, the bed dries up beyond the point where the bed reaches sea level so that the tide cannot propagate much farther upstream. The model (4.9a) forces the tidal amplitude to zero at the point where the bed reaches sea level. The phase of the tide changes at an ever-increasing rate towards the point where the depth reaches zero so that the phase is undefined at this point. This is the case for all waves of zero amplitude and not an artefact of the model. In practice, a short reach upstream of the head of the tidal river can periodically flood and dry over the tidal cycle. The flow furthermore concentrates near the thalweg and shallow parts of the cross section are periodically flooded. A model that has a bed that is not flat across the river can better predict how the tide propagates near the head. However, the solution is sensitive to unevenness of the riverbed, to seasonal variation of the sea level as well as to the residual river flow. Wetting and drying of the entire cross section is also beyond the scope of the shallow water equations (4.2a) and ( $4.2 \mathrm{~b})$, as those require the depth to be non zero at all time. In any way, the tide does not propagate far beyond the point where the bed reaches sea level during periods without river flow, as it rapidly decays due to the shallow depth as well as storage on the higher parts of the bed which are periodically flooded. The case of the wave vanishing when running up the slope thus contrasts with the case where the wave runs against a wall, where the surface amplitude obtains a maximum, as well as the case with a wave propagation along a channel with a horizontal bed, where the wave propagates incessantly, without ever entirely decaying to zero. A minimum base flow assures that the bed of the Kapuas never falls entirely dry. However, bars and dunes emerge during low flow beyond the point where the bed reaches sea level, which is slightly upstream of the city of Sanggau.

\subsubsection{At-a-station admittance}

The tidal amplitude that is observed at a gauging station depends on the river discharge, in analogy to the admittance of the tide along the river (section 4.4.2). Figure 4.11 shows the admittance of the tide at three stations along a tidal river with a sloping bed in comparison to the admittance that is predicted by a conventional model, which neglects the backwater dynamics and assumes 

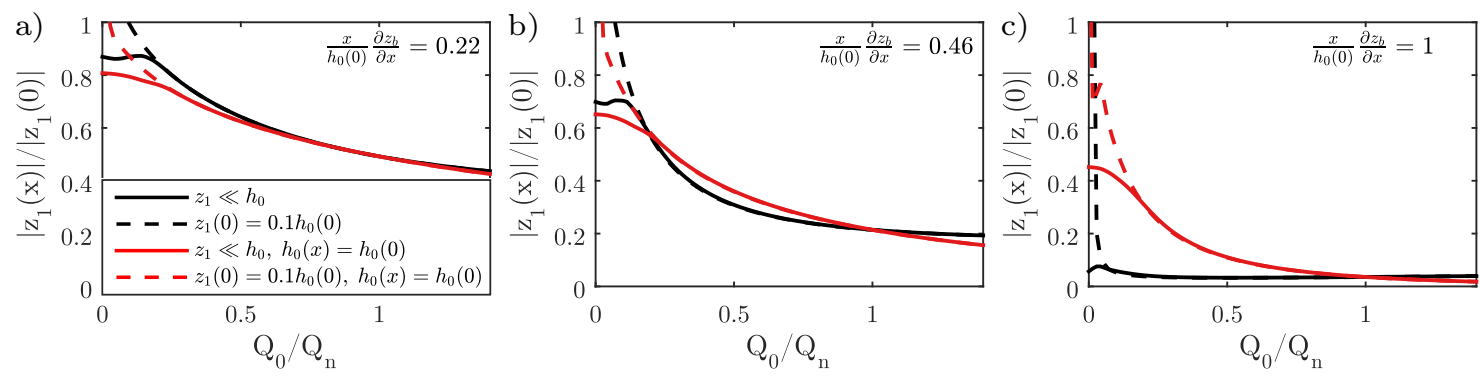

Figure 4.11: Tidal admittance for three stations located at a tidal river subject to backwater effects (black) and as predicted by a conventional model that neglects the backwater (red); dashed lines show the admittance of a wave with infinitesimal amplitude, solid lines show the admittance of a wave with finite amplitude; without backwater effects, the admittance decays exponentially, with backwater effects, the admittance decreases hyperbolically along the river

a constant depth along the river.

At a station near the sea, the tidal amplitude is always higher than predicted by the conventional model (figure 4.11a), except at normal flow, when the amplitudes are equal. At a station in the transition reach (figure $4.11 \mathrm{~b}$ ), the admittance is higher during low flow and high flow, but lower during intermediate river flow. Beyond the point where the riverbed reaches sea level (figure 4.11c), the admittance is much smaller during low flow than predicted by the conventional model, and slightly larger during high flow. In general, when the backwater dynamics are neglected, the admittance is predicted to decrease exponentially with the river discharge. In fact, the tidal amplitude decreases hyperbolically, due to the change of the tidally averaged water level. It drops more rapidly than in the exponential approximation for low river discharges, and less rapidly during high river discharge.

\subsubsection{Reflected wave}

A decomposition of the tidal wave into the incoming and the reflected parts shows that the magnitude of the reflected wave is indeed not entirely negligible (figure 4.12). The reflected wave increases the amplitude of the total wave. As the reflected wave leaves the river, the amplitude and phase of the tide at the river mouth slightly differ from that of the incoming wave (figure 4.12, inset).

The tide at the river mouth thus depends on the river geometry and river discharge. This is important for the prescription of boundary conditions. It 

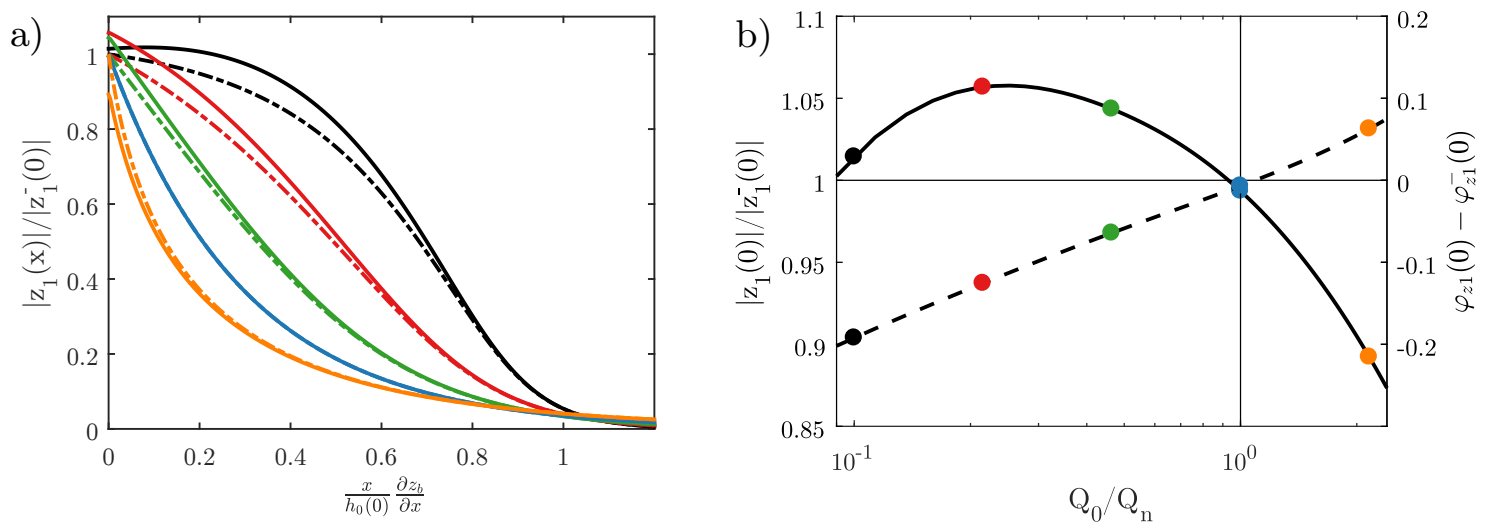

Figure 4.12: a) Admittance of the combined incoming and reflected waves $\left(\left|z_{1}(x)\right| /\left|z_{1}^{-}(0)\right|\right.$, solid) and only of the upstream travelling wave $\left(\left|z_{1}^{-}(x)\right| /\left|z_{1}^{-}(0)\right|\right.$, dash-dotted) along the river. b) amplitude (solid) and phase (dashed) of the combined wave with respect to incoming wave at the river mouth, depending on the river discharge. Scenarios as in figure 4.8

seems natural to specify the surface level $z_{s}(t, 0)$ at the river mouth, as the astronomical tide can reliably be predicted (Egbert and Erofeeva, 2002). This trivial approach is typically applied in one-dimensional models of tidal rivers that do not include the adjacent sea (Bolla Pittaluga et al., 2015b). In section 4.3.7 we apply this to approximate the propagation of the wave. However, this implicitly reduces the amplitude of the tide entering from the sea by the amplitude of the reflected wave, and thus violates causality as it implies that the astronomical tide depended on the estuarine geometry and river discharge. For the numerical computation of the tide in section 4.4 we therefore only prescribe a value for the incoming wave $\hat{z}_{1}^{-}$at the seaward boundary, and let the seaward travelling wave $\hat{z}_{1}^{+}$pass without reflection. As only one value is specified per frequency component at each boundary, we express it as a linear combination of the incoming and seaward travelling wave, according to equation (4.14b). The mean water level at the mouth also varies over a Metonic cycle (Woodworth, 2017) and due to the river discharge, but these variations are negligible.

Conversely, a trivial boundary condition of zero tidal amplitude or discharge causes reflection at the upstream boundary. This can be circumvented by placing the upstream boundary far from the sea (Godin and Martínez, 1994; Cai et al., 2014). We avoid reflection by expressing the upstream boundary condition as a linear combination of the upstream and downstream travelling waves and set only the amplitude of the downstream travelling wave to zero as $Q_{1}^{+}=0$. 

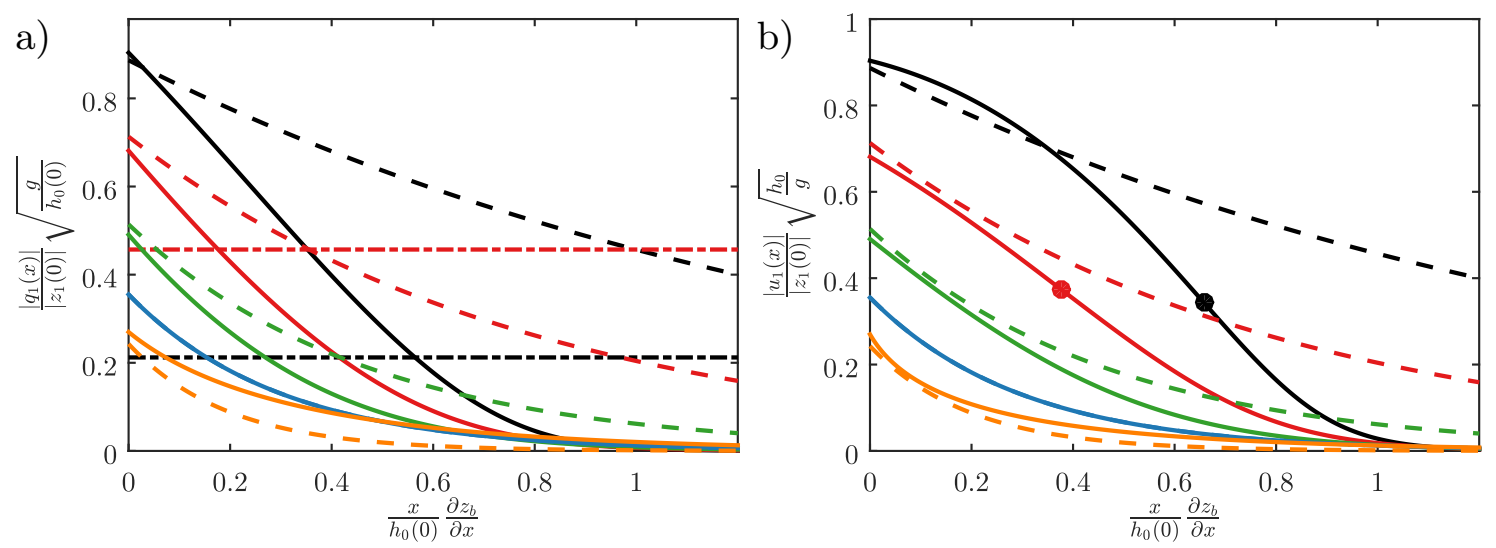

Figure 4.13: Magnitudes of tidal discharge (a) and tidal velocity (b), along a tidal river with a sloping bed (solid), and constant depth (dashed); river discharge indicated by dash-dotted line in (a); scenarios as in figure 4.8

\subsubsection{Tidal discharge}

The tidal discharge decreases with increasing river discharge (figure 4.13a). It diminishes gradually along the river so that no extrema or inflexion points occur.

As long as the river discharge is low, the tidally averaged water surface forms a backwater profile and the tidal discharge at the river mouth reduces at a higher rate, than predicted by a conventional model where the depth is constant along the river.

The most upstream point of flow reversal is located closer to the sea, compared to the conventional model. Similar to the tidal water surface amplitude, the tidal discharge amplitude is marginal beyond the point where the riverbed reaches sea level. The higher rate at which the discharge is reduced is caused by depth convergence, which, recalling equation (4.26a), has an effect on the discharge that is opposite to the effect on the tidal surface level amplitude. The lower initial discharge at the river mouth is also a direct consequence of depth convergence. Recalling the continuity equation (4.2a), the tidal discharge of the main species is:

$$
Q_{1}=\mathrm{iw} \frac{\omega}{k_{1 Q}} z_{1} .
$$

which can be directly evaluated at the river mouth in combination with the approximate wavenumber (4.16a). The tidal discharge at the river mouth is thus smaller because it is inversely proportional to the wavenumber $k_{1 Q}$, which itself is larger in magnitude, due to depth convergence. 


\subsubsection{Tidal velocity}

The cross section averaged flow velocity is directly proportional to the discharge $u=\frac{1}{A_{0}} Q$ and can thus also be explicitly evaluated at the river mouth. The tidal velocity amplitude decreases with the river discharge. However, the magnitude of the tidal velocity amplitude decreases along the river at a lower rate than the tidal discharge, when the river is below normal flow, which follows from the chain rule:

$$
k_{1 u}=-\mathrm{i} \frac{1}{u_{1}} \frac{\partial u_{1}}{\partial x}=\mathrm{i} \frac{1}{h_{0}} \frac{\partial h_{0}}{\partial x}+\mathrm{i} \frac{1}{\mathrm{w}} \frac{\partial \mathrm{w}}{\partial x}+k_{1 Q} .
$$

Width and depth convergence increase the magnitude of the velocity compared to the discharge, similar to the surface elevation amplitude, but the phase is similar to that of the discharge. An inflexion point marks the transition to the upstream reach of the tidal river, where the tidal velocity amplitude is rapidly reduced. As for the tidal surface level amplitude, this inflexion point is located the closer to the river mouth the higher the river discharge (figure 4.13). Overall, the magnitude of the tidal velocity amplitude decreases at a slightly higher rate than the magnitude of the tidal surface elevation, and the inflexion point of the velocity is located closer to the sea.

When the river flow is very low, the tidal velocity amplitudes are higher than that predicted by a conventional model along the downstream reach of a tidal river. It is lower than predicted by the conventional model when the river flow is intermediate or large. The tidal excursion length near the sea is similar but decreases more rapidly towards upstream than predicted by the conventional model (not shown). As the salinity intrusion depends on the tidal excursion (Savenije, 2012a), this implies that salinity intrudes the less far up a river the larger its bed slope, as long as the slope does not strongly change the mixing.

\subsubsection{Phase lag}

The damping of the tide is related to the phase lag $\Delta \varphi_{1}=\arg \left(u_{1}\right)-\arg \left(z_{1}\right)$ between oscillation of the water surface and velocity. When the tidally averaged water depth does not change along the river, then the damping rate and phase lag are directly proportional, as long as the damping is low, which follows from equation (4.23a):

$$
\Delta \varphi_{1}=\arctan (a) \approx a, a<1 .
$$

For strong damping $(a>1)$, the phase lag is close to $\pi / 4$, according to equation (4.23b). When the damping is low, the phase lag is zero and the tide forms a progressive wave. 
Along rivers with a sloping bed, the tidal amplitudes of the surface level and discharge change at different rates so that the tidal amplitude does not anymore decrease at a rate that is proportional to the phase lag (figure $4.9 \mathrm{~b}$ ). The phase lag is close to $\pi / 4$, even during low flow, and can even exceed $\pi / 4$ in the upstream part of the tidal river, yet downstream of the point where the bed reaches the sea level. The phase lag does also not linearly increase with the river discharge, and in the asymptotic flow reach, the phase lag even decreases with the river discharge, opposite to what occurs in a river with a horizontal bed.

\subsubsection{Overtide generation}

Friction does not only damp the tide but also generates higher frequency components, the overtide. The principal overtide advances high water and delays low water so that the tidal wave changes its shape while travelling upstream (figure 4.14a). The river discharge forces the principal overtide with twice the frequency and an amplitude proportional to the square of the main tidal species, which is evident from the frequency decomposition of the friction (equation 4.8a). If the bed slope is negligible so that the water depth remains constant along the channel, then the even overtide is smaller for lower river discharge, as $c_{2} \rightarrow 0$. However, when the riverbed is sloping, friction increases with decreasing river discharge in the upstream reach of the tidal river, as the friction is inverse proportional to depth.

When the tidally averaged width and water depth do not change along the river, then the even overtide is determined by the equation

$$
\frac{\partial^{2} Q_{2}}{\partial x^{2}}-2 \mathrm{i} r_{2} Q_{2}=-\mathrm{i} \frac{r_{1}^{2}}{\mathrm{w}} \frac{Q_{1}^{2}}{Q_{0}},
$$

which is a simplification of (4.4) and (4.8c) and in the limit where the river flow is much stronger than the tidal flow. The wavenumber $k_{1}$ is given by equation (4.21), $r_{1}$ is its imaginary part, and $k_{2}=\sqrt{2} k_{1}$ as well as $r_{2}=\sqrt{2} r_{1}$. This gives the amplitude of the overtide as:

$$
z_{2}=\frac{\omega_{1} \mathrm{w}}{8 r_{1} Q_{0}} z_{1}(0)^{2}\left(\exp \left(-2 \mathrm{i} k_{1} x\right)-\exp \left(-1 \mathrm{i} k_{2} x\right)\right),
$$

for the case when the amplitude of the overtide is zero at the river mouth. Note that even if the surface amplitude is zero at the mouth, the velocity is not, which is due to continuity (equation 4.33). Equation (4.37) is the sum of two decaying exponentials, which add to zero at the river mouth. Near the mouth the principal overtide increases at 0.6-times the rate which that the 

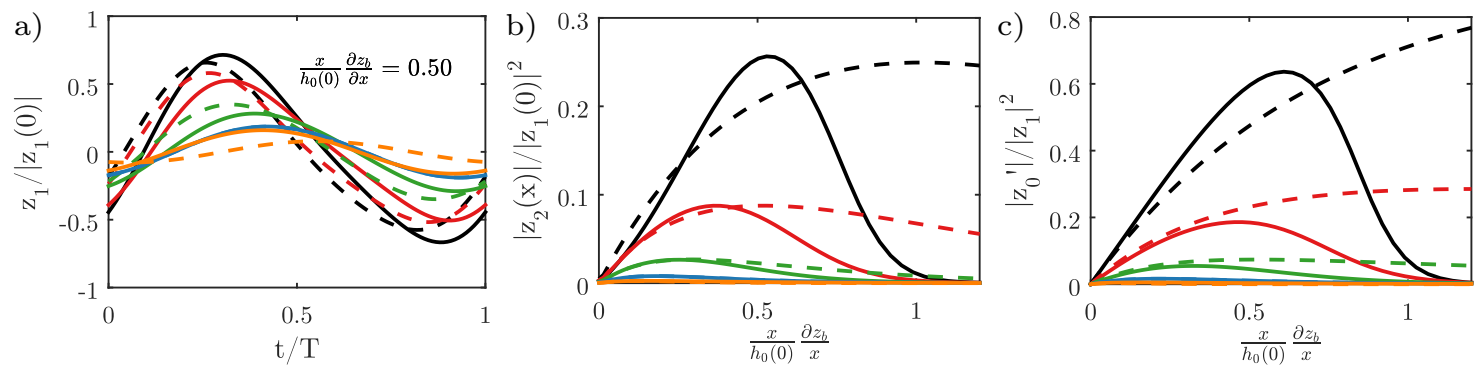

Figure 4.14: a) At a station water level variation over a tidal cycle for $z_{1}(0)=$ $\left.0.1 h_{0}(0) ; b\right)$ along river amplitude of the even overtide; c) along river subtidal water level setup; solid lines show the tide in a backwater affected river, and dashed lines show the prediction of the tide when the backwater is neglected; scenarios as in figure 4.8

amplitude of the main tidal species decreases, and far upstream it decreases at 1.4-times the rate of that of the main tidal species.

In a river with a sloping bed, the principal overtide increases near the sea, similar to the case when the tidally averaged water depth remains constant. However, in the upstream part of the tidal river, the tide is damped more rapidly when the bed is sloping. Similar to the main tidal species, the amplitude becomes marginal beyond the point where the bed level reaches sea level (figure 4.14b).

\subsubsection{Water level setup}

The tidal flow raises the tidally averaged water level by increasing friction (equation 4.3). The water level, therefore, oscillates with the tidal range over the spring-neap cycle. Such subtidal harmonics have been described by Kukulka and Jay (2003a); LeBlond (1979); Buschman et al. (2009) and Sassi and Hoitink (2013). The water level setup $z_{0}^{\prime}$ caused by the tide with respect to the water depth $h_{0}^{\prime}$ that is only due to river flow can be approximated by a perturbation expansion of the backwater equation (4.3). For dominant river flow, this is done by substituting $h_{0}^{\prime}+z_{0}^{\prime}$ for $h_{0}$, expanding the equations as a series in $z_{0}^{\prime}$ and keeping only the terms of largest magnitude:

$$
\frac{\partial z_{0}^{\prime}}{\partial x}=\frac{c_{d}}{g \mathrm{w}^{2} h_{0}^{4}}\left(\frac{1}{2}\left|Q_{1}\right|^{2} h_{0}-3 Q_{0}^{2} z_{0}^{\prime}\right) .
$$

The first term generates and the second term damps the water level setup. For a river at normal flow, where the tidally averaged water depth remains 
constant along the channel, the water level setup is:

$$
\begin{aligned}
z_{0}^{\prime} & =\frac{h_{0}}{6} \frac{r_{0}}{r_{0}-r_{1}} \frac{\left|Q_{1}(0)\right|^{2}}{\left|Q_{0}\right|^{2}}\left(\exp \left(-2 r_{0} x\right)-\exp \left(-2 r_{1} x\right)\right), \\
r_{0} & =\frac{1}{2} \frac{c_{d} Q_{0}^{2}}{g \mathrm{w}^{2} h_{0}^{4}} .
\end{aligned}
$$

where $r_{1}=\operatorname{Im}\left(k_{1, a}\right)$ is the damping rate of the main tidal species as given by equation (4.21), and $r_{0}$ is the damping rate of the mean water level setup. $Q_{1}(0)$ is the discharge at the river mouth as given by equation (4.33). The water level setup is the difference of two decaying exponentials, which are zero at the river mouth. The water level setup rises along the river twice as rapidly as the main tidal species is damped but thereafter decays at a lower rate than the main tidal species, because $r_{0} \ll r_{1}$ (figure 4.14c).

In rivers with a sloping bed, the tidally averaged water level setup is always marginal beyond the point where the bed reaches sea level (figure 4.14c). The water level setup has thus a pronounced local maximum. This maximum is lower and closer to the river mouth for higher river discharge. The rapid reduction of the water level setup in the upstream part of the tidal river is, as the reduction of the tidal amplitude, caused by the variation of the water depth with the river discharge.

Far upstream, where the flow becomes asymptotically uniform, the damping rate can be expressed as a function of the water level only, similar to that of the main tidal species (equation 4.32c):

$$
r_{0}=-\frac{1}{2} \frac{1}{h_{0}} \frac{\partial z_{b}}{\partial x}, x \rightarrow \infty .
$$

This rate is higher than that of the main tidal species (equation 4.32c) and is also higher the lower the river discharge.

The amplitude of the principal overtide and the subtidal water level setup change along the channel in a similar manner, because they are both forced by the part of the friction term that is proportional to $Q_{1}^{2}$, yet the overtide damps out more rapidly. When the main tidal species is diurnal, as in the Kapuas, then the overtide is superimposed on the semi-diurnal tide that enters the river from the sea so that the semi-diurnal tide along the river strongly depends on the river flow.

\subsection{Discussion}

In a channel with a horizontal bed, the tide would intrude thousands of kilometres upstream during low river flow (Godin and Martínez, 1994; Cai et al., 
2014). The limit of tidal intrusion observed in natural rivers is often related to dams or cataracts (Jay et al., 2015; Parker, 2007). However, a sloping bed sufficiently explains the extinction of the tide and no physical obstacle is required.

Even when the bed is horizontal, the water depth varies with the river discharge (Cai et al., 2016). Without a sloping bed, the water surface elevation forms a drawdown curve even during low flow, and the river does not asymptotically approach uniform flow. The assumption of a horizontal bed is thus not realistic. With a sloping bed, the water surface forms a backwater profile during low flow so that the tide propagates distinctly differently. This is important because the tide is strongest when the river discharge is low. Tidal rivers can be expected to be more often in a state of backwater than subject to drawdown, as their morphology is shaped by high flows, which occur infrequently.

Near the river mouth, the tidal flow is strong, which is relevant in the context of sediment transport and the intrusion of salinity. The tidal influence is particularly large in the region where the flow reverses. At some point along the river, the amplitude of the tidal discharge approximately equals the river discharge. The tidal discharge decreases more rapidly along the river for a higher bed slope, which moves the most upstream point of flow reversal, and thus the fluvial-tidal transition, closer to the sea (section 4.4.8). This will have an impact on river morphology. In the Kapuas, for example, the fluvial-tidal transition is marked by a rapid reduction of bed material grain size as well as of the bed form height (chapter 3).

In rivers with a horizontal bed, the water level setup induced by the tide reaches far upstream, where the amplitude of the main tidal species has long become insignificant (Hoitink and Jay, 2016). The point where the tidally averaged water level setup elevates the spring tidal range more than the tidal range difference between spring tide and neap tide is considered to be the upstream end of the fluvial-tidal transition (Jay et al., 2015). At this point, the lowest low water occurs at neap tide, not at spring tide anymore. In rivers with a sloping bed, the water level setup becomes marginally small beyond the point where the bed reaches sea level, thus it does not reach far upstream (section 4.4.12).

One important effect of a sloping bed is that the tidal amplitude rapidly decreases beyond the point where the bed level reaches sea level under all flow conditions. This limits the extent to which sea level rise poses a risk to upstream areas. A rise in sea level merely shifts the head of tides farther upstream, leaving the reaches beyond the point where the bed reaches the 
raised sea level unaffected. Surprisingly, the bed slope has so far not been considered to be a major factor that influences the resilience of estuaries and rivers with respect to sea level rise (Prandle and Lane, 2015).

Although the land adjoined to those reaches can be at risk of overland flooding during storm surges in gently sloping coastal lowlands (Frazier et al., 2010). In our idealized analysis, we keep the drag coefficients constant. In practice, bed the roughness varies with the tidal and river discharge in a complex manner (van Rijn, 1984a). As the idealized model still reproduces the observations of the river tide in the Kapuas, we conclude that the variation of roughness is less important than that of the water depth and that therefore the bed slope is a more important factor to consider than varying roughness. This notwithstanding, changes of roughness can be relevant for operational flood forecasting (Reef et al., 2018).

The backwater dynamics explain the tidal admittance in the Kapuas, where the tidal amplitude does not decay exponentially during low flow as predicted by conventional models of river tides. Waves joining from the subordinary distributaries may partially increase the tidal amplitude in the main channel, the Kapuas Besar. However, this causes a jump of the amplitude at the bifurcations, and thus cannot explain the observed gradual deviation from an exponential decay. A small jump in amplitude can be observed at the bifurcation of the largest side distributary, the Kapuas Kecil. This is in line with the theory of transmission and reflection at bifurcations (Lighthill, 2001), according to which a small channel that joins a larger one only exerts a small influence on the latter. In turn, the Kapuas Besar strongly forces the subordinate distributaries. Backwater dynamics are thus the most likely cause for the observed low flow admittance in the Kapuas River.

The estuarine geometry strongly influences how the tide propagates (Friedrichs, 2010). We analyze the tidal propagation along a river with idealized geometry, as this reveals the general effects of a sloping bed and allows for a mathematical explanation. Our findings are relevant for rivers that do not converge in width, and where depth converges approximately linearly during low flow (section 4.4.1). This is different from tide-dominated estuaries, where the width converges exponentially and where the tidally averaged water level varies only slightly between the seasons (Savenije et al., 2008; van Rijn, 2011; Garel and Cai, 2018). The bed slope in our models is constant, whereas, on basin scale, the slope decreases along rivers in the downstream direction (Richards, 1982). A constant slope approach can be justified based on the results of Seminara et al. (2012), who showed the bed slope is approximately constant along tidal rivers in morphological equilibrium. 


\subsection{Conclusion}

Our observations from the Kapuas River show that the tide propagates distinctly differently during low flow than expected from idealized models that ignore the bed slope. Rather than decreasing exponentially in amplitude and intruding far upstream, the tide remains isosynchronous near the sea, and then rapidly diminishes where the bed reaches sea level. Conventionally, tidal rivers are conceptualized as non-converging channels with horizontal bed, where the tidally averaged width and depth neither change along channel nor over time. However, the tidally averaged water depth remains only constant near the sea, and the bed rises along the river. Farther upstream, the depth thus varies with the river discharge so that the water surface forms a backwater profile during low flow. This is relevant because tides are strongest during low flow. We extend the idealized model of tidal rivers to include the effects of backwater. Our idealized model shows that the backwater profile considerably influences how the tide propagates during low flow. The principal effect is a larger than expected tidal range near the sea, due to convergence of the depth, as well as a lower than expected tidal range far upstream, due to lower depth and higher friction. Tidal intrusion is effectively limited to the point where the bed reaches sea level. This also applies to the overtide and the tidally averaged water level setup. Our extended model does not only explain the observed tidal dynamics in the Kapuas River but resembles, in general, a more comprehensive model of river tides, since variation of the river discharge and a sloping bed are common to all rivers.

\section{Appendix}

\subsection{Transformation the shallow water into the wave equation}

The cross-sectionally shallow water equation (4.2a) and (4.2b) relies on the following assumptions

- hydrostatic flow,

- rectangular cross section without tidal flats,

- straight channel,

- negligible side inflow,

- negligible variation of velocity within the cross section (unit Boussinesq coefficient),

- negligible Coriolis force, 
- negligible density gradients.

The following steps transform the shallow water equation into the wave equation (4.4):

1 rewrite the momentum equation (4.2b) in its reduced form:

$$
\frac{\partial Q}{\partial t}+\frac{\partial}{\partial x}\left(\frac{Q}{A^{2}}\right)+g A \frac{\partial z_{s}}{\partial x}+c_{d} \frac{|Q| Q}{A \mathrm{R}_{\mathrm{h}}}=0
$$

2 ignore advective acceleration $\left(\frac{\partial}{\partial x} \frac{Q}{A} \ll \frac{\partial Q}{\partial t}\right)$,

3 divide the momentum equation by $A$.

4 approximate the hydraulic radius for a wide channel $R_{h} \rightarrow h$,

$5 A \rightarrow \mathrm{w} h$,

6 expand $1 / h^{p}$ as truncated Taylor series $\left(1 / h^{p} \rightarrow 1 / h_{0}^{p}\right)$,

$7 h \rightarrow h_{0}+z$, where $h_{0}=z_{0}-z_{b}$,

8 differentiate the continuity equation with respect to $x$,

9 divide the continuity equation by $\mathrm{w}$,

10 differentiate the momentum equation with respect to $t$,

11 divide the momentum equation by $g$,

12 eliminate $z$ by subtracting the continuity from the momentum equation. The result is the wave equation (4.4).

\subsection{Friction term}

This section derives the frequency components (equations (4.8a)-(4.8b))) of the Chebyshev approximation of the signed square $|Q| Q$ of the friction term (equation 4.6). The products and powers of complex exponentials are reduced by the following identities:

$$
\begin{aligned}
\operatorname{Re}\left\{c_{1} e^{\mathrm{i} \omega_{1} t}\right\} \operatorname{Re}\left\{c_{2} e^{\mathrm{i} \omega_{2} t}\right\}= & \frac{1}{2} \operatorname{Re}\left\{c_{1} c_{2} e^{\mathrm{i}\left(\omega_{1}+\omega_{2}\right) t}\right\} \\
& +\frac{1}{2} \operatorname{Re}\left\{c_{2} c_{1}^{*} e^{\mathrm{i}\left(\omega_{2}-\omega_{1}\right) t}\right\}, \\
\operatorname{Im}(c)= & \operatorname{Re}\{-\mathrm{i} c\}, \\
c c^{*}= & |c|^{2} .
\end{aligned}
$$

Where the asterisk * denotes the complex conjugate. The expansion of the discharge $Q$ up for the two leading non-zero frequency components $\left(\omega_{j}=j \omega_{1}\right)$ 
yields:

$$
\begin{aligned}
F= & f_{0} Q_{h r}{ }^{2}+f_{1}\left(Q_{0} Q_{h r}+\operatorname{Re}\left\{e^{\omega_{1} t 1 \mathrm{i}} Q_{1}\right) Q_{h r}+\operatorname{Re}\left\{e^{\omega_{1} t 2 \mathrm{i}} Q_{2}\right) Q_{h r}\right) \\
& +f_{2}\left(\left|Q_{0}\right| Q_{0}+2 Q_{0} \operatorname{Re}\left\{e^{\omega_{1} t 1 \mathrm{i}} Q_{1}\right)\right. \\
& +2 Q_{0} \operatorname{Re}\left\{e^{\omega_{1} t 2 \mathrm{i}} Q_{2}\right)+\operatorname{Re}\left\{e^{\omega_{1} t 1 \mathrm{i}} Q_{1}\right)^{2} \\
& \left.+2 \operatorname{Re}\left\{e^{\omega_{1} t 1 \mathrm{i}} Q_{1}\right) \operatorname{Re}\left\{e^{\omega_{1} t 2 \mathrm{i}} Q_{2}\right)+\operatorname{Re}\left\{e^{\omega_{1} t 2 \mathrm{i}} Q_{2}\right)^{2}\right\} .
\end{aligned}
$$

With reduced powers, this is:

$$
\begin{aligned}
F= & f_{0} Q_{h r}{ }^{2}+f_{1}\left(Q_{0} Q_{h r}+Q_{1} Q_{2} e^{\omega_{1} t 2 \mathrm{i}}+\operatorname{Re}\left\{e^{\omega_{1} t 1 \mathrm{i}} Q_{1}\right) Q_{h r}+\operatorname{Re}\left\{e^{\omega_{1} t 2 \mathrm{i}} Q_{2}\right) Q_{h r}\right) \\
& +f_{2}\left(\left|Q_{0}\right| Q_{0}+2 Q_{0} \operatorname{Re}\left\{e^{\omega_{1} t 1 \mathrm{i}} Q_{1}\right)+2 Q_{0} \operatorname{Re}\left\{e^{\omega_{1} t 2 \mathrm{i}} Q_{2}\right)\right. \\
& +\frac{1}{2}\left(\left|Q_{1}\right|^{2}+\operatorname{Re}\left(Q_{1}^{2} e^{\omega_{1} t 2 \mathrm{i}}\right)\right)+\left(Q_{2} Q_{1}^{*} e^{\omega_{3} t 1 \mathrm{i}}+\frac{1}{2}\left(\left|Q_{2}\right|^{2}+\operatorname{Re}\left(Q_{2}^{2} e^{\omega_{4} t \mathrm{i}}\right)\right)\right) .
\end{aligned}
$$

$F_{0}$ (equation $\left.4.8 \mathrm{a}\right)$ is the mean component $\left(\omega_{0}=0\right)$ of this expression. Similarly for the time derivative $F^{\prime}$ :

$$
\begin{aligned}
F^{\prime}= & f_{1} \omega_{1}\left(\operatorname{Re}\left\{\mathrm{i} e^{\mathrm{i} \omega_{1} t} Q_{1}\right\} Q_{h r}+2 \operatorname{Re}\left\{\mathrm{i} e^{2 \mathrm{i} \omega_{1} t} Q_{2}\right\} Q_{h r}\right) \\
& +2 f_{2} \omega_{1}\left(+Q_{0} \operatorname{Re}\left\{\mathrm{i} e^{\mathrm{\omega} t 1 \mathrm{i}} Q_{1}\right\}+2 Q_{0} \operatorname{Re}\left\{\mathrm{i} e^{\omega_{1} t 2 \mathrm{i}} Q_{2}\right\}\right. \\
& +\operatorname{Re}\left\{e^{\mathrm{i} \omega_{1} t} Q_{1}\right\} \operatorname{Re}\left\{\mathrm{i} e^{\mathrm{i} \omega_{1} t} Q_{1}\right\}+\operatorname{Re}\left\{e^{2 \mathrm{i} \omega_{1} t} Q_{2}\right\} \operatorname{Re}\left\{\mathrm{i} e^{\mathrm{i} \omega_{1} t} Q_{1}\right\} \\
& \left.+2 \operatorname{Re}\left\{e^{\mathrm{i} \omega_{1} t} Q_{1}\right\} \operatorname{Re}\left\{\mathrm{i} e^{2 \mathrm{i} \omega_{1} t} Q_{2}\right\}+2 \operatorname{Re}\left\{e^{2 \mathrm{i} \omega_{1} t} Q_{2}\right\} \operatorname{Re}\left\{\mathrm{i} e^{2 \mathrm{i} \omega_{1} t} Q_{2}\right\}\right) \\
= & f_{1} \omega_{1}\left(\operatorname{Re}\left\{\mathrm{i} e^{\mathrm{i} \omega_{1} t} Q_{1}\right\} Q_{h r}+2 \operatorname{Re}\left\{\mathrm{i} e^{2 \mathrm{i} \omega_{1} t} Q_{2}\right\} Q_{h r}\right) \\
& +f_{2} \omega_{1}\left(\operatorname{Re}\left\{\mathrm{i}\left|Q_{1}\right|^{2}\right\}+\operatorname{Re}\left\{\mathrm{i} Q_{1}^{2} e^{\mathrm{i} \omega_{2} t}\right\}-\left(\operatorname{Re}\left\{\mathrm{i} Q_{2} Q_{1}^{*} e^{\mathrm{i} \omega_{1} t}\right\}\right.\right. \\
& \left.+\operatorname{Re}\left\{-\mathrm{i} Q_{1} Q_{2} e^{\mathrm{i} \omega_{3} t}\right\}\right)+2\left(\operatorname{Re}\left\{\mathrm{i} Q_{2} Q_{1}^{*} e^{\mathrm{i} \omega_{1} t}\right\}+\operatorname{Re}\left\{\mathrm{i} Q_{1} Q_{2} e^{\mathrm{i} \omega_{3} t}\right\}\right) \\
& +2\left(\operatorname{Re}\left\{\mathrm{i}\left|Q_{2}\right|^{2}\right\}+\operatorname{Re}\left\{\mathrm{i} Q_{2}^{2} e^{\mathrm{i} \omega_{4} t}\right\}\right)+2 Q_{0} \operatorname{Re}\left\{\mathrm{i} e^{\mathrm{i} \omega_{1} t} Q_{1}\right\} \\
& \left.+4 Q_{0} \operatorname{Re}\left\{\mathrm{i} e^{\mathrm{i} \omega_{2} t} Q_{2}\right\}\right)
\end{aligned}
$$

$F_{1}^{\prime}$ and $F_{2}^{\prime}$ (equations (4.8b) and (4.8b)) are complex amplitudes of the components with frequencies $\omega_{1}$ and $\omega_{2}$, respectively. 


\subsection{Friction coefficients}

The coefficients of the quadratic Chebyshev polynomial (equation 4.6), determined with the method of Dronkers (1964), are:

$$
\begin{aligned}
\alpha=\min & \left(1, \max \left(-1, \frac{Q_{\max }+Q_{\min }}{Q_{\max }-Q_{\min }}\right)\right), \\
f_{0}=-( & \left(\frac{3}{2} \sin (2 \alpha)+(2+\cos (2 \alpha))\left(\frac{1}{2}-\frac{\alpha}{\pi}\right)\right) \\
& \quad+\frac{1}{\pi}\left(\cos (2 \alpha)\left(\frac{\pi}{2}-\alpha+\frac{2}{3} \sin (2 \alpha)-\frac{1}{12} \sin (4 \alpha)\right)\right) \\
& \left.\quad-\frac{1}{\pi}\left(\cos (\alpha)\left(\frac{1}{3} \sin (3 \alpha)+3 \sin (\alpha)+\cos (\alpha)(2 \pi-4 \alpha)\right)\right)\right), \\
f_{1}=-\frac{1}{\pi} & \left(\left(\frac{1}{3} \sin (3 \alpha)+3 \sin (\alpha)+\cos (\alpha)(2 \pi-4 \alpha)\right)\right. \\
& \left.\quad-\left(4 \cos (\alpha)\left(\frac{\pi}{2}-\alpha+\frac{2}{3} \sin (2 \alpha)-\frac{1}{12} \sin (4 \alpha)\right)\right)\right), \\
f_{2}=-\frac{1}{\pi} & \left(\pi-2 \alpha+\frac{4}{3} \sin (2 \alpha)-\frac{1}{6} \sin (4 \alpha)\right) .
\end{aligned}
$$

\subsection{Wave propagation for second-order ordinary differential equa- tions with variable coefficients}

When a wave propagates across a sudden change of the cross section geometry, its amplitude and wavelength change and a reflected wave occurs (Schönfeld, 1951; Lighthill, 2001; Ippen, 1966). This paragraph shows that the propagation of a wave along a gradually varying channel can be understood as a wave travelling across a sequence of infinitesimal steps.

A second order ordinary differential equation has the form

$$
c_{2}(x) y^{\prime \prime}(x)+c_{1}(x) y^{\prime}(x)+c_{0}(x) y(x)=s(x) .
$$

The solution is the superposition of two waves, one that travels to the left and one that travels to the right: $y=y^{-}+y^{+}$. When the coefficients $c_{0,1,2}$ are constant, then these waves travel as $y^{ \pm}=y^{ \pm}(0) \exp \left(r^{ \pm} x\right)$, where $r^{ \pm}$are the roots of the characteristic polynomial $c_{2} r^{2}+c_{1} r+c_{0}=0$. When the coefficients $c_{0,1,2}$ vary in space, then the solution can be approximated by partitioning the domain into segments over which the coefficients are kept constant. In the limit where the length of each segment becomes infinitesimal, the approximation is exact. Along a segment, the wave travels as

$$
y_{l}=\hat{y}_{l}^{-} \exp \left(r_{l}^{-} x\right)+\hat{y}_{l}^{+} \exp \left(r_{l}^{+} x\right),
$$


where $l$ is the segment index. In the case of constant coefficients $\hat{y}_{l}^{ \pm}=y^{ \pm}(0)$. When the coefficients vary in space, then the $\hat{y}_{l}^{ \pm}$differ between the segments. To determine how the $\hat{y}_{l}^{ \pm}$change, consider two segments of length $\Delta x$ that border each other at $x_{0}$ (figure 4.15).

Solutions to (4.7a) are twice differentiable. Therefore at the border $x_{0}$ between two segments:

$$
\begin{gathered}
y_{l}\left(x_{0}\right)=y_{l+1}\left(x_{0}\right), \\
\frac{\partial y_{l}}{\partial x}\left(x_{0}\right)=\frac{\partial y_{l+1}}{\partial x}\left(x_{0}\right) .
\end{gathered}
$$

or in matrix form

$$
\left[\begin{array}{cccc}
1 & 0 & 0 & 0 \\
1 & 1 & -1 & -1 \\
r_{l}^{-} & r_{l}^{+} & -r_{l+1}^{-} & -r_{l+1}^{+} \\
0 & 0 & 0 & 1
\end{array}\right]\left[\begin{array}{c}
\hat{y}_{l}^{-} \\
\hat{y}_{l}^{+} \\
\hat{y}_{l+1}^{-} \\
\hat{y}_{l+1}^{+}
\end{array}\right]=\left[\begin{array}{c}
y^{-}\left(x_{0}-\Delta x\right) \\
0 \\
0 \\
y^{+}\left(x_{0}+\Delta x\right)
\end{array}\right]
$$

When there is only one incoming wave, i.e. when either $y^{-}\left(x_{0}-\Delta x\right)$ or $y^{+}\left(x_{0}-\right.$ $\Delta x$ ) are zero, there occurs only transmission and reflection of a single wave. For waves travelling to the left, the reflection and transmission coefficients are $T^{-}=\frac{\hat{y}_{l+1}^{-}}{\hat{y}_{l}^{-}}=1-\frac{r_{l+1}^{-}-r_{l}^{-}}{r_{l}^{+}-r_{l+1}^{-}}$and $R^{-}=1-T^{-}=\frac{\hat{y}_{l}^{+}}{\hat{y}_{l}^{-}}=\frac{r_{l+1}^{-}-r_{l}^{-}}{r_{l}^{+}-r_{l+1}^{-}}$. For waves travelling to the right these are $T^{+}=\frac{\hat{y}_{l}^{+}}{\hat{y}_{l+1}^{+}}=1+\frac{r_{l+1}^{+}-r_{1}^{+}}{r_{l}^{+}-r_{l+1}^{-}}$and $R^{+}=1-T^{+}=$ $\frac{\hat{y}_{l+1}^{-}}{\hat{y}_{l+1}^{+}}=\frac{r_{l+1}^{+}-r_{l}^{+}}{r_{l}^{+}-r_{l+1}^{-}}$.

The wave $y^{-}$thus changes while travelling from the centre of the left to the centre of the right segment as

$$
\begin{aligned}
y^{-}\left(+\frac{\Delta x}{2}\right)= & y^{-}\left(-\frac{\Delta x}{2}\right) \exp \left(+r_{l}^{-} \frac{\Delta x}{2}\right) T^{-} \exp \left(+r_{l+1}^{-} \frac{\Delta x}{2}\right) \\
& +y^{+}\left(+\frac{\Delta x}{2}\right) \exp \left(-r_{l+1}^{+} \frac{\Delta x}{2}\right) R^{+} \exp \left(+r_{l+1}^{-} \frac{\Delta x}{2}\right),
\end{aligned}
$$

where the first term is the transmitted part of the wave that comes from the left $y^{-}$and the second term is the reflected part of the wave $y^{+}$that comes from the right. This is visualised in figure 4.15.

When the segment length is infinitesimal so that $\exp \left(r^{ \pm} \Delta x\right)=1+r^{ \pm} \Delta x+$ $\mathrm{O}\left(\Delta x^{2}\right)$, and the coefficients $c_{0,1,2}$ are smooth functions so that $r_{l+1}^{ \pm}=r_{l}^{ \pm}+$ $\Delta x \frac{\partial r^{ \pm}}{\partial x}+\mathrm{O}\left(\Delta x^{2}\right)$, then the right going wave is described by the first order 


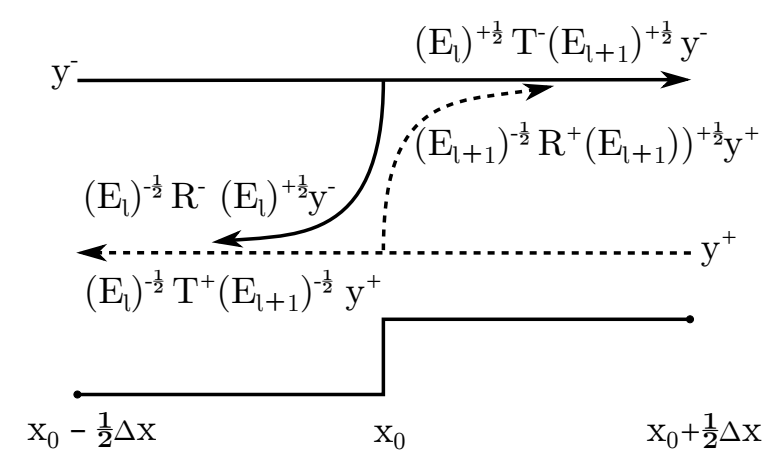

Figure 4.15: Development of the left $y^{+}$and right $y^{-}$wave in the case of variable coefficients

ordinary differential equation:

$$
\begin{aligned}
\frac{\partial y^{-}}{\partial x} & =\lim _{\Delta x \rightarrow 0}\left(\frac{1}{\Delta x}\left(y^{-}\left(\frac{\Delta x}{2}\right)-y^{-}\left(-\frac{\Delta x}{2}\right)\right)\right) \\
& =(r^{-}+\underbrace{\frac{-1}{r^{-}-r^{+}} \frac{\partial r^{-}}{\partial x}}_{T^{-}}) y^{-}+\underbrace{\frac{-1}{r^{-}-r^{+}} \frac{\partial r^{+}}{\partial x}}_{R^{-}} y^{+} .
\end{aligned}
$$

Similarly, the wave $y^{+}$travelling to the right develops as

$$
\begin{aligned}
y^{+}\left(-\frac{\Delta x}{2}\right)= & y^{+}\left(+\frac{\Delta x}{2}\right) \exp \left(-r_{l+1}^{+} \frac{\Delta x}{2}\right) T^{+} \exp \left(-r_{l}^{+} \frac{\Delta x}{2}\right) \\
& +y^{-}\left(-\frac{\Delta x}{2}\right) \exp \left(+r_{l}^{-} \frac{\Delta x}{2}\right) R^{-} \exp \left(-r_{l}^{+} \frac{\Delta x}{2}\right), \\
\frac{\partial y^{+}}{\partial x}= & (+r^{+}+\underbrace{\frac{+1}{r^{-}-r^{+}} \frac{\partial r^{+}}{\partial x}}_{T^{+}}) y^{+}+\underbrace{\frac{+1}{r^{-}-r^{+}} \frac{\partial r^{-}}{\partial x}}_{R^{+}} y^{-} .
\end{aligned}
$$

The waves $y^{+}$and $y^{-}$experience the opposite effects due to a change of coefficients as mass and energy are conserved. 


\section{Separating the sensed from the unsensed fraction when inferring suspended sediment concentrations from mono-frequency acoustic backscatter}

Urrogate measurements of suspended sediment with acoustic Doppler $\checkmark$ current profilers (ADCPs) have become increasingly popular, as they require little effort and provide a high spatial and temporal resolution. However, the relation between sediment concentration and acoustic backscatter is ambiguous, as changes of the sediment concentration cannot be distinguished from changes of the particle size. Each field site and flood stage therefore requires a laborious calibration of the backscatter sensitivity with water samples. There exist various slightly different methods to infer suspended sediment concentration from the acoustic backscatter. The methods typically determine the total sediment concentration. Although widely applied, we argue that such an approach is meaningless when the suspended sediment is a mixture of sand, silt and clay, as most of the backscatter is caused by the coarsest fraction of the suspended sediment. We therefore propose a method that is explicit in inferring only the course-grained fraction, which typically dominates ADCP backscatter. Along with the improved method, we provide an easy to use and robust statistical method for selecting the best backscatter inversion model as well as for evaluating uncertainties. We illustrate the method by applying it to determine the suspended sediment concentration in the tidally influenced part of the Kapuas river, West Kalimantan, Indonesia.

This chapter is based on: Kästner, K., and A. J. F. Hoitink, Separating the sensed from the unsensed fraction when inferring suspended sediment concentrations from mono-frequency acoustic backscatter, Submitted to Water Resources Research, 2019b 


\subsection{Introduction}

Suspended sediment transport is a key control governing the morphodynamics of rivers and deltas (Syvitski and Saito, 2007). The measurement of sediment transport in large rivers is challenging. The sheer size of the river, as well as the spatial and temporal variation of the sediment concentration therein, make it infeasible to reliably determine the transport directly from water samples. A viable option is to infer the sediment concentration from acoustic backscatter (Gray and Gartner, 2009), which can be measured simultaneously with the velocity by Acoustic Doppler Current Profilers (ADCPs). Acoustics were first applied to monitor sediment fluxes in the coastal and oceanic bottom boundary layer with rigidly deployed profilers (Varadan et al., 1985; Lynch et al., 1991; Schaafsma et al., 1997; Holdaway et al., 1999; Gartner, 2004). An overview is given by Thorne and Hanes (2002). Rigidly deployed horizontal velocity profiles have more recently been used to monitor sediment transport in rivers (Moore et al., 2012; Topping et al., 2007; Wright et al., 2010; Buschman et al., 2013; Haught et al., 2017). When mounted to a vessel, ADCPs can measure the sediment flux through an entire cross-section in great detail, which has been demonstrated in a river and in several estuarine environments (Kim and Voulgaris, 2003; Hoitink, 2004; Kostaschuk et al., 2004; Wall et al., 2006; Szupiany et al., 2009; Latosinski et al., 2014; Guerrero and Lamberti, 2011; Sassi et al., 2013; Venditti et al., 2016; Sahin et al., 2017).

The suitability of acoustic backscatter for the measurement of the suspended sediment depends on the particular sedimentary characteristics of each field site. Furthermore, it requires an extensive calibration with reference samples (Guerrero and Lamberti, 2011; Sassi et al., 2012). This is because acoustic backscatter depends both on the sediment concentration as well as the size of the suspended particles. A variation of the size distribution makes the sediment concentration inferred from mono-frequency backscatter ambiguous. Changes in the particle size distribution can be distinguished from changes of the sediment concentration by measuring the backscattered sound at several frequencies. However, off-the-shelf available ADCPs operate only at a single frequency. Attenuation due to high concentrations increases the ambiguity further and reduces the maximum profiling depth (Sassi et al., 2012).

In this technical note, we introduce an adjusted approach to determine the concentration of suspended sand from single frequency acoustic backscatter in Section 5.2. Section 5.3 applies the method to data collected in the tidally influenced Kapuas River, West Kalimantan, Indonesia. Section 5.4 discusses 
the main results and section 5.5 draws general conclusions.

\subsection{Relation between suspended sediment and acoustic backscatter}

\subsubsection{Acoustic theory}

An ADCP measures the flow velocity on hand of the frequency shift of sound that is reflected, i.e. scattered back, by sediment particles moving with the flow (Gordon, 1989). The intensity of the reflected sound is related to the concentration of the suspended sediment. The intensity of the sound received by the $\operatorname{ADCP}\left(I_{r}\right)$ is given by the sonar equation:

$$
I_{r}=I_{0} \frac{1}{r^{2}} k_{s}^{2} c_{m} \exp \left(0.23\left(-2 \int_{0}^{r} \alpha_{w}+\xi c_{m} \mathrm{~d} r^{\prime}+C\right)\right),
$$

where $I_{0}$ is a reference intensity, $r$ the distance to the transducer in $\mathrm{m}, k_{s}^{2}$ the backscatter sensitivity in $\mathrm{m}^{2} / \mathrm{kg}, c_{m}$ the mass concentration of sediment in $\mathrm{kg} / \mathrm{m}^{3}, \alpha_{w}$ the attenuation by water in $\mathrm{m}^{-1}(\mathrm{~dB})$, and $\xi$ is the normalized coefficient for attenuation by sediment in $\mathrm{m}^{2} / \mathrm{kg}(\mathrm{dB})$. $C$ accounts for instrumentspecific properties at the moment of transmission. The factor $0.23 \approx \frac{\ln 10}{10}$ converts decibel to natural units $\left(10^{x / 10}=\exp 0.23 x\right)$. The intensity level is computed from the manufacturer specific raw data stored by the ADCP and instrument specific parameters (Deines, 1999).

The attenuation of water can be determined with an empirical formula such as that introduced by Ainslie and McColm (1998). Equation 5.1 neglects multiple scattering, which is only relevant for very high concentrations. It also assumes the scattering to be incoherent. According to the criteria of Merckelbach and Ridderinkhof (2006), scattering in strong flow can become partially coherent, but we consider this here to be negligible compared to other sources of errors.

The sediment concentration is found by rearranging the sonar equation as in:

$$
c_{m}=\frac{1}{k_{s}^{2}} \beta \exp \left(-0.23 \int_{0}^{r} 2 \xi c_{m} \mathrm{~d} r^{\prime}\right),
$$

where $\beta$ in $(1 / \mathrm{m})$ is the intensity ratio corrected for spherical spreading and attenuation by water.

As the backscatter sensitivity and attenuation by sediment depend on the particle size distribution of the suspended sediment, there are three unknowns: $c_{m}, k_{s}$, and $\xi$. The concentration and as well as the particle size distribution specific coefficients $\xi$ and $k_{s}$ can only be determined independently 
with multi-frequency measurements (Guerrero et al., 2013). When the size distribution does not vary strongly in space and time, then $k_{s}$ and $\xi$ can be fixed as parameters. They can be calibrated with reference concentrations of water samples (Sassi et al., 2012).

The backscatter sensitivity $1 / k_{s}^{2}$ depends on the particle size distribution of the suspended sediment. It can be determined analytically for spheres with a known diameter (Anderson, 1950). The backscatter sensitivity of natural sediment is slightly different, as the particles have an irregular shape. The relation between backscatter sensitivity and particle size is therefore typically determined empirically (Thorne and Hurther, 2014). Empirical sensitivities differ up to a factor of two for geometric scattering and even more at the transition between Raleigh and geometric scattering. For Raleigh scattering, the empirical relations are closer to each other. Typical suspended sediment in sand bed rivers like the Kapuas scatters sound at $1.2 \mathrm{MHz}$ in the Raleigh regime, as the product of the particle radius and wave number is smaller than unity.

Following Thorne and Meral (2008), the backscatter sensitivity in the Raleigh regime for particles of diameter $D$ in $\mathrm{m}$ is:

$$
k_{s}^{2} \approx\left(2.7 \frac{m^{5}}{k g}\right) 10^{9} D^{-3}, D \ll 4 \cdot 10^{-4} \mathrm{~m} .
$$

The joint backscatter sensitivity of a suspension of particles of mixed size, $\bar{k}_{s}^{2}$, is determined by convolution with the volume size distribution $p$, i.e. $\bar{k}_{s}^{2}=$ $\int_{0}^{\infty} p k_{s}^{2} \mathrm{~d} D$. Due to the cubic dependence of the backscatter sensitivity on the particle size, the largest particles scatter most of the sound, while small particles are de-facto inaudible.

Sound is attenuated by sediment due to shadowing by large particles, like sand grains, and viscous absorption by small particles classified as silt or clay. Following again Thorne and Meral (2008), the normalized attenuation coefficient $\xi$ for a $1.2 \mathrm{MHz}$ transducer is:

$$
\xi_{s} \approx 6.0 \cdot 10^{-7} D^{-1}+\left(6.6 \cdot 10^{9} \frac{1}{m^{2}}\right) D^{3}, 10^{-6} \mathrm{~m}<D<4 \cdot 10^{-4} \mathrm{~m} .
$$

Values of $\xi_{s}$ attain a minimum for a diameter near $100 \mu \mathrm{m}$ and are large for very fine and very large particles. Thus, even though fine particles do not scatter strongly, they can considerably attenuate the sound. The concentration of fine sediment can be determined from the attenuation of sound along horizontal profiles, when the concentration is high (Hanes, 2012; Guerrero and Di Federico, 2018). When the sediment concentration is not high, then 
the sound intensity is primarily determined by the backscatter, and not by the attenuation due to sediment.

\subsubsection{Backscatter inversion model}

We base our method to determine the sediment concentration from ADCP backscatter on that applied by Sassi et al. (2012) in the Mahakam, which had been originally proposed by Lee and Hanes (1995):

$$
c_{m}=\frac{\beta}{K-0.23 \xi \int_{r_{0}}^{r} 2 \beta \mathrm{d} r^{\prime}},
$$

Herein, $K=\beta_{0} / c_{m, 0}$ is a reference value for the distance $r_{0}$ from the transducer. Sassi et al. (2012) determined $K$ by the empirical relation:

$$
K=a \beta_{0}^{b} .
$$

We choose the reference point to coincide with the position of the transducer. In this case $\mathrm{a}=k_{s}^{2}$ and $\mathrm{b}=0$, since the backscatter is linear in the concentration according to equation (5.2). This simplifies the expression to:

$$
c_{m}=\frac{1}{k_{s}^{2}} \frac{\beta}{1+0.23 \frac{\xi}{k_{s}^{2}} \int_{0}^{r} 2 \beta \mathrm{d} r^{\prime}} .
$$

When sand is present, then silt, and clay contribute only a negligible fraction to the total backscatter, as the backscatter sensitivity $1 / k_{s}^{2}$ of silt and clay particles is several orders of magnitude smaller than that of sand.

Fine particles are typically carried as wash load and their concentration varies relatively little in time and space, compared to that of sand. We therefore only directly infer the fraction of the coarse sediment $\left(c_{c}\right)$ from the backscatter, and introduce the offset $c_{f}$, to account for the concentration of fine sediment, as well as the coefficient $\alpha_{f}$ to account for the attenuation by fine sediment. The total concentration $\tilde{c}$ is thus:

$$
\begin{aligned}
\tilde{\beta} & =\beta \exp \left(0.46 \alpha_{f} r\right), \\
\tilde{c}_{m} & =\underbrace{\frac{1}{k_{s}^{2}} \frac{\tilde{\beta}}{1+0.23 \frac{\xi}{k_{s}^{2}} \int_{0}^{r} 2 \tilde{\beta} \mathrm{d} r^{\prime}}}_{c_{c}}+c_{f},
\end{aligned}
$$

where $\tilde{\beta}$ is the backscatter intensity corrected for attenuation by fine sediment. Although the method separates coarse from fine sediment, the model parameters can be determined directly from backscatter and reference concentrations, without explicit information on the particle size distribution. 


\subsubsection{Model parameter selection}

Equation (5.7) corrects for attenuation by coarse sediment, and equation (5.8a) corrects additionally for a background concentration, as well as for attenuation by fines. Not all corrections are necessarily meaningful, depending on the (probably unknown) particle size distribution. The best suitable model can be selected by comparing the goodness of fit of alternative parametrizations. We determine the standard errors and confidence intervals by bootstrapping. The bootstrap confidence intervals are non-parametric and do not require the regression residuals to be normally distributed. For bootstrapping, the data is repeatedly randomly resampled with replacement. For each repetition, we fit the parameters by minimizing the sum of squared residuals, for the non-linear parametrizations we use the Levenberg-Marquardt algorithm. We average the backscatter associated with each sample over two minutes. The percentiles of the bootstrap repetitions correspond to confidence intervals of the parameters.

\subsection{Application to the Kapuas River}

We apply the method to determine sediment transport in the Kapuas River. The Kapuas is a large sand-bedded river located in West Kalimantan, Indonesia.

\subsubsection{Data collection}

Between December 2013 and July 2014 we collected 276 water samples simultaneously with vessel-mounted ADCP data in the tidally influenced reaches of the Kapuas River. The particle size distribution was measured in-situ with a LISST during one measurement. The majority of samples was collected at the two largest bifurcations of the coastal plain, situated 45 and $90 \mathrm{~km}$ upstream of the river mouth (figure 5.1). At the bifurcations, the Kapuas is 650 and $550 \mathrm{~m}$ wide. During 2014, the river reached a maximum discharge of 10000 $\mathrm{m}^{3} / \mathrm{s}$ during the wet season (chapter 2). The spring tidal range is on average $1.35 \mathrm{~m}$ at the river mouth (chapter 4). At each bifurcation, two boats measured discharge and sediment concentration in parallel during spring tide and neap tide. Both vessels were equipped with a 1.2 MHz RD Instruments ADCP and a GPS. From one vessel, water samples were continuously taken at predefined locations around the bifurcation at four different depths. The second vessel continuously crossed the bifurcation branches for measurements of discharge. Additional samples collected at nearby field sites during the survey are included in the data set. The goal of these measurements is to gain insight 


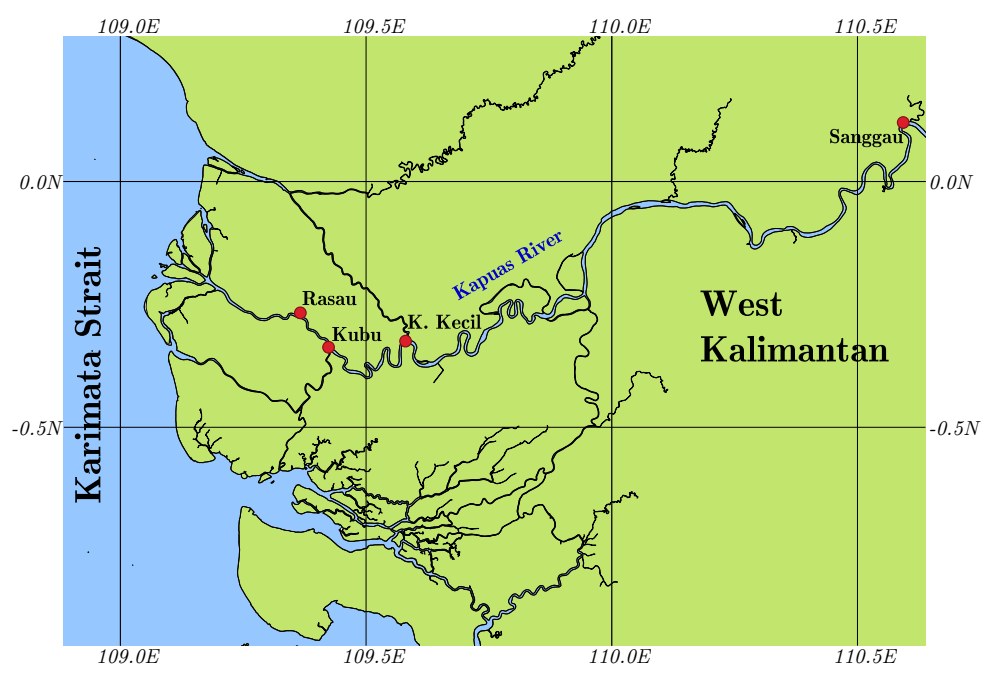

Figure 5.1: Delta of the Kapuas River until Sanggau, the most upstream sampling location, main sampling locations are indicated by red dots. The grid is spaced at $0.5^{\circ} \approx 56 \mathrm{~km}$

into the sedimentary regime of the Kapuas River and the sediment division at the bifurcations, which will be the subject of a follow-up paper.

\subsubsection{Properties of the suspended sediment in the Kapuas}

Figure 5.3a shows the size distribution of the suspended sediment as measured with the LISST at Rasau on the 9th of January 2014, close to neap tide, at an instantaneous discharge of $5500 \mathrm{~m}^{3} / \mathrm{s}$. The mode of the suspended sediment distribution is $85 \mu \mathrm{m}$ and the median $65 \mu \mathrm{m}$. The distribution is skewed towards the silt and clay fractions. The inverse squared backscatter sensitivity $1 / k_{s}^{2}$, computed from the size distribution, is $2029 \mathrm{~kg} / \mathrm{m}^{2}$. According to equation (5.3), the coarsest $3 \%$ of the sediment causes $50 \%$ of the backscatter, and the finest $50 \%$ of the suspended sediment causes less than $1 \%$ of the backscatter. This illustrates that only the coarse fraction of the suspended sediment is sensed by the 1.2 MHz ADCP in the Kapuas. 

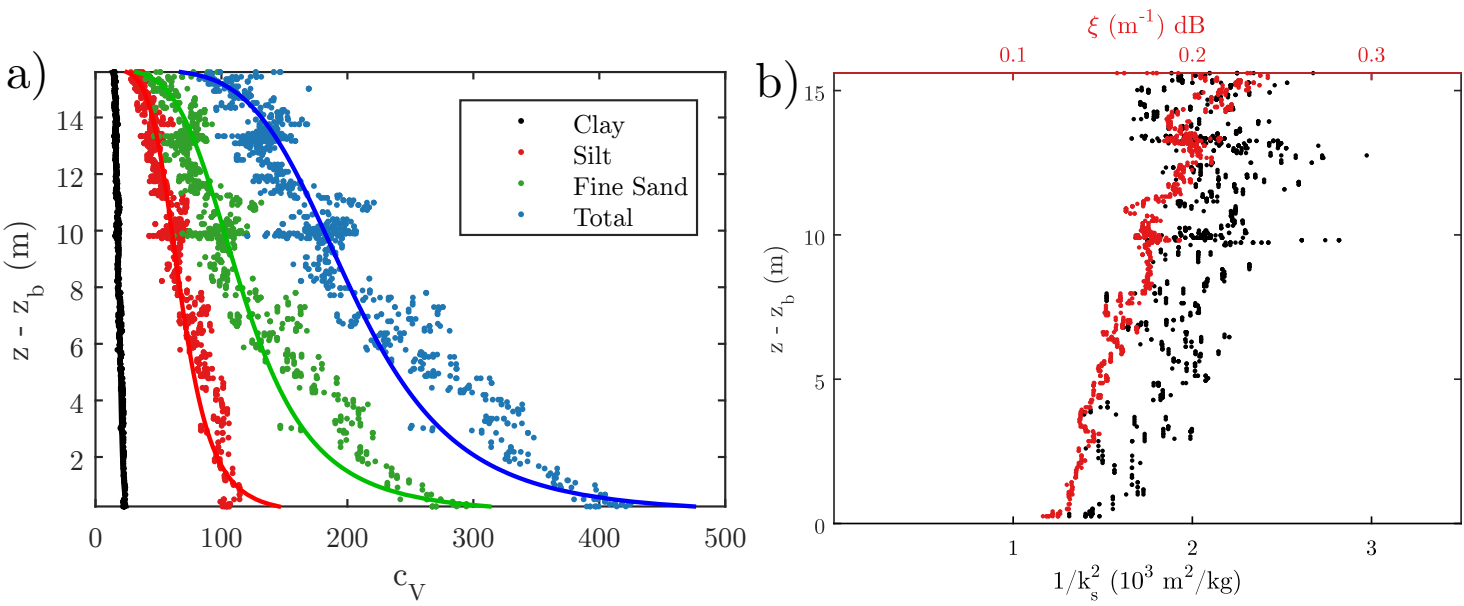

Figure 5.2: Percentage of sand, silt, and clay in suspension along (a) and the backscatter sensitivity, $k_{s}^{2}$, as well as the normalized attenuation coefficient, $\xi$ (b), along the water column at Rasau on 9 January 2014

We estimate the spatial variation of the particle size of the suspended sediment from the grain size of the bed material. A total number of 203 bed samples were collected within a vicinity of $20 \mathrm{~km}$ around the bifurcations (chapter 3 ). The median grain size ( $\left.D_{50}\right)$ of the bed samples is $249 \mu \mathrm{m}$ at the Kecil bifurcation and decreases to $223 \mu \mathrm{m}$, at the Kubu bifurcation (figure 5.3a). The bulk of the bed material has a diameter that is smaller than 350 um (figure 5.3a), but the outer bends consist of coarse material that contains some gravel. Less than $10 \%$ of the bed material samples is composed of coarse sand or gravel. When coarse samples are neglected, the root-mean-square deviation of the $D_{50}$ around the median is $73 \mu \mathrm{m}$. The relative root-mean-square deviation of the inverse backscatter sensitivity, $1 / k_{s}^{2}$, inferred from the bed material, is 1.4. Vertical profiles sampled with the LISST show that that the sediment concentration and particle size decrease between the river bed and the surface according to a Rouse profile (figure 5.2a). The backscatter sensitivity inferred from the particle size distribution increases towards the bottom, and the normalized attenuation constant decreases from the surface to the bottom by 1.5 (figure 5.2b). The spatial variation thus can introduce a noticeable error in the inferred sediment concentration. 

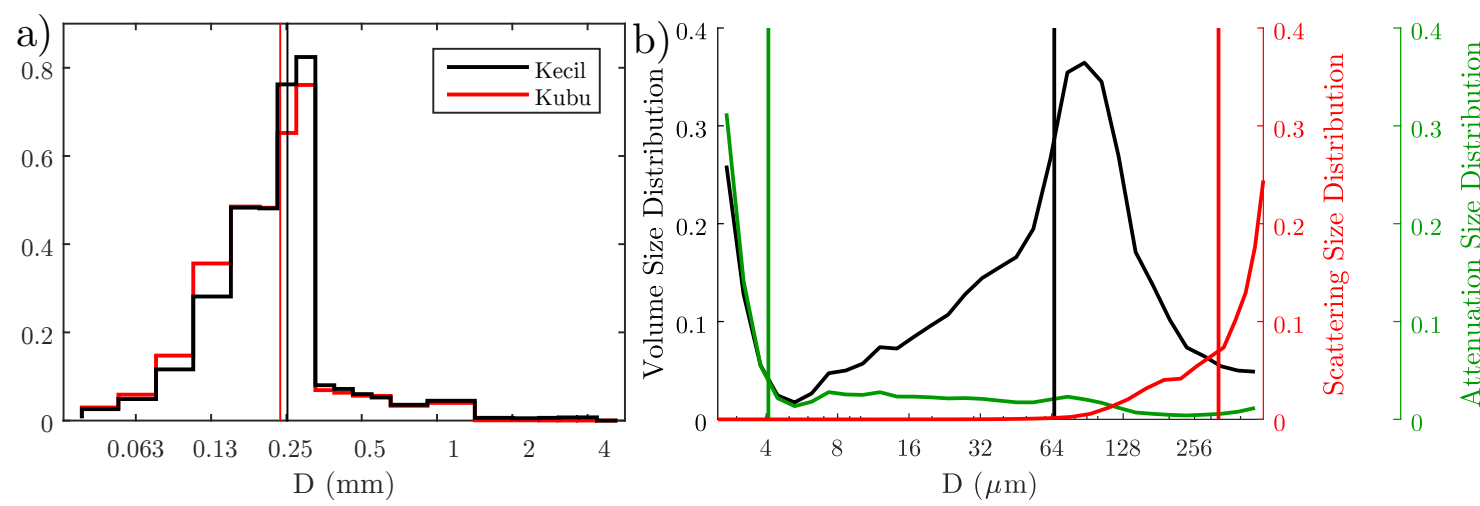

Figure 5.3: Grain size distribution of the bed material in the vicinity of the Kapuas bifurcations (a), vertical lines indicate the medians, and volume size distribution of the suspended load at Rasau (b), as well as the scattering and attenuation weighted distribution. Vertical lines indicate the medians.

The attenuation by water $\alpha_{w}$ during the measurements is $0.26 \mathrm{~m}^{-1}(\mathrm{~dB})$ for the $1.2 \mathrm{MHz}$ transducer at $27^{\circ} \mathrm{C}$. For the suspended sediment, the normalized attenuation coefficient determined from the particle size distribution is $\xi=0.19 \mathrm{~m}^{-1}(\mathrm{~dB})$ and $\xi / k_{s}^{2}=363 \mathrm{~m}^{2} / \mathrm{kg}$. The mass concentration of suspended sediment in the water sampled along with the LISST measurement was $60 \mathrm{~g} / \mathrm{m}^{3}$. The LISST measured a volume concentration of $227 \mu \mathrm{l} / \mathrm{l}$, equivalent to $601 \mathrm{~g} / \mathrm{m}^{3}$, and thus strongly overestimated the suspended sediment concentration. The water remained tanned after filtration due to the presence of dissolved matter. Reduced laser transmission due to dissolved matter is a probable reason for the overestimated sediment concentration by the LISST.

Based on the estimated normalized attenuation coefficient and the sediment concentration determined from the water samples, the two-way attenuation is estimated to be $8 \%$ over a range of $15 \mathrm{~m}$. The sediment concentration for most water samples is below $100 \mathrm{~g} / \mathrm{m}^{3}$, and thus in a similar range as during the LISST measurement (figure 5.6). For typical concentrations observed during our measurements, attenuation would introduce a much smaller error than grain size variation, if it were not corrected for. However, sound was strongly attenuated at one measurement location that corresponds to a recirculation zone where the sediment concentration reached up to $854 \mathrm{~g} / \mathrm{m}^{3}$. Attenuation by mixtures of sand and clay cannot be unambiguously corrected for, as a considerable part of the attenuation is caused by a size fraction that effectively does not scatter (Guerrero et al., 2016). This is also the case in the Kapuas river, as the median of the backscatter weighted particle size distribution is much larger than the median of the attenuation weighted size distribution (figure 5.6). 


\subsubsection{Application of the improved backscatter method}

The mean sediment concentration of the samples in our data set is $72 \mathrm{~g} / \mathrm{m}^{3}$. The sediment concentration of the majority of samples is low, but individual samples reach up to $854 \mathrm{~g} / \mathrm{m}^{3}$. The backscatter intensity increases with the concentration as expected (figure 5.6). We successively fit backscatter models with an increasing number of parameters to the sampled sediment concentration. We compare the model performance by an unpaired test of the rootmean-square-error of the bootstrap repetitions. A plain linear relationship with $1 / k_{s}^{2}$ as the only calibration parameter fits poorly and has a coefficient of determination $\left(\mathrm{R}^{2}\right)$ of merely 0.1 (figure 5.5a). The inclusion of a background concentration $\left(c_{f}\right)$ improves the model performance considerably in all cases $(\mathrm{P}=0)$, but the fit is still not $\operatorname{good}\left(\mathrm{R}^{2}=0.35\right)$. The correction for attenuation by coarse sediment (parameter $\xi$ ) improves the model performance further in $85 \%$ of the cases $(P=0.15)$ and fits the data reasonably well $\left.\mathrm{R}^{2}=0.74\right)$. The correction for the background attenuation $\left(\alpha_{f}\right)$ by fines does not improve the fit further $(\mathrm{P}=0.5)$.
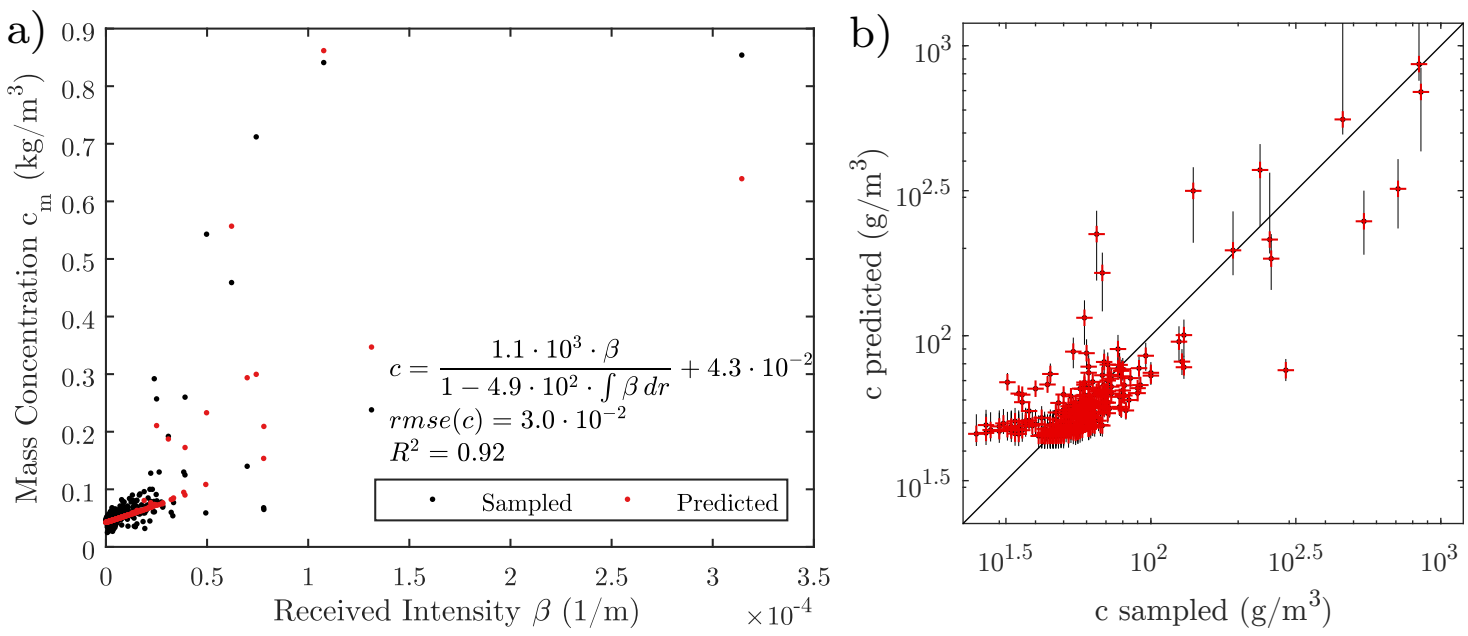

Figure 5.4: Mass concentration $c_{m}$ of suspended sediment versus backscatter intensity, $\beta(\mathrm{a})$, and mass concentration as predicted from backscatter versus mass concentration determined from water samples (b), vertical lines indicate $87 \%$ confidence intervals

We therefore select the model that includes the background concentration and attenuation by coarse sediment, but not the attenuation by fine sediment. For this parametrization, the fitted backscatter sensitivity $\frac{1}{k_{s}^{2}}$ is $1.1 \mathrm{~g} / \mathrm{m}^{2}$. The background concentration of fines $c_{f}$ is $43 \mathrm{~g} / \mathrm{m}^{3}$. The normalized attenuation coefficient $\xi$ is $0.701 / \mathrm{m}\left(\xi / k_{s}^{2}=0.76 \mathrm{~g} / \mathrm{m}^{3}\right)$. All included parameters are signif- 

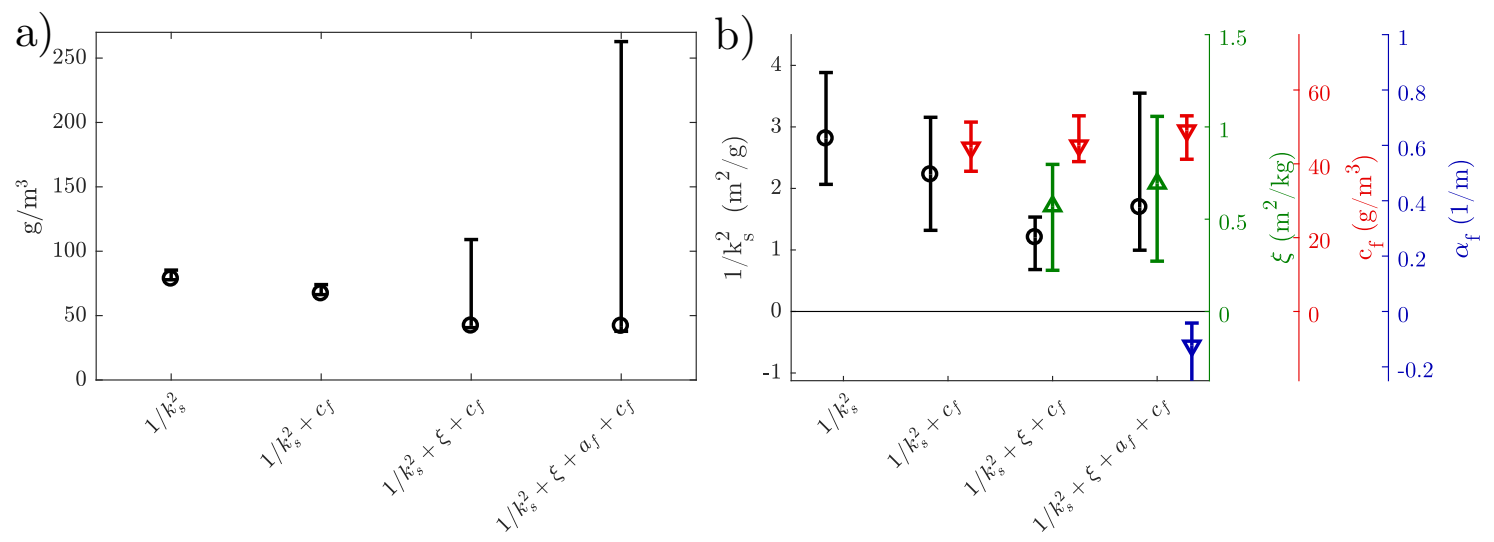

Figure 5.5: Root mean square error with $87 \%$ confidence intervals of the different models (a), based on 1000 bootstrap repetitions, model parameters with $87 \%$ confidence intervals (b)

icantly larger than zero (figure 5.5b). The background attenuation coefficient, if it were included in the model, is insignificantly different from zero and also negative, which is physically implausible.

The ratio of background concentration and mean sample concentration reveals that only $33 \%$ of the total suspended sediment in the samples consists of coarse sediment that is actually sensed by the ADCP. This is expected from the particle sized distribution measured with the LISST. The fitted value of the backscatter sensitivity $\left(1 / k_{s}^{2}\right)$ is lower than that determined with the LISST. This is because the regression model infers only the coarse fraction of the sediment from the backscatter. Indeed, if the background concentration of fines were not included in the model, then the fitted value of the backscatter sensitivity is similar to that determined with the LISST. The fitted values of the normalized attenuation parameter $(\xi)$ is higher than that determined with the LISST, although we expect a lower value so that the correction for attenuation that scales with $\xi / k_{s}^{2}$ remains the same. The single LISST sample may not be representative for the attenuation across the entire set of water samples, which can be expected from the spatial variation of the bed material. Attenuation was low for most samples. The attenuation correction accounts only for $13 \%$ of the sediment concentration. The overall low sediment concentration most likely also explains why the background attenuation by fine sediment $\left(\alpha_{f}\right)$ is low.

The residual of the predicted sediment concentration is more scattered for samples with high concentrations, i.e. the distribution of the residuals is heteroskedastic (figure 5.4b). This skews the distributions of the root-mean- 
square-error towards large values. In particular, the parametrization that includes the correction for attenuation has a poor worst-case performance (figure 5.5a). This is because the attenuation can only be reliably fit when the reference data includes samples with high concentrations. This is not necessarily the case for all bootstrap repetitions, as there are only few samples with high concentrations in our data set. The heteroskedasticity also causes the distribution of the backscatter sensitivity $\left(1 / k_{s}^{2}\right)$ to be skewed towards large values (figure 5.5b).

\subsubsection{Validation}

We validate the splitting of the suspended sediment into a coarse fraction and a fine fraction by comparing the time series of backscatter and suspended sediment concentration over a tidal cycle. As the imperceptible fraction is fine, it has to be more homogeneously distributed in the water and to settle less rapidly than the coarse sensed fraction. Figure 5.6 shows the time-series of the cross-section averaged backscatter and of the sediment concentration of the water samples for one complete tidal cycle on 27 March 2014. The backscatter intensity responds rapidly to the change in velocity without a noticeable lag, as expected from the high settling velocity of the coarse fraction that causes most of the scattering. The backscatter intensity decreases to zero around slack water. The sediment concentration in the water samples likewise responds to changes of the flow velocity, which confirms the presence of coarse suspended material. In contrast to the backscatter, the concentration of sediment in the water samples does not drop to zero but remains around $45 \mathrm{~g} / \mathrm{m}^{3}$ during slack water. This confirms the presence of fine sediment, which does not settle rapidly so that it is transported as washload. The presence of washload is also indicated by the turbidity of the near-surface water that does not clear during slack water, as well as the absence of mud drapes in the bed material. Consequently, only the coarse sand fraction of the suspended sediment can be inferred from the ADCPbackscatter.

\subsection{Discussion}

It is difficult to measure the suspended sediment concentration with monofrequency ADCPs, when the particle size distribution is wide, as one cannot differentiate between changes of the particle size distribution and sediment concentration. Acoustic backscatter is typically dominated by sand (Guerrero et al., 2013, 2017) so that surrogate measurements only sense the variation 


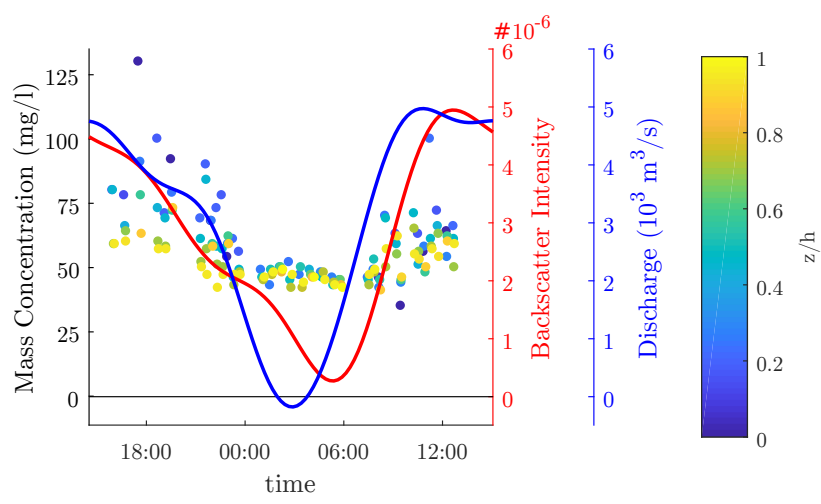

Figure 5.6: Variation of sediment concentration and received intensity over the diurnal cycle on 27th March 2014 at the Kubu bifurcation

of the coarse fraction (Bunt et al., 1999). Both the sediment concentration as well as the size distribution usually depend on the flow velocity (McLean, 1991; van Rijn, 1984b) so that the size distribution of suspended sediment in rivers can vary considerably over the tidal cycle.

The background concentrations of fine sediment found in the Kapuas are usual for estuaries (Fugate and Friedrichs, 2002; Tattersall et al., 2003) and rivers that carry fine sediment (Wolanski et al., 1996). In main and side channels of braided rivers, the grain size distributions can even be bimodal (Guerrero et al., 2016). The inclusion of a background concentration of fine material improves the fit considerably and significantly in our case. This approach also reveals which part of the sediment concentration is actually sensed by the ADCP, and which is not. The parameter $c_{f}$ represents the average background concentration of fines in the calibration data, and any variation of the washload during subsequent ADCP measurements, where the calibration is used, is not sensed. This does not affect the inferred concentration of the coarse sediment, as long as the attenuation by fines is not large.

The acoustic backscatter in the Raleigh regime is more sensitive to changes of the particle size than to changes of the concentration, as it increases cubically with the size but only linearly with the concentration of the suspended sediment. Small changes in the particle size can therefore introduce large errors in the inferred sediment concentration (Venditti et al., 2016). When the size of the suspended sediment is correlated with the concentration, then the sediment concentration can be inferred from the backscatter by a power law:

$$
c=\tilde{a} \beta^{\tilde{b}} .
$$

Buschman (2011) and Hoitink (2004) found similar powers for the Berau $(\tilde{b}=0.41)$ and Bay of Banten $(\tilde{b}=0.4)$. The empirical relationship (5.6) for the 
Mahakam (Sassi et al., 2012) can be recast into a power law as well for a reference point close to the transducer:

$$
c_{0} \approx \frac{1}{a} \beta_{0}^{(1-b)} .
$$

For the Mahakam, Sassi et al. (2012) found $a=1$ and $b=0.45$, which results in $\tilde{b} \approx 1-b=0.55$. The power law does not correct for attenuation between the transducer and the reference point, because then $1-b$ is larger than unity. We argue that equation (5.6) corrects for the change of the particle size over the tidal cycle. Similar powers near 0.5, obtained for different field sites (Hoitink, 2004; Buschman, 2011; Sassi et al., 2012), suggests some generality of the approach. However, the change of the particle size distribution in the Kapuas over the tidal cycle is too strong to be captured by a simple power law.

\subsection{Conclusion}

We propose a method to reduce the ambiguity of suspended sediment concentrations inferred from mono-frequency backscatter, which is often present when the size distribution of the suspended sediment is broad. The method separates the fraction of coarse sediment that is sensed by the ADCP from the fraction of fine sediment that is not sensed by the ADCP, by means of a linear regression. The method is straight forward to apply and does not require explicit knowledge of the particle size distribution. Application to a dataset collected in the Kapuas River shows it is possible to monitor suspended sand transport with an off-the-shelf ADCP in a tidally influenced environment where the spatiotemporal variations of wash load and suspended sand are poorly correlated. 


\section{Flow and sediment division at two asymmetric bifurcations of a tidally influenced river delta: implications for channel stability}

The division of sediment at large river bifurcations results from the complex 1 interaction between the three-dimensional flow, transport of mixed sediments and channel bed morphology. Especially in near-pristine regions, only few field measurements are available, which is why the details of these interactions remain poorly understood. We present measurements of the flow and sediment division at two tide-influenced asymmetric bifurcations of the Kapuas River, a large sand-bedded river in Kalimantan, Indonesia. At both of these bifurcations, small channels branch off from the side of the main river. Flow velocities are measured with a boat mounted acoustic velocity profiler. Sediment concentrations are determined from water samples and inferred from acoustic backscatter. We find that the bulk of the sediment is transported in suspension. The suspended material reaches from fine sand, which rapidly responds to the temporal variation of the flow velocity over the tidal cycle, to silt and clay, which mostly remain suspended during slack water. The side channel of the first bifurcation branches off in an outer bend so that sand primarily bypasses the side channel. The side channel at the other bifurcation branches off in a straight reach and receives proportionally a large amount of sediment. Based on the field measurements, we explore the reasons why both bifurcations have been stable at least since the first accurate mapping in the late $19^{\text {th }}$ century.

This chapter is based on: Kästner, K., and A. J. F. Hoitink, Flow and sediment division at two asymmetric bifurcations of a tidally influenced river delta: implications for channel stability, Submitted to Journal of Geophysical Research: Earth Surface, 2019a 


\subsection{Introduction}

River bifurcations determine the path along which water and sediment are transported towards the sea (Syvitski et al., 2005a). The division of water and sediment can change over time, existing delta branches can be abandoned, and new branches can be formed. Such rearrangements can have a devastating impact on human settlements (Goodfriend and Stanley, 1999; Slingerland and Smith, 2004; Anthony et al., 2015). Therefore, delta networks are often maintained in a fixed state by engineering works and continuous maintenance (Syvitski and Saito, 2007). With increasing population density and economic growth, the last pristine delta networks are about to be developed into managed waterways, and only few bifurcations will retain their natural dynamics. At the same time, a trend is emerging to revitalize natural processes in managed deltas (Costanza et al., 2006; Rijke et al., 2012). Such efforts call for a thorough understanding of processes governing the hydro- and morphodynamics of the remaining near-pristine channel systems on earth.

A common configuration of a river bifurcations is an approaching channel that splits symmetrically around a delta-shaped island. This is typical for bifurcations around mouth bars (Edmonds and Slingerland, 2007) and braid bars (Zolezzi et al., 2009). However, many bifurcations evolve over time such that one of the branches becomes larger and the other one smaller because asymmetrical bifurcations are intrinsically more stable (Axelsson, 1967; Edmonds and Slingerland, 2008; Zolezzi et al., 2009). During this process, the banks of the larger branch align with that of the approaching channel, so that the angle with the smaller branch increases. Channels that form by avulsion branch off to the side and are therefore asymmetric from the moment of their formation (Hood, 2010).

The geometry of a bifurcation strongly influences the division of sediment over the downstream branches, because the secondary flow and the bed topography redirect the sediment. This is different from the division of water, which is primarily determined by the size of the bifurcation branches and downstream boundary conditions (Edmonds, 2012). Channels that branch off laterally receive a proportionally larger fraction of sediment than water, because the secondary flow steers the near-bed flow with high sediment concentration into the side branch (Bulle, 1926; Kawai et al., 1993; Neary et al., 1999; Aǵaçcioǵlu and Önen, 2005; Emiroglu et al., 2011; van der Mark and Mosselman, 2013; Michelazzo et al., 2015; Herrero et al., 2015; Gaweesh and Meselhe, 2016; Dutta et al., 2017). Bifurcations, where the sediment division is primarily governed by secondary flow, are therefore prone to be ephemeral, 
as the side branches fill in over time. To keep a side-branch open, proportionally less sediment than water has to be diverted into the smaller branch, as the transport capacity decreases non-linearly with the discharge (Wang et al., 1995; Salter et al., 2018). Here, we focus on side branches in natural deltas that defy, or at least defer, the fate of silting up and becoming abandoned by the river flow. We aim to understand the reasons for strongly asymmetrical channel bifurcations in lowland rivers to remain stable.

The stability of strongly asymmetric bifurcations in lowland rivers may partly be explained from bedload transport being diverted away from a smaller and shallower branch, when the bed slopes of the approaching channel slopes down towards the larger and deeper branch (Edmonds and Slingerland, 2008; Bolla Pittaluga et al., 2003). Yet even if such a slope is absent, sediment can bypass a side branch when it connects to the main channel in an outer bend, as the bulk of sediment is transported along the inner bend (Kleinhans et al., 2008; van Denderen et al., 2018). In addition, a relatively large contribution of suspended load in the total sediment transport may play an important role. Suspended load can bypass a side branch when the bed level steps up towards the branch so that especially water that flows near the surface is being diverted, where the sediment concentration is low (Slingerland and Smith, 1998). The division of sediment depends on the river stage (Edmonds, 2012) and can vary during the passage of a flood wave (Frings and Kleinhans, 2008a). Finally, tides may play a role in keeping side channels open, by influencing both the channel geometry and the division of river discharge (Buschman et al., 2013; Sassi et al., 2011a, 2013).

This contribution aims to establish the physical mechanisms controlling the sediment division of fine sand at two pristine, strongly asymmetrical channel junctions in a large suspended load dominated sand bed river. We focus on two tidally influenced bifurcations of the Kapuas River, West Kalimantan, Indonesia. We analyse the bed morphology of the bifurcations, examine the physical processes governing the discharge and the sediment division over a tidal cycle, and discuss the implications for channel stability. Section 6.2 introduces the field sites. In Section 6.3, field measurement procedures are described. Section 6.4 presents the results of the measurements and in Section 6.5 the main findings and their implications are discussed.

\subsection{Field Site}

The Kapuas River is a meandering river that has not yet been restrained by dams, dykes or groins. It flows through West Kalimantan, Indonesia, and dis- 
charges into the Karimata Strait. The river has a catchment area of $99000 \mathrm{~km}^{2}$ (Hidayat et al., 2017) and its discharge is governed by the monsoons. Between December 2013 to April 2015, we monitored discharge at Sanggau, $285 \mathrm{~km}$ upstream from the sea (chapter 2). During this period, the discharge ranged between $1000 \mathrm{~m}^{3} / \mathrm{s}$ in the dry season and $10000 \mathrm{~m}^{3} / \mathrm{s}$ in the wet season. There are no major confluences downstream of Sanggau. The tide is mainly diurnal (chapter 4). Spring tides have one cycle per day and range on average $1.4 \mathrm{~m}$ at the river mouth, whereas neap tides have two cycles per day and range on average $35 \mathrm{~cm}$. During the dry season, tidal waves intrude beyond the city of Sanggau.

For the last $200 \mathrm{~km}$, the Kapuas flows through its alluvial plain. Along the plain three small distributaries branch off, which are called Mendawat, the Kapuas Kecil and the Kubu (Figure 6.1). The cross-section geometry of the main river, the Kapuas Besar, does not abruptly change at either of the bifurcations, but rather gradually narrows and deepens along the alluvial plain (chapter 3). The Kapuas Besar terminates into a small radial delta about $17 \mathrm{~km}$ upstream from the sea, which has five outlets. The Mendawat and Kubu branch off to the South and are connected to a network of tidal creeks. The Kapuas Kecil branches off to the North and is joined by a confluence $21 \mathrm{~km}$ upstream from the sea. All distributaries remain nearly constant in width before terminating in a funnel-shaped section. Salinity only reaches the Kubu bifurcation during extreme low flow and does not reach the Kapuas Kecil bifurcation.

The Kapuas Kecil bifurcation is located $85 \mathrm{~km}$ upstream from the mouth of the Kapuas Besar, and $74 \mathrm{~km}$ upstream of the mouth of the Kapuas Kecil. The Kapuas Kecil branches off in an outer bend of a meander loop that is $7 \mathrm{~km}$ long. The bend straightens close before the diversion and tightens again just downstream of the diversion. In the vicinity of the bifurcation, the Kapuas Besar is $525 \mathrm{~m}$ wide and $16 \mathrm{~m}$ deep when averaged over the entire reach. The Kapuas Kecil is $220 \mathrm{~m}$ wide and $12 \mathrm{~m}$ deep. The Kapuas Kecil curves away smoothly so that there are no sharp corners at the diversion. The Kapuas Kecil branches off initially at $125^{\circ}$ but continues to turn around the hook to $160^{\circ}$. The inlet is approximately $500 \mathrm{~m}$ wide, more than twice as wide as the channel further downstream.

The Kubu Bifurcation is located $47 \mathrm{~km}$ upstream from the mouth of the Kapuas Besar, and the river bifurcates once more before it arrives at the coast. One branch flows into the sea $57 \mathrm{~km}$ from the bifurcation and the other branch flows into the Selat Panjang estuary, $40 \mathrm{~km}$ from the bifurcation. In the vicinity of the bifurcation, the Kapuas Besar is $420 \mathrm{~m}$ wide and $20.5 \mathrm{~m}$ deep and the Kubu channel is $190 \mathrm{~m}$ wide and $7.5 \mathrm{~m}$ deep. The approaching channel is 


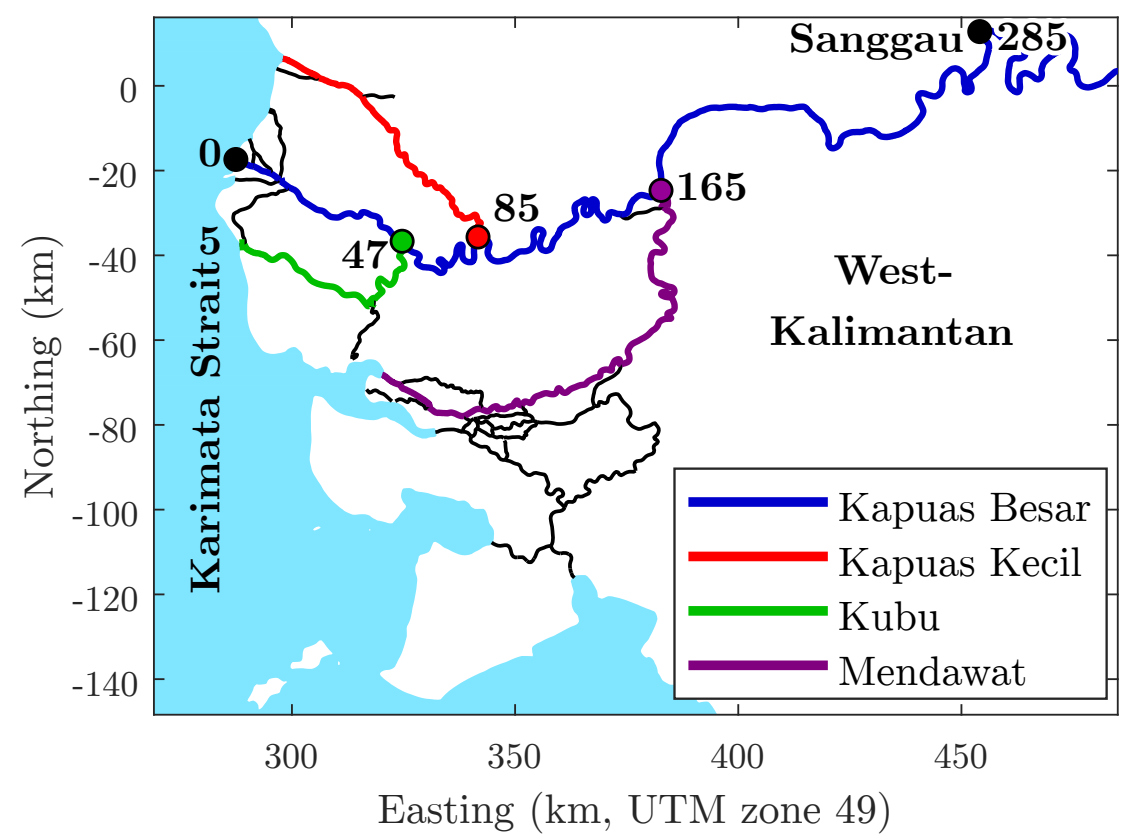

Figure 6.1: Distributary channel network of the Kapuas river (UTM zone 49), markers give distances in $\mathrm{km}$ with respect to the mouth of the Kapuas Besar.

straight for the last $3 \mathrm{~km}$ upstream of the bifurcation and the Kubu channel turns away sharply at an angle of $160^{\circ}$. The entrance of the Kubu channel is $600 \mathrm{~m}$ wide, and thus more than three times as wide as the channel further downstream.

\subsection{Methods}

We measured the flow velocity and sediment concentration from moving vessels at both bifurcations during a spring tidal cycle. The measurements lasted for $25 \mathrm{~h}$ during spring tides to cover the full tidal cycle. We measured with two vessels in parallel. Both vessels were equipped with a $1.2 \mathrm{MHz}$ vertical acoustic velocity profiler (VADCP) and a GPS antenna directly mounted above the VADCP. One vessel circled the bifurcation and crossed the approaching channel as well as the two downstream branches in succession. The second vessel also circled the bifurcation but took water samples on its way. We collected water samples at three locations along each of the two main channel transects and two water samples along the side channel transects. The samples taken in the main channel consist of batches of four point-samples along the vertical and the samples along the side branches of two point-samples along the vertical. Water was sampled with a 2.51 Niskin bottle. A $200 \mathrm{ml}$ volume of the 
sample was split off in a mixed bucket for further processing in the lab. The water samples were vacuum filtered on micropore membranes and dried. The mass concentration was determined as the ratio of the mass of the sediment retained on the filters and the total mass of the water sample.

The bathymetry and bed material was surveyed separately, as reported in (chapter 3). Water levels were gauged in the main channel $10 \mathrm{~km}$ up and $4.5 \mathrm{~km}$ downstream of the Kapuas Kecil bifurcation, as well as $11 \mathrm{~km}$ downstream and $12 \mathrm{~km}$ upstream of the Kubu bifurcation (chapter 4). The acoustic backscatter was calibrated to infer the suspended sand concentration as described in (chapter 5. Suspended sediment was found to consist of silt and clay in addition to sand, but these fractions do hardly scatter the sound transmitted by the ADCP and are therefore not represented here. We consider the morphodynamics to be primarily controlled by the division of sand over the distributaries, as the riverbed consists of sand and the fine fractions remain mostly suspended throughout the tidal cycle.

\subsection{Results}

\subsubsection{Bed level at the Kapuas Kecil bifurcation}

The bed at the Kapuas Kecil bifurcation is shaped by the helical flow along the meander bend, which created a $30 \mathrm{~m}$ deep thalweg in the outer bend and a point bar in the inner bend (Figure 6.2a). The side channel perturbs the longitudinal bed profile at the bifurcation so that the thalweg to locally rise up to a depth of $16 \mathrm{~m}$. Downstream of the bifurcations, the thalweg plunges into a scour hole that exceeds $40 \mathrm{~m}$ in depth. The scour hole stretches into the Kapuas Kecil. The spit that separates the Kapuas Kecil from the Kapuas Besar protrudes as a submerged levee into the side channel.

The track navigated with the boat during the measurements is shown in Figure 6.2b. The upstream channel consists of a u-shaped section that (Figure $6.4 \mathrm{a}$ ) extends over the outer $4 / 5^{\text {th }}$ of the total width. It reaches a maximum depth of $25 \mathrm{~m}$ about $1 / 5^{\text {th }}$ from the outer bank. The sides of the u-shaped channel are convex so that there is a sharp transition to the bar. The bar extends over the innermost $1 / 5^{\text {th }}$ of the total width and is less than $3 \mathrm{~m}$ deep. There is no submerged mid-channel bar that anticipates the bifurcation. The cross-section at the downstream transect (Figure 6.4b) is slightly larger than at the transect of the approaching channel. It has a $\nu$-shaped channel that reaches a maximum depth of $40 \mathrm{~m}$ in the outer bend. The bed in the inner bend is convex so that there is a gradual transition to the bar. The bar stretches 
a)

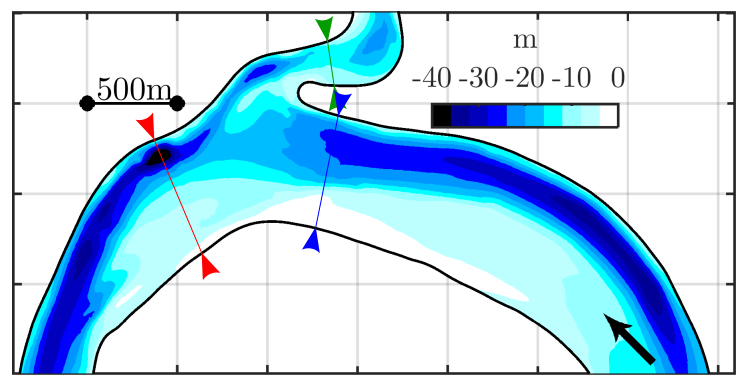

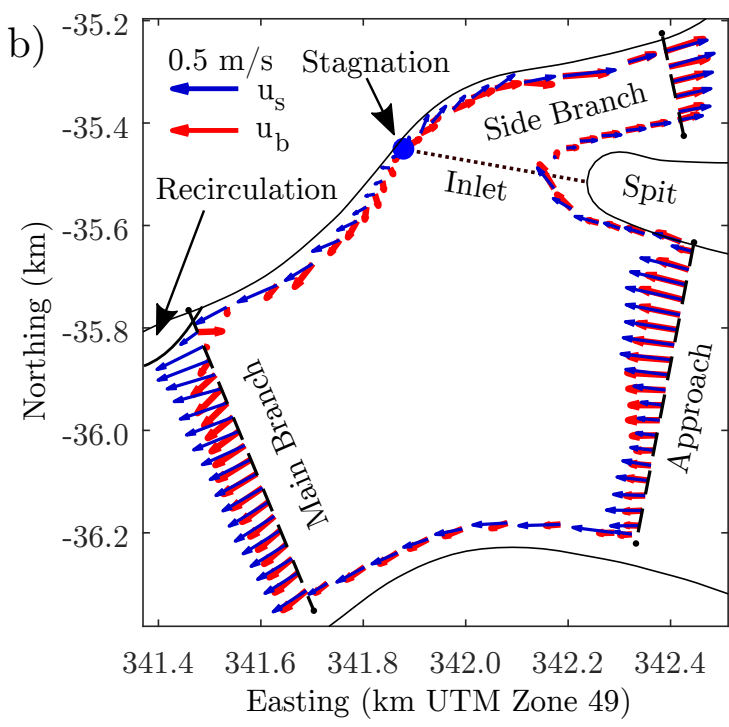

Figure 6.2: Bed level at the Kapuas Kecil bifurcation (a); Tidally averaged near surface $\left(u_{s}\right)$ and near-bed $\left(u_{b}\right)$ flow velocity along the boat track of the Kecil bifurcation on 22 April 2014 (b)

up to the centre of the channel and is $7.5 \mathrm{~m}$ deep. The transect across the side branch (Figure 6.4c) consists of a u-shaped cross-section that reaches a maximum depth of $15 \mathrm{~m}$ on the left side.

Near the bifurcation, the Kapuas Kecil has a much larger cross-section area than further downstream so the cross-section area gradually decreases into the downstream direction. At the downstream transect, the cross-section area of the Kapuas Kecil has reached its reach-averaged size.

\section{Discharge division}

The Kapuas Kecil survey spans the diurnal tidal cycle of 22 April 2014. For this day the tidal range is $1.19 \mathrm{~m}$ at the river mouth. The measured tide-averaged discharge from upstream is $3850 \mathrm{~m}^{3} / \mathrm{s} .17 \%$ of the discharge flows into the Kapuas Kecil and 83\% continues along the Kapuas Besar. The cross-sectionally averaged velocity is highest in the upstream transect (Figure 6.3a), slightly lower than that in the downstream transect and lowest in the shallow side branch. The tide is strongly asymmetric so that velocity remains nearly constant during ebb tide. The half-range of the tidal discharge was $2570 \mathrm{~m}^{3} / \mathrm{s}$. The flow, therefore, did not reverse in the main branch that day and just reversed shortly in the side branch. The highest water level (HW) and minimum velocity are associated with peak flood. 

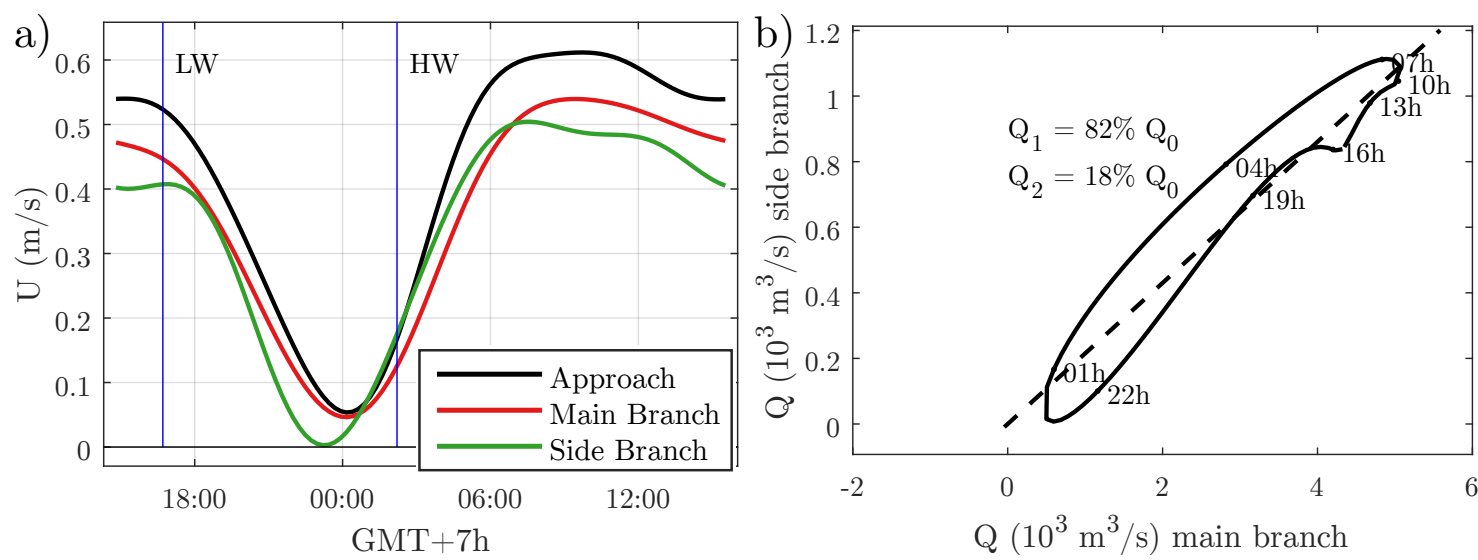

Figure 6.3: Cross-sectionally averaged flow velocity (a) and discharge division (b) at the Kapuas Kecil bifurcation on 22 April 2014. The vertical line indicates the time of high water.

The minimum velocity occurs in the main channel $2 \mathrm{~h}$ before HW and thus leads the water level variation in the diurnal cycle by $29^{\circ}$. The minimum velocity in the side branch occurs 3:15 h before HW, which corresponds to $47^{\circ}$. Flow variation in the K. Kecil branch is thus ahead of that in the K. Besar. This is remarkable, as the tide was expected to take longer to propagate up the side channel, which has a lower wave celerity than due to its shallower depth (chapter 3). This might be related to phase differences at the channel mouths.

\section{Flow}

The spatial pattern of the flow velocity did not vary considerably over the tidal cycle. Since the flow did not reverse, we present here only the peak flow during ebb tide. In the upstream transect (Figure 6.4a), the velocity in the outer bend is more than twice as large as in the inner bend. The maximum of the velocity is located $1 / 5^{\text {th }}$ of the width away from the bank in the outer bend. There is a rapid transition from low to high velocity near the channel centre. The part with the high velocity only comprises half the cross-section. From the centre of the u-shaped bifurcation, the flow diverges, anticipating the bifurcation further downstream (Figures 6.4 and 6.5). There is no secondary flow cell, which may be due to the reduction of curvature of the bend just upstream of the diversion. The velocity increases towards the outer bend both near the bed and near the surface (Figures 6.5 and $6.2 \mathrm{~b}$ ). The velocity profile in the left half of the transect across the bifurcation strongly deviates from a logarithmic profile, as the velocity in the lower half of the water column is higher than in the upper half. The strength of the tidal flow relative to the 
river flow is largest at the bar, where the flow shortly reverses (Figure 6.6). Across the downstream transect in the main channel (Figure 6.4b), the flow velocity increases from the inner bend towards the outer bend and is fairly evenly distributed. There is a pronounced secondary circulation cell in the outer bend. Close to the bank in the outer bend, the streamwise velocity is weak but the downflow is very strong. At the bank near the outer bank, the flow separates in the vicinity of the transect (Figure 6.5b). Near the bed, the flow reverses further upstream than near the surface so that near the shear layer the near-surface flow is directed into the opposite direction with respect to the near-bottom flow. The flow separates only locally over the scour hole downstream of the diversion. The separation thus does not reach into the side branch (Figure 6.2b).

The tidal flow is stronger in the inner bend than in the outer bend so that the flow shortly reverses during flood tide in the inner bend (Figure 6.6b). The near-surface flow remains directed along the main channel, while the flow near the bed is deflected to the side branch. The flow enters the diversion at a low velocity and accelerates towards the transect in the downstream branch. Towards the side-branch, the flow decelerates (Figures 6.4c and 6.5c).
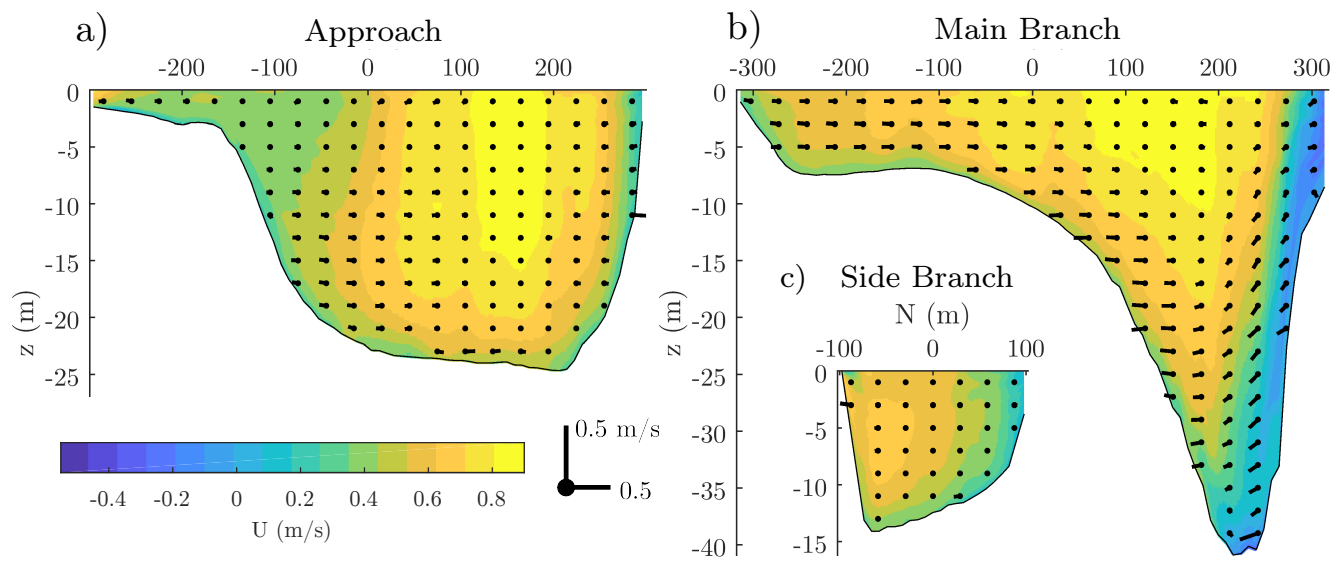

Figure 6.4: Flow velocity in the branches of the Kecil bifurcation during peak ebb tide on 22 April 2014. Elevation is 10 times exaggerated

\section{Pathways of suspended sand}

Across the transect of the approaching channel (Figure 6.7a), the concentration of suspended sand increases towards the bed. The concentration is high in the inner $4 / 5^{\text {th }}$ of the bend and very low near the outer bank, from where the 

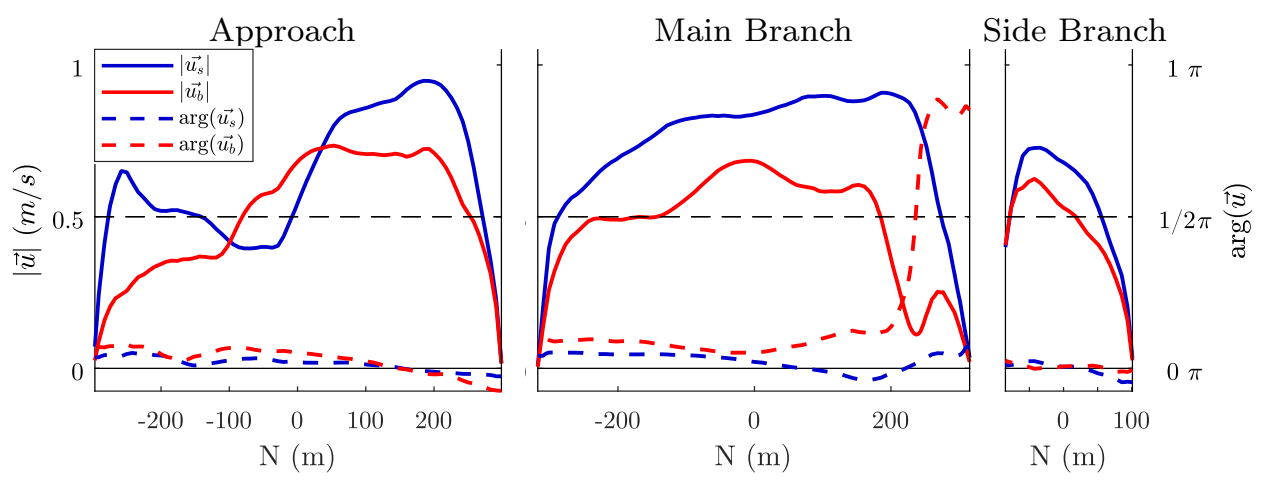

Figure 6.5: Magnitude (solid) and angle (dashed) of the near surface (blue) and near-bed (red) flow along the boat track at the Kapuas Kecil bifurcation during peak ebb tide on 22 April 2014; the flow angle is measured with respect to the lateral direction along the cross-sections as shown in Figure 6.2a.

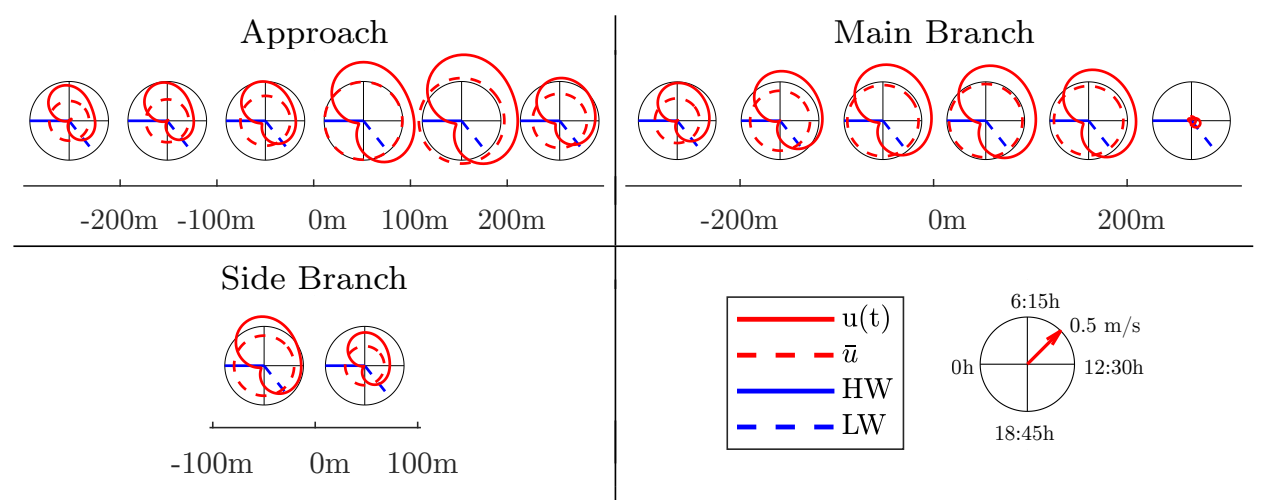

Figure 6.6: Flow velocity over the full tidal cycle of $25 \mathrm{~h}$ in intervals of $100 \mathrm{~m}$ for each crossing (bold red loops). Mean flow velocity is indicated by the dashed red circle. Time passes clockwise. Time of high water is indicated by the bold hand and time of low water by the dashed hand. Start time is shifted so that high water occurs at $0 \mathrm{~h}$.

flow is diverted further downstream. Near the centre of the u-shaped channel, moderately high concentrations reach up into higher parts of the water column. Therefore, the depth-integrated sand transport reaches a maximum near the centre of the u-shaped channel and rapidly drops to either side (Figure 6.8a). In the downstream transect (Figure 6.7b), the concentration of 
suspended sand is higher in the inner bend than in the outer bend, as in the approaching channel. The depth-integrated sand transport is slightly higher in the inner bend than in the approaching channel, which is due to the larger depth (Figure 6.8a). High concentrations reach up high into the water column about $1 / 3$ of the width from the outer bank. Here, the concentration reaches a maximum in the upper part of the channel centre and decreases towards the bed so that the vertical profile of the suspended sand does not resemble a Rouse profile. This is because the secondary flow advects sediment-rich water in the upper layer from the inner bend towards the outer bend and, vice versa, sediment-poor water in the lower layer from the outer towards the inner bend. The suspended sand concentration is very low in the scour hole and slightly decreases in the recirculation zone near the right bank. In the side branch, the suspended sand concentration is very low (Figure 6.7c), which agrees with the low concentration in the outer bend of the approaching channel, from where the flow is diverted. The bulk of the suspended sand thus remains in the main channel, and only 7\% enters the Kapuas Kecil, less than half of the fraction of the diverted water (Figure 6.8b).
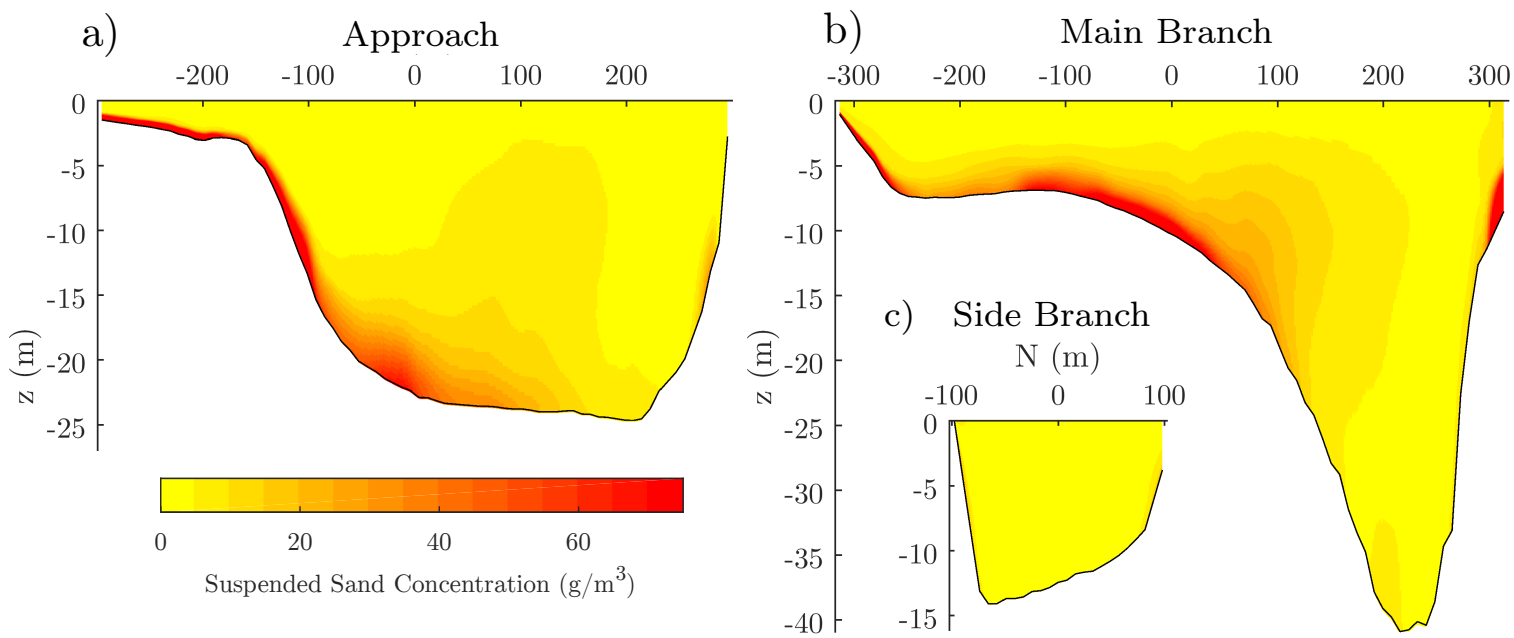

Figure 6.7: Suspended sand concentration in the branches of the Kapuas Kecil bifurcation during peak ebb flow on 22 April 2014

\subsubsection{Kubu bifurcation}

The bed at the Kubu bifurcation slopes down across the approaching channel towards the left bank, from where the Kubu channel branches off (Figure 6.9). This is accentuated by a trench near the left bank that gradually deepens towards the bifurcation, and drops into a scour hole at the downstream end of 

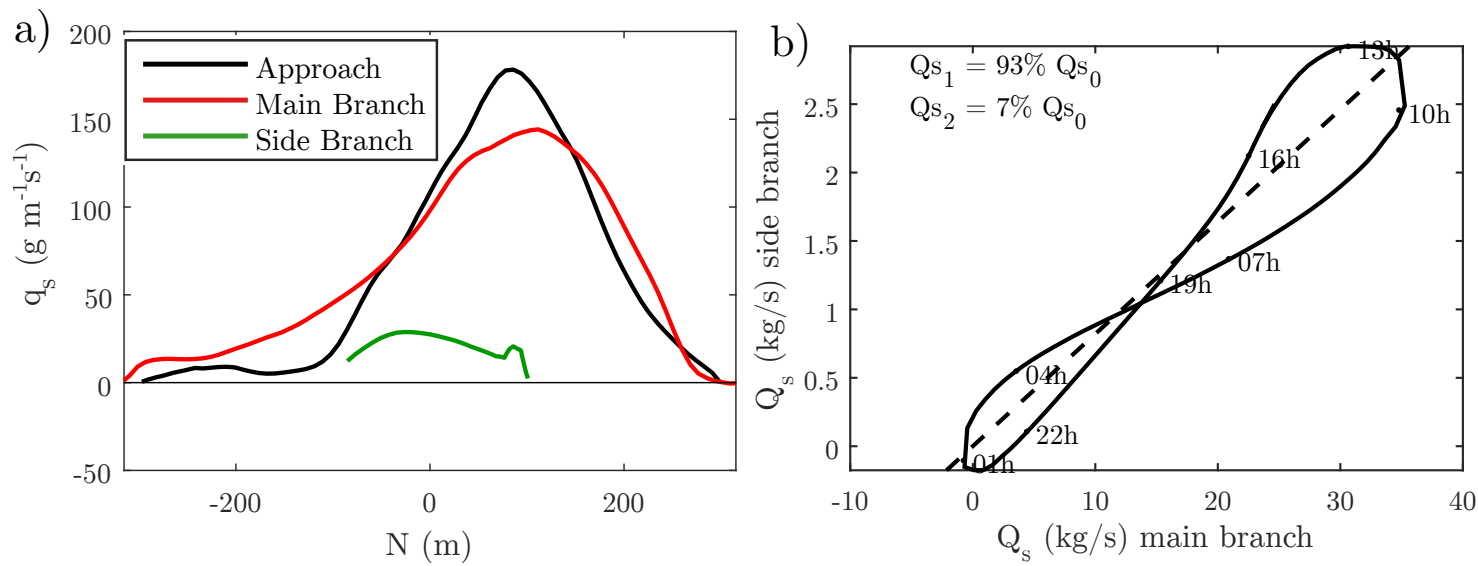

Figure 6.8: Depth-integrated suspended sand transport across the approaching channel and branches of the Kapuas Kecil bifurcation on 22 April 2014 during peak ebb tide (a), corresponding total suspended sand transport over the tidal cycle (b)

the diversion, where it is $35 \mathrm{~m}$ deep. Downstream of the diversion, the main branch, the Kapuas Besar, turns right and locally narrows. The scour hole extends into the narrow section where it reaches its maximum depth. The scour hole also reaches into the Kubu branch at the downstream end of the diversion, while at the upstream end the left bank of the main channel continues as a submerged levee. At the bifurcation, the depth is slightly lower than in the approaching channel. At the upstream transect, the trench near the left bank is $21 \mathrm{~m}$ deep (Figure 6.11a). There is also a smaller trench at the opposite bank that is $14 \mathrm{~m}$ deep so that the central part of the cross-section is slightly elevated. The downstream transect crosses the river before the diversion is complete and thus covers both the main branch and the initial part of the Kubu branch (Figure 6.11b). The geometry of the downstream transect is very similar to that of the upstream transect. The trench on the left side is $19 \mathrm{~m}$ deep and remains separated from the Kecil branch by the submerged levee. The inlet to the side branch is scoured to $29 \mathrm{~m}$ at the downstream transect. At the transect of the Kecil branch (Figure 6.11c), the cross-section has reached its reach-average depth of $7.5 \mathrm{~m}$. Remarkably, the inlet area of the side branch is nine times as large as the reach-average cross-section of the Kubu channel further downstream.

\section{Discharge division}

The Kubu measurement spans the tidal cycle at spring tide on 27 March 2014. For this day, the tidal range of this day is $1.03 \mathrm{~m}$ at the river mouth. The mea- 

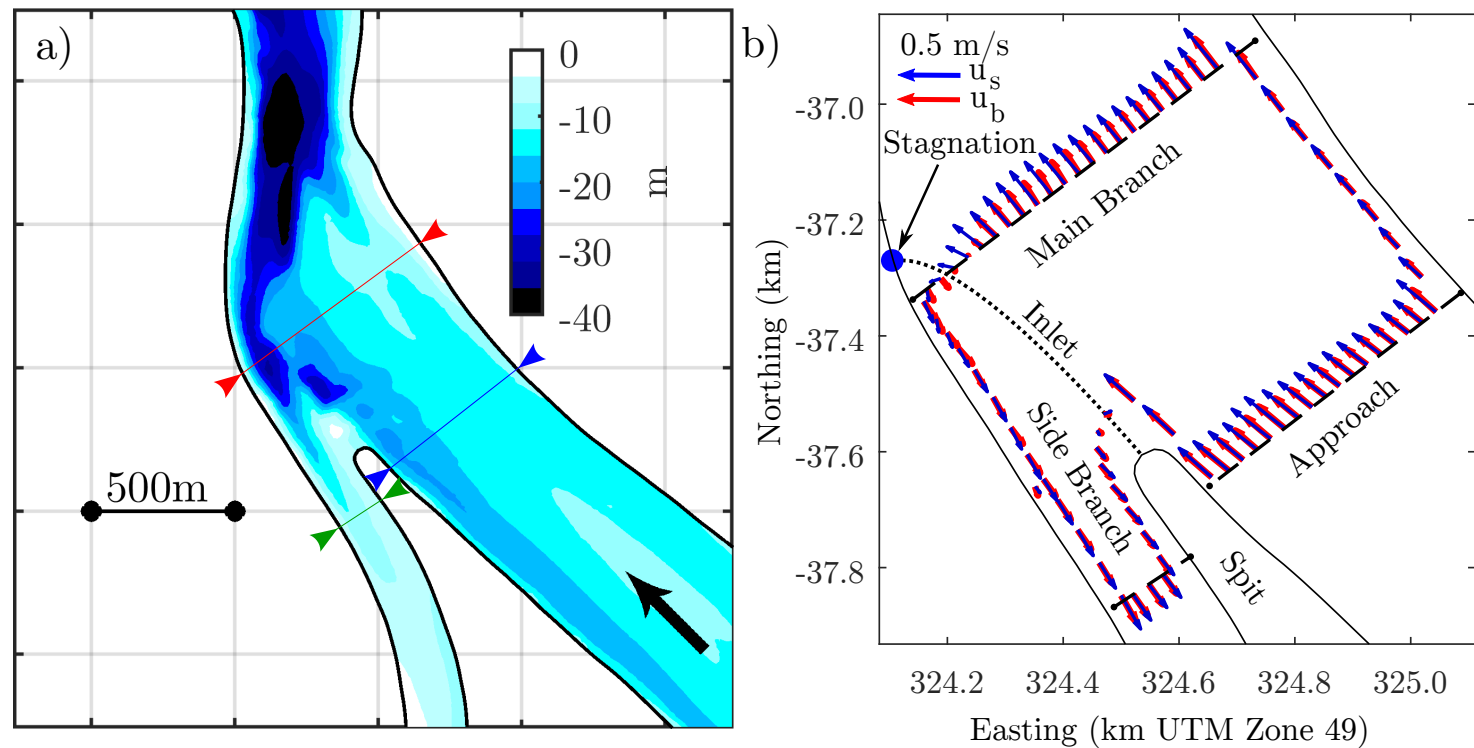

Figure 6.9: Bed level at the Kubu bifurcation (a); Tidally averaged near surface $\left(u_{s}\right)$ and near-bed $\left(u_{b}\right)$ flow velocity along the boat track of the Kubu bifurcation on 27 March 2014 (b)

sured approaching discharge averaged over the tidal cycle is $3100 \mathrm{~m}^{3} / \mathrm{s}, 10 \%$ of the approaching discharge flows into the Kubu, while $90 \%$ of the discharge continues along the Kapuas Besar (Figure 6.10b). The half range of the tidal discharge is $2754 \mathrm{~m}^{3} / \mathrm{s}$. Although the tidal half range is less than the river discharge, the flow in the main channel to reverse shortly during flood tide due to the asymmetry of the tide (Figure 6.10). The strength of both the river and the tidal flow are lower in the side branch. The river flow is relatively stronger than the tide in comparison to the main channel so that the flow in the side branch does not reverse. This is expected from the higher damping of the tide in the longer and shallower side branch. The cross-sectionally averaged velocity of the downstream transect is not representative for the main branch, as the flow diversion is not complete at this point. The velocity in the main branch leads HW by 1:40 h, $24^{\circ}$ of the diurnal cycle. The side channel lags HW by $3: 40 \mathrm{~h} 49^{\circ}$. The lower velocity in the side branch, the relatively stronger river flow, and the large phase lag are expected since the Kubu channel is much shallower and longer than the main river channel.

\section{Flow}

Across the upstream transect of the approaching channel, the flow velocity gradually increases towards the left bank, from where the Kubu channel 

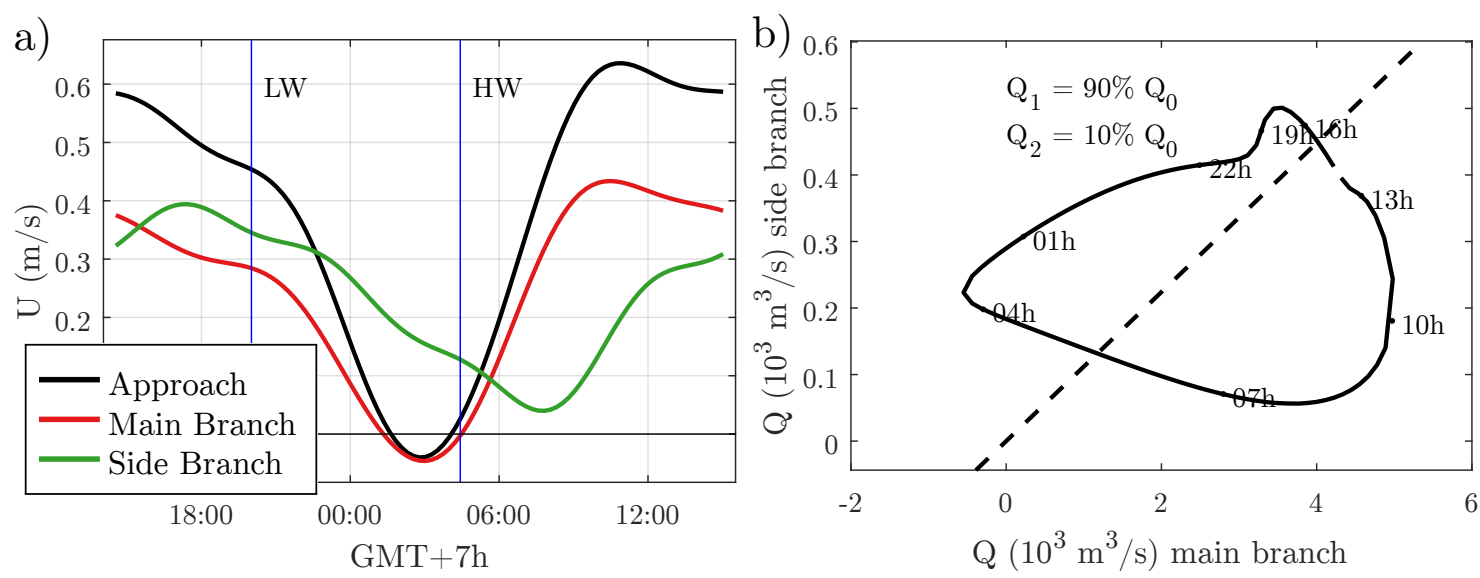

Figure 6.10: Cross-sectionally averaged flow velocity (a) and total discharge through the branches of the Kubu bifurcation (b) on $27^{\text {th }}$ March 2014, vertical line indicates the time of high water.

branches off (Figure 6.11). This is consistent with the increase of the depth towards the left bank. The velocity near the bed and near the surface increases similarly towards the left bank (Figure 6.12a). There is no apparent secondary circulation. Across the entire section, the transverse flow is directed towards the left bank and the vertical flow is downwards. The relative strength of the tidal flow with respect to the river flow increases from the left towards the right bank so that the flow only reverses in the right half of the cross-section during flood, but not in the left half (Figure 6.13). Across the downstream transect of the main branch, the velocity increases from right to left for $4 / 3^{\text {rds }}$ of the section, before it decreases over the submerged levee. Finally, near the left bank, the flow reverses into the Kubu channel (Figure 6.11).

The maximum velocity near the inlet is slightly lower than in the upstream transect, as a part of the water has already been diverted. The magnitude of transverse flow velocity is lower in the rightmost $3 / 4^{\mathrm{er}}$ of the transect than in the approaching channel. The near-bed flow is already directed to the right bank, anticipating the right turn of the main channel further downstream (Figure $6.12 \mathrm{~b}$ ). The vertical flow is upwards and weaker than in the transect of the approaching channel, where the flow near the bed is relatively stronger. The relative strength of the tidal flow with respect to the river flow increases towards the right bank, as in the transect of the approaching channel, but the tidal flow is overall stronger than in the approaching channel due to the influence of the Kubu branch (Figure 6.13b). Over the submerged levee, the magnitude of the velocity is low and the depth-averaged flow is slightly directed towards the side branch. The flow near the surface is aligned with the 

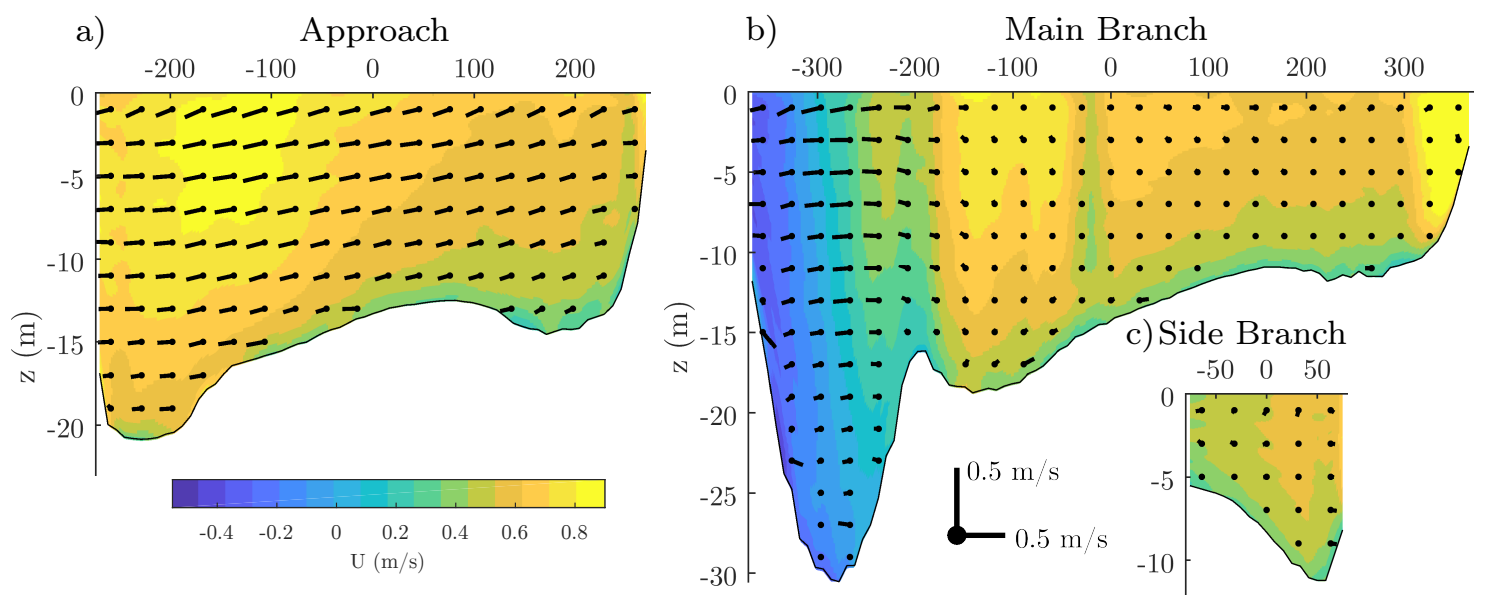

Figure 6.11: Flow velocity in the branches of the Kubu bifurcation during peak ebb tide on 27 Match 2014.
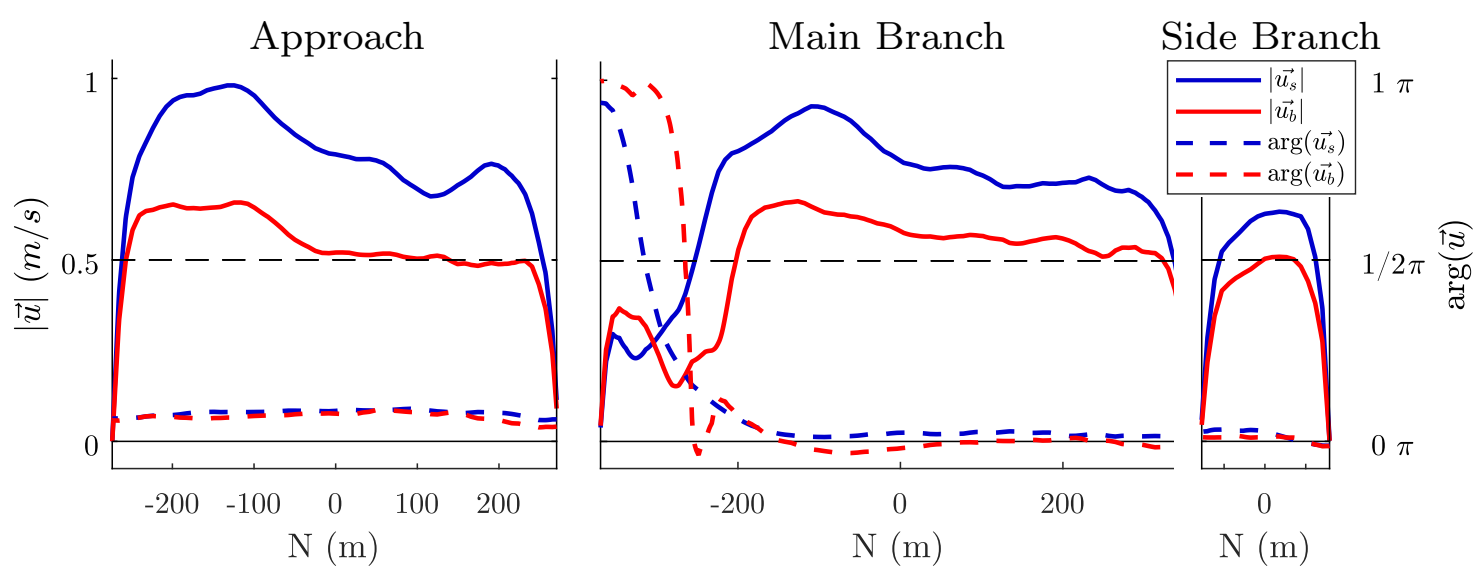

Figure 6.12: Flow velocity near the surface $\left(u_{s}\right)$ and near the bed $\left(u_{b}\right)$ across the branches of the Kubu bifurcation during peak ebb flow on 27 March 2014.

main branch, while the flow near the bed is directed towards the side branch. Near the left bank, the flow has already turned in direction of the Kubu channel. The velocity of the flow that has already turned is relatively strong near the bed so that vertical profile strongly deviates from a logarithmic profile (Figures $6.12 \mathrm{~b}$ and $6.9 \mathrm{~b}$ ).

There is upflow above the submerged levee and downflow near the left bank, which is consistent with the secondary circulation that is expected from flow curving into the side branch. In the downstream transect across the side branch, i.e. the Kubu channel, the flow velocity and shear stress are lower than in the Kapuas Besar but higher than directly at the diversion (Figures 6.4 and 6.5c). 


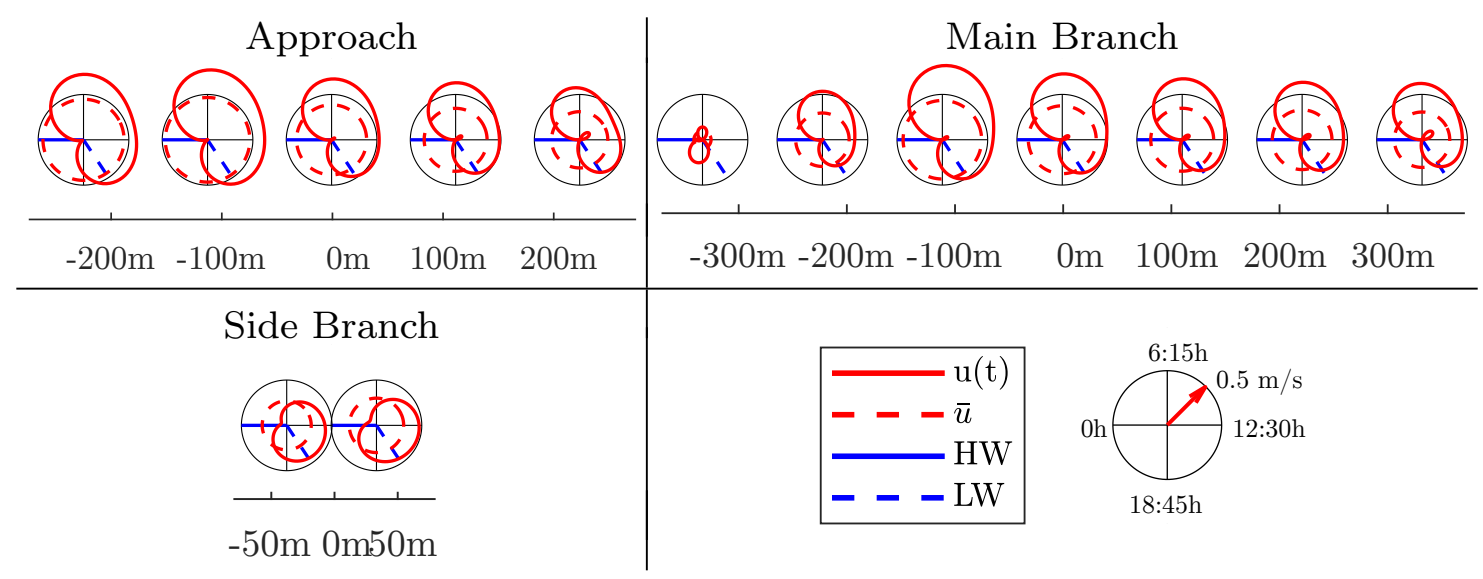

Figure 6.13: Flow velocity over the full tidal cycle of $25 \mathrm{~h}$ in intervals of $100 \mathrm{~m}$ for each crossing (bold red loops). Mean flow velocity is indicated by the dashed red circle. Time passes clockwise. Time of high water is indicated by the bold hand and time of low water by the dashed hand. Start time is shifted so that high water occurs at $0 \mathrm{~h}$.

\section{Pathway of suspended sand}

In the upstream transect, the concentration and depth-integrated transport of suspended sand increase from the left bank to the right over $2 / 3^{\text {rds }}$ of the cross-section, and then slightly decrease again towards the left bank (Figure 6.14a and Figure 6.15). The maximum of the sand concentration and transport is thus located closer to the channel centre than the maximum of the velocity, which is located more to the left. In the centre of the cross-section, the suspended sand reaches higher into the water column. There is a small local maximum of the concentration and transport in the smaller trench near the right bank. In the downstream transect of the main channel, the suspended sand distributed as in the transect of the approach (Figure 6.14b). The concentration increases from right to the left over $2 / 3^{\text {rds }}$ of the distance to the submerged levee and has a minor local maximum above the trench on the right bank. Left to the submerged levee, where the transect crosses the inlet of the side branch, the sediment concentration is higher in the upper part of the water column and decreases towards the bottom. The vertical distribution of the sediment concentration is very dissimilar to a Rouse profile. This is likely, because the suspended sediment enters the side branch over the submerged levee, where the upwelling convects it upwards. It is then convected farther across the side branch into the upper part of the water column by the transverse flow. Close to the bottom of the scoured inlet, suspended sand transport is weak. In the downstream transect of the side branch, the Kubu channel, 
the suspended sediment concentration is high (Figure 6.14c). In total $18 \%$ of the suspended sand is diverted to the side branch (Figure 6.15b). The division ratio for sediment is almost twice as large as the division ration of water. This is the opposite to the Kecil bifurcation, where proportionally less water than sediment enters the side branch.
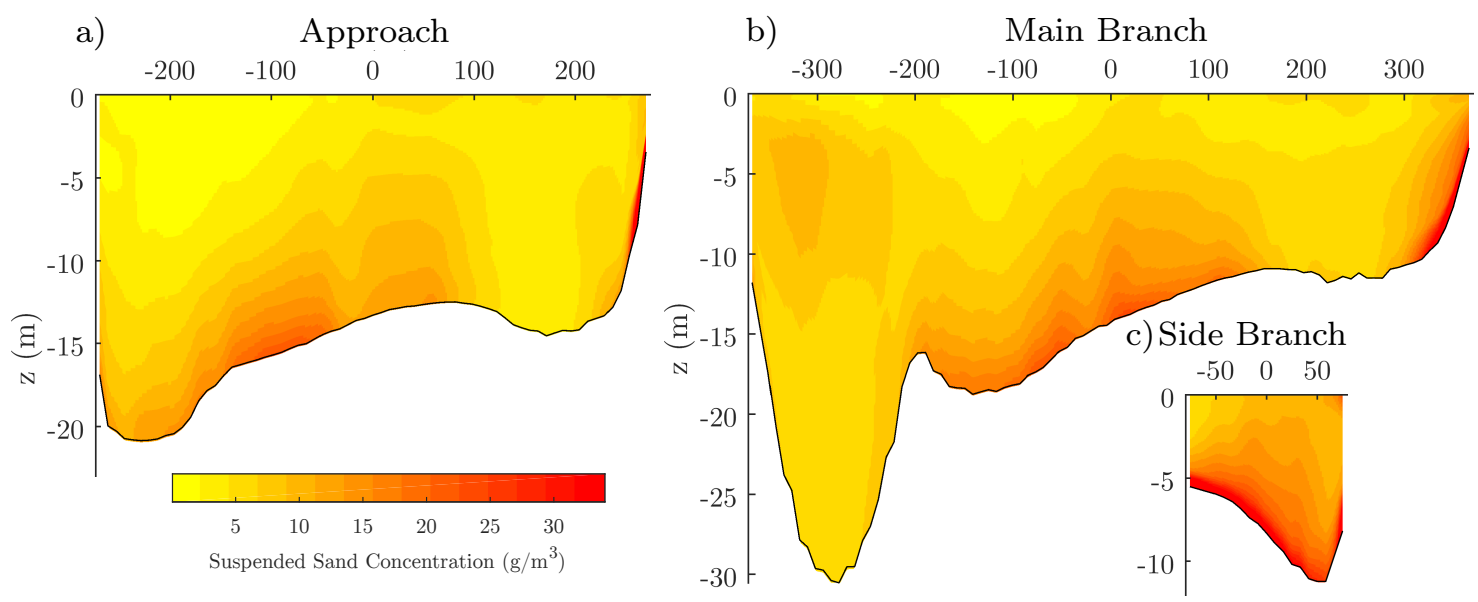

Figure 6.14: Suspended sand concentration in the branches of the Kubu bifurcation during peak ebb flow of 27 March 2014
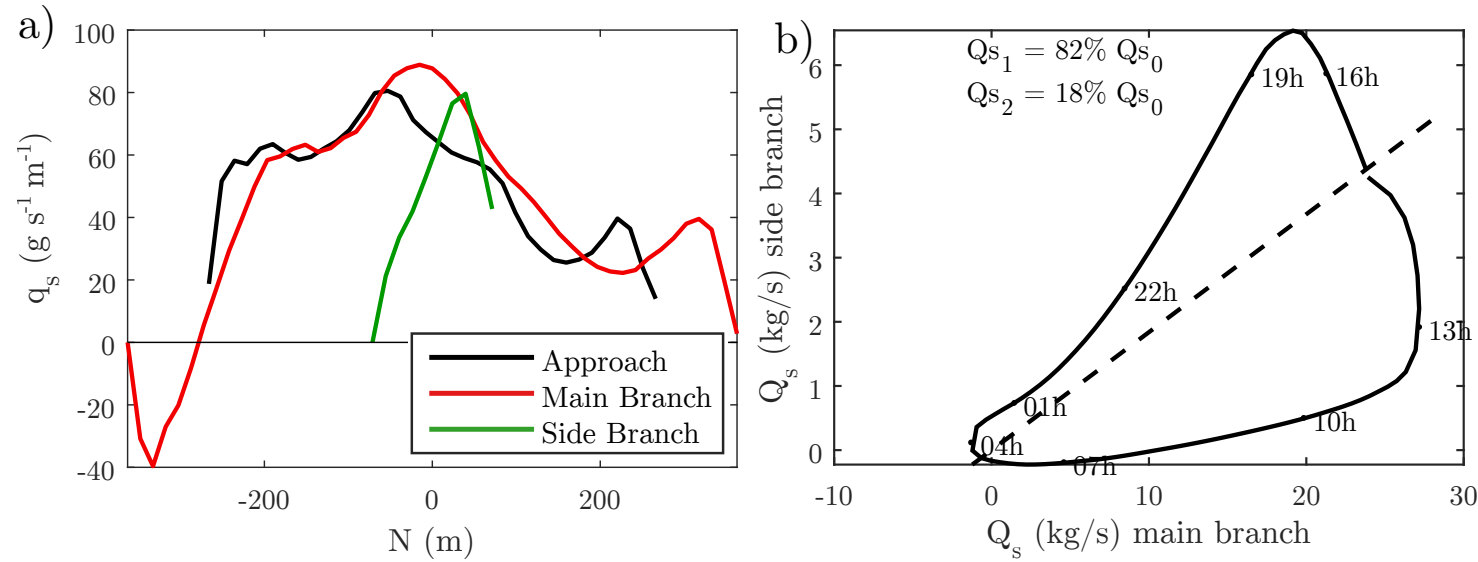

Figure 6.15: Depth-integrated suspended sand transport during peak ebb tide (a) as well as total suspended sand transport through the two branches over the tidal cycle (b) at the Kubu bifurcation on 27 ${ }^{\text {th }}$ March 2014 


\subsection{Discussion}

Laboratory and numerical experiments show that side branches receive a proportionally large fraction of bedload sediment (Bulle, 1926; Habermaas, 1935; Riad, 1961; Dutta et al., 2017). Often, side branches are therefore considered to be only ephemeral. The side branches of the two studied bifurcations of the Kapuas River did not diminish in size since the first hydrographic survey in 1882 and the Kapuas Kecil branch exists in its currents form very likely at least since the establishment of the city of Pontianak at its banks, 250 years ago. In this section, we discuss the factors that reduce the amount of diverted sediment.

\subsubsection{Bed morphology}

The diversion of flow strongly perturbs the bed geometry at the Kapuas bifurcations. The bed level rises at the bifurcations and drops at the downstream end into scour holes. Such scours are typical suspended load dominated rivers (Ikeda et al., 1987), and are often found at channel junctions (Ginsberg et al., 2009; Ferrarin et al., 2018). Scours are common in flow-diversions (Önen and Agaccioglu, 2013; Herrero et al., 2015) and chute cut-offs (Herbertson and Fares, 1991). The scour holes in the Kapuas extend into the side branches, similar to what has been observed in scale experiments (Herrero et al., 2015; Bulle, 1926; Riad, 1961). The examples from the Kapuas bifurcations show there is no inlet-step, which has been suggested as an important factor that can reduce sediment diversion into side channels (Slingerland and Smith, 1998). We think that inlet steps may be less relevant for avulsion dynamics than previously thought, as an initial step will rapidly erode due to the strong flow through the crevasse, and the small time scale of morphodynamic processes in the inlet region compared to those of the avulsion channel as a whole. Laboratory experiments have also shown that even at diversions with inlet steps, secondary currents can locally entrain the bedload material into suspension, and carry it into the side branch (Michelazzo et al., 2016).

At the Kapuas bifurcations, the bed also does not slope up across the approaching channel, towards the side branch. Such lateral bed slopes have been found to stabilize bifurcations in gravel bed rivers, and have been suggested to stabilize bifurcations in sand bed rivers as well (Bolla Pittaluga et al., 2015a). At an asymmetrical diversion, the approaching channel typically deepens on the side of the diversion channel, where the flow accelerates related to the longer path length to the side branch. On the opposite side of the di- 
version, the bed of the approaching channel rises, as the flow there has to decelerate because of continuity. This leads to the formation of shallow bars on the opposite bank of a chute cut-off (Zinger et al., 2013). The transverse slope model by Bolla Pittaluga et al. (2015a) is thus not applicable when one channel branches of to the side. At near-symmetrical river bifurcations, the downstream branches are typically shallower than the approaching channel (Kerssens and Van Urk, 1986), but this is not the case for the Kapuas. Rather, the bed level gradually decreases towards the sea (chapter 3). We attribute this to the tides. Upstream of the bifurcations, the tide is weaker than downstream, since part of the tidal energy is reflected back to the sea. Estimated by the method of (Lighthill, 2001), about 10\% tide is reflected at each of the bifurcations. The decrease in the river discharge at the bifurcations is thus compensated for by an increase of the tidal discharge.

\subsubsection{Flow}

At the Kapuas bifurcations, there are several locations where the vertical profile of the streamwise velocity strongly differs from the logarithmic shape that is expected from the law-of-the-wall. This is most pronounced in the immediate vicinity of the diversions, where the secondary flow is strong and where the water in the upper and lower parts of the water column flows into different directions. In the inner meander bend of the Kapuas Kecil bifurcation, the velocity is highest in the lower part of the water column. This is typical for sharp channel bends (Blanckaert and De Vriend, 2003), where the flow can separate from the outer bend (Leeder and Bridges, 1975; Ferguson et al., 2003). However, at the Kapuas Kecil bifurcation, the flow separates from the outer bend downstream of the diversion. Recirculation in outer bends with strong topography have also been observed in the Mahakam river (Vermeulen et al., 2015).

At the Kubu bifurcation, the vertical flow is in accordance with the theory of gradually varying flow (Song and Graf, 1994; Yang et al., 2006; CastroOrgaz and Hager, 2017), i.e. it is downwards in the approaching channel, where the bed level rises, and upwards in the downstream transect, where the bed level drops. There is no such pronounced vertical flow at Kapuas Kecil bifurcation, although the bed level does rise. Remarkable is also the absence of a clockwise secondary circulation in the approaching channel at the Kapuas Kecil bifurcation. This is likely caused by the diversion of the flow, which drives an anticlockwise circulation and thus counteracts the clockwise circulation of the meander bend. This reduces the strength of the secondary flow and rises 
the bed level at the bifurcation. The effective widening of the channel due to the bifurcation also contributes to the local rising of the bed level, which, to a smaller extent also occurs at the Kubu bifurcation. The diversion also influences the transverse velocity.

\subsubsection{Division of sediment}

At lateral diversions, a proportionally larger fraction of sediment than water is diverted to the side branch (Bulle, 1926; Dutta et al., 2017). This is indeed the case at the Kubu bifurcation, but not at the Kapuas Kecil bifurcation. The Kapuas Kecil receives a smaller fraction of sediment, which seemingly relates to the fact it branches off in an outer bend. Branches that are located in outer bends receive comparatively little sediment, primarily because of secondary currents (Raudkivi, 1993; Kleinhans et al., 2008). However, secondary circulation does not directly divert the sediment away from the Kapuas Kecil, as it is inhibited by the asymmetrical diversion geometry. Also, there is only a net transport of sediment towards the inner bend along the entry reach of a meander bend, where the path through the inner bend is shorter (Rozovskii, 1957; De Vriend, 1981; Kalkwijk and Booij, 1986). Once the transverse slope is fully established, which seems to be the case at the Kapaus Kacil bifurcation, there is no net transverse transport any more (Ikeda et al., 1987).

Suspended load dominated rivers can even carry the bulk of the sediment along the outer bend, when the bed material is uniform (Talmon, 1992), as the secondary flow advects momentum towards the outer bank (Ottevanger et al., 2012) so that the shear stress is highest in the outer bend (Fong et al., 2009). In contrast, the concentration of sediment in the outer bend at the Kapuas Kecil bifurcation is very low, although the near-bed velocity is of similar magnitude as in the channel centre, where the sediment concentration reaches its maximum. This can bne best explained by a coarsening of the bed material towards the outer bend. Local sorting and armouring thus most likely influence the sediment division at bifurcations, which has previously been established for the Rhine bifurcations (Sloff and Mosselman, 2012). The thalweg of the main channel at the Kapuas Kecil bifurcation is located about $1 / 5^{\text {th }}$ of the cross-section away from the outer bank. The bed has to be armoured over this stretch, as the bed slopes up over this stretch towards the outer bank.

Sorting of bed material also reduces the diversion of sediment into the Kubu branch, albeit to a slightly smaller extent. There, the sediment concentration decreases towards the left bank, from where the channel branches off, even though the region of high velocity nearly reaches the left bank. Indeed, the 
bed material of the Kapuas River varies strongly in space, and far outer bends typically consist of erosion resistant clay (chapter 3).

\subsubsection{Role of the inlet size}

A proportionally smaller fraction of sediment is diverted into a side branch when the local velocity of the diverted flow is low, as it controls the strength of the secondary flow. This is demonstrated by laboratory experiments where either the total discharge or the fraction of the diverted discharge is reduced (Herrero et al., 2015). The velocity can also be reduced by a widening of the inlet to the side branch at the location of the diversion. At artificial diversions, the amount of sediment diverted to the side can be controlled by the width of a weir that controls the flow into the side channel (Rosier et al., 2009). In natural bifurcations, the diversion can widen autogenously by the migration of banks. Previously, it has been reasoned that bank migration destabilizes the bifurcation, as the migration increases the angle at which the side channel branches off over time (De Heer and Mosselman, 2004). This is because the downstream bank of the inlet retreats and the upstream bank of the inlet advances. However, at the Kapuas bifurcations, the bank migration has not just resulted in a larger bifurcation angle, but also into a configuration in which the inlet section of the side channel is much larger than the cross-section further downstream (Figure 6.16). This is similar to levee breaches, which reach a size proportional to the discharge in the main channel (Michelazzo et al., 2018). The large inlet area reduces the strength of the diverted flow and therefore reduces the amount of diverted sediment. Sediment division ratios found at scale models with an idealized geometry and channels of constant width are thus do not necessarily transferable to natural bifurcations.

\subsection{Conclusion}

Measurements of flow and suspended sediment transport at two strongly asymmetrical bifurcations in a lowland river give new insight into the transport and division of sediment over distributaries:

The bathymetry of the bifurcations is very different from that of symmetric bifurcations and similar to that observed at scale models of lateral diversions. The thalweg of the main channel rises locally at the bifurcations and then plunges into a scour hole at the downstream end of the inlet to the side branch. The bed does not slope up across the approaching channel towards the side branch, but rather downward. Also, the bed does not feature a step at the 
a)

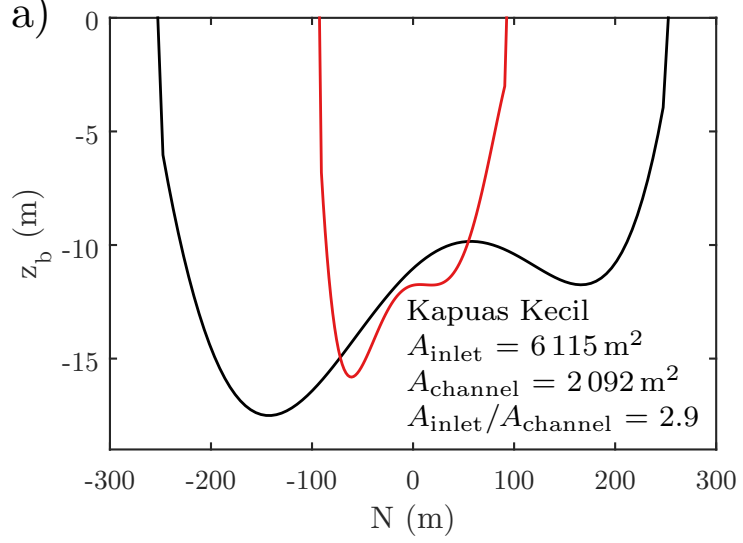

b)

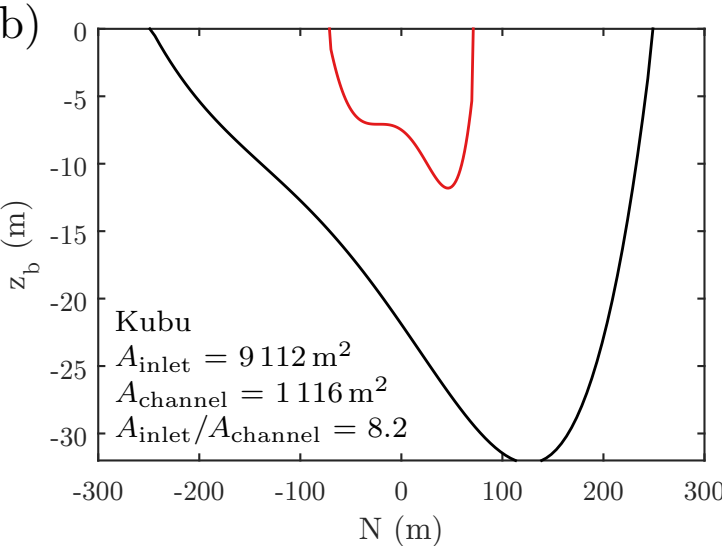

Figure 6.16: Cross-sections of the Kecil (a) and Kubu (b) side branches; the cross-section areas of the inlets (black) are much larger than that of the branches further downstream (red).

entrance of the side branch at the diversion. Common one-dimensional models that predict the sediment division based on the transverse slope or inlet steps are thus not applicable.

The bulk of the sediment bypasses the Kapuas Kecil, which branches off in an outer bend, whereas a larger fraction of sediment enters the Kubu channel, which branches of in a nearly straight reach. This confirms the influence of meandering of the stability of bifurcations. The observations at the Kapuas Kecil bifurcation show, that the amount of sediment diverted to the branch taking off from the outer bank is reduced primarily by coarsening of the bed material near the outer bank, rather than directly by the secondary flow. This is indicated by the low sediment concentration near the in the outer bend, despite the relatively high velocity near the bed, as well by the absence of a pronounced secondary circulation in the approaching channel.

The flow enters both side branches of the Kapuas River at low velocity, as the area of the inlet at the diversions is larger than that of the side branches further downstream. The large local width in the entrance region reduces the amount of the diverted sediment and therefore prolongs the lifespan of asymmetric side branches. 


\section{$7 \mid \begin{aligned} & \text { Role of the inlet width in the } \\ & \text { deflection of sediment to lateral } \\ & \text { branches of lowland rivers }\end{aligned}$}

The division of sediment at river bifurcations is crucial for the morpho1 dynamics of delta channel networks. Many natural bifurcations are strongly asymmetric so that a small channel branches off from the side of a large channel. The secondary currents at such an asymmetric bifurcation preferentially direct water that flows near the bottom towards the side branch. As the sediment concentration near the bottom is high, side branches receive a larger fraction of the approaching sediment load than of the water discharge. This causes side branches in scale experiments to fill in rapidly. However, there are many asymmetric bifurcations in river deltas that appear to be morphologically stable. This suggests that under certain conditions, less sediment is diverted than expected from small scale experiments. Recent surveys of bifurcations of the Kapuas River show that side branches can have entries that are much wider than their cross-sections further downstream. This may counteract sedimentation and increase their morphological stability. To test this hypothesis, we analyze the flow and sediment division at an idealized lateral diversion with a potential flow model. Our analysis confirms that a large inlet area moderates the fraction of the diverted sediment. We compare our findings to existing empirical relations derived from scale experiments and find that these do not necessarily scale up to the size of rivers.

This chapter is based on: Kästner, K., and A. J. F. Hoitink, The effect of the inlet width of lateral bifurcation branches on the division of sediment, Submitted to Journal of Fluid Mechanics, $2019 c$ 


\subsection{Introduction}

The division of sediment at river bifurcations determines the stability of delta channel networks. A branch that receives more sediment than what it discharges fills in, while a branch that receives less sediment scours and widens (Sloff et al., 2013). This can have dire consequences as channels can become defunct or infrastructure become undermined (Goodfriend and Stanley, 1999).

Many river bifurcations are strongly asymmetric so that a small channel branches off from the side of a large channel. This configuration is similar to that of man-made lateral diversions. Such diversions have historically been studied in the laboratory by Bulle (1926); Habermaas (1935); Riad (1961); Herrero et al. (2015), as well as recently with large scale computer simulations by Shettar and Murthy (1996); Neary et al. (1999); Gaweesh and Meselhe (2016) and Dutta et al. (2017). The experiments show that proportionally a larger fraction of the sediment load than that of the water discharge is diverted into side branches. This implies that side branches fill in rapidly over time, as the non-linearity of the sediment transport causes the transport capacity to decreases more rapidly than the size of a channel (Wang et al., 1995). However, there persist many asymmetric bifurcations in river deltas that have been stable over long periods of time. Results of scale experiments, both physical and numerical, therefore seem not to be directly transferable to river bifurcations.

The diversion of sediment in rivers is not necessarily similar to that in scale models. This is because some physical processes act differently on a larger scale, or because some processes play a role that are omitted in the model. One important process is the migration of river banks. Model channels have typically fixed banks and a regular geometry so that they intersect with sharp corners. This entails different flow and sediment transport patterns than in natural channels, where the transition between channels is smooth. A recent survey in the Kapuas River has furthermore shown that the entry section of a branch can have a much larger area than the cross-section of the branch has further downstream (chapter 6). The wide inlet could moderate the amount of the diverted sediment, and thereby promote the longevity of the side branches (chapter 3).

Several empirical relations for the division of sediment over bifurcation branches have been proposed. The are based on scale experiments (Raudkivi, 1993; Meijer and Ksiazek, 1994; van der Mark and Mosselman, 2013). When sediment is transported as bed load, then the division of sediment is related to the streamlines of the flow (Raudkivi, 1993). The dividing streamlines of both the depth-averaged and near-bed flow curve towards the diversion, but 
the secondary flow causes the arc near the bed to be larger. van der Mark and Mosselman (2013) considered this in their empirical model, but based their relation still on scale experiments. So far, no proper theoretical analysis of the dividing flow has been published. In this paper, we take the first step towards a better theoretic understanding of the flow and the consequential sediment partition at lateral diversions.

The flow at lateral diversions closely resembles potential flow (Hager, 1984). Potential flow has been applied to determine the velocity at lateral diversions by Modi et al. (1981); Sinha and Odgaard (1996); Kacimov (2000) as well as by Hassenpflug (1998). These studies focussed on the computation of the depth averaged flow, but neither explored the near bed flow nor its parameter space. Nevertheless, the near bed flow and the sediment division can be readily approximated from the depth-averaged flow (Raudkivi, 1993; Kawai et al., 1993). In this paper, we determine the division of sediment at a bifurcation with idealized geometry based on potential flow. This allows us to determine the parameters that are relevant for the division, and to compare the asymptotic behaviour with that of existing empirical relations. In particular, we investigate the role of the width of the entry reach of the lateral branch.

\subsection{Flow at lateral diversions}

We determine the flow at a bifurcation with highly idealized geometry, where the main channel has a constant width and a horizontal bottom. We represent the inlet to the side branch, the diversion, as a rectangular section with zero with the same depth as the main channel. We neglect the effect of friction and secondary flow on the momentum balance so that the depth-averaged flow is determined by continuity alone:

$$
\nabla \cdot(\mathrm{h} \overrightarrow{\mathrm{u}})=\frac{\partial \mathrm{hu}}{\partial \mathrm{x}}+\frac{\partial \mathrm{hv}}{\partial \mathrm{y}}=0
$$

where $\mathrm{v}$ is the velocity component pointing in the direction of the main channel, $y$ is the orthogonal velocity component pointing across the main branch towards the side branch. $\mathrm{x}$ and $\mathrm{y}$ are the corresponding coordinates, along and across the main branch respectively, and $h$ is the channel depth.

We consider flow with a small Froude number and hence neglect the perturbation of the surface elevation by the flow. The boundary conditions are 


$$
\begin{aligned}
\mathrm{u}(-L / 2, \mathrm{y}) & =\mathrm{u}_{0}+\frac{1}{2} \frac{\mathrm{w}_{s}}{\mathrm{w}_{0}} \frac{\mathrm{v}_{0}}{\mathrm{u}_{0}}, \\
\mathrm{u}(L / 2, \mathrm{y}) & =\mathrm{u}_{0}-\frac{1}{2} \frac{\mathrm{w}_{s}}{\mathrm{w}_{0}} \frac{\mathrm{v}_{0}}{\mathrm{u}_{0}}, \\
\mathrm{v}\left(\mathrm{x},-\mathrm{w}_{0}\right) & =0 \\
\mathrm{v}(\mathrm{x}, 0) & =\mathrm{v}_{0} f
\end{aligned}
$$

where $L$ is the length and $\mathrm{w}_{0}$ the width of the main channel. $\mathrm{w}_{s}$ is the width of the inlet to the side branch, $f$ is the velocity profile across inlet $\left(\int_{-\infty}^{\infty} f \mathrm{~d} x=\mathrm{w}_{s}\right)$ and $\mathrm{v}_{0}$ velocity averaged across the inlet. We only consider the limit case of a long channel $L \rightarrow \infty$ and centre the diversion at $x=0$.

The velocity is uniquely determined by the univariate velocity potential $\Phi$ with $\mathrm{u}=\frac{\partial \Phi}{\partial \mathrm{x}}, \mathrm{v}=\frac{\partial \Phi}{\partial \mathrm{y}}$ so that the continuity equation (7.1) simplifies to an equation in one variable:

$$
\nabla \mathrm{h} \cdot \nabla \Phi+\mathrm{h} \Delta \Phi=0 .
$$

For uniform depth, this simplified further to the Laplace equation $\Delta \Phi=0$. We reduce the number of parameters by normalizing distance by $\mathrm{w}_{s}$ and time by $\mathrm{w}_{s} / \mathrm{u}_{0}$ so that

$$
\begin{aligned}
\Phi & =\mathrm{u}_{0} \mathrm{w}_{s} \Phi, \\
\overrightarrow{\mathrm{u}} & =\mathrm{u}_{0} \vec{u}, \\
\overrightarrow{\mathrm{x}} & =\mathrm{w}_{s} \vec{x} .
\end{aligned}
$$

As potential flow is linear, it can be decomposed into the sum of an arbitrary combination of potential flows. This implies that the depth-averaged flow along the main channel does not influence the depth-averaged flow into the side branch and vice versa (figure 7.1). The potential flow can thus be determined by convolving the fundamental solution of the Laplacian with the velocity profile $f$. For the limit case of an infinitely wide main channel $\left(\mathrm{w}_{0} \rightarrow \infty\right)$ this is:

$$
\Phi=x-\frac{\alpha}{\pi} \int_{-1 / 2}^{1 / 2} f\left(x^{\prime}\right) \log \sqrt{\left(x-x^{\prime}\right)^{2}+y^{2}} \mathrm{~d} x^{\prime} .
$$

The parameter $\alpha=\frac{\mathrm{v}_{0}}{\mathrm{u}_{0}}$ is the relative strength of the flow into the side branch with respect to that along the main channel. This yields the first important result, namely that for a fixed velocity profile $f$, the depth-averaged flow is fully determined by a single parameter $\alpha$, irrespectively of the scale. 
There is an analytic solution when the velocity distribution $f$ is a piecewise continuous polynomial. In particular, for a constant profile $f=1$, the velocity is

$$
\begin{aligned}
& u=1-\frac{\alpha}{2 \pi} \ln \left(\frac{1+4 x^{2}+4 y^{2}+4 x}{1+4 x^{2}+4 y^{2}-4 x}\right), \\
& v=-\frac{\alpha}{\pi} \arctan 2\left(\frac{4 y}{4 x^{2}+4 y^{2}-1}\right) .
\end{aligned}
$$

Where $\arctan 2$ is the unambiguous two-argument arctangent with the range of the full circle of $2 \pi$. In the vicinity and far away from the bifurcation, the velocity asymptotically assumes simpler forms, which we consider hereafter.

a)

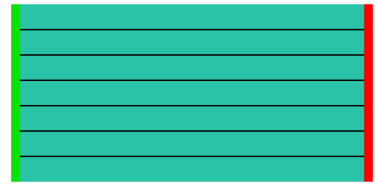

b)

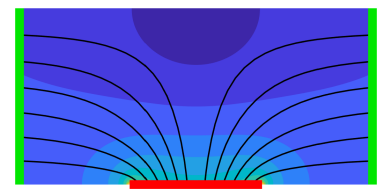

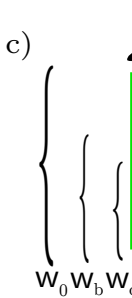

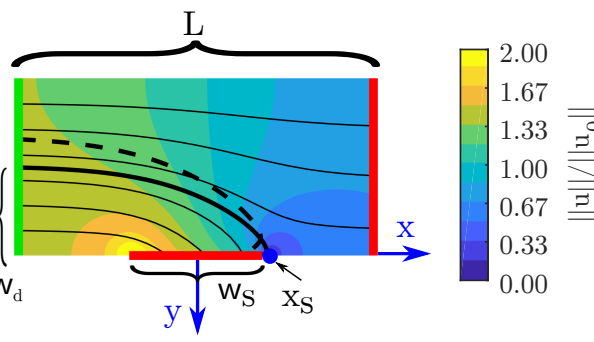

Figure 7.1: Potential flow at a lateral diversion c) as the superposition of the flow along the main branch a) and into the diversion b), dividing streamlines of the depth-averaged flow are bold, that of the near-bed flow dashed; red boundaries indicate outflow, green boundaries inflow

\section{Properties of the flow}

Linearized, the velocity near the centre of the diversion is

$$
\begin{aligned}
& u=1-\frac{4 \alpha x}{\pi}, \\
& v=\alpha\left(1+\frac{4 y}{\pi}\right) .
\end{aligned}
$$

The velocity component in the direction of the main channel thus decreases linearly along the diversion, and the transverse velocity directed towards the side branch increases linearly towards the diversion (figure $7.2 \mathrm{a}$ and $\mathrm{b}$ ). The velocity in the direction of the main channel $u$ does not change across the main channel at the centre of the diversion. 
Farther away from the diversion, the velocity approaches with increasing distance the hyperbolic asymptotes

$$
\begin{aligned}
& u=1-\frac{\alpha x}{\pi\left(x^{2}+y^{2}\right)}, \\
& v=\frac{\alpha y}{\pi\left(x^{2}+y^{2}\right)} .
\end{aligned}
$$

Far from the diversion, the velocity is thus identical to that caused by the outflow at a single point, and therefore neither depends on the width nor on the velocity profile along the diversion.

The flow along the main channel is symmetric, i.e. $u_{0}-u(x, y)=u(-x, y)-$ $u(0)$. The approaching flow gently accelerates towards the diversions (equation 7.8a), then rapidly decreases along the diversion (equation 7.7a), and gently recovers to the free stream velocity downstream of the diversion (equation 7.8a). The flow across the main channel is likewise symmetric $v(x, y)=$ $v(-x, y)$ (figure $7.2 \mathrm{~b})$. It decreases linearly across the main channel close to the diversion (equation 7.7b) and vanishes asymptotically (equation 7.8b). The velocity along the main channel has two poles, i.e. it spikes towards infinity at the downstream end and towards negative infinity at the upstream end of the diversion. There is thus a stagnation point at the bank downstream of the diversion, where the velocity is zero. The surface elevation decreases toward the diversion along and across the approaching channel, and then rises back along the diversion and reaches a maximum at the stagnation point, but it is not symmetric (figure $7.2 \mathrm{~b}$ and $\mathrm{c}$ ). This is typical for lateral diversions (Paris et al., 2012).
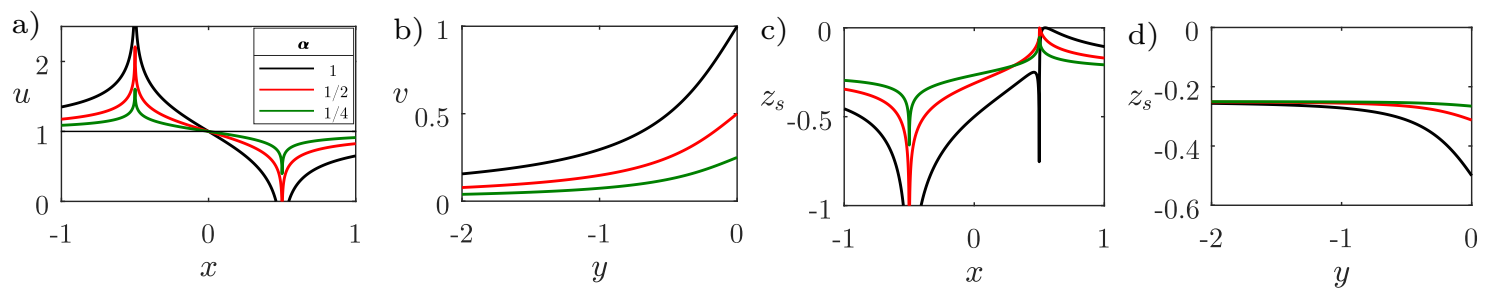

Figure 7.2: Flow velocity in the direction of the main channel along the diversion a), and in the direction of the side branch across the main channel b); water level along the diversion c) and across the main channel d), influence of secondary flow on the surface elevation $z_{s}$ is neglected 


\subsubsection{Dividing streamline of the depth-averaged flow}

The dividing streamline $\left(x_{s}, y_{s}\right)$ of the depth-averaged flow is given by the zeros of the equation

$$
\int_{0}^{y_{s}} u\left(x_{s}, y\right) \mathrm{d} y=\alpha \int_{-\infty}^{x_{s}} f(x) \mathrm{d} x .
$$

The integral can be solved analytically, but the zeros have to be determined numerically, as there is no closed form expression except for three special points. These are the origin of the streamline at the upstream end of the main channel $(-\infty, \alpha)$, the centre $\left(0, \frac{1}{2} \alpha\right)$, as well as the stagnation point downstream of the diversion $\left(\frac{1}{2} \operatorname{coth}\left(\frac{\pi}{2 \alpha}\right), 0\right)$. In general, the dividing streamline is located closer to the bank of the diversion, when the flow into the side branch is weaker, with respect to the approaching flow (figure 7.3a). There is always a stagnation point so that the flow reverses over a short distance downstream of the diversion. The stagnation point is located farther downstream of the diversion, when the flow into the diversion is stronger, with respect to the approaching flow (figure 7.3b). The flow reversal at the downstream corner of the diversion is due to warping of the streamlines of the inviscid potential flow, which is thus not a separation vortex.
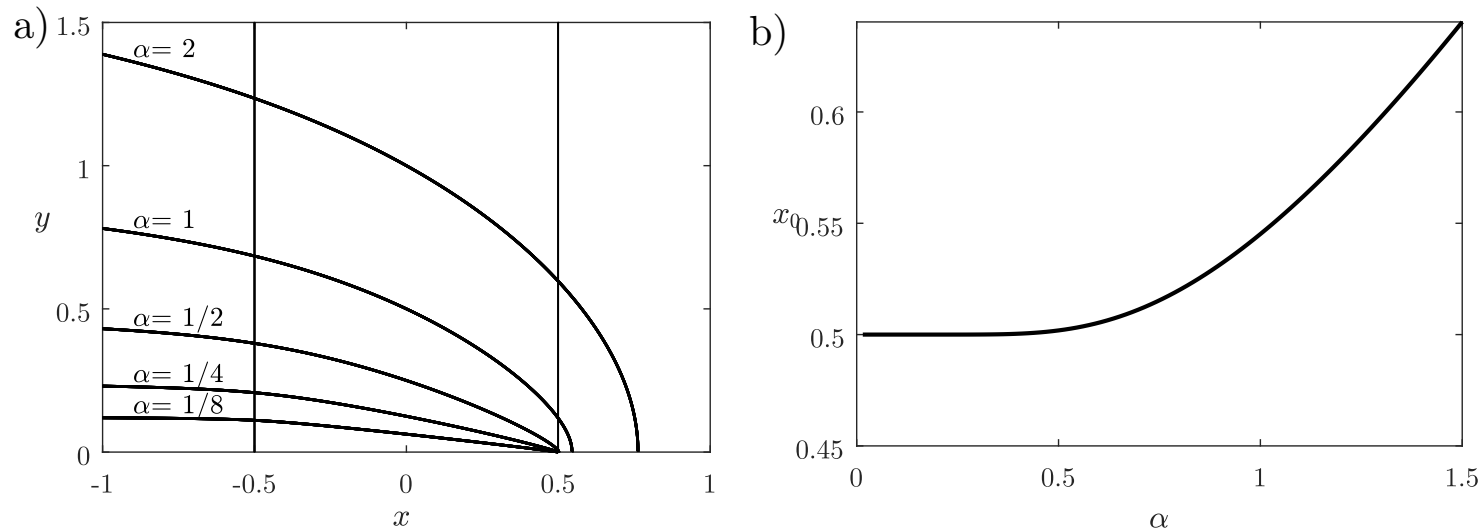

Figure 7.3: Dividing streamline of the depth-averaged flow (a) position of the stagnation point $\left(x_{s}, 0\right)(\mathrm{b})$ as a function of the relative strength of the diverted flow $\alpha=\mathrm{v}_{0} / \mathrm{u}_{0}$

\subsubsection{Velocity near the bed}

The velocity of the flow near the bed is (Rozovskii, 1957)

$$
\overrightarrow{\mathrm{u}} \mathrm{b}=\overrightarrow{\mathrm{u}}+f_{s} \frac{\mathrm{h}}{\mathrm{R}}\left(\begin{array}{c}
\mathrm{v} \\
-\mathrm{u}
\end{array}\right) \text {, }
$$


where $f_{s}$ is the secondary flow scale. Its value is 12 for sand bed rivers where the roughness is low (De Vriend, 1977). The relation does not account for the adaptation of the secondary flow to changes in curvature (Kalkwijk and Booij, 1986). We assume that the adaptation length is negligible for wide diversions with respect to the simplifications already made. Expressed in the non-dimensional quantities, the velocity near the bed is

$$
\vec{u}_{b}=\vec{u}+\beta \frac{1}{R}\left(\begin{array}{c}
v \\
-u
\end{array}\right)
$$

where $\beta=f_{s} \frac{\mathrm{h}}{\mathrm{w}_{s}}$. The normalized curvature $1 / R=\mathrm{w}_{s} / \mathrm{R}$ is (De Vriend, 1977)

$$
\frac{1}{R}=\frac{1}{|\vec{u}|^{3}}\left(\frac{\partial v}{\partial x} u^{2}+\left(\frac{\partial v}{\partial y}-\frac{\partial u}{\partial x}\right) u v-\frac{\partial u}{\partial y} v^{2}\right)
$$

Where $|\vec{u}|=\sqrt{u^{2}+v^{2}}$ is the velocity magnitude. The curvature can be analytically determined from the velocity, but its expression is lengthy. However, it only depends on the parameter $\alpha$. The velocity near the bed is thus uniquely determined by the relative strength of the diverted flow $\alpha$ and the secondary flow scale $\beta$.

The curvature of the dividing streamline increases towards the diversion, as the flow is initially parallel to the banks but directed towards the diversion at the bifurcation. For strong outflow, the dividing streamline resembles an arc along which the curvature continuously increases up to the stagnation point (figure 7.4). However, for weak outflow, the dividing streamline is nearly straight and only bends at two locations, once at the upstream end and once at the downstream end of the diversion. At the known point of the streamline where it intersects the centreline $(x=0)$, the curvature is

$$
\frac{1}{R_{0}}=\frac{8 \alpha^{2}\left(1-\frac{2}{\pi} \operatorname{atan}(\alpha)\right)}{\pi\left(1+\alpha^{2}\right)\left(1+\alpha^{2}\left(1-\frac{2}{\pi} \operatorname{atan}(\alpha)\right)^{2}\right)^{3 / 2}} .
$$

The following paragraph shows that the sediment is divided similarly to the relative strength of the secondary flow at the centreline.

\subsubsection{Division of sediment}

Sediment transported as bed load is divided similarly to the ratio of diverted flow near the bed and near the surface (Raudkivi, 1993). As the secondary flow strengthens the flow towards the diversion near the bed, more water is diverted from the lower part of the water column than from the upper part. 

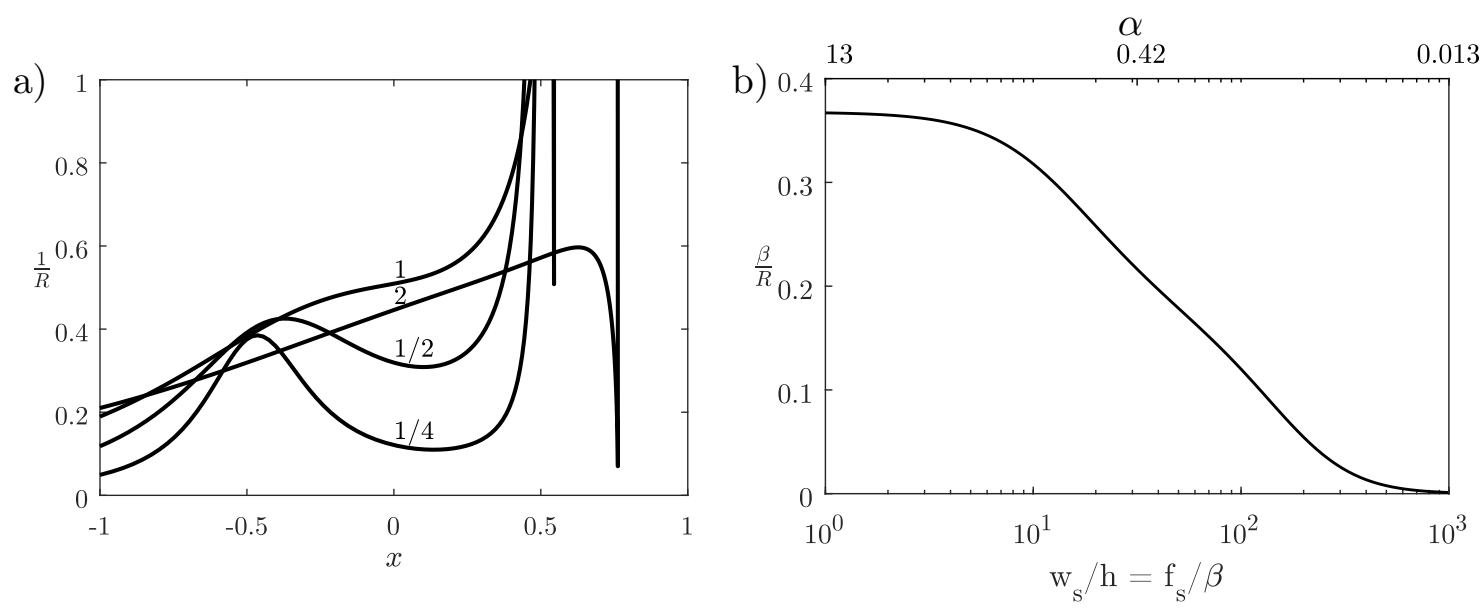

Figure 7.4: Curvature along the dividing streamline of the depth-averaged flow (a), and strength of secondary flow (b) at $x=0$, the intersection with the centreline, as a function of $\alpha$

The dividing streamline of the near-bed flow thus takes a wider turn than the depth-averaged flow (figure 7.5). The sediment-to-water division ratio is the ratio of the total amount of the diverted sediment per unit time $S_{s}$ to the total amount of the diverted discharge $Q_{s}$. This is essentially the ratio of the pseudo concentrations $c_{0}=S_{0} / Q_{0}$ in the approaching channel and $c_{s}=S_{s} / Q_{s}$ in the side branch. The water-to-sediment division ratio is equal to the distance of the dividing streamline of the near-bed $\left(y_{b s}\right)$ and the that of the depth-averaged flow $\left(y_{s}\right)$ at the upstream end of the main channel (figure 7.1):

$$
\frac{Q_{0}}{S_{0}} \frac{S_{s}}{Q_{s}}=\frac{c_{s}}{c_{0}}=\lim _{x \rightarrow-\infty} \frac{y_{b s}(x)}{y_{s}(x)}=\frac{\mathrm{w}_{b}}{\mathrm{w}_{d}} .
$$

For the depth-averaged flow, $\mathrm{w}_{d}=\alpha \mathrm{w}_{s}$. There is no simple analytic expression to determine the division line of the near-bed flow, and consequently neither for the sediment-to-water division ratio.

The dividing streamline of the near-bed flow $\left(x_{b}, y_{b}\right)$ can be determined by integrating it numerically backwards from the perturbed stagnation point:

$$
\begin{aligned}
x_{b} & =\int_{0}^{-\infty} u_{b} \mathrm{~d} t^{\prime}, \\
y_{b} & =\int_{0}^{-\infty} v_{b} \mathrm{~d} t^{\prime} .
\end{aligned}
$$

Alternatively, an initial point can be found by bisection.

Figure 7.5 shows the sediment-to-water division ratio as determined by the numerical integration of equation 7.15a. The sediment-to-water division ratio 

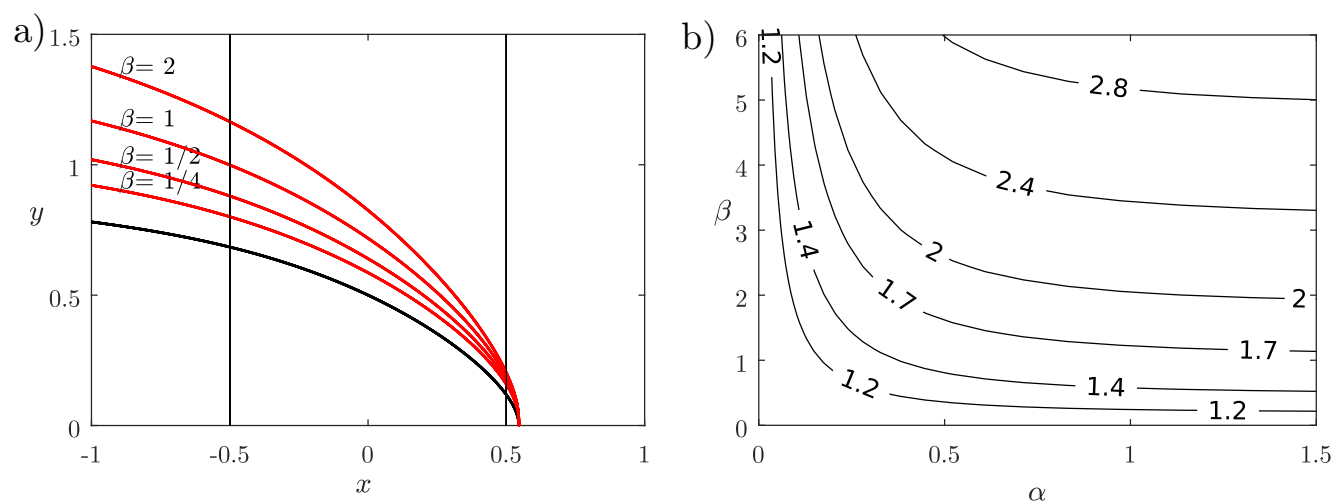

Figure 7.5: a) Dividing streamline of the near-bed flow for $\alpha=1 \mathrm{~b}$ ) Sediment to water division ratio $c_{s} / c_{0}$ at an idealized lateral diversion with an infinitely wide main channel

is always larger unity, i.e. proportionally always more sediment than water enters the side branch. The magnitude of the water-to-sediment division ratio is roughly proportional to the relative strength of the secondary flow

$$
\frac{S_{s} / S_{0}}{Q_{s} / Q_{0}}=\frac{c_{s}}{c_{0}} \approx 1+\beta \frac{1}{R_{0}} .
$$

The division of sediment is only a function $\alpha$ and $\beta$, as both parameters uniquely determine the near-bed flow. Although the division ratio just depends on two parameters, it is not a trivial function. It cannot be consistently approximated by a power law over the entire parameter range, as it depends on the radius of curvature $R$, which is a rational function (equation (7.13)).

\subsubsection{Effect of the width of the inlet to the side branch on the division of sediment}

The parameters $\alpha$ and $\beta$ depend on the size and the geometry of the inlet to the side branch. While $\alpha=\frac{Q_{s}}{h \mathrm{w}_{s}}$ is inverse proportional to its area, $\beta=f_{s} \frac{\mathrm{h}}{\mathrm{w}_{s}}$ is inverse proportional to its width-to-depth ratio. Both $\alpha$ and $\beta$ thus decrease with the width of the inlet to the side branch, as long as all other quantities remain equal.

As the strength of the secondary flow increases monotonously both with $\alpha$ and $\beta$, a wider inlet thus reduces the strength of the secondary flow, and thus the amount of the diverted sediment (figure 7.4b). For weak flow into the side branch $\left(\mathrm{w}_{s} \rightarrow \infty\right)$, the secondary flow strength approaches zero at the rate $\mathrm{w}_{s}^{-3}$ (figure $7.4 \mathrm{~b}$ ). For a very wide inlet to the side branch, the sediment-to-water division ratio thus approaches unity, i.e. the sediment is diverted in the same 
proportion as the water. For a narrow inlet to the side branch $\left(\mathrm{w}_{s} \rightarrow 0\right)$, the secondary flow strength reaches a finite limit (figure 7.4b). Thus, regardless of the inlet geometry, the amount of the diverted sediment remains finite. This is because for strong outflow at narrow diversions, the dividing streamline of the near-bed flow turns at a larger distance from the diversion, where the curvature of the flow is lower (figure 7.5a). An important consequence of the relaxation for strong outflow is, that a single power law cannot consistently predict the sediment-to-water division ratio both for strong and weak outflow. This is also apparent from the streamline curvature, as it is a rational function of the flow strength (equation 7.13).

\subsection{Discussion}

The potential flow model confirms that for the same discharge division, less sediment is diverted to the side branch, when the inlet is wider. This is in agreement with laboratory experiments. Sediment transport over side weirs can be entirely suppressed, when the weir is constructed wide enough (Rosier, 2007). Bulle (1926) already noticed, that smoothing of the diversion corners and the coincidental increase of the inlet area reduces the amount of the diverted sediment in scale experiments. Our model strongly simplifies the geometry of the bifurcation and determines the flow into the side branch by a predefined velocity profile, without explicitly including the side branch into the computational domain. This neglects the feedback of the flow within the side branch on the division. The feedback can be important when the flow in the side branch separates (Ramamurthy et al., 2007; Constantine et al., 2010). The separation bubble effectively reduces the inlet width and thus the amount of the diverted discharge and sediment. The contraction coefficient has to be considered for the design of artificial diversions. At alluvial bifurcations, flow separation can occur for a short period after a bifurcation is formed, i.e. by a chute-cutoff or an avulsion event. However, the banks align over time with the streamlines, so that the flow does not separate any more. Indeed, the flow does not separate within the side branches of the Kapuas, despite their large angles, at which they branch off (Kästner and Hoitink, 2019a). The representation of the flow by a velocity profile is therefore reasonable.

Although our model supports the hypothesis that a wide inlet to a side branch moderates the amount of the diverted sediment, it does not explain why and under which conditions side branches maintain a larger cross-sectional area at the diversion than further downstream. This requires a morphological model that incorporates bank migration. 


\subsubsection{Empirical sediment to discharge division ratios}

In this section, we put empirical relations for the division of sediment in perspective to that determined with the potential flow model. These relations typically predict the ratio of the diverted and the approaching flux of sediment $S_{s} / S_{0}$ depending on the ratio of the diverted to the approaching discharge $Q_{s} / Q_{0}$. These quantities lose their meaning in the limit case of a very wide main channel. Therefore, we recast the relations to predict the waterto-sediment division ratio, i.e. the ratio of the sediment concentration of the diverted flow to that of the approaching flow $c_{s} / c_{0}$ depending on the ratio of the velocity of the diverted flow with that of the approaching flow $\alpha=v_{0} / u_{0}$.

According to the potential flow model, the water-to-sediment division ratio 1) increases with the strength of the flow into the side branch, $\alpha$, which is inverse proportional to the inlet width, $\mathrm{w}_{s}$, and 2) increases with the secondary flow scale, $\beta$, which is proportional to the channel depth, $\mathrm{h}$, and inverse proportional to its width, $\mathrm{w}_{s}$. For practical relevance, empirical relations have to reasonably scale to the dimension of real rivers. Empirical relations are typically fit to scale experiments, but their consistency at the river scale is rarely validated. Regarding our findings, the water-to-sediment-division-ratio has to: 3) approach unity for weak flow into the side branch $\left.\left(\lim _{\mathrm{w}_{s} \rightarrow 0} c_{s} / c_{0}=1\right), 4\right)$ remain finite for strong flow into the side branch $\left(\lim _{\mathrm{w}_{s} \rightarrow \infty} c_{s} / c_{0}<\infty\right)$, and 5) be asymptotically independent of the width of the main channel $\left(\mathrm{w}_{0} \rightarrow \infty\right)$.

In his textbook on the diversion of sediment, Raudkivi (1993) gives the relation

$$
\frac{\mathrm{w}_{b}}{\mathrm{w}_{s}}=a_{b}+b_{b} \alpha,
$$

where $\mathrm{w}_{b}$ is the width of the diverted flow near the bed in the upstream channel, $a_{b} \approx 0.4$ and $b_{b} \approx 1.15$ are empirical parameters. This is equivalent to a sediment-to-water division ratio of

$$
\frac{c_{s}}{c_{0}}=a_{b} \frac{1}{\alpha}+b_{b} .
$$

Meijer and Ksiazek (1994) conducted numerical scale experiments for a diversion with idealized geometry. They suggest two relations. The first one is

$$
\frac{S_{s}}{S_{0}}=k_{0}+k_{1} \frac{Q_{s}}{Q_{0}},
$$

with the empirical values $k_{1}=2.63$, and $k_{0}=-0.1$ for the deflection of bed load at a $90^{\circ}$ diversion. The relation is asymptotically only asymptotically consistent 
when $k_{0}=0$ (Wang et al., 1995). In this case, the relation simplifies to

$$
\frac{c_{s}}{c_{0}}=k_{1}
$$

The second relation proposed by Meijer and Ksiazek (1994) is the power law,

$$
\frac{S_{s}}{S_{1}}=\left(\frac{Q_{s}}{Q_{1}}\right)^{m}\left(\frac{\mathrm{w}_{1}}{\mathrm{w}_{\mathrm{s}}}\right)^{n}
$$

with the empirical values $m=1.15$ and $n=-0.71$. The relation is asymptotically only consistent for $m+n=1$ (Wang et al., 1995). For a bifurcation with uniform bed level and constant width of the main channel, the relation is identical to a sediment-to-water division ratio of:

$$
\begin{aligned}
\frac{c_{s}}{c_{0}} & =\frac{1}{a}\left(\frac{\mathrm{w}_{1}}{\mathrm{w}_{0}}+\left(\frac{1}{a}-\frac{\mathrm{w}_{1}}{\mathrm{w}_{0}}\right)^{k}\right)^{-1} \\
& =c_{0} \frac{1}{a}\left(\frac{1}{a} \frac{Q_{1}}{Q_{0}}+\left(\frac{1}{a}\left(1-\frac{Q_{1}}{Q_{0}}\right)^{k}\right)^{-1}\right) .
\end{aligned}
$$

For a wide main channel, this approaches asymptotically

$$
\frac{c_{s}}{c_{0}}=\alpha^{m-1} .
$$

The potential flow model suggests $m>1$, which was also fit by Meijer and Ksiazek (1994) to their experimental data. Wang et al. (1995) derived stability criteria for symmetric bifurcations depending on the parameter $m$ and the degree of nonlinearity of the sediment transport formula.

van der Mark and Mosselman (2013) determine the sediment division by a sophisticated relation

$$
\frac{S_{s}}{S_{0}}=\frac{Q_{s}}{Q_{0}}+\left(c \frac{Q_{s}}{Q_{0}} \frac{\mathrm{w}_{0}}{\mathrm{w}_{s}}\right)^{b / 3} \frac{\mathrm{R}}{\mathrm{w}_{0}} \theta\left(\frac{1}{\cos \delta}-1\right),
$$

where $c$ is a constant, $b$ the degree of nonlinearity of the sediment transport, and $\theta, R$, and $\delta$ depend on the channel geometry and flow conditions as specified by van der Mark and Mosselman (2013). The corresponding sediment-towater division ratio is, with $\theta, R$, and $\delta$ being functions of $\alpha$ and $\beta$ :

$$
\frac{c_{s}}{c_{0}}=1+c^{b / 3} \alpha^{(b / 3-1)} \frac{R}{\mathrm{w}_{s}} \theta\left(\frac{1}{\cos \delta}-1\right) .
$$


We compare the performance of the relations qualitatively, i.e. only verify that their limit behaviour is consistent. Only the relations by Meijer and Ksiazek (1994)ii and van der Mark and Mosselman (2013) correctly predict the sediment to water division ratio to increase with the strength of the flow into the side branch $\alpha$ (figure 7.6). In contrast, the relation by Raudkivi (1993) predicts the ratio to decrease with the flow strength and seems thus not applicable to wide channels. The relation by van der Mark and Mosselman (2013) correctly predicts the sediment-to-water division ratio to go to unity for weak flow into the side branch, but the relation Meijer and Ksiazek (1994)ii predicts it to go to zero. For very weak outflow, the sediment division is limited by critical shear stresses, which is not included in the potential flow model, so that the sediment to water division ratio indeed could drop to below unity. Only the relation by van der Mark and Mosselman (2013) predicts that the sediment-to-water division ratio increases with the channel depth. However, it does not limit the amount of the diverted sediment when the diverted flow is strong so that in extreme cases more sediment is predicted to enter the side branch than actually approaches the diversion. This is not a severe limitation since the relations are only designed for subcritical flow. The models by Meijer and Ksiazek (1994)ii and van der Mark and Mosselman (2013) very differently predict the fraction of the diverted sediment with respect to the fraction of the diverted discharge. While the model by Meijer and Ksiazek (1994)ii predicts the sediment-to-water division ratio to be lower when more discharge is diverted, the model by van der Mark and Mosselman (2013) predicts the ratio to be higher when more discharge is diverted. The sediment-to-water division ratio is most likely a non-monotoneous function of the discharge division, as its value has to approach unity when either a very small fraction or almost all of the discharge is diverted so that neither model seems right. More insight can be gained through potential flow calculations with finitely wide channels.

The empirical relations either miss the dependence on the depth or are not consistent in the limit cases. Therefore, they most likely do not reliably predict the division of sediment in rivers. This probably originates in the low variability of the experimental data they are based on. The width of scale models is typically fixed, and the width-to-depth ratio is much lower than that of rivers. This gap could be addressed by numerical experiments, where the geometry is not scaled down. The empirical relation by van der Mark and Mosselman (2013) seems most promising, but it predicts that the streamline radius of curvature increases with the main channel width, which is only true as long as the main channel is narrow. The prediction of the sediment division can probably be improved, when this is addressed. 

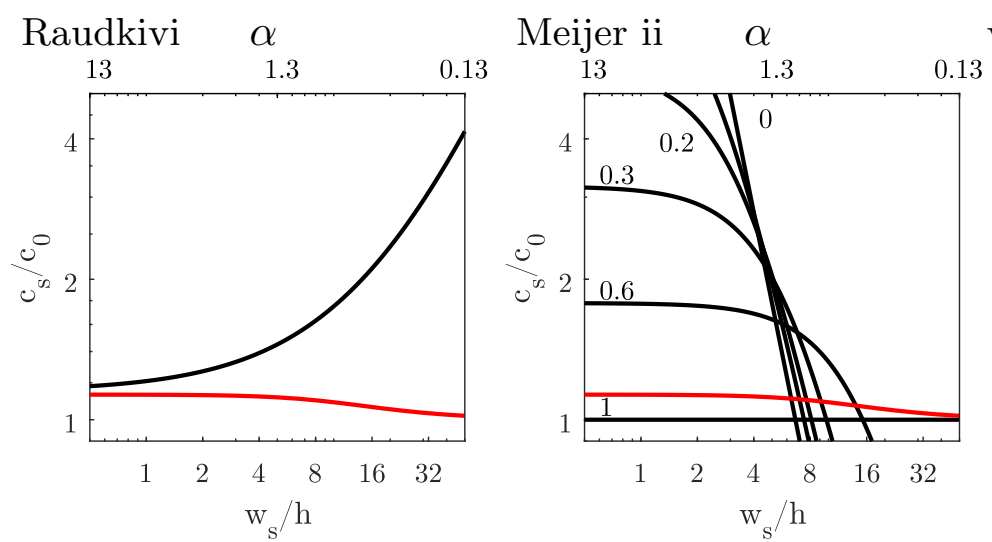

van der Mark $\alpha$

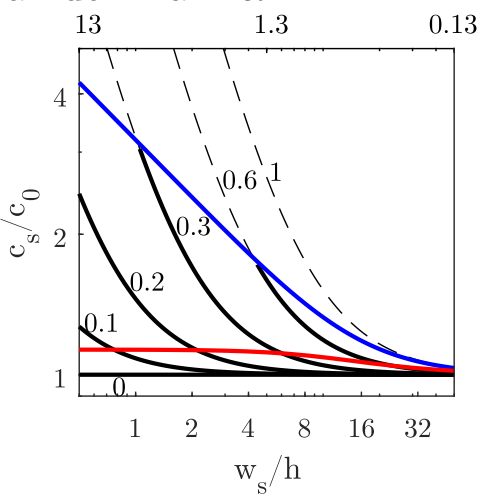

Figure 7.6: Sediment-to-water division ratio predicted by empirical relations (black) and by the potential flow model (red). Curves are numbered by the discharge division ratio $Q_{s} / Q_{0}$. The potential flow solution is only shown for an infinitely wide main channel $\left(Q_{s} / Q_{0}=0\right)$. Infeasible predictions $\left(S_{s}>S_{0}\right)$ are dashed, the blue line indicates the limit of feasibility $\left(S_{s}=S_{0}\right)$.

\subsection{Conclusion}

We present a mathematical analysis of the division of sediment at open channel bifurcations where one channel branches off to the side. In particular, we consider the idealized case of a wide main channel and a flat channel bottom. In this case, the depth-averaged flow at the diversion is determined by a single parameter. This parameter is the relative strength of the approaching flow and the diverted flow, and thus inverse proportional to the cross-section area of the side branch inlet. The division of sediment transported near the bed is uniquely determined in combination with a second parameter that is inverse proportional to the width-to-depth ratio of the side branch inlet. The potential flow model shows that for a given division of the water discharge, less sediment is diverted when the inlet to the side branch is wider. This agrees with recent observations in the Kapuas River, where wide inlets to side branches moderate the amount of the diverted sediment. Our analysis further reveals that existing empirical relations based on laboratory experiments do not consistently scale up to larger dimensions, and therefore cannot reliably predict the division of sediment in rivers. 


\section{$\mathbf{8}$ | Synthesis}

This thesis contributes to our knowledge of hydrodynamics and morphology of the reaches along the fluvial-tidal transition of large sand-bed rivers. It advances methods for monitoring as well as idealized modelling. It also gives insight into the discharge and sediment transport regime of the Kapuas River. This chapter highlights important results of this thesis and puts them into a wider context, relates them to recent developments in the field, and looks at future challenges.

\subsection{Morphology of the fluvial-tidal transition}

Chapter 3 shows that along-channel trends of the hydraulic geometry break between the fluvially and tidally dominated reaches of the Kapuas River. The Kapuas river does not gradually widen towards the sea, but rather narrows and deepens along the alluvial plain, only to widen up along the very last kilometres. Breaks of hydraulic geometry at the fluvial-tidal transition have also been found elsewhere, for example in the Mahakam (Sassi et al., 2012) and the Santee River, U.S.A. (Torres, 2017). However, even without the influence of tides, the river geometry can change towards the sea, due to backwater effects (Fernandes et al., 2016; Chatanantavet et al., 2012). For instance, a downstream narrowing has also been described for the Trinity River, Texas, U.S.A., which is not tidally influenced (Mason and Mohrig, 2018). The Trinity River also becomes morphologically less active towards the sea, which agrees with observations in the Kapuas (Vermeulen et al., 2016).

Further empirical evidence is required on how the fluvial-tidal transition and backwater affect the hydraulic geometry. This has to go hand in hand with the development of conceptual and idealized models. A major challenge in morphological modelling is to close the set of equations which determine the hydraulic geometry. There are at a first glance only two obvious equations, the continuity of discharge and sediment transport, but there are at least three unknowns: width, depth and slope. Often the closure is altogether circumvented 
by fixing one of the unknowns. Some models fix the width, as the migration of banks is inhibited in managed rivers (Bolla Pittaluga et al., 2015b). Other models fix the slope, as it changes at a much slower rate than width and depth (Blom et al., 2017). However, this approach merely determines the remaining quantities from the one that is specified a priori. An appropriate closure is thus required for the fluvial-tidal transition. One such closure is the assumption of a constant bankfull bed shear stress (Parker, 1978). However, recently Li et al. (2015) have shown that the bankfull bed shear stress is not constant. The conundrum might be resolved by properly modelling the bank migration. Bank migration models have made considerable progress recently (Eke et al., 2014; Stecca et al., 2017). The Kapuas River with varying migration rates along different reaches can serve as a realistic test case.

Idealized models predict the graded equilibrium of rivers (Zhou et al., 2017), where the slope and bed material grain size decreases gradually towards the sea. However, tectonic activity can disturb the graded profile and even rearrange the drainage network. The time between perturbations is not necessarily long enough for a river to reach a morphological equilibrium. The Kapuas River, for example, has not yet reached a graded profile (figure 1.6) since it broke through the Semitau range into the Melawi basin (Wing Easton, 1899; Ter Bruggen, 1935; Sibinga, 1953). The forming of a graded profile is impeded furthermore by heterogeneity of the catchment geology. Even for rivers where the drainage network has remained stable for long periods, such as the Rhine (Böhme et al., 2012), morphological features like the gravel sand transition are attributed to tectonic forcing (Frings, 2011). More importantly, many features of rivers are transient, i.e. they can only be observed while the river is not yet in an equilibrium and evolves over time. These include downstream fining, the gravel sand transition and delta-lobe switching, for example.

Observations of the Kapuas highlight yet another stark contrast between idealized models and reality: the channel geometry and bed material vary strongly over short distances. Trends of hydraulic geometry and in particular of grain size become only apparent after averaging over long distances. This is not unique to the Kapuas. Spatial sorting of bed material into patches along meander sequences has also been observed in the Rhine (Frings, 2011). Local variation of the bed material, in particular armouring in outer bends, can be important for the morphodynamics of the river as a whole (Sloff, 2010). 


\subsection{Suitability of HADCPs for discharge monitoring}

Chapter 2 shows that it depends on the field site whether the deployment of an HADCP for discharge monitoring is meaningful or not. Rasau, close to the sea, favours the deployment of HADCPs, as the discharge cannot be determined reliably from the stage alone. At Sanggau, the stage-discharge relation is reliable, and the index-velocity hardly improves the rating. This is because the HADCP measures the velocity only in a small part of the cross-section near the bank. The relationship between the measured and cross-section averaged velocity is complex and sensitive to perturbations. Sensitivity of HADCP measurements to local bathymetric features has recently also been shown elsewhere (Phan et al., 2014). Comparable performance of index-velocity and stage discharge rating has been shown as well (Cheng et al., 2018). The preference for the deployment of an HADCP at Rasau is not due to the perfection of the velocity measurement either, but rather due to the unreliability of the rating-curve. Uncertainty of HADCP measurements with respect to the tidal phase during flow reversal has recently also been observed (Kimura et al., 2011; Phan et al., 2015). Practical aspects further limit the usability of HADCPs: the instrument deployed at Sanggau was frequently obstructed by driftwood, became misaligned due to mooring boats and fell dry during low flow. The deployment is strenuous, and the instrument requires regular service.

However, even a small improvement of the monitored discharge may be worth the deployment of an HADCP at stations where water is valuable (McMillan et al., 2017). When an HADCP is deployed, the instrument should be deployed with care: rather in between bends than in a meander bend, out of the driftwood convergence zone, and sufficiently deep to measure during low flow. It should have a long enough range to span the entire cross-section, and if possible, should rather be deployed at a bridge pier near the channel centre than at a jetty near the bank. The accuracy can be increased by predicting the discharge in combination with a numerical model (Nihei and Kimizu, 2008), but even better is to turn to technologies that can cover the entire cross-section, such as modern travel time systems (Kawanisi et al., 2010) or radar (Cheng et al., 2015).

\subsubsection{Runoff and discharge of the Kapuas}

Between 2013 and 2015 discharge ranged between $10^{3}$ and $10^{4} \mathrm{~m}^{3} / \mathrm{s}$ at Sanggau, $285 \mathrm{~km}$ upstream from the sea (chapter 2). Rainfall-runoff derived from 
TRMM and GPM satellite data were calibrated with these measurements to obtain insight into the climatological mean (Bol, 2015; Hidayat et al., 2017). The survey period turned out to capture both high and low flow extrema. December 2013 was the wettest month between 1998 and 2015, while February 2014 was the driest month observed in Singapore since commencement of records in 1869 (Meteorological Service Singapore, 2014). The Kapuas did not flow over the banks downstream of Sanggau during high flow in 2014. Overbank flow is thus a rare event, but occurs infrequently, since at one place houses that had been erected after the transmigration in the 1970s with the floor on ground level had been abandoned to move to houses with an elevated floor.

With the calibrated rainfall-runoff model, global databases and models can be benchmarked. The annual average of the discharge of the Kapuas at Sanggau between 2000 and 2015 was $5900 \mathrm{~m}^{3}$ /s (Hidayat et al., 2017). The value of $6500 \mathrm{~m}^{3} / \mathrm{s}$ given by van Bemmelen (1949) is slightly higher, which can be explained by runoff downstream of Sanggau. The discharge predicted by the WBMsed model is by $900 \mathrm{~m}^{3} / \mathrm{s}$ lower than the predictions of the rainfall-runoff model for the period of 2000 to 2010, where the time series overlap (Cohen et al., 2013). The discharge of $3200 \mathrm{~m}^{3} / \mathrm{s}$ given by Milliman and Farnsworth (2013) and Meybeck and Ragu (1995) seems far too low compared to our measurements and predictions by runoff models. Trends due to climate and land use change can be determined by comparing the rainfall with historic measurements. Precipitation derived from satellites for the entire catchment $\mathrm{Hi}$ dayat et al., 2017) do neither reveal a change of the annual precipitation nor a change of the seasonal pattern since the end of the $19^{\text {th }}$ century (Enthoven, 1903). Between 1998 and 2015, the yearly precipitation averaged over the catchment was $3646 \mathrm{~mm}$, whereas between 1879 and 1899 the precipitation in Sintang was $3734 \mathrm{~mm}$ per year (figure 8.1). The interannual standard deviation of $453 \mathrm{~mm}$ pooled from the 39 years of measurement. The average difference of $88 \mathrm{~mm}$ per year is small compared to the standard error of $145 \mathrm{~mm}$ and thus not significant. The shorter discharge time series predicted by the rainfall-runoff models and the WBMsed model do neither show significant trends. The historic measurements hence do not support a decrease of precipitation, as claimed by Kumagai et al. (2013a) and Herawati et al. (2017). However, changes in precipitation may simply not be extractable from the limit data, as the historic measurements were only taken at one location and no measurements are available for the largest part of the $20^{\text {th }}$ century. With ongoing climate and land use change, precipitation might be affected in the future. Deforestation can affect the runoff and increases erosion in mountainous parts of the catchment, even if the annual precipitation does not change. 


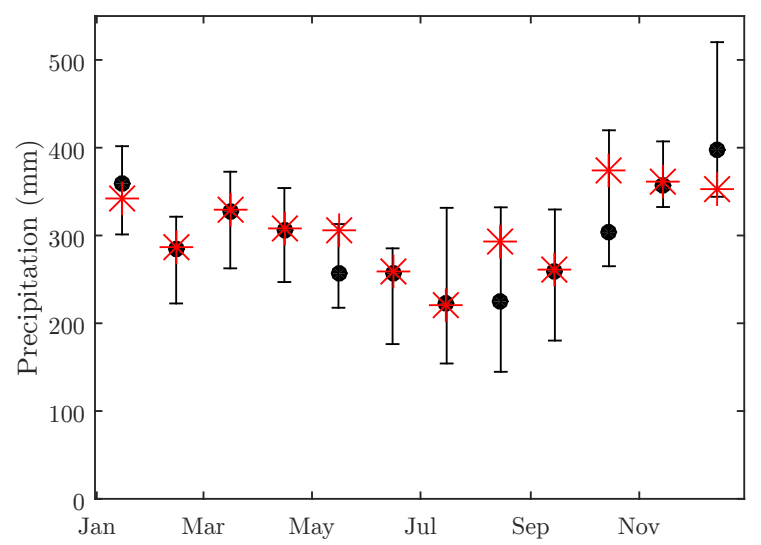

Figure 8.1: Monthly precipitation in the Kapuas catchment upstream of Sanggau (black), median of the years 1998-2015, upper and lower bar extend to $25^{\text {th }}$ and $75^{\text {th }}$ percentile, respectively, estimated from TRMM data (Hidayat et al., 2017), Monthly precipitation in Sintang (1879-1899) (Enthoven, 1903)

This highlights the need for continuous measurements throughout decades.

\subsection{Propagation of river-tides}

Chapter 4 of this thesis highlights the relevance of bed slope for the propagation of the tide, in particular during low flow. Although field measurements and numerical models can give detailed insight into the current state and future evolution of deltas, they alone fall short to explain why this is the case. Chapter 4 provides a simple yet comprehensive model that describes the propagation of tides along a river where a sloping bed and varying discharge result in a varying backwater effect. The importance of the variable backwater for the propagation of tides has recently caught attention (Alebregtse and de Swart, 2016; Cai et al., 2016, 2018).

This is relevant to project the future of tides and mean water levels near the coast (Pelling et al., 2013) and in deltas (Wassmann et al., 2004). Particularly, as deltas are under pressure due to local and global environmental change such as eustatic sea level rise and subsidence (Syvitski et al., 2009), of which of the latter in particular affects Indonesian cities (Chaussard et al., 2013).

\subsection{Acoustic monitoring of suspended sediment transport}

Discharge and sediment transport of many rivers of the world remains ungauged, partly because direct sampling of sediment on a large scale and over a longer period of time requires more resources. Surrogate measurements 
with acoustic instruments can be an affordable alternative. Chapter 5 shows that VADCP backscatter can reveal concentrations and transport of suspended sand in the Kapuas River and improves existing calibration methods to determine the sand fraction in presence of silt and clay. Although this reveals the suspended sand which is most relevant for the bed morphology, fine silt and clay remain invisible to the ADCP. The improved method requires, as before, an extensive calibration with reference samples. State of the art methods still opportunistically exploit the single frequency backscatter measured as a by-product of velocity-metres. Future instrumentation should introduce multifrequency acoustics to increase the reliability of suspended sediment with a wide size range without requiring field site-specific calibrations.

\subsubsection{Sediment concentration and load of rivers on Borneo}

Rivers on Borneo are globally the second largest source of sediment delivered to the sea, only surpassed by those on Papua-New Guinea (Milliman et al., 1999). Despite this, the sediment concentration determined from the water samples during the work on this thesis is low (chapter 5). WBMsed predicts cross-sectional-averaged total concentrations of $210-222 \mathrm{~g} / \mathrm{m}^{3}$ for discharges of $3100-3850 \mathrm{~m}^{3} / \mathrm{s}$, which are equivalent to those during the bifurcation measurements. Hence, modelled concentrations are more than four times as large as the sampled concentrations. The sampled concentrations are comparable in magnitude to those observed in the Berau (Buschman et al., 2012) and Mahakam rivers (Sassi et al., 2013), which are both located in East Kalimantan. The sampled concentration is much lower than that observed in the Rajang river in Sarawak (Staub and Gastaldo, 2000), in the north-western part of the island. The particle size distribution of the suspended sediment in the Kapuas differs from that of the Mahakam. The Mahakam drains the neighbouring catchment of comparable size and similar hydrology (Hidayat et al., 2017). The mean size of the sediment measured by us with the LISST is only half as large as that observed in the Mahakam delta (Sassi et al., 2013). Furthermore, the suspended sediment in the Mahakam does not contain a fine fraction, that remains in suspension during slack water (Sassi et al., 2013). These differences might be due to seasonal variation or recent logging activities, or of fine sediment in the Mahakam lake area. The Mahakam also has a larger slope than the Kapuas (Sibinga, 1953).

Several factors can contribute to the low sampled sediment concentrations. First, during the bifurcation measurements, the river discharge was only about $40 \%$ of the maximum observed in the same year. However, this cannot ex- 
plain the differences to the WBMsed concentrations, as those are already predicted for the low discharges. Second, high altitudes in Kalimantan retain an intact forested cover, while high altitudes in Sarawak have already lost a substantial part of it (Gaveau et al., 2016). Third, Milliman et al. (1999), Cohen et al. (2013) and Syvitski et al. (2014) calibrated their models with data from small catchments on Java that have easily erodible volcanic deposits and large slopes. The topography and geology of relatively large river basins on Borneo are very different (Hall and Nichols, 2002). Although models can give a rough overview of the sediment delivery, sediment yields amongst rivers in the Indonesian Archipelago are probably more variable than predicted. The total sediment yield of Borneo will remain uncertain until its major rivers have been systematically surveyed and monitored.

\subsection{Bifurcations}

Bifurcation dynamics are relevant for managed waterways, such as those in The Netherlands. Long-term morphological stability is desired to maintain the distributary channel network at a minimal cost. The recent replacements of groins by longitudinal training dams has led to an entirely new application in this regard (Le et al., 2018). Short-term stability is also relevant, as a slight shift of the discharge division during the passage of a flood wave can already lead to overtopping of dykes (Schielen et al., 2007).

Conceptual and idealized models give insight into the morphodynamic of river bifurcations. Chapter 6 shows that the division of sediment at the Kapuas bifurcations is at a first glance consistent with the theory. A relatively large fraction of sediment is diverted to side branches in straight reaches, whereas a relatively small fraction of sediment is diverted to side branches in outer bends. Yet, the survey of the Kapuas river also reveals that existing models are incomplete. Bend flow controls the sediment division in the Kapuas through bed material sorting, and wide inlets of the Kapuas side branches reduce the amount of diverted sediment. Bed material heterogeneity is typically neglected even in detailed numerical models (Kleinhans et al., 2008; Le et al., 2018), and state of the art modelling suites are not even capable of modelling planform changes. Chapter 7 provides a thorough mathematical analysis of the sediment division at an idealized bifurcation, which confirms the role of the inlet width and shows that existing models do not properly scale up to dimensions of rivers. 


\subsection{Future of the Kapuas River and its Delta}

Sea level rise poses a major threat to global deltas, in particular those without sea defences, like that of the Kapuas (Nicholls and Cazenave, 2010). A simple projection based a probable sea level rise of $0.5 \mathrm{~m}$ until 2100 (Church et al., 2013) and a land surface slope of $510^{-5}$, yields that the shoreline of West Kalimantan is likely to retreat at least by $10 \mathrm{~km}$ throughout the $21^{\text {st }}$ century. This is only approximately $1 / 10^{\text {th }}$ an of the total area of the alluvial plain donstream of Tayan. The exact retreat depends on how far acretion of coastal mangrove forests can keep up with sea level rise (Woodroffe, 1990). However, much more land could be lost to the sea due to subsidence, as drained peat land can sink ten times as rapid as then the current rate of sea level rise (Hooijer et al., 2012). Good practices to mitigate the impact of sea level rise are likely the protection of mangroves forests, and to allow deposition by overbank flow, which requires the river not to be dyked and sand mining to be curbed. The largest city of West Kalimantan, Pontianak is located just $20 \mathrm{~km}$ from the coast line, and thus directly at threat. As of 2013, roads already flood when heavy local precipitation coincides with high tide. Such floods are likely to become more frequent. Damage could be minimized by encouraging locals to to elevate the ground floor of new houses. At the moment, even upmarked houses are built on ground level, with the consequence of the ground floor being flooded, like the house in Pontianak occupied by the author in 2014. Another challenge is the shortage of drinking water during the dry season (Deynoot, 2011), which will probably require the relocation of the current water intake farther up the Landak river.

\subsection{Final Thoughts}

As of 2018, Indonesia's population and land surface area measure approximately half of those of the European Union, but it's gross domestic product amounts only to $6 \%$. Indonesia thus has the potential to rise to one of the leading economic powers throughout the $21^{\text {st }}$ century. As a country that consists of an archipelago, coastal and estuarine areas will play an important role in this development. The protection of environmental services and mitigation of climate change are essential to Indonesia's long term success (Delinom, 2008; Wise et al., 2016; van Oudenhoven et al., 2015).

This also holds for West Kalimantan, where the provincial capital of Pontianak is situated merely $20 \mathrm{~km}$ from the coast, so that the ground floors of houses are flooded when strong local precipitation coincides with high tides, 
and the fresh water supply occasionally fails during the dry season (Deynoot, 2011). While these inconveniences can be ameliorated with simple construction measures, it is less clear how the Kapuas delta channel network will respond to local and global environmental change.

Detailed numerical modelling may shed more light onto future scenarios. However, ongoing work by the author shows that it is non-trivial, if not even infeasible, to reliably reproduce the complex morphodynamic processes of the Kapuas River bifurcations with state-of-the-art modelling suites. Empirical knowledge is thus essential for the projection of future scenarios. However, so far we know little about the recent Holocene geologic history of the Kapuas meander belts and lowland channel networks. This gap needs to be closed by a thorough survey of the strata of scroll bars, derelict channels, as well as of active bifurcations. 
0 


\section{Summary}

Dynamic and complex, delta channels networks are fascinating theatres of hydrodynamics and morphology. Once a river reaches the delta plain, it bifurcates, changes its course, and forms channel patterns that are unlike those found further upstream. Tides influence and enrich the channel patterns near the sea. Along the delta plain fluvial channel patterns give way to those of the tides, but not much is known about the transition that connects the fluvially and tidally dominated reaches. The motivation for this thesis is to study the fluvial-tidal transition along the Kapuas River. The Kapuas River has been constrained neither by groins nor by banks so far, which makes it ideal to study hydrodynamic and morpodynamic processes in a pristine setting. The core of this thesis consists of six chapters that each address one central objective regarding the fluvial-tidal transition. These are summarized in the following paragraphs.

River discharge is an integral quantity in the hydrological cycle and a driver of sediment transport. Reliable monitoring of river discharge is therefore essential to understand river physiography and to properly manage deltas. Discharge is conventionally inferred from water levels. This approach fails where the relation between velocity and stage is not unique, which is the case in tidally influenced and backwater affected reaches. Direct measurement of the flow velocity can overcome this ambiguity. Chapter 2 explores the suitability of HADCPs for this task. An HADCP can cover only a part of a cross-section when a river is large so that the measurement has to be upscaled to obtain the discharge. The relation between the measured velocity and the total discharge is complicated as the velocity distribution within the river cross-section varies with the stage. This especially deteriorates measurements near the bank, where HADCPs are typically deployed. Chapter 2 introduces a thorough statistical analysis of this uncertainty. Field data from two field sites show, that the 
HADCP does not considerably improve the accuracy at the upstream gauging station, where the stage-discharge relation is reliable, whereas it is indispensable for monitoring the discharge at the tidally affected gauging station close to the sea.

Both rivers and estuaries show distinct downstream trends of cross-section geometry and bed material characteristics. However, the trends of the crosssection geometry along the channels, as well as the importance of the various channel forming processes differ between rivers and estuaries. While the hydrodynamics and morphology of rivers and estuaries are individually well understood, little is known about the fluvial-tidal transition. The same holds for the differences and similarities of concurrent distributary channels in delta channel networks. Chapter 3 sheds more light on the morphology of the fluvial-tidal transition along the distributaries of the Kapuas River.

The Kapuas River delta is of mixed fluvial and tidal character and has so far experienced little direct human interference. It is thus ideal to study the fluvial to tidal transition along distributaries. The Kapuas river has one main channel and three small distributaries that branch off along the coastal plain. All distributaries consist of a long fluvial reach that does not converge, i.e. does not widen towards the sea. Each of those terminates in a short tidal funnel which is separated from the sea by a shallow mouth bar. Tidal creeks connect to the distributaries in the South of the delta. The depths of all distributaries decrease in the downstream direction and reach a maximum at the upstream end of the tidal funnels. The main stem of the river also decreases in width towards the sea, until it reaches the tidal funnel. Along the tidal funnels, the width increases and the depth decreases towards the sea. The along-channel trends of the hydraulic geometry thus clearly break between the fluvially and tidally dominated reaches, while the trends are similar amongst the distributaries. This is contrasted by the along-channel trends of the bed material. The bed material grain size decreases along the main distributary both through the fluvial reach and the tidal funnel. There is no break at the transition. In contrast, bed material grain size is discontinuous at the inlets to the side branches. It is coarser in one of the branches and finer in the other. Most notably, the grain size of the side branches increases towards the sea, in contrast to the main river, along which the bed-material grain size decreases. Downstream coarsening in the side branches indicates an undersupply of sediment and increasing tidal influence towards the sea. The discordance between the trends of hydraulic geometry and bed material reveals the difference between deltaic and fluvial morphology.

Idealized models depict tidal rivers as long channels of constant width and 
depth. As a tidal wave propagates along such a river, its range decreases exponentially with increasing distance from the sea. The range decreases more rapidly for stronger river flow. However, observations reveal that along the Kapuas River, tides propagate remarkably differently (chapter 4 ). The propagation of tides up the Kapuas river can only be understood when the seasonal backwater dynamics are considered. The tidally averaged water depth remains only constant along the Kapuas river during normal flow. During low flow, the depth gradually decreases in the upstream direction. This depth convergence compensates the frictional damping in the downstream part of the tidal river so that the tidal range hardly decreases. Shallow depths intensify friction in the upstream part of the tidal river so that the tidal range decreases more rapidly. This leads to a pronounced knick-point of the tidal range along the river, and effectively limits the propagation of tides beyond the point where the bed reaches sea level. Further analysis shows that this also applies to the overtide and subtidal harmonics. The extended model presented in chapter 4 leads to a better understanding of tidal river dynamics, as the water levels in deltas are in general subject to seasonally varying backwater.

Acoustics allow for measurements of suspended sediment with high spatial and temporal resolution with little effort and cost. However, so far, no dedicated acoustic instruments for the measurement of suspended sediment are available. One has to rely on acoustic backscatter that is recorded by ADCPs as a by-product of the velocity. Acoustic backscatter is sensitive both to grain size and sediment concentration. Changes thereof can only be sensed with multi-frequency backscatter. Sediment concentrations determined from mono-frequency ADCP backscatter are ambiguous, as ADCPs operate only at a single frequency. Furthermore, a specific calibration is required for each field site and flood stage. Conventionally, the total concentration of the suspended sediment is determined from the backscatter. However, fine particles, such as silt and clay, become de-facto imperceptible to the ADCP when coarser particles, such sand, are in suspension. Chapter 5 introduces a novel method that infers only the coarse fraction of the suspended sediment as well as a robust uncertainty analysis by means of bootstrapping. The improved method is straightforward to apply and better fits the data collected in the Kapuas than the conventional method.

The division of sediment between delta channel branches is sensitive to local processes at the bifurcations, and thus much more complicated than the division of discharge. The morphodynamics of bifurcation in large sand bed rivers is not fully understood, which is largely due to the limited number of field studies on this topic. Chapter 6 presents measurements of flow and sedi- 
ment division at two strongly asymmetric bifurcations of the Kapuas River. At both bifurcations, a smaller distributary channel branches off to the side and turns around a spit. The planform asymmetry is reflected in the bed geometry, where the bed slopes down across the approaching channel towards the side channel. The bed level slightly rises at the bifurcation and drops again into a scour hole, which extends into the side branch. This renders conventional bifurcation models that assume the bed to slope up towards the smaller branch, or even a step of the bed level, not applicable. The measurements show that the transported sediment partly consists of silt and clay that remains in suspension over the tidal cycle, and partly of sand that settles and resuspends with the tide. The differences between water and sediment division ratios between the two bifurcations subject to study confirm that the particular location along meander bend strongly influences the division. The bulk of the suspended sand bypasses the side branch that branches off in an outer bend, whereas a relatively large fraction of suspended sand enters the side branch that branches off in a nearly straight reach. In contrast to the conventional models, observed patterns of the suspended sediment concentrations indicate that the division of sediment is controlled by sorting of the bed material rather than directly by the secondary flow. Diversion of sediment seems to be reduced by the comparatively large inlet width and area of the side branches at the bifurcations. Local bank migration thus seems to stabilize and not to destabilize the side branches, as previously assumed.

Many river bifurcations, like those of the Kapuas, are strongly asymmetric so that the branches are of different size. Such a configuration is similar to engineered lateral diversions, which have been extensively studied. At such a bifurcation, the secondary flow diverts more water from the lower part than from the higher part of the water column into the side branch, which is referred to as the Bulle effect. This causes a larger fraction of sediment than water to be diverted, as the sediment concentration is higher near the bed than near the surface. Artificial side branches therefore rapidly fill in. However, side branches in rivers are often surprisingly stable, which indicates that Bulle's effect is compensated for by other factors. Measurements presented in chapter 6 indicate that an enlarged inlet to the side branch may reduce the fraction of the diverted sediment. Several ad-hoc and semi-empirical models of the sediment division exist that are based on laboratory experiments. However, none is based on a proper mathematical analysis of the dividing flow at lateral diversions at the river scale. Chapter 7 presents a thorough mathematical analysis of an idealized bifurcation based on potential flow. It reveals that the flow depends primarily on a single parameter, which is the relative 
strength of the approaching flow relatively to the diverted flow. The sediment division is uniquely determined in combination with a second parameter, which scales with depth. The mathematical analysis confirms the hypothesis that less sediment is diverted to wider inlets. It also reveals that existing empirical models do not scale consistently, and thus do not necessarily apply to bifurcations in natural rivers. 
0 


\section{Acknowledgements}

A large project like the Kapuas expedition is not just the work of a single man, and so I am indebted to all the persons who contributed their efforts to make this project a success.

I thank our Indonesian project members for inviting and welcoming me and the crew to Indonesia: Robert Delinom (LIPI) for issuing the invitation; Ibu Nining (IT Bandung) for supporting the proposal; Pak Hidayat (LIPI Cibinong) for arranging a good boat in Rasau and sieving in Cibinong; Siti Aisyah (LIPI Cibinong) for sieving bottom samples; Pak Gusti (UNTAN Pontianak) for supporting the proposal, arranging lodging in Pontianak, as well as analysing water samples in his laboratory; Pak Pramulja (UNTAN Pontianak) for establishing contacts with locals and helping in the field; as well as Pak Buyian (Rasau Jaya) for providing his boat for measurements.

I thank my supervisor Ton Hoitink who wrote the proposal and won the grant before I joined the project, for providing me with the unique opportunity to lead the expedition to the Kapuas River, and for guiding me thereafter through the publication process. I also thank my promotor Remko Uijlenhoet for his support. I thank my co-authors for their contributions, especially Paul Torfs as well as Eric Deleersnijder, the latter also for his support of the project proposal.

I thank Bart Vermeulen, for showing me how to use ADCPs, and for helping with the deployment and removal of the instruments at the start and end of the survey. I thank Tjitske Geertsema, for joining me for seven months in Indonesia and for participating in the measurements. I thank Maximiliano Sassi, who undertook a previous expedition to the Mahakam River as well as a pilot survey to the Kapuas, and on whose experience the expedition to the Kapuas River largely relied. I thank Rolf Aalto for joining the kick-off campaign. I 
thank Chris Kaldenhoff for training me as a diver. I thank Pieter Hazenberg for helping with the preparation of the equipment for the field campaign.

I thank the students who joined in the field and assisted with the measurements: Anne-Kees Huisman, Judith Snethlage, Laura Schlebes, Mochamed Februiatno, Noud Kuilder, Reza Ghatayu, Zebulon Spruijt; as well as the students who did their thesis within the framework of the Kapuas project without having had the opportunity to work in the field: David Muñoz Pauta, Kees Teuling, Rob van Beuken, Timo Kelder, Yannick Mijnheer. I wish all students the best for their future.

I thank those companies and institutes in the Netherlands who lend instruments for the expedition free of charge: Jelle Molenaar at Deltares for a LISST, Constantijn Kappelhof at Rijkswaterstaat for a vertical ADCP, Sven Ober at the Royal Netherlands Institute for Sea Research (NIOZ) for a Niskin Bottle, Bert Speelman at Rijkswaterstaat for a trap sampler. I thank Willem Ottevanger at Deltares for giving advice on set up of several Delft 3D and Delft Flexible mesh models. I thank Sikko Visscher at Royal Netherlands Academy of Arts and Sciences (KNAW) for supporting the visa application.

I thank the USGS and NASA for making satellite data of the Landsat and TRMM missions easily accessible free of charge. I thank the Koninklijk Instituut voor de Tropen (KIT) and Leiden University as well as the Perry-Castañeda Library and the David Rumsey Map collection for making their historical map collection online accessible free of charge. I also thank the GNU/Linux community for providing a free and reliable operating system and programmes with which this thesis was created.

Finally, I thank the Royal Netherlands Academy of Arts and Sciences (KNAW) for funding this project within the framework of Scientific Programme Indonesia - Netherlands (SPIN). 


\section{Bibliography}

Abam, T. K. S., Impact of dams on the hydrology of the Niger Delta, Bulletin of Engineering Geology and the Environment, 57(3), 239-251, 1999.

Ainslie, M. A., and J. G. McColm, A simplified formula for viscous and chemical absorption in sea water, The Journal of the Acoustical Society of America, 103(3), 1671-1672, 1998.

Alebregtse, N. C., and H. E. de Swart, Effect of river discharge and geometry on tides and net water transport in an estuarine network, an idealized model applied to the Yangtze Estuary, Continental Shelf Research, 123, 29-49, 2016.

Allen, J. R. L., Reaction, relaxation and lag in natural sedimentary systems: general principles, examples and lessons, Earth-Science Reviews, 10(4), 263-342, 1974.

Allen, J. R. L., Mud drapes in sand-wave deposits: a physical model with application to the Folkestone Beds (early Cretaceous, southeast England), Philosophical Transactions of the Royal Society of London A, 306(1493), 291-345, 1982.

Allison, M. A., M. T. Ramirez, and E. A. Meselhe, Diversion of Mississippi River water downstream of New Orleans, Louisiana, USA to maximize sediment capture and ameliorate coastal land loss, Water Resources Management, 28(12), 4113-4126, 2014.

American Society for Testing and Materials E11, Standard specification for woven wire test sieve cloth and test sieves, 2009.

Anderson, V. C., Sound scattering from a fluid sphere, The Journal of the Acoustical Society of America, 22(4), 426-431, 1950.

Andren, H., Development of the Laitaure delta, Swedish Lappland: A. Study of Growth, Dis- tributary Forms, and Processes, Ph.D. thesis, Uppsala University, Uppsala, Sweden, 1994.

Anshari, G. Z., M. Afifudin, M. Nuriman, E. Gusmayanti, L. Arianie, R. Susana, R. W. Nusantara, J. Sugardjito, and A. Rafiastanto, Drainage and land use impacts on changes in selected peat properties and peat degradation in West Kalimantan Province, Indonesia, Biogeosciences, 7(11), 3403, 2010.

Anthony, E. J., G. Brunier, M. Besset, M. Goichot, P. Dussouillez, and V. L. Nguyen, Linking rapid erosion of the Mekong River delta to human activities, Scientific Reports, 5, 14,745, 2015.

Apel, H., A. H. Thieken, B. Merz, and G. Blöschl, Flood risk assessment and associated uncertainty, Natural Hazards and Earth System Science, 4(2), 295-308, 2004.

Arce, G. R., Y.-T. Kim, and K. E. Barner, Orderstatistic filtering and smoothing of time-series Part I, in Order Statistics: Applications, Handbook of Statistics, vol. 17, pp. 525-554, Elsevier, Amsterdam, The Netherlands, 1998.

Asselman, N. E. M., Fitting and interpretation of sediment rating curves, Journal of Hydrology, 234(3), 228-248, 2000.

Axelsson, V., The Laitaure Delta: A. study of deltaic morphology and processes, Geografiska Annaler. Series A., Physical Geography, 49(1), 1-127, 1967.

Aǵaçcioǵlu, H., and F. Önen, Clear-water scour at a side-weir intersection along the bend, Irrigation and Drainage, 54(5), 553-569, 2005.

Bakker, K., Privatizing water: governance failure and the world's urban water crisis, Cornell University Press, 2010. 
Berne, S., P. Castaing, E. Le Drezen, and G. Lericolais, Morphology, internal structure, and reversal of asymmetry of large subtidal dunes in the entrance to Gironde Estuary (France), Journal of Sedimentary Research, 63(5), 1993.

Bertoldi, W., and M. Tubino, River bifurcations: Experimental observations on equilibrium configurations, Water Resources Research, 43(10), 2007.

Beven, K. J., Rainfall-runoff modelling: the primer, John Wiley \& Sons, 2011.

Blanckaert, K., and H. J. De Vriend, Nonlinear modeling of mean flow redistribution in curved open channels, Water Resources Research, 39(12), 2003.

Blanckaert, K., and H. J. De Vriend, Secondary flow in sharp open-channel bends, Journal of Fluid Mechanics, 498, 353-380, 2004.

Blight, B. J. N., Sampling from an autocorrelated finite population, Biometrika, 60(2), 375-385, 1973.

Blom, A., E. Viparelli, and V. Chavarrías, The graded alluvial river: Profile concavity and downstream fining, Geophysical Research Letters, 43(12), 6285-6293, 2016.

Blom, A., L. Arkesteijn, V. Chavarrías, and E. Viparelli, The equilibrium alluvial river under variable flow and its channel-forming discharge, Journal of Geophysical Research: Earth Surface, 122(10), 1924-1948, 2017.

Bloom, D. E., D. Canning, and P. N. Malaney, Population dynamics and economic growth in Asia, Population and Development Review, 26, 257290, 2000.

Böhme, M., M. Aiglstorfer, D. Uhl, and O. Kullmer, The antiquity of the Rhine River: stratigraphic coverage of the Dinotheriensande (Eppelsheim Formation) of the Mainz Basin (Germany), PLoS One, 7(5), e36,817, 2012.

Boiten, W., Hydrometry, IHE Delft lecture note series, Balkema, A. A., Amsterdam, The Netherlands, 2000.

Bol, D. C. C., The influence of the upper Kapuas wetland area on the Kapuas flow characteristics, West-Kalimantan, Indonesia, 2015.

Bolla Pittaluga, M., R. Repetto, and M. Tubino, Channel bifurcation in braided rivers: equilibrium configurations and stability, Water Resources Research, 39(3), 2003.

Bolla Pittaluga, M., R. Luchi, and G. Seminara, On the equilibrium profile of river beds, Journal of Geophysical Research: Earth Surface, 119(2), 317-332, 2014.

Bolla Pittaluga, M., G. Coco, and M. G. Kleinhans, A unified framework for stability of channel bifurcations in gravel and sand fluvial systems, Geophysical Research Letters, 42(18), 7521-7536, 2015a.

Bolla Pittaluga, M., N. Tambroni, A. Canestrelli, R. Slingerland, S. Lanzoni, and G. Seminara, Where river and tide meet: The morphodynamic equilibrium of alluvial estuaries, Journal of Geophysical Research: Earth Surface, 120(1), 7594, 2015b.

Boyd, R., R. Dalrymple, and B. A. Zaitlin, Classification of clastic coastal depositional environments, Sedimentary Geology, 80(3-4), 139-150, 1992.

Bradley, S., Horizontal ADCP for remote mapping of currents, in Proceedings of the IEEE Sixth Working Conference on Current Measurement, 1999, pp. 103-107, IEEE, Piscataway, NJ, 1999.

Brakenridge, G., J. Syvitski, E. Niebuhr, I. Overeem, S. Higgins, A. Kettner, and L. Prades, Design with nature: Causation and avoidance of catastrophic flooding, Myanmar, Earth-Science Reviews, 165, 81-109, 2017.

Brooks, D. A., The hydrokinetic power resource in a tidal estuary: The Kennebec River of the central Maine coast, Renewable Energy, 36(5), 14921501, 2011.

Brunier, G., E. J. Anthony, M. Goichot, M. Provansal, and P. Dussouillez, Recent morphological changes in the Mekong and Bassac river channels, Mekong delta: the marked impact of river-bed mining and implications for delta destabilisation, Geomorphology, 224, 177-191, 2014.

Budiyono, Y., J. Aerts, J. Brinkman, M. A. Marfai, and P. Ward, Flood risk assessment for delta mega-cities: a case study of Jakarta, Natural Hazards, 75(1), 389-413, 2015.

Bulle, H., Untersuchungen über die Geschiebeableitung bei der Spaltung von Wasserläufen: Modellversuche aus dem Flussbaulaboratorium der Technischen Hochschule zu Karlsruhe, VDIVerlag, 1926.

Bunt, J. A. C., P. Larcombe, and C. F. Jago, Quantifying the response of optical backscatter devices and transmissometers to variations in sus- 
pended particulate matter, Continental Shelf Research, 19(9), 1199-1220, 1999.

Buschman, F. A., Flow and sediment transport in an Indonesian tidal network, Ph. D. Thesis, Utrecht University, Utrecht, 2011.

Buschman, F. A., A. J. F. Hoitink, M. Van der Vegt, and P. Hoekstra, Subtidal flow division at a shallow tidal junction, Water Resources Research, 46(12), 2010.

Buschman, F. A., A. J. F. Hoitink, S. M. de Jong, P. Hoekstra, H. Hidayat, and M. G. Sassi, Suspended sediment load in the tidal zone of an Indonesian river, Hydrology and Earth System Sciences, 16(11), 4191-4204, 2012.

Buschman, F. A., A. J. F. Hoitink, M. van der Vegt, and P. Hoekstra, Subtidal water level variation controlled by river flow and tides, Water $R e$ sources Research, 45(10), 2009.

Buschman, F. A., M. van der Vegt, A. J. F. Hoitink, and P. Hoekstra, Water and suspended sediment division at a stratified tidal junction, Journal of Geophysical Research: Oceans, 118(3), 14591472, 2013.

Cai, H., H. H. G. Savenije, and M. Toffolon, Linking the river to the estuary: influence of river discharge on tidal damping, Hydrology and Earth System Sciences, 18(1), 287-304, 2014.

Cai, H., H. H. G. Savenije, C. Jiang, L. Zhao, and Q. Yang, Analytical approach for determining the mean water level profile in an estuary with substantial fresh water discharge, Hydrology and Earth System Sciences, 20(3), 1177-1195, 2016.

Cai, H., Q. Yang, Z. Zhang, X. Guo, F. Liu, and S. Ou, Impact of river-tide dynamics on the temporalspatial distribution of residual water level in the pearl river channel networks, Estuaries and Coasts, pp. 1-19, 2018.

Canestrelli, A., S. Lanzoni, and S. Fagherazzi, Onedimensional numerical modeling of the longterm morphodynamic evolution of a tidallydominated estuary: The Lower Fly River (Papua New Guinea), Sedimentary Geology, 301, 107119, 2014.

Carling, P., The concept of dominant discharge applied to two gravel-bed streams in relation to channel stability thresholds, Earth Surface Processes and Landforms, 13(4), 355-367, 1988.

Cartwright, D. E., and R. J. Tayler, New computations of the tide-generating potential, Geophysical Journal International, 23(1), 45-73, 1971.
Castro-Orgaz, O., and W. H. Hager, NonHydrostatic Free Surface Flows, Springer, 2017.

Chambers, J. L. C., I. Carter, I. R. Cloke, J. Craig, S. J. Moss, and D. W. Paterson, Thin-skinned and thick-skinned inversion-related thrusting A. structural model for the Kutai Basin, Kalimantan, Indonesia, Thrust Tectonics and Hydrocarbon Systems: AAPG Memoir, 82, 614-634, 2004.

Chatanantavet, P., M. P. Lamb, and J. A. Nittrouer, Backwater controls of avulsion location on deltas, Geophysical Research Letters, 39(1), 2012.

Chaussard, E., F. Amelung, H. Abidin, and S.-H. Hong, Sinking cities in Indonesia: ALOS PALSAR detects rapid subsidence due to groundwater and gas extraction, Remote Sensing of Environment, 128, 150-161, 2013.

Cheng, R. T., J. W. Gartner, J. Mason, R. R., J. E. Costa, W. J. Plant, K. R. Spicer, F. P. Haeni, N. B. Melcher, W. C. Keller, and K. Hayes, Evaluating a Radar-Based, Non Contact Streamflow Measurement System in the San Joaquin River at Vernalis, California, Tech. rep., U. S. Geological Survey, 2015.

Cheng, Z., K. Lee, D. Kim, M. Must, P. Vidmar, and J. Hulme, Experimental Evidence on the Performance of Rating Curves for Continuous Discharge Estimation in Complex Flow Situations, (submitted), Journal of Hydrology, 2018.

Chow, V. T., Open channel flow, London: McGRAWHILL, 11(95), 99-136, 1959.

Church, J. A., P. U. Clark, A. Cazenave, J. M. Gregory, S. Jevrejeva, A. Levermann, M. A. Merrifield, G. A. Milne, R. S. Nerem, P. D. Nunn, A. J. Payne, W. T. Pfeffer, D. Stammer, and A. S. Unnikrishnan, Sea Level Change, in Climate Change 2013: The Physical Science Basis. Contribution of Working Group I to the Fifth Assessment Report of the Intergovernmental Panel on Climate Change, edited by T. F. Stocker, D. Qin, G. K. Plattner, M. Tignor, S. K. Allen, J. Boschung, A. Nauels, Y. Xia, V. Bex, and P. M. Midgley, Cambridge University Press, Cambridge, United Kingdom and New York, NY, USA, 2013.

Cohen, S., A. J. Kettner, J. P. Syvitski, and B. M. Fekete, WBMsed, a distributed global-scale riverine sediment flux model: Model description and validation, Computers \& Geosciences, 53, 80-93, 2013. 
Coleman, J. M., Dynamic changes and processes in the Mississippi River delta, Geological Society of America Bulletin, 100(7), 999-1015, 1988.

Coles, D., The law of the wake in the turbulent boundary layer, Journal of Fluid Mechanics, 1(2), 191-226, 1956.

Constantine, J. A., T. Dunne, H. Piégay, and G. Mathias Kondolf, Controls on the alluviation of oxbow lakes by bed-material load along the Sacramento River, California, Sedimentology, 57(2), 389-407, 2010.

Cooper, J. P., "Fear God; Fear the Bogaze": The Nile Mouths and the Navigational Landscape of the Medieval Nile Delta, Egypt, Al-Masaq, 24(1), 53-73, 2012.

Corwin, A., K. Jarot, I. Lubis, K. Nasution, S. Suparmawo, A. Sumardiati, S. Widodo, S. Nazir, G. Orndorff, Y. Choi, et al., Two years' investigation of epidemic hepatitis E. virus transmission in West Kalimantan (Borneo), Indonesia, Transactions of the Royal Society of Tropical Medicine and Hygiene, 89(3), 262-265, 1995.

Costanza, R., W. J. Mitsch, and J. W. Day Jr, A new vision for New Orleans and the Mississippi delta: applying ecological economics and ecological engineering, Frontiers in Ecology and the Environment, 4(9), 465-472, 2006.

Costard, F., and E. Gautier, The Lena River: Hydromorphodynamic features in a deep permafrost zone, in Large Rivers: Geomorphology and Management, pp. 225-233, John Wiley \& Sons, Ltd, 2007.

Crosato, A., and E. Mosselman, Simple physicsbased predictor for the number of river bars and the transition between meandering and braiding, Water Resources Research, 45(3), 2009.

Cui, B., Q. Yang, Z. Yang, and K. Zhang, Evaluating the ecological performance of wetland restoration in the Yellow River Delta, China, Ecological Engineering, 35(7), 1090-1103, 2009.

Cunge, J. A., F. M. Holly, and A. Verwey, Practical aspects of computational river hydraulics, Monographs and Surveys in Water Resources Engineering 3, Pitman Publishing, Boston,U. S. A, 1980.

Dai, A., and K. E. Trenberth, Estimates of freshwater discharge from continents: Latitudinal and seasonal variations, Journal of Hydrometeorology, 3(6), 660-687, 2002.

D'Alpaos, A., S. Lanzoni, M. Marani, S. Fagherazzi, and A. Rinaldo, Tidal network ontogeny: Chan- nel initiation and early development, Journal of Geophysical Research: Earth Surface, 110(F2), 2005.

D'Alpaos, A., S. Lanzoni, M. Marani, and A. Rinaldo, On the O'Brien-Jarrett-Marchi law, Rendiconti Lincei, 20(3), 225-236, 2009.

Dalrymple, R. W., and K. Choi, Morphologic and facies trends through the fluvial-marine transition in tide-dominated depositional systems: a schematic framework for environmental and sequence-stratigraphic interpretation, EarthScience Reviews, 81(3), 135-174, 2007.

Dalrymple, R. W., D. A. Mackay, A. A. Ichaso, and K. S. Choi, Processes, morphodynamics, and facies of tide-dominated estuaries, in Principles of Tidal Sedimentology, pp. 79-107, Springer, 2012.

Dalrymple, R. W., C. E. Kurcinka, B. V. J. G. Jablonski, A. A. Ichaso, and D. A. Mackay, Deciphering the relative importance of fluvial and tidal processes in the fluvial-marine transition, in Developments in Sedimentology, vol. 68, pp. 3-45, Elsevier, 2015.

Dargahi, B., Three-dimensional flow modelling and sediment transport in the River Klarälven, Earth Surface Processes and Landforms, 29(7), 821852, 2004.

Davies, G., and C. D. Woodroffe, Tidal estuary width convergence: Theory and form in North Australian estuaries, Earth Surf. Proc. Land., 35(7), 737-749, 2010.

Davis Jr, R. A., and R. W. Dalrymple, Principles of tidal sedimentology, Springer Science \& Business Media, 2011.

Day, J. W., D. F. Boesch, E. J. Clairain, G. P. Kemp, S. B. Laska, W. J. Mitsch, K. Orth, H. Mashriqui, D. J. Reed, L. Shabman, et al., Restoration of the Mississippi Delta: lessons from hurricanes Katrina and Rita, Science, 315(5819), 1679-1684, 2007.

De Heer, A., and E. Mosselman, Flow structure and bedload distribution at alluvial diversions, in River Flow, vol. 2004, pp. 801-806, 2004.

De Vriend, H. J., A mathematical model of steady flow in curved shallow channels, Journal of Hydraulic Research, 15(1), 37-54, 1977.

De Vriend, H. J., Velocity redistribution in curved rectangular channels, Journal of Fluid Mechanics, 107, 423-439, 1981.

De Vriend, H. J., M. van Koningsveld, S. G. J. 
Aarninkhof, M. B. de Vries, and M. J. Baptist, Sustainable hydraulic engineering through building with nature, Journal of HydroEnvironment Research, 9(2), 159-171, 2015.

Deines, K. L., Backscatter estimation using Broadband acoustic Doppler current profilers, in Current Measurement, 1999. Proceedings of the IEEE Sixth Working Conference on, pp. 249253, 1999.

Delinom, R. M., Groundwater management issues in the greater jakarta area, indonesia, TERC Bull, University of Tsukuba, 8(2), 40-54, 2008.

Deynoot, F. J. C. G., Analytical modeling of Salt Intrusion in the Kapuas Estuary, master thesis, 2011.

Di Baldassarre, G., and A. Montanari, Uncertainty in river discharge observations: a quantitative analysis, Hydrology and Earth System Sciences, 13(6), 913-921, 2009.

Döll, P., and S. Siebert, Global modeling of irrigation water requirements, Water Resources Research, 38(4), 2002.

Doodson, A. T., The harmonic development of the tide-generating potential, Proceedings of the Royal Society A, 100(704), 305-329, 1921a.

Doodson, A. T., The harmonic development of the tide-generating potential, Proceedings of the Royal Society A, 100(704), 305-329, 1921b.

Dottori, F., M. L. V. Martina, and E. Todini, A dynamic rating curve approach to indirect discharge measurement, Hydrology and Earth System Sciences, 13(6), 847-863, 2009.

Douglas, I., The impact of land-use changes, especially logging, shifting cultivation, mining and urbanization on sediment yields in humid tropical Southeast Asia: a review with special reference to Borneo, IAHS Publications-Series of Proceedings and Reports-Intern Assoc Hydrological Sciences, 236, 463-472, 1996.

Douglas, I., K. Bidin, G. Balamurugan, N. A. Chappell, R. P. D. Walsh, T. Greer, and W. Sinun, The role of extreme events in the impacts of selective tropical forestry on erosion during harvesting and recovery phases at Danum Valley, Sabah, in Changes And Disturbance In Tropical Rainforest In South-East Asia, pp. 25-37, World Scientific, 1999.

Dronkers, J. J., Tidal computations in rivers and coastal waters, North-Holland Publishing Company, Amsterdam, 1964.
Dutta, S., D. Wang, P. Tassi, and M. H. Garcia, Three-dimensional numerical modeling of the Bulle effect: the nonlinear distribution of nearbed sediment at fluvial diversions, Earth Surface Processes and Landforms, 42(14), 23222337, 2017.

Eaton, B., R. G. Millar, and S. Davidson, Channel patterns: Braided, anabranching, and singlethread, Geomorphology, 120(3-4), 353-364, 2010.

Eaton, B. C., Hydraulic geometry: empirical investigations and theoretical approaches, in Treatise on Geomorphology, edited by J. F. Shroder and E. Wohl, chap. 9, pp. 313-329, Academic Press, San Diego, 2013.

Edmonds, D., R. Slingerland, J. Best, D. Parsons, and N. Smith, Response of river-dominated delta channel networks to permanent changes in river discharge, Geophysical Research Letters, 37(12), 2010.

Edmonds, D. A., Stability of backwater-influenced river bifurcations: A study of the MississippiAtchafalaya system, Geophysical Research Letters, 39(8), 2012.

Edmonds, D. A., and R. L. Slingerland, Mechanics of river mouth bar formation: Implications for the morphodynamics of delta distributary networks, Journal of Geophysical Research: Earth Surface, 112(F2), 2007.

Edmonds, D. A., and R. L. Slingerland, Stability of delta distributary networks and their bifurcations, Water Resources Research, 44(9), 2008.

Eelkema, M., Z. B. Wang, and M. J. F. Stive, Historical morphological development of the Eastern Scheldt tidal basin (The Netherlands), in Proceedings Of Coastal Dynamics 2009: Impacts of Human Activities on Dynamic Coastal Processes (With CD-ROM), pp. 1-11, World Scientific, 2009.

Egbert, G. D., and S. Y. Erofeeva, Efficient Inverse Modeling of Barotropic Ocean Tides, Journal of Atmospheric and Oceanic Technology, 19(2), 183-204, 2002.

Eke, E., G. Parker, and Y. Shimizu, Numerical modeling of erosional and depositional bank processes in migrating river bends with self-formed width: Morphodynamics of bar push and bank pull, Journal of Geophysical Research: Earth Surface, 119(7), 1455-1483, 2014.

Emiroglu, M. E., H. Agaccioglu, and N. Kaya, Discharging capacity of rectangular side weirs in 
straight open channels, Flow Measurement and Instrumentation, 22(4), 319-330, 2011.

Emmerton, C. A., L. F. W. Lesack, and P. Marsh, Lake abundance, potential water storage, and habitat distribution in the Mackenzie River Delta, western Canadian Arctic, Water Resources Research, 43(5), 2007.

Ensign, S. H., and G. B. Noe, Tidal extension and sea-level rise: recommendations for a research agenda, Frontiers in Ecology and the Environment, 16(1), 37-43, 2018.

Enthoven, J. J. K., Bijdragen tot de geographie van Borneo's westerafdeeling, EJ Brill, 1903.

Ericson, J. P., C. J. Vörösmarty, S. L. Dingman, L. G. Ward, and M. Meybeck, Effective sea-level rise and deltas: causes of change and human dimension implications, Global and Planetary Change, 50(1-2), 63-82, 2006.

Ethridge, F. G., and W. A. Wescott, Tectonic setting, recognition and hydrocarbon reservoir potential of fan-delta deposits, in Sedimentology of Gravels and Conglomerates - Memoir 10, pp. 217235, CSPG Special Publications, 1984.

Exner, F. M., Über die wechselwirkung zwischen wasser und geschiebe in flüssen, Sitzungsberichte der Kaiserlichen Akademie der Wissenschaften in Wien - mathematischnaturwissenschaftliche Classe/Inhalt, 134(2a), 165-204, 1925.

Fagherazzi, S., Self-organization of tidal deltas, Proceedings of the National Academy of Sciences, 105(48), 18,692-18,695, 2008.

Fagherazzi, S., and D. J. Furbish, On the shape and widening of salt marsh creeks, Journal of Geophysical Research: Oceans, 106(C1), 991-1003, 2001.

Fagherazzi, S., D. A. Edmonds, W. Nardin, N. Leonardi, A. Canestrelli, F. Falcini, D. J. Jerolmack, G. Mariotti, J. C. Rowland, and R. L. Slingerland, Dynamics of river mouth deposits, Reviews of Geophysics, 53(3), 642-672, 2015.

Fehn, H., Die Oberflächenformen der Insel Borneo: ein Überlick, Geograph. Ges., 1933.

Ferguson, R. I., D. R. Parsons, S. N. Lane, and R. J. Hardy, Flow in meander bends with recirculation at the inner bank, Water Resources Research, 39(11), 2003.

Fernandes, A. M., T. E. Törnqvist, K. M. Straub, and D. Mohrig, Connecting the backwater hydraulics of coastal rivers to fluvio-deltaic sed- imentology and stratigraphy, Geology, 44(12), 979-982, 2016.

Ferrarin, C., F. Madricardo, F. Rizzetto, W. Mc Kiver, D. Bellafiore, G. Umgiesser, A. Kruss, L. Zaggia, F. Foglini, A. Ceregato, et al., Geomorphology of scour holes at tidal channel confluences, Journal of Geophysical Research: Earth Surface, 2018.

Fong, D. A., S. G. Monismith, M. T. Stacey, and J. R. Burau, Turbulent stresses and secondary currents in a tidal-forced channel with significant curvature and asymmetric bed forms, Journal of Hydraulic Engineering, 135(3), 198-208, 2009.

Foreman, M. G. G., Manual for Tidal Heights Analysis and Prediction, Canada Institute of Ocean Sciences Pacific Marine Science Report, Institute of Ocean Sciences, Patricia Bay, 1996.

Frazier, T. G., N. Wood, B. Yarnal, and D. H. Bauer, Influence of potential sea level rise on societal vulnerability to hurricane storm-surge hazards, Sarasota County, Florida, Applied Geography, 30(4), 490-505, 2010.

Friedrichs, C. T., Barotropic tides in channelized estuaries, in Contemporary Issues in Estuarine Physics, edited by A. Valle-Levinson, pp. 27-61, Cambridge University Press, Cambridge, 2010.

Friedrichs, C. T., and O. S. Madsen, Nonlinear diffusion of the tidal signal in frictionally dominated embayments, Journal of Geophysical Research: Oceans, 97(C4), 5637-5650, 1992.

Frings, R. M., Downstream fining in large sandbed rivers, Earth-Science Reviews, 87(1), 3960, 2008.

Frings, R. M., Sedimentary characteristics of the gravel-sand transition in the River Rhine, Journal of Sedimentary Research, 81(1), 52-63, 2011.

Frings, R. M., and M. G. Kleinhans, Complex variations in sediment transport at three large river bifurcations during discharge waves in the river Rhine, Sedimentology, 55(5), 1145-1171, 2008a.

Frings, R. M., and M. G. Kleinhans, Complex variations in sediment transport at three large river bifurcations during discharge waves in the river Rhine, Sedimentology, 55(5), 1145-1171, $2008 b$.

Fugate, D. C., and C. T. Friedrichs, Determining concentration and fall velocity of estuarine particle populations using ADV, OBS and LISST, Continental Shelf Research, 22(11-13), 1867- 
1886, 2002.

Galloway, W. E., Process framework for describing the morphologic and stratigraphic evolution of deltaic depositional systems, in Deltas: Models for Exploration, pp. 87-98, Houston Geological Society, 1975.

Garel, E., and H. Cai, Effects of Tidal-Forcing Variations on Tidal Properties Along a Narrow Convergent Estuary, Estuaries and Coasts, pp. 1-19, 2018.

Garrett, C., and P. Cummins, The power potential of tidal currents in channels, Proceedings of the Royal Society of London A: Mathematical, Physical and Engineering Sciences, 461(2060), 25632572, 2005.

Gartner, J. W., Estimating suspended solids concentrations from backscatter intensity measured by acoustic Doppler current profiler in San Francisco Bay, California, Marine Geology, 211(3), 169-187, 2004.

Gatto, M., L. Mari, E. Bertuzzo, R. Casagrandi, L. Righetto, I. Rodriguez-Iturbe, and A. Rinaldo, Generalized reproduction numbers and the prediction of patterns in waterborne disease, Proceedings of the National Academy of Sciences, 109(48), 19,703-19,708, 2012.

Gaveau, D. L. A., S. Sloan, E. Molidena, H. Yaen, D. Sheil, N. K. Abram, M. Ancrenaz, R. Nasi, M. Quinones, N. Wielaard, et al., Four decades of forest persistence, clearance and logging on Borneo, PloS One, 9(7), e101,654, 2014.

Gaveau, D. L. A., D. Sheil, M. A. Salim, S. Arjasakusuma, M. Ancrenaz, P. Pacheco, E. Meijaard, et al., Rapid conversions and avoided deforestation: examining four decades of industrial plantation expansion in Borneo, Scientific Reports, 6, 32,017, 2016.

Gaweesh, A., and E. Meselhe, Evaluation of sediment diversion design attributes and their impact on the capture efficiency, Journal of $\mathrm{Hy}$ draulic Engineering, 142(5), 04016,002, 2016.

Geleynse, N., J. E. A. Storms, D.-J. R. Walstra, H. R. A. Jagers, Z. B. Wang, and M. J. F. Stive, Controls on river delta formation: Insights from numerical modelling, Earth and Planetary Science Letters, 302(1), 217-226, 2011.

Gingras, M. K., J. A. MacEachern, and S. E. Dashtgard, The potential of trace fossils as tidal indicators in bays and estuaries, Sedimentary Geology, 279, 97-106, 2012.

Ginsberg, S. S., S. Aliotta, and G. O. Lizasoain, Mor- phodynamics and seismostratigraphy of a deep hole at tidal channel confluence, Geomorphology, 104(3-4), 253-261, 2009.

Gleick, P. H., and M. Heberger, Water conflict chronology, in The World's Water, pp. 175-214, Springer, 2012.

Glenn, E. P., C. Lee, R. Felger, and S. Zengel, Effects of water management on the wetlands of the Colorado River Delta, Mexico, Conservation Biology, 10(4), 1175-1186, 1996.

Godin, G., The tide in rivers, The International Hydrographic Review, 61(2), 1984.

Godin, G., Modification of river tides by the discharge, Journal of Waterway, Port, Coastal, and Ocean Engineering, 111(2), 257-274, 1985.

Godin, G., Compact approximations to the bottom friction term, for the study of tides propagating in channels, Continental Shelf Research, 11(7), 579-589, 1991a.

Godin, G., Frictional effects in river tides, in Tidal Hydrodynamics, vol. 379, p. 402, John Wiley \& Sons, 1991b.

Godin, G., The analysis of tides and currents, in Tidal Hydrodynamics, pp. 675-709, John Wiley \& Sons, New York, 1991c.

Godin, G., The propagation of tides up rivers with special considerations on the upper Saint Lawrence River, Estuarine, Coastal and Shelf Science, 48(3), 307-324, 1999.

Godin, G., and A. Martínez, Numerical experiments to investigate the effects of quadratic friction on the propagation of tides in a channel, Continental Shelf Research, 14(7), 723-748, 1994.

Goodfriend, G. A., and D. J. Stanley, Rapid strandplain accretion in the northeastern Nile Delta in the 9th century $\mathrm{AD}$ and the demise of the port of Pelusium, Geology, 27(2), 147-150, 1999.

Gordon, R. L., Acoustic measurement of river discharge, Journal of Hydraulic Engineering, 115(7), 925-936, 1989.

Granville, P. S., A modified law of the wake for turbulent shear layers, Journal of Fluids Engineering, 98(3), 578-580, 1976.

Gray, J. R., and J. W. Gartner, Technological advances in suspended-sediment surrogate monitoring, Water Resources Research, 45(4), W00D29, 2009.

Green, G., On the motion of waves in a variable canal of small depth and width, Transactions 
of the Cambridge Philosophical Society, 6, 457, 1838.

Guerrero, M., and V. Di Federico, Suspended sediment assessment by combining sound attenuation and backscatter measurements-analytical method and experimental validation, Advances in Water Resources, 113, 167-179, 2018.

Guerrero, M., and A. Lamberti, Flow field and morphology mapping using ADCP and multibeam techniques: Survey in the Po River, Journal of Hydraulic Engineering, 137(12), 1576-1587, 2011.

Guerrero, M., R. Szupiany, and M. Amsler, Comparison of acoustic backscattering techniques for suspended sediments investigation, Flow Measurement and Instrumentation, 22(5), 392-401, 2011.

Guerrero, M., N. Rüther, R. Szupiany, S. Haun, S. Baranya, and F. Latosinski, The acoustic properties of suspended sediment in large rivers: consequences on ADCP methods applicability, Water, 8(1), 13, 2016.

Guerrero, M., N. Rüther, S. Haun, and S. Baranya, A combined use of acoustic and optical devices to investigate suspended sediment in rivers, $A d$ vances in Water Resources, 102, 1-12, 2017.

Guerrero, M., R. N. Szupiany, and F. Latosinski, Multi-frequency acoustics for suspended sediment studies: an application in the Parana River, Journal of Hydraulic Research, 51(6), 696-707, 2013.

Guo, L., M. van der Wegen, D. A. Jay, P. Matte, Z. B. Wang, D. Roelvink, and Q. He, River-tide dynamics: Exploration of nonstationary and nonlinear tidal behavior in the Yangtze River estuary, Journal of Geophysical Research: Oceans, 120(5), 3499-3521, 2015.

Habermaas, F., Geschiebeeinwanderung in werkkanäle und deren verhinderung, Ph.D. thesis, Oldenbourg, 1935.

Hack, J. T., Studies of longitudinal stream profiles in Virginia and Maryland, Tech. Rep. 294-B, U. S. Geological Survey, Washington, 1957.

Hager, W. H., An approximate treatment of flow in branches and bends, Proceedings of the Institution of Mechanical Engineers, Part C: Journal of Mechanical Engineering Science, 198(1), 6369, 1984.

Hall, R., and G. Nichols, Cenozoic sedimentation and tectonics in Borneo: climatic influences on orogenesis, Geological Society, London, Special
Publications, 191(1), 5-22, 2002.

Hamilton, L. S., What are the impacts of Himalayan deforestation on the Ganges-Brahmaputra lowlands and delta? Assumptions and facts, Mountain Research and Development, pp. 256-263, 1987.

Hanes, D. M., On the possibility of single-frequency acoustic measurement of sand and clay concentrations in uniform suspensions, Continental Shelf Research, 46, 64-66, 2012.

Hassenpflug, W. C., Branched channel freestreamlines, Computer Methods in Applied Mechanics and Engineering, 159(3-4), 329-354, 1998.

Haught, D., J. G. Venditti, and S. A. Wright, Calculation of in situ acoustic sediment attenuation using off-the-shelf horizontal A. DCPs in low concentration settings, Water Resources Research, 53(6), 5017-5037, 2017.

Henderson, F. M., Open Channel Flow, Macmillan Series in Civil Engineering, Macmillan, Basingstoke, UK, 1966.

Herawati, H., S. Suripin, S. Suharyanto, and T. Hetwisari, Analysis of river flow regime changes related to water availability on the Kapuas river, Indonesia, Irrigation and Drainage, 2017.

Herbertson, J. G., and Y. R. Fares, Bed topography changes produced by partial cut-off of a meander loop, in Proceedings of the European Conference Advances in Water Resources Technology, pp. 113-120, 1991.

Herrero, A., A. Bateman, and V. Medina, Water flow and sediment transport in a $90^{\circ}$ channel diversion: an experimental study, Journal of Hydraulic Research, 53(2), 253-263, 2015.

Hidayat, H., Runoff, discharge and flood occurrence in a poorly gauged tropical basin: The Mahakam River, Kalimantan, Ph.D. thesis, Wageningen University, Wageningen, The Netherlands, 2013.

Hidayat, H., B. Vermeulen, M. G. Sassi, P. J. J. F. Torfs, and A. J. F. Hoitink, Discharge estimation in a backwater affected meandering river, Hydrology and Earth System Sciences, 15(8), 2717-2728, 2011.

Hidayat, H. H., R. A. J. Teuling, B. Vermeulen, M. Taufik, K. Kästner, T. J. Geertsema, D. C. C. Bol, G. S. Hoekman, H. Haryani, H. A. J. Van Lanen, R. M. Delinom, R. Dijksma, A. G. Z., R. Ningsih, R. Uijlenhoet, and A. J. F. Hoitink, Hydrology of inland tropical lowlands: 
The Kapuas and Mahakam wetlands, Hydrology and Earth System Sciences, 5(21), 2579-2594, 2017.

Hijmans, R., and et al., GADM database of Global Administrative Areas, version 2.0, 2012.

Hill, A. E., and A. J. Souza, Tidal dynamics in channels: 2. Complex channel networks, Journal of Geophysical Research: Oceans, 111(C11), 2006.

Hirabayashi, Y., S. Kanae, S. Emori, T. Oki, and M. Kimoto, Global projections of changing risks of floods and droughts in a changing climate, Hydrological Sciences Journal, 53(4), 754-772, 2008.

Hodges, R. P., Underwater acoustics: Analysis, Design and Performance of Sonar, John Wiley \& Sons, 2011.

Hoitink, A., Z. Wang, B. Vermeulen, Y. Huismans, and K. Kästner, Tidal controls on river delta morphology, Nature Geoscience, 10(9), 637, 2017.

Hoitink, A. J. F., Tidally-induced clouds of suspended sediment connected to shallow-water coral reefs, Marine Geology, 208(1), 13-31, 2004.

Hoitink, A. J. F., and D. A. Jay, Tidal river dynamics: Implications for deltas, Rev. Geophys., 54(1), 240-272, 2016.

Hoitink, A. J. F., F. A. Buschman, and B. Vermeulen, Continuous measurements of discharge from a horizontal acoustic Doppler current profiler in a tidal river, Water Resources Research, 45(W10420), 2009.

Holdaway, G. P., P. D. Thorne, D. Flatt, S. E. Jones, and D. Prandle, Comparison between ADCP and transmissometer measurements of suspended sediment concentration, Continental Shelf Research, 19(3), 421-441, 1999.

Hood, W. G., Delta distributary dynamics in the Skagit River Delta (Washington, USA): extending, testing, and applying avulsion theory in a tidal system, Geomorphology, 123(1), 154-164, 2010.

Hooijer, A., S. Page, J. Jauhiainen, W. A. Lee, X. X. $\mathrm{Lu}, \mathrm{A}$. Idris, and G. Anshari, Subsidence and carbon loss in drained tropical peatlands, Biogeosciences, 9(3), 1053-1071, 2012.

Horrevoets, A. C., H. H. G. Savenije, J. N. Schuurman, and S. Graas, The influence of river discharge on tidal damping in alluvial estuaries, Journal of Hydrology, 294(4), 213-228, 2004.
Hunger, M., and P. Döll, Value of river discharge data for global-scale hydrological modeling, $\mathrm{Hy}$ drology and Earth System Sciences Discussions, 4(6), 4125-4173, 2007.

Hydrograaf, Westkust Borneo, Blad III, Pontianak tot Eiland Maya, 1882, 1907.

Ikeda, S., M. Yamasaka, and M. Chiyoda, Bed topography and sorting in bends, Journal of Hydraulic Engineering, 113(2), 190-204, 1987.

Ippen, A. T., Estuary and Coastline Hydrodynamics, McGraw-Hill Book Co., 1966.

Jackson, P. R., K. K. Johnson, and J. J. Duncker, Comparison of Index Velocity Measurements Made with a Horizontal Acoustic Doppler Current Profiler and a Three-path Acoustic Velocity Meter for Computation of Discharge in the Chicago Sanitary and Ship Canal Near Lemont, Illinois, U. S. Department of the Interior, U. S. Geological Survey, Reston, Virginia, U. S. A., 2012.

Jarrett, J. T., Tidal prism-inlet area relationships., Tech. rep., DTIC Document, 1976.

Jay, D. A., Green's law revisited: Tidal long-wave propagation in channels with strong topography, Journal of Geophysical Research: Oceans (1978-2012), 96(C11), 20,585-20,598, 1991.

Jay, D. A., and E. P. Flinchem, Interaction of fluctuating river flow with a barotropic tide: A. demonstration of wavelet tidal analysis methods, Journal of Geophysical Research: Oceans, 102(C3), 5705-5720, 1997.

Jay, D. A., and T. Kukulka, Revising the paradigm of tidal analysis-the uses of non-stationary data, Ocean Dynamics, 53(3), 110-125, 2003.

Jay, D. A., K. Leffler, H. L. Diefenderfer, and A. B. Borde, Tidal-fluvial and estuarine processes in the lower Columbia River: I. Along-channel water level variations, Pacific Ocean to Bonneville Dam, Estuaries and Coasts, 38(2), 415-433, 2015.

Jay, D. A., A. B. Borde, and H. L. Diefenderfer, TidalFluvial and Estuarine Processes in the Lower Columbia River: II. Water Level Models, Floodplain Wetland Inundation, and System Zones, Estuaries and Coasts, 39(5), 1299-1324, 2016.

Jerolmack, D. J., Conceptual framework for assessing the response of delta channel networks to Holocene sea level rise, Quaternary Science Reviews, 28(17), 1786-1800, 2009.

Jones, B. E., A Method of Correcting River Discharge for a Changing Stage, Contributions 
to the hydrology of the United States, United States Government Printing Office, 1916.

Jonkman, S. N., M. M. Hillen, R. J. Nicholls, W. Kanning, and M. van Ledden, Costs of adapting coastal defences to sea-level rise-new estimates and their implications, Journal of Coastal Research, 29(5), 1212-1226, 2013.

Kacimov, A. R., Note on a paper by Sinha and Odgaard "Application of conformal mapping to diverging open channel flow", Journal of Engineering Mathematics, 37(4), 397-400, 2000.

Kalkwijk, J. P. T., and R. Booij, Adaptation of secondary flow in nearly-horizontal flow, Journal of Hydraulic Research, 24(1), 19-37, 1986.

Kästner, K., and A. J. F. Hoitink, Flow and sediment division at two asymmetric bifurcations of a tidally influenced river delta: implications for channel stability, Submitted to Journal of Geophysical Research: Earth Surface, 2019a.

Kästner, K., and A. J. F. Hoitink, Separating the sensed from the unsensed fraction when inferring suspended sediment concentrations from mono-frequency acoustic backscatter, Submitted to Water Resources Research, 2019b.

Kästner, K., and A. J. F. Hoitink, The effect of the inlet width of lateral bifurcation branches on the division of sediment, Submitted to Journal of Fluid Mechanics, 2019c.

Kästner, K., and A. J. F. Hoitink, Effects of a sloping bed on the propagation of tides up-river, Submitted to Journal of Fluid Mechanics, 2019d.

Kästner, K., A. J. F. Hoitink, B. Vermeulen, T. J. Geertsema, and N. S. Ningsih, Distributary channels in the fluvial to tidal transition zone, Journal of Geophysical Research: Earth Surface, 3(122), 696-710, 2016JF004075, 2017.

Kästner, K., A. J. F. Hoitink, P. J. J. F. Torfs, B. Vermeulen, N. S. Ningsih, and M. Pramulya, Prerequisites for accurate monitoring of river discharge based on fixed-location velocity measurements, Water Resources Research, 54(2), 1058-1076, 2018.

Kawai, S., S. Egashra, and K. Ashida, Variation of Sediment Discharge over the Side Weir and Bed Variation in Straight Open Channel, Doboku Gakkai Ronbunshu, 1993(473), 7-15, 1993.

Kawanisi, K., M. Razaz, A. Kaneko, and S. Watanabe, Long-term measurement of stream flow and salinity in a tidal river by the use of the fluvial acoustic tomography system, Journal of $\mathrm{Hy}$ drology, 380(1), 74-81, 2010.
Kerssens, P. J. M., and A. Van Urk, Experimental studies on sedimentation due to water withdrawal, Journal of Hydraulic Engineering, 112(7), 641-656, 1986.

Kim, Y. H., and G. Voulgaris, Estimation of suspended sediment concentration in estuarine environments using acoustic backscatter from an ADCP, in Proceedings of Coastal Sediments, vol. 3, Citeseer, 2003.

Kimura, N., W.-C. Liu, C. H. Wu, A. J. Bechle, W.-B. Chen, and W.-C. Huang, Flow measurement with multi-instrumentation in a tidal-affected river, Water and Environment Journal, 25(4), 563572, 2011.

Kleinhans, M. G., H. R. A. Jagers, E. Mosselman, and C. J. Sloff, Bifurcation dynamics and avulsion duration in meandering rivers by onedimensional and three-dimensional models, $\mathrm{Wa}$ ter Resources Research, 44(8), 2008.

Kleinhans, M. G., R. I. Ferguson, S. N. Lane, and R. J. Hardy, Splitting rivers at their seams: bifurcations and avulsion, Earth Surface Processes and Landforms, 38(1), 47-61, 2013.

Kondolf, G., Z. Rubin, and J. Minear, Dams on the Mekong: cumulative sediment starvation, Water Resources Research, 50(6), 5158-5169, 2014.

Kostaschuk, R., P. Villard, and J. Best, Measuring velocity and shear stress over dunes with acoustic Doppler profiler, Journal of Hydraulic Engineering, 130(9), 932-936, 2004.

Ksiazek, L., and D. G. Meijer, Changes of Sediment Distribution in a Channel Bifurcation-3D Modeling, in Experimental Methods in Hydraulic Research, pp. 175-187, Springer, 2011.

Kukulka, T., and D. A. Jay, Impacts of Columbia River discharge on salmonid habitat: 2 . Changes in shallow-water habitat, Journal of Geophysical Research: Oceans, 108(C9), 2003a.

Kukulka, T., and D. A. Jay, Impacts of Columbia River discharge on salmonid habitat: 1. A. nonstationary fluvial tide model, Journal of Geophysical Research: Oceans (1978-2012), 108(C9), 2003b.

Kumagai, T., H. Kanamori, and T. Yasunari, Deforestation-induced reduction in rainfall, Hydrological Processes, 27(25), 3811-3814, 2013a.

Kumagai, T., H. Kanamori, and T. Yasunari, Deforestation-induced reduction in rainfall, Hydrological Processes, 27(25), 3811-3814, 
2013b.

Kvale, E. P., The origin of neap-spring tidal cycles, Marine Geology, 235(1-4), 5-18, 2006.

Lamb, H., Hydrodynamics, Cambridge University Press, 1932.

Lamb, M. P., J. A. Nittrouer, D. Mohrig, and J. Shaw, Backwater and river plume controls on scour upstream of river mouths: Implications for fluvio-deltaic morphodynamics, Journal of Geophysical Research: Earth Surface (2003-2012), 117(F1), 2012.

Latosinski, F. G., R. N. Szupiany, C. M. García, M. Guerrero, and M. L. Amsler, Estimation of concentration and load of suspended bed sediment in a large river by means of acoustic Doppler technology, Journal of Hydraulic Engineering, 140(7), 04014,023, 2014.

Le, T. B., A. Crosato, E. Mosselman, and W. S. J. Uijttewaal, On the stability of river bifurcations created by longitudinal training walls. Numerical investigation, Advances in Water Resources, 113, 112-125, 2018.

Le Coz, J., G. Pierrefeu, and A. Paquier, Evaluation of river discharges monitored by a fixed sidelooking Doppler profiler, Water Resources Research, 44(4), W00D09, 2008.

LeBlond, P. H., On tidal propagation in shallow rivers, Journal of Geophysical Research: Oceans, 83(C9), 4717-4721, 1978.

LeBlond, P. H., Forced fortnightly tides in shallow rivers, Atmosphere-Ocean, 17(3), 253-264, 1979.

Lebreton, L. C. M., J. Van der Zwet, J.-W. Damsteeg, B. Slat, A. Andrady, and J. Reisser, River plastic emissions to the world's oceans, Nature Communications, 8, 15,611, 2017.

Lee, T. H., and D. M. Hanes, Direct inversion method to measure the concentration profile of suspended particles using backscattered sound, Journal of Geophysical Research: Oceans, 100(C2), 2649-2657, 1995.

Leeder, M. R., and P. H. Bridges, Flow separation in meander bends, Nature, 253(5490), 338, 1975.

Leonardi, N., A. Canestrelli, T. Sun, and S. Fagherazzi, Effect of tides on mouth bar morphology and hydrodynamics, Journal of Geophysical Research: Oceans, 118(9), 4169-4183, 2013.

Leopold, L. B., and T. Maddock, The hydraulic geometry of stream channels and some physiographic implications, vol. 252, US Government
Printing Office, 1953.

Leopold, L. B., and T. Maddock Jr, The hydraulic geometry of stream channels and some physiographic implications, 252, United States Government Printing office, Washington, 1953.

Levesque, V. A., and K. A. Oberg, Computing discharge using the index velocity method, no. A23 in Techniques and Methods 3, 148 pp., U. S. Geological Survery, Reston, Virginia, U. S. A., 2012.

Li, C., and A. Valle-Levinson, A two-dimensional analytic tidal model for a narrow estuary of arbitrary lateral depth variation: The intratidal motion, Journal of Geophysical Research: Oceans, 104(C10), 23,525-23,543, 1999.

Li, C., M. J. Czapiga, E. C. Eke, E. Viparelli, and G. Parker, Variable Shields number model for river bankfull geometry: bankfull shear velocity is viscosity-dependent but grain sizeindependent, Journal of Hydraulic Research, 53(1), 36-48, 2015.

Lighthill, J., Waves in Fluids, Cambridge University Press, 2001.

Lorentz, H. A., Verslag Staatscommissie Zuiderzee 1918-1926, Algemene Landsdrukkerij,'sGravenhage, pp. 1-345, 1926.

Luke, S. H., H. Barclay, K. Bidin, V. K. Chey, R. M. Ewers, W. A. Foster, A. Nainar, M. Pfeifer, G. Reynolds, E. C. Turner, et al., The effects of catchment and riparian forest quality on stream environmental conditions across a tropical rainforest and oil palm landscape in Malaysian Borneo, Ecohydrology, 10(4), e1827, 2017.

Lynch, J. F., T. F. Gross, B. H. Brumley, and R. A. Filyo, Sediment concentration profiling in HEBBLE using a 1-MHz acoustic backscatter system, Marine Geology, 99(3-4), 361-385, 1991.

Maisels, C. K., Early civilizations of the Old World: the formative histories of Egypt, the Levant, Mesopotamia, India and China, Routledge, 2003.

Makaske, B., Anastomosing rivers: a review of their classification, origin and sedimentary products, Earth-Science Reviews, 53(3), 149196, 2001.

Malhi, Y., J. T. Roberts, R. A. Betts, T. J. Killeen, W. Li, and C. A. Nobre, Climate change, deforestation, and the fate of the amazon, Science, 319(5860), 169-172, 2008.

Marani, M., S. Lanzoni, D. Zandolin, G. Semi- 
nara, and A. Rinaldo, Tidal meanders, Water Resources Research, 38(11), 2002.

Marciano, R., Z. B. Wang, A. Hibma, H. J. De Vriend, and A. Defina, Modeling of channel patterns in short tidal basins, Journal of Geophysical Research: Earth Surface, 110(F1), 2005.

Mason, J., and D. Mohrig, Using time-lapse lidar to quantify river bend evolution on the meandering coastal Trinity River, Texas, USA, Journal of Geophysical Research: Earth Surface, 123(5), 1133-1144, 2018.

Matte, P., Y. Secretan, and J. Morin, Temporal and spatial variability of tidal-fluvial dynamics in the St. Lawrence fluvial estuary: An application of nonstationary tidal harmonic analysis, Journal of Geophysical Research: Oceans, 119(9), 5724-5744, 2014.

Matthes, G. H., Diversion of sediment at branching channels, Eos, Transactions American Geophysical Union, 14(1), 506-509, 1933.

McLaren, P., An interpretation of trends in grain size measures, Journal of Sedimentary Research, 51(2), 611-624, 1981.

McLean, S. R., Depth-integrated suspended-load calculations, Journal of Hydraulic Engineering, 117(11), 1440-1458, 1991.

McMillan, H., J. Freer, F. Pappenberger, T. Krueger, and M. Clark, Impacts of uncertain river flow data on rainfall-runoff model calibration and discharge predictions, Hydrological Processes, 24(10), 1270-1284, 2010.

McMillan, H., J. Seibert, A. Petersen-Overleir, M. Lang, P. White, T. Snelder, K. Rutherford, T. Krueger, R. Mason, and J. Kiang, How uncertainty analysis of streamflow data can reduce costs and promote robust decisions in water management applications, Water Resources Research, 53(7), 5220-5228, 2017.

Meade, R. H., River-Sediment Inputs to Major Deltas, in Sea-level Rise and Coastal Subsidence, pp. 63-85, Springer, 1996.

Meckel, T. A., U. S. Ten Brink, and S. J. Williams, Sediment compaction rates and subsidence in deltaic plains: numerical constraints and stratigraphic influences, Basin Research, 19(1), 1931, 2007.

Meijer, D. G., and L. Ksiazek, Sediment distribution in a channel bifurcation, Fluent simulation, Delft Hydraulics, Delft, The Netherlands, Reports Q1941, 1994.
Mellen, R. H., P. M. Scheifele, and D. G. Browning, Global model for sound absorption in sea water, Tech. rep., DTIC Document, 1987.

Merckelbach, L. M., and H. Ridderinkhof, Estimating suspended sediment concentration using backscatterance from an acoustic Doppler profiling current meter at a site with strong tidal currents, Ocean Dynamics, 56(3-4), 153-168, 2006.

Meteorological Service Singapore, Annual Climatological Report Singapore, 2014, 2014.

Meybeck, M., and A. Ragu, River discharges to the oceans: an assessment of suspended solids, major ions and nutrients, UNEP, 1995.

Michelazzo, G., H. Oumeraci, and E. Paris, Laboratory study on 3D flow structures induced by zero-height side weir and implications for 1D modeling, Journal of Hydraulic Engineering, 141(10), 04015,023, 2015.

Michelazzo, G., L. Minatti, E. Paris, and L. Solari, Side Weir Flow on a Movable Bed, Journal of Hydraulic Engineering, 142(6), 04016,007, 2016.

Michelazzo, G., H. Oumeraci, and E. Paris, New hypothesis for the final equilibrium stage of a river levee breach due to overflow, Water Resources Research, 2018.

Mikhailov, V. N., Hydrology and formation of river mouth bars, Problems of the Humid Tropical Zone Deltas, 1, 59-64, 1966.

Mikhailov, V. N., Hydrologic-morphometric characteristics of delta branches, Stud. Rep. Hydrol, 9, 146-158, 1970.

Milliman, J. D., and K. L. Farnsworth, River discharge to the coastal ocean: a global synthesis, Cambridge University Press, 2013.

Milliman, J. D., K. L. Farnsworth, and C. S. Albertin, Flux and fate of fluvial sediments leaving large islands in the East Indies, Journal of Sea Research, 41(1-2), 97-107, 1999.

Milly, P. C. D., K. A. Dunne, and A. V. Vecchia, Global pattern of trends in streamflow and water availability in a changing climate, Nature, 438(7066), 347, 2005.

Miori, S., R. Repetto, and M. Tubino, A onedimensional model of bifurcations in gravel bed channels with erodible banks, Water Resources Research, 42(11), 2006.

Modi, P. N., M. M. Dandekar, and P. D. Ariel, Conformal mapping for channel junction flow, Journal of the Hydraulics Division, 107(12), 1713-1733, 
1981.

Moftakhari, H. R., D. A. Jay, and S. A. Talke, Estimating river discharge using multiple-tide gauges distributed along a channel, Journal of Geophysical Research: Oceans, 121(4), 20782097, 2016.

Moore, S. A., J. Le Coz, D. Hurther, and A. Paquier, On the application of horizontal ADCPs to suspended sediment transport surveys in rivers, Continental Shelf Research, 46, 50-63, 2012.

Mopelwa, G., and J. Blignaut, The Okavango Delta: the value of tourism, South African Journal of Economic and Management Sciences, 9(1), 113-127, 2014.

Morris, J. T., P. V. Sundareshwar, C. T. Nietch, B. Kjerfve, and D. R. Cahoon, Responses of coastal wetlands to rising sea level, Ecology, 83(10), 2869-2877, 2002.

Moss, S. J., and M. E. J. Wilson, Biogeographic implications of the Tertiary palaeogeographic evolution of Sulawesi and Borneo, Biogeography and Geological Evolution of SE Asia, pp. 133163, 1998.

Mosselman, E., Theoretical bifurcation stability for rivers with adjusting width, in RCEM2017 Back tO Italy, 2017.

Murdiyarso, D., J. Purbopuspito, J. B. Kauffman, M. W. Warren, S. D. Sasmito, D. C. Donato, S. Manuri, H. Krisnawati, S. Taberima, and S. Kurnianto, The potential of Indonesian mangrove forests for global climate change mitigation, Nature Climate Change, 5(12), 1089, 2015.

Muste, M., and A. J. F. Hoitink, Measuring Flood Discharge, in Oxford Research Encyclopedia of Natural Hazard Science, Interactive Factory, Boston, Massachusetts, U. S. A., 2017.

Myrick, R. M., and L. B. Leopold, Hydraulic geometry of a small tidal estuary, Geological Survey Professional Paper, vol. 422 B, United States Government Printing Office, 1963.

Nardin, W., and S. Fagherazzi, The effect of wind waves on the development of river mouth bars, Geophysical Research Letters, 39(12), 2012.

Neary, V. S., F. Sotiropoulos, and A. J. Odgaard, Three-dimensional numerical model of lateralintake inflows, Journal of Hydraulic Engineering, 125(2), 126-140, 1999.

Nguyen, A. D., H. H. G. Savenije, D. N. Pham, and D. T. Tang, Using salt intrusion measurements to determine the freshwater discharge distribu- tion over the branches of a multi-channel estuary: The Mekong Delta case, Estuarine, Coastal and Shelf Science, 77(3), 433-445, 2008.

Nicholls, R. J., and A. Cazenave, Sea-level rise and its impact on coastal zones, Science, 328(5985), 1517-1520, 2010.

Nienhuis, J. H., A. D. Ashton, and L. Giosan, What makes a delta wave-dominated?, Geology, 43(6), 511-514, 2015.

Nienhuis, J. H., A. J. F. Hoitink, and T. E. Törnqvist, Future change to tide-influenced deltas, Geophysical Research Letters, 45(8), 3499-3507, 2018.

Nihei, Y., and A. Kimizu, A new monitoring system for river discharge with horizontal acoustic Doppler current profiler measurements and river flow simulation, Water Resources Research, 44(4), 2008.

Noe, G. B., and C. R. Hupp, Retention of riverine sediment and nutrient loads by coastal plain floodplains, Ecosystems, 12(5), 728-746, 2009.

Nordin, C. F., and B. S. Queen, Particle size distributions of bed sediments along the thalweg of the Mississippi River, Cairo, Illinois, to Head of Passes, September 1989, Tech. rep., DTIC Document, 1992.

Oki, T., and S. Kanae, Global hydrological cycles and world water resources, Science, 313(5790), 1068-1072, 2006.

Olariu, C., and J. P. Bhattacharya, Terminal distributary channels and delta front architecture of river-dominated delta systems, Journal of Sedimentary Research, 76(2), 212-233, 2006.

Önen, F., and H. Agaccioglu, Live bed scour at a side-weir intersection located on an alluvial channel, Irrigation and Drainage, 62(4), 488500, 2013.

Orton, G. J., and H. G. Reading, Variability of deltaic processes in terms of sediment supply, with particular emphasis on grain size, Sedimentology, 40(3), 475-512, 1993.

Ottevanger, W., K. Blanckaert, and W. S. J. Uijttewaal, Processes governing the flow redistribution in sharp river bends, Geomorphology, 163, 45-55, 2012.

Paris, E., L. Solari, and G. Bechi, Applicability of the De Marchi hypothesis for side weir flow in the case of movable beds, Journal of Hydraulic Engineering, 138(7), 653-656, 2012.

Parker, B., The relative importance of the various 
nonlinear mechanisms in a wide range of tidal interactions, in Tidal Hydrodynamics, pp. 125152, John Wiley \& Sons, New York, 1991.

Parker, B. B., Frictional effects on the tidal dynamics of a shallow estuary, Ph.D. thesis, Johns Hopkins University, Baltimore, Maryland, U. S. A, 1984.

Parker, B. B., Tidal Analysis and Prediction, NOAA Special Publication, NOAA, Maryland, Silver Spring, 2007.

Parker, B. B., and L. C. Huff, Modern under-keel clearance management, International Hydrographic Review, 75(2), 143-166, 1998.

Parker, G., Self-formed straight rivers with equilibrium banks and mobile bed. Part 2. The gravel river, Journal of Fluid Mechanics, 89(1), 127146, 1978.

Parker, G., T. Muto, Y. Akamatsu, W. E. Dietrich, and J. Wesley Lauer, Unravelling the conundrum of river response to rising sea-level from laboratory to field Part II: The Fly-Strickland River system, Papua New Guinea, Sedimentology, 55(6), 1657-1686, 2008.

Passalacqua, P., S. Lanzoni, C. Paola, and A. Rinaldo, Geomorphic signatures of deltaic processes and vegetation: The GangesBrahmaputra-Jamuna case study, Journal of Geophysical Research: Earth Surface, 118(3), 1838-1849, 2013.

Pawlowicz, R., B. Beardsley, and S. Lentz, Classical tidal harmonic analysis including error estimates in MATLAB using T TIDE, Computers \& Geosciences, 28(8), 929-93̄7, 2002.

Pelling, H. E., J. A. M. Green, and S. L. Ward, Modelling tides and sea-level rise: To flood or not to flood, Ocean Modelling, 63, 21-29, 2013.

Peters, H. J. F., Developments in global seatrade and container shipping markets: their effects on the port industry and private sector involvement, International Journal of Maritime Economics, 3(1), 3-26, 2001.

Phan, T. N. M., J. C. Wells, Y. Uchiyama, S. Ishii, J. S. Bonner, M. S. Islam, and W. D. Kirkey, Principal Component Analysis on Horizontal Acoustic Doppler Current Profilers Measurement in a Tidal River, Journal of JSCE B1 (Hydraulics), 70(4), I_73-I_78, 2014.

Phan, T. N. M., J. C. Wells, Y. Susuki, L. V. Nguyen, J. S. Bonner, M. S. Islam, and W. D. Kirkey, Koopman modes in a near-bank region of a tidal river, Journal of JSCE B1 (Hydraulics), 71(4), I_205-

\section{I_210, 2015.}

Phillips, J. D., Multiple modes of adjustment in unstable river channel cross-sections, Journal of Hydrology, 123(1-2), 39-49, 1991.

Pillsbury, G. B., Tidal Hydraulics, 34, United States Government Printing Office, 1940.

Ponce, V. M., and D. B. Simons, Shallow wave propagation in open channel flow, Journal of the Hydraulics Division, 103(American Society of Civil Engineers 13392 Proceeding), 1977.

Prandle, D., and A. Lane, Sensitivity of estuaries to sea level rise: Vulnerability indices, Estuarine, Coastal and Shelf Science, 160, 60-68, 2015.

Pritchard, D. W., What is an estuary: physical viewpoint, in Estuaries, vol. 83, edited by G. H. Lauff, pp. 3-5, American Association for the Advancement of Science, 1967.

Pugh, D. T., Tides, Surges and Mean Sea-Level: A Handbook for Engineers and Scientists, John Wiley, Chichester, UK, 1987.

Ramamurthy, A., J. Qu, and D. Vo, Numerical and experimental study of dividing openchannel flows, Journal of Hydraulic Engineering, 133(10), 1135-1144, 2007.

Rantz, S. E., Measurement and Computation of Streamflow: Volume 2 Computation of Discharge, no. 2175 in Geological Survey WaterSupply Paper, U. S. Geological Survey, 1982.

Raudkivi, A. J., Sedimentation: Exclusion and Removal of Sediment from Diverted Water, vol. 6, CRC Press, 1993.

Ray, R., G. Egbert, and S. Erofeeva, Tide predictions in shelf and coastal waters: Status and prospects, in Coastal Altimetry, pp. 191-216, Springer, 2011.

Ray, R. D., and D. E. Cartwright, Times of peak astronomical tides, Geophysical Journal International, 168(3), 999-1004, 2007.

Redolfi, M., G. Zolezzi, and M. Tubino, Free instability of channel bifurcations and morphodynamic influence, Journal of Fluid Mechanics, 799, 476-504, 2016.

Reef, K. R. G., G. Lipari, P. C. Roos, and S. J. M. H. Hulscher, Time-varying storm surges on lorentz's wadden sea networks, Ocean Dynamics, 68(8), 1051-1065, 2018.

Restrepo, J. D., A. Kettner, and J. P. Syvitski, Recent deforestation causes rapid increase in river sediment load in the Colombian Andes, Anthro- 
pocene, 10, 13-28, 2015.

Riad, K., Analytical and experimental study of bed load distribution at alluvial diversions, Ph.D. thesis, TU Delft, Delft University of Technology, 1961.

Richards, K., Rivers: Form and Processes in Alluvial Channels, Methuen, 1982.

Rijke, J., S. van Herk, C. Zevenbergen, and R. Ashley, Room for the River: delivering integrated river basin management in the Netherlands, International journal of river basin management, 10(4), 369-382, 2012.

Roberts, H. H., R. D. Adams, and R. H. W. Cunningham, Evolution of sand-dominant subaerial phase, Atchafalaya Delta, Louisiana, AAPG Bulletin, 64(2), 264-279, 1980.

Rodríguez-Iturbe, I., and A. Rinaldo, Fractal River Basins: Chance and Self-Organization, Cambridge University Press, 2001.

Rodríguez-Iturbe, I., and J. B. Valdes, The geomorphologic structure of hydrologic response, Water Resources Research, 15(6), 1409-1420, 1979.

Rönnbäck, P., The ecological basis for economic value of seafood production supported by mangrove ecosystems, Ecological Economics, 29(2), 235-252, 1999.

Rosier, B., Interaction of side weir overflow with bed-load transport and bed morphology in a channel, Ecole polytechnique federale de Lausanne, 2007.

Rosier, B., J.-L. Boillat, and A. J. Schleiss, Prediction of interaction between a side overflow and bedload transport in a channel with semi-empirical approaches, Canadian Journal of Civil Engineering, 36(11), 1755-1763, 2009.

Ross, A. C., R. G. Najjar, M. Li, M. E. Mann, S. E. Ford, and B. Katz, Sea-level rise and other influences on decadal-scale salinity variability in a coastal plain estuary, Estuarine, Coastal and Shelf Science, 157, 79-92, 2015.

Rowland, J. C., and W. E. Dietrich, The evolution of a tie channel, River, Coastal and Estuarine Morphodynamics: RCEM, 1, 725-736, 2005.

Rozovskii, I. L., Flow of water in bends of open channels, Academy of Sciences of the Ukrainian SSR, 1957.

Sahin, C., R. Verney, A. Sheremet, and G. Voulgaris, Acoustic backscatter by suspended cohesive sediments: Field observations, Seine Estu- ary, France, Continental Shelf Research, 134, 39-51, 2017.

Sakamoto, T., N. Van Nguyen, H. Ohno, N. Ishitsuka, and M. Yokozawa, Spatio-temporal distribution of rice phenology and cropping systems in the Mekong Delta with special reference to the seasonal water flow of the Mekong and Bassac rivers, Remote Sensing of Environment, 100(1), 1-16, 2006.

Salter, G., C. Paola, and V. R. Voller, Control of delta avulsion by downstream sediment sinks, Journal of Geophysical Research: Earth Surface, 123(1), 142-166, 2018.

Sassi, M. G., Discharge regimes, tides and morphometry in the Mahakam delta channel network, Ph.D. thesis, Wageningen University, Wageningen, The Netherlands, 2013.

Sassi, M. G., and A. J. F. Hoitink, River flow controls on tides and tide-mean water level profiles in a tidal freshwater river, Journal of Geophysical Research: Oceans, 118(9), 4139-4151, 2013.

Sassi, M. G., A. J. F. Hoitink, B. Brye, and E. Deleersnijder, Downstream hydraulic geometry of a tidally influenced river delta, Journal of Geophysical Research: Earth Surface, 117(F4), 2012.

Sassi, M. G., A. J. F. Hoitink, B. Brye, B. Vermeulen, and E. Deleersnijder, Tidal impact on the division of river discharge over distributary channels in the Mahakam Delta, Ocean Dynamics, 61(12), 2211-2228, 2011a.

Sassi, M. G., A. J. F. Hoitink, and B. Vermeulen, Discharge estimation from $\mathrm{H}$-ADCP measurements in a tidal river subject to sidewall effects and a mobile bed, Water Resources Research, 47(6), W06,504, 2011b.

Sassi, M. G., A. J. F. Hoitink, and B. Vermeulen, Impact of sound attenuation by suspended sediment on ADCP backscatter calibrations, Water Resources Research, 48(9), W09,520, 2012.

Sassi, M. G., A. J. F. Hoitink, B. Vermeulen, and H. Hidayat, Sediment discharge division at two tidally influenced river bifurcations, Water Resources Research, 49(4), 2119-2134, 2013.

Savenije, H. H. G., Predictive model for salt intrusion in estuaries, Journal of Hydrology, 148(14), 203-218, 1993.

Savenije, H. H. G., A simple analytical expression to describe tidal damping or amplification, Journal of Hydrology, 243(3), 205-215, 2001. 
Savenije, H. H. G., Salinity and Tides in Alluvial Estuaries, 2nd completely revised edition, salinityandtides.com, 2012a.

Savenije, H. H. G., Salinity and Tides in Alluvial Estuaries, 2nd edition, salinityandtides.com, 2012b.

Savenije, H. H. G., Prediction in ungauged estuaries: An integrated theory, Water Resources Research, 51(4), 2464-2476, 2015.

Savenije, H. H. G., and E. J. M. Veling, Relation between tidal damping and wave celerity in estuaries, Journal of Geophysical Research: Oceans, 110(C4), 2005.

Savenije, H. H. G., M. Toffolon, J. Haas, and E. J. M. Veling, Analytical description of tidal dynamics in convergent estuaries, Journal of Geophysical Research: Oceans, 113(C10), 2008.

Saviour, M. N., Environmental impact of soil and sand mining: a review, International Journal of Science, Environment and Technology, 1(3), 125-134, 2012.

Schaafsma, A. S., A. M. Lafort, and D. Guyomar, Development of an acoustic method and prototype instrumentation for size and concentration measurement of suspended sediment, in Elsevier Oceanography Series, vol. 62, pp. 168-175, Elsevier, 1997.

Schielen, R. M. J., P. Jesse, and L. Botwidt, On the use of flexible spillways to control the discharge ratio of the Rhine in the Netherlands: hydraulic and morphological observations, Netherlands Journal of Geosciences, 86(1), 77-88, 2007.

Schielen, R. M. J., H. Havinga, and M. Lemans, Dynamic control of the discharge distributions of the Rhine River in the Netherlands, in Proceedings of the International Conferference on Fluvial Hydraulics, River Flow, pp. 3-5, 2008.

Schönfeld, J. C., Propagation of tides and similar waves, 1951.

Schumm, S. A., Patterns of alluvial rivers, Annual Review of Earth and Planetary Sciences, 13, 5, 1985.

Scott, D. W., Multivariate density estimation: theory, practice, and visualization, John Wiley \& Sons, 2015.

Seminara, G., M. B. Pittaluga, and N. Tambroni, Morphodynamic equilibrium of tidal channels, in Environmental Fluid Mechanics-Memorial Volume in Honour of Prof. Gerhard Jirka, edited by W. Rodi and M. Uhlmann, pp. 153-174, CRC
Press, Karlsruhe, Germany, 2012.

Sexton, W. J., and M. Murday, The Morphology and Sediment Character of the Coastline of Nigeria: the Niger Delta, Journal of Coastal Research, pp. 959-977, 1994.

Shaw, J. B., and D. Mohrig, The importance of erosion in distributary channel network growth, Wax Lake Delta, Louisiana, USA, Geology, 42(1), 31-34, 2014.

Shaw, J. B., D. Mohrig, and R. W. Wagner, Flow patterns and morphology of a prograding river delta, Journal of Geophysical Research: Earth Surface, 121(2), 372-391, 2016.

Shettar, A. S., and K. K. Murthy, A numerical study of division of flow in open channels, Journal of Hydraulic Research, 34(5), 651-675, 1996.

Shiono, K., and D. W. Knight, Two-dimensional analytical solution for a compound channel, in Proc., 3rd Int. Symp. on refined flow modeling and turbulence measurements, pp. 503-510, Tokyo, Japan, 1989.

Sibinga, S., On the origin of the drainage system of Borneo, Geologie en Mijnbouw, 15, 121-136, 1953.

Simpson, M. R., and R. Bland, Methods for accurate estimation of net discharge in a tidal channel, IEEE Journal of Oceanic Engineering, 25(4), 437-445, 2000.

Singh, M., I. B. Singh, and G. Müller, Sediment characteristics and transportation dynamics of the Ganga River, Geomorphology, 86(1), 144175, 2007.

Sinha, S. K., and A. J. Odgaard, Applications of conformal mapping to diverging open channel flows, Journal of Engineering Mathematics, 30(3), 355-363, 1996.

Slingerland, R., and N. D. Smith, Necessary conditions for a meandering-river avulsion, Geology, 26(5), 435-438, 1998.

Slingerland, R., and N. D. Smith, River avulsions and their deposits, Annual Review of Earth and Planetary Sciences, 32, 257-285, 2004.

Sloff, C. J., Mixed alluvial and non-alluvial bed topographies: observations, modeling and implications, River Flow 2010, 2010.

Sloff, K., and E. Mosselman, Bifurcation modelling in a meandering gravel-sand bed river, Earth Surface Processes and Landforms, 37(14), 1556-1566, 2012. 
Sloff, K., A. Van Spijk, E. Stouthamer, and A. Sieben, Understanding and managing the morphology of branches incising into sand-clay deposits in the dutch rhine delta, International Journal of Sediment Research, 28(2), 127-138, 2013.

Smakhtin, V. U., Low Flow Hydrology: A Review, Journal of Hydrology, 240(3-4), 147-186, 2001.

Small, C., and R. J. Nicholls, A global analysis of human settlement in coastal zones, Journal of Coastal Research, pp. 584-599, 2003.

Smith, G. H. S., and R. I. Ferguson, The gravel-sand transition along river channels, Journal of Sedimentary Research, 65(2), 1995.

Smith, L. C., Satellite remote sensing of river inundation area, stage, and discharge: A. review, $\mathrm{Hy}$ drological Processes, 11(10), 1427-1439, 1997.

Snow, R. S., and R. L. Slingerland, Mathematical modeling of graded river profiles, The Journal of Geology, 1995(1), 15-33, 1987.

Snyder, P. K., The influence of tropical deforestation on the northern hemisphere climate by atmospheric teleconnections, Earth Interactions, 14(4), 1-34, 2010.

Song, T., and W. H. Graf, Non-uniform openchannel flow over a rough bed, Journal of Hydroscience and Hydraulic Engineering, 12(1), 1-25, 1994.

Souchay, J., S. Mathis, and T. Tokieda, Tides in Astronomy and Astrophysics, vol. 861, Springer, 2012.

Speer, P. E., and D. G. Aubrey, A study of non-linear tidal propagation in shallow inlet/estuarine systems Part II: Theory, Estuarine, Coastal and Shelf Science, 21(2), 207-224, 1985.

Sreebha, S., and D. Padmalal, Environmental impact assessment of sand mining from the small catchment rivers in the southwestern coast of India: A case study, Environmental Management, 47(1), 130-140, 2011.

Staub, J. R., and R. A. Gastaldo, Seasonal sediment transport and deposition in the Rajang River delta, Sarawak, East Malaysia, Sedimentary Geology, 133(3-4), 249-264, 2000.

Stecca, G., R. Measures, and D. M. Hicks, A framework for the analysis of noncohesive bank erosion algorithms in morphodynamic modeling, Water Resources Research, 53(8), 6663-6686, 2017.

Stevaux, J. C., and I. A. Souza, Floodplain con- struction in an anastomosed river, Quatern. Int., 114(1), 55-65, 2004.

Stouthamer, E., and H. J. A. Berendsen, Avulsion: the relative roles of autogenic and allogenic processes, Sedimentary Geology, 198(3-4), 309325, 2007.

Stouthamer, E., K. M. Cohen, and M. J. P. Gouw, Avulsion and its implications for fluvial-deltaic architecture: insights from the Holocene RhineMeuse delta, SEPM Special Publication, 97, 215-232, 2011.

Sutjipto, R. H., Sedimentology of the Melawi and Kentungau Basins, West Kalimantan, Indonesia, Ph.D. thesis, University of Wollongong, Wollongong, NSW, Australia, 1991.

Syvitski, J. P., S. Cohen, A. J. Kettner, and G. R. Brakenridge, How important and different are tropical rivers? - An overview, Geomorphology, 227, 5-17, 2014.

Syvitski, J. P. M., and Y. Saito, Morphodynamics of deltas under the influence of humans, Global and Planetary Change, 57(3-4), 261-282, 2007.

Syvitski, J. P. M., A. J. Kettner, A. Correggiari, and B. W. Nelson, Distributary channels and their impact on sediment dispersal, Marine Geology, $222,75-94,2005 a$.

Syvitski, J. P. M., A. J. Kettner, A. Correggiari, and B. W. Nelson, Distributary channels and their impact on sediment dispersal, Marine Geology, 222, 75-94, 2005b.

Syvitski, J. P. M., A. J. Kettner, I. Overeem, E. W. H. Hutton, M. T. Hannon, G. R. Brakenridge, J. Day, C. Vörösmarty, Y. Saito, L. Giosan, et al., Sinking deltas due to human activities, Nature Geoscience, 2(10), 681, 2009.

Syvitski, J. P. M., C. J. Vörösmarty, A. J. Kettner, and P. Green, Impact of humans on the flux of terrestrial sediment to the global coastal ocean, Science, 308(5720), 376-380, 2005.

Szupiany, R. N., M. L. Amsler, D. R. Parsons, and J. L. Best, Morphology, flow structure, and suspended bed sediment transport at two large braid-bar confluences, Water Resources Research, 45(5), 2009.

Takahashi, A., T. Kumagai, H. Kanamori, H. Fujinami, T. Hiyama, and M. Hara, Impact of Tropical Deforestation and Forest Degradation on Precipitation over Borneo Island, Journal of $\mathrm{Hy}$ drometeorology, 18(11), 2907-2922, 2017.

Talmon, A. M., Bed topography of river bends with 
suspended sediment transport, Ph.D. thesis, TU Delft, 1992.

Tarya, A., A. Hoitink, M. Van der Vegt, M. van Katwijk, B. Hoeksema, T. Bouma, L. Lamers, and M. Christianen, Exposure of coastal ecosystems to river plume spreading across a nearequatorial continental shelf, Continental Shelf Research, 153, 1-15, 2018.

Tattersall, G. R., A. J. Elliott, and N. M. Lynn, Suspended sediment concentrations in the Tamar estuary, Estuarine, Coastal and Shelf Science, 57(4), 679-688, 2003.

Taufik, M., Hydrological drought and wildfire in the humid tropics, Ph.D. thesis, Wageningen University, 2017.

Taufik, M., B. I. Setiawan, and H. A. J. van Lanen, Modification of a fire drought index for tropical wetland ecosystems by including water table depth, Agricultural and Forest Meteorology, 203, 1 - 10, 2015.

Taufik, M., P. J. J. F. Torfs, R. Uijlenhoet, P. D. Jones, D. Murdiyarso, and H. A. J. Van Lanen, Amplification of wildfire area burnt by hydrological drought in the humid tropics, Nature Climate Change, 7(6), 428, 2017.

Taufik, M., J. V. Dam, B. I. Setiawan, H. Wösten, P. D. Jones, and H. A. J. V. Lanen, Human contribution to increased drought severity in Southeast Asian peatland, Science of the Total Environment, under review, 2018a.

Taufik, M., B. I. Setiawan, and H. A. J. V. Lanen, Increased fire hazard in human-modified wetlands in Southeast Asia, AMBIO, under review, 2018b.

ten Brinke, W. B. M., De bodemsamenstelling van Waal en IJssel in de jaren 1966, 1976, 1984 en 1995, RIZA, Arnhem, 1997.

Ter Bruggen, G., De eocene fyllietformatie in Centraal-Borneo, Waltman, 1935.

Terra, G. M., W. J. van de Berg, and L. R. M. Maas, Experimental verification of Lorentz' linearization procedure for quadratic friction, Fluid $D y$ namics Research, 36(3), 175-188, 2005.

Thorne, P. D., and D. M. Hanes, A review of acoustic measurement of small-scale sediment processes, Continental Shelf Research, 22(4), 603632, 2002.

Thorne, P. D., and D. Hurther, An overview on the use of backscattered sound for measuring suspended particle size and concentration profiles in non-cohesive inorganic sediment trans- port studies, Continental Shelf Research, 73, 97-118, 2014.

Thorne, P. D., and R. Meral, Formulations for the scattering properties of suspended sandy sediments for use in the application of acoustics to sediment transport processes, Continental Shelf Research, 28(2), 309-317, 2008.

Tockner, K., and J. A. Stanford, Riverine flood plains: present state and future trends, Environmental Conservation, 29(3), 308-330, 2002.

Toffolon, M., G. Vignoli, and M. Tubino, Relevant parameters and finite amplitude effects in estuarine hydrodynamics, Journal of Geophysical Research: Oceans, 111(C10), 2006.

Topping, D. J., S. A. Wright, T. S. Melis, and D. M. Rubin, High-resolution measurements of suspended-sediment concentration and grain size in the Colorado River in Grand Canyon using a multi-frequency acoustic system, in Proceedings of the 10th International Symposium on River Sedimentation, vol. 3, 2007.

Torres, R., Channel geomorphology along the fluvial-tidal transition, Santee River, USA, GSA Bulletin, 129(11-12), 1681-1691, 2017.

Turnipseed, D. P., and V. B. Sauer, Discharge measurements at gaging stations, Tech. rep., US Geological Survey, 2010.

Van, C. P., O. Gourgue, M. Sassi, A. Hoitink, E. Deleersnijder, and S. Soares-Frazão, Modelling fine-grained sediment transport in the Mahakam land-sea continuum, Indonesia, Journal of Hydro-Environment Research, 13, 103-120, 2016.

van Bemmelen, R. W., The geology of Indonesia. 1, A. General geology of Indonesia and adjacent archipelagoes, US Government Printing Office, 1949.

van Denderen, R. P., R. M. J. Schielen, A. Blom, S. J. M. H. Hulscher, and M. G. Kleinhans, Morphodynamic assessment of side channel systems using a simple one-dimensional bifurcation model and a comparison with aerial images, Earth Surface Processes and Landforms, 43(6), 1169-1182, 2018.

van der Mark, C. F., and E. Mosselman, Effects of helical flow in one-dimensional modelling of sediment distribution at river bifurcations, Earth Surface Processes and Landforms, 38(5), 502511, 2013.

van Oudenhoven, A. P., A. J. Siahainenia, I. Sualia, F. H. Tonneijck, S. van der Ploeg, R. S. de Groot, 
R. Alkemade, and R. Leemans, Effects of different management regimes on mangrove ecosystem services in java, indonesia, Ocean \& Coastal Management, 116, 353-367, 2015.

van Rijn, L. C., Sediment transport, part III: bed forms and alluvial roughness, Journal of Hydraulic Engineering, 110(12), 1733-1754, 1984a.

van Rijn, L. C., Analytical and numerical analysis of tides and salinities in estuaries; part I: tidal wave propagation in convergent estuaries, Ocean Dynamics, 61(11), 1719-1741, 2011.

van Rijn, L. C. v., Sediment transport, part II: suspended load transport, Journal of Hydraulic Engineering, 110(11), 1613-1641, 1984b.

Varadan, V. V., Y. Ma, and V. K. Varadan, Theoretical analysis of the acoustic response of suspended sediment for HEBBLE, Marine Geology, 66(1), 267-276, 1985.

Vatankhah, A. R., and S. M. Easa, Direct integration of Manning-based gradually varied flow equation, in Proceedings of the Institution of Civil Engineers-Water Management, vol. 164, pp. 257-264, Thomas Telford Ltd, 2011.

Vellinga, N., A. Hoitink, M. van der Vegt, W. Zhang, and P. Hoekstra, Human impacts on tides overwhelm the effect of sea level rise on extreme water levels in the Rhine-Meuse delta, Coastal Engineering, 90, 40-50, 2014.

Venditti, J. G., M. Church, M. E. Attard, and D. Haught, Use of ADCPs for suspended sediment transport monitoring: An empirical approach, Water Resources Research, 52(4), 2715-2736, 2016.

Vermeulen, B., Rivers running deep, Ph.D. thesis, Wageningen University, Wageningen, The Netherlands, 2014.

Vermeulen, B., A. J. F. Hoitink, S. W. Berkum, and $\mathrm{H}$. Hidayat, Sharp bends associated with deep scours in a tropical river: The river Mahakam (East Kalimantan, Indonesia), Journal of Geophysical Research: Earth Surface, 119(7), 1441-1454, 2014a.

Vermeulen, B., M. G. Sassi, and A. J. F. Hoitink, Improved flow velocity estimates from movingboat ADCP measurements, Water Resources Research, 50(5), 4186-4196, 2014b.

Vermeulen, B., A. J. F. Hoitink, and R. J. Labeur, Flow structure caused by a local cross-sectional area increase and curvature in a sharp river bend, Journal of Geophysical Research: Earth
Surface, 120(9), 1771-1783, 2015.

Vermeulen, B., A. K. Huisman, A. J. F. Hoitink, and M. Pramulya, Migration of banks along the Kapuas River, West Kalimantan, in River Flow-Proceedings of the International Conference on Fluvial Hydraulics, RIVER FLOW 2016, pp. 1249-1253, 2016.

Voris, H. K., Maps of Pleistocene sea levels in Southeast Asia: shorelines, river systems and time durations, Journal of Biogeography, 27(5), 1153-1167, 2000.

Vörösmarty, C. J., C. Léveque, C. Revenga, R. Bos, C. Caudill, J. Chilton, E. M. Douglas, M. Meybeck, D. Prager, P. Balvanera, et al., Fresh water, Millennium Ecosystem Assessment, 1, 165-207, 2005.

Vougioukas, S., D. Papamichail, P. Georgiou, and D. Papadimos, River discharge monitoring using a vertically moving side-looking acoustic Doppler profiler, Computers and Electronics in Agriculture, 79(2), 137-141, 2011.

Wall, G. R., E. A. Nystrom, and S. Litten, Use of an ADCP to compute suspended-sediment discharge in the tidal Hudson River, New York, Tech. rep., U. S. Geological Survey, 2006.

Wang, S., J. Ni, G. Wang, D. Cheng, and O. Zhang, Hydrological processes of an anastomosing river system on the Zhujiang River Delta, China, J. Coastal. Res., Special issue 43: Tidal Dynamics and Environment, 124-133, 2004.

Wang, Z. B., and P. Ding, The branching channel network in the Yangtze estuary, Coastal Engineering Proceedings, 1(33), 69, 2012.

Wang, Z. B., M. De Vries, R. J. Fokkink, and A. Langerak, Stability of river bifurcations in 1D morphodynamic models, Journal of Hydraulic Research, 33(6), 739-750, 1995.

Ward, J. V., K. Tockner, and F. Schiemer, Biodiversity of floodplain river ecosystems: ecotones and connectivity, Regulated rivers: research \& management, 15(1), 125-139, 1999.

Ward, P. J., W. Beets, L. M. Bouwer, J. C. J. H. Aerts, and $\mathrm{H}$. Renssen, Sensitivity of river discharge to ENSO, Geophysical Research Letters, 37(12), 2010.

Wassmann, R., N. X. Hien, C. T. Hoanh, and T. P. Tuong, Sea level rise affecting the Vietnamese Mekong Delta: water elevation in the flood season and implications for rice production, Climatic Change, 66(1-2), 89-107, 2004. 
Watson, I., and C. W. Finkl Jr, State of the art in storm-surge protection: The Netherlands Delta Project, Journal of Coastal Research, pp. 739764, 1990.

Webster, P. J., and C. Hoyos, Prediction of monsoon rainfall and river discharge on 15-30-day time scales, Bulletin of the American Meteorological Society, 85(11), 1745-1765, 2004.

Wei, Z., G. Fang, R. D. Susanto, T. R. Adi, B. Fan, A. Setiawan, S. Li, Y. Wang, and X. Gao, Tidal elevation, current, and energy flux in the area between the South China Sea and Java Sea, Ocean Science, 12(2), 517-531, 2016.

Wells, J. T., Tide-dominated estuaries and tidal rivers, in Developments in Sedimentology, vol. 53, pp. 179-205, Elsevier, 1995.

Weng, Q., Land use change analysis in the Zhujiang Delta of China using satellite remote sensing, GIS and stochastic modelling, Journal of Environmental Management, 64(3), 273-284, 2002.

Wilkerson, G. V., and G. Parker, Physical basis for quasi-universal relationships describing bankfull hydraulic geometry of sand-bed rivers, Journal of Hydraulic Engineering, 137(7), 739-753, 2010.

Wilkinson, R. H., A method for evaluating statistical errors associated with logarithmic velocity profiles, Geo-marine letters, 3(1), 49-52, 1983.

Wilmer III, A., and G. B. Costa, Solving secondorder differential equations with variable coefficients, International Journal of Mathematical Education in Science and Technology, 39(2), 238-243, 2008.

Wilson, M. E. J., and S. J. Moss, Cenozoic palaeogeographic evolution of Sulawesi and Borneo, Palaeogeography, Palaeoclimatology, Palaeoecology, 145(4), 303-337, 1999.

Wing Easton, N., Voorloopige mededeelingen over de Geologie van het stroomgebied der Kapoeasrivier in de Westerafdeeling van Borneo, Tijdschr. Kon. Ned. Aard. Genootschap, 2de Serie, deel, 16, 245, 1899.

Wise, R., J. Butler, W. Suadnya, K. Puspadi, I. Suharto, and T. Skewes, How climate compatible are livelihood adaptation strategies and development programs in rural indonesia?, Climate Risk Management, 12, 100-114, 2016.

Withers, P. J. A., and H. P. Jarvie, Delivery and cycling of phosphorus in rivers: a review, Science of the Total Environment, 400(1-3), 379-395, 2008.
Witting, J. M., A note on Green's law, Journal of Geophysical Research: Oceans, 86(C3), 19951999, 1981.

Wolanski, E., N. N. Huan, N. H. Nhan, N. N. Thuy, et al., Fine-sediment dynamics in the Mekong River estuary, Vietnam, Estuarine, Coastal and Shelf Science, 43(5), 565-582, 1996.

Woodroffe, C. D., The impact of sea-level rise on mangrove shorelines, Progress in Physical Geography, 14(4), 483-520, 1990.

Woodworth, P. L., Differences between mean tide level and mean sea level, Journal of Geodesy, 91(1), 69-90, 2017.

Wright, L. D., River deltas, in Coastal sedimentary environments, pp. 5-68, Springer, 1978.

Wright, L. D., J. M. Coleman, and B. G. Thom, Processes of channel development in a high-tiderange environment: Cambridge Gulf-Ord River Delta, Western Australia, The Journal of Geology, 81(1), 15-41, 1973.

Wright, S., and G. Parker, Modeling downstream fining in sand-bed rivers. I: Formulation, Journal of Hydraulic Research, 43(6), 613-620, 2005.

Wright, S. A., D. J. Topping, and C. A. Williams, Discriminating silt-and-clay from suspended-sand in rivers using side-looking acoustic profilers, in Joint Federal Interagency Conference 2010: Hydrology and sedimentation for a changing future: existing and emerging issues, 2010.

Xiaoqing, Y., and X. Yang, Manual on sediment management and measurement, Secretariat of the World Meteorological Organization, 2003.

Yang, S. L., J. D. Milliman, P. Li, and K. Xu, 50,000 dams later: erosion of the Yangtze River and its delta, Global and Planetary Change, 75(1-2), 14-20, 2011.

Yang, S.-Q., W.-L. Xu, and G.-L. Yu, Velocity distribution in a gradually accelerating free surface flow, Advances in Water Resources, 29(12), 1969-1980, 2006.

Yule, C. M., L. Boyero, and R. Marchant, Effects of sediment pollution on food webs in a tropical river (Borneo, Indonesia), Marine and Freshwater Research, 61(2), 204-213, 2010.

Yusuf, A. A., and H. Francisco, Climate change vulnerability mapping for Southeast Asia, Tech. rep., Economy and Environment Program for Southeast Asia (EEPSEA), Singapore, SG, 2009.

Zhou, Z., G. Coco, I. Townend, M. Olabarrieta, M. Van Der Wegen, Z. Gong, A. D’alpaos, S. Gao, 
B. E. Jaffe, G. Gelfenbaum, et al., Is "morphodynamic equilibrium" an oxymoron?, EarthScience Reviews, 165, 257-267, 2017.

Zinger, J. A., B. L. Rhoads, J. L. Best, and K. K. Johnson, Flow structure and channel morphodynamics of meander bend chute cutoffs: A. case study of the Wabash River, USA, Journal of Geophysical Research: Earth Surface, 118(4), 2468-2487, 2013.

Zolezzi, G., W. Bertoldi, M. Tubino, G. H. S. Smith, J. L. Best, C. S. Bristow, and G. E. Petts, Morphological analysis and prediction of river bifurcations, Braided Rivers: Process, Deposits, Ecology and Management (Special Publication 36 of the IAS), 59, 233, 2009. 



\section{SENSE}

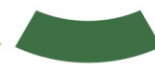

Netherlands Research School for the

Socio-Economic and Natural Sciences of the Environment

\section{I P L O M A}

For specialised PhD training

The Netherlands Research School for the Socio-Economic and Natural Sciences of the Environment

(SENSE) declares that

\section{Karl Kästner}

born on 6 June 1984 in Halle (Saale), Germany

has successfully fulfilled all requirements of the Educational Programme of SENSE.

Wageningen, 1 May 2019

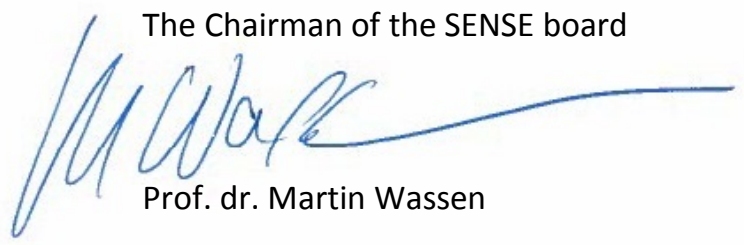

the SENSE Director of Education

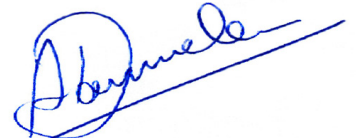

Dr. Ad van Dommelen

The SENSE Research School has been accredited by the Royal Netherlands Academy of Arts and Sciences (KNAW)

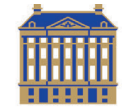

$\begin{array}{llllllllllllllllllllll}K & O & N & I & N & K & L & I & J & K & E & N & E & D & E & R & L & A & N & D & S & E\end{array}$

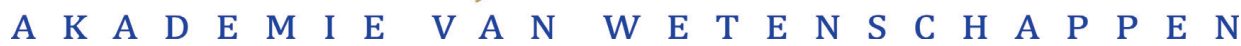




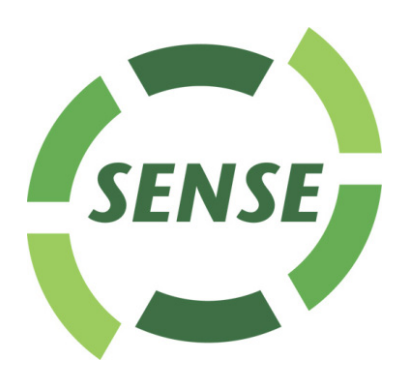

The SENSE Research School declares that Karl Kästner has successfully fulfilled all requirements of the Educational PhD Programme of SENSE with a work load of 45.4 EC, including the following activities:

\section{SENSE PhD Courses}

- Environmental research in context (2013)

- Research in context activity: "'Co-organizing short course on 'Monitoring flow, discharge, turbulence and suspended sediment from ADCPs' and co-producing workshop manual (August28 -August 30, 2015, Iquitos, Peru)" (2015)

- Bayesian statistics (2014)

- Generalized linear models (2015)

- SENSE writing week (2016)

- Career orientation (2017)

\section{Other PhD and Advanced MSc Courses}

o River flow and morphology, Wageningen University (2013)

- Hydrological processes in catchments, Wageningen University (2013)

- Delft 3D hydrodynamics, Deltares (2015)

- Delft 3D morphology, Deltares (2015)

- Scientific writing, Wageningen Graduate Schools (2016)

\section{Management and Didactic Skills Training}

o Supervising five BSc student with thesis entitled (2014-2016)

o Supervising three MSc students with thesis (2014-2017)

\section{Oral Presentations}

- Uncertainty of discharge monitoring with horizontal ADCPs. International Association for Hydro-Environment Engineering and Research (IAHR), 28 June -3 July 2015, The Hague, The Netherlands

- Do distributaries in a delta plain resemble an ideal estuary? Results from the Kapuas Delta, Indonesia. Netherlands Centre for River Studies, NCR Days, 01-03 February 2017, Wageningen, The Netherlands

- Distributary channels in the fluvial-tidal transition: or how ideal are real estuaries? The case of the Kapuas Delta, Indonesia. Physics of Estuaries and Coastal Seas (PECS), 0914 October 2016, The Hague, The Netherlands

- Monitoring flow and sediment transport at strongly asymmetric bifurcations of a large sand-bedded river, Netherlands Centre for River studies(NCR) days, 31 January-1 February 2019, Utrecht, The Netherlands 2019

SENSE Coordinator PhD Education

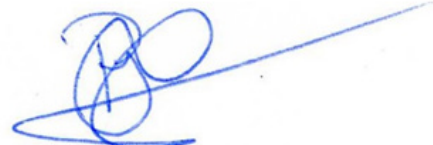

Dr. Peter Vermeulen 


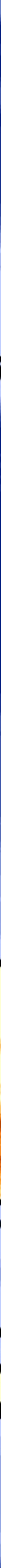




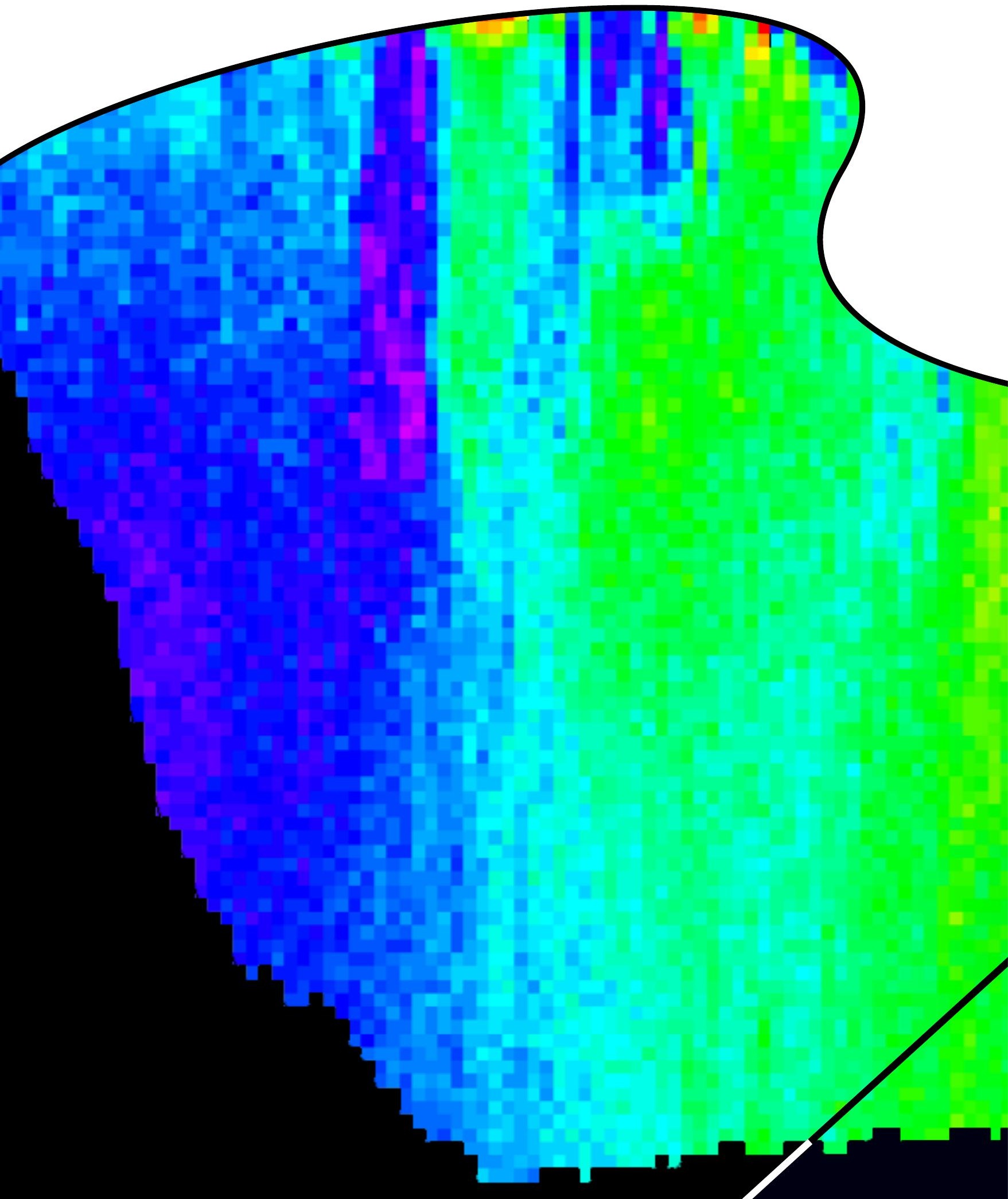

